\title{
Online usage inequalities
}

Citation for published version (APA):

Reinwand, D. A. (2016). Online usage inequalities: internet and Web-based health intervention use among people with different personal characteristics. [Doctoral Thesis, Maastricht University]. Datawyse / Universitaire Pers Maastricht. https://doi.org/10.26481/dis.20161221dr

\section{Document status and date:}

Published: 01/01/2016

DOI:

10.26481/dis.20161221dr

Document Version:

Publisher's PDF, also known as Version of record

\section{Please check the document version of this publication:}

- A submitted manuscript is the version of the article upon submission and before peer-review. There can be important differences between the submitted version and the official published version of record.

People interested in the research are advised to contact the author for the final version of the publication, or visit the DOI to the publisher's website.

- The final author version and the galley proof are versions of the publication after peer review.

- The final published version features the final layout of the paper including the volume, issue and page numbers.

Link to publication

\footnotetext{
General rights rights.

- You may freely distribute the URL identifying the publication in the public portal. please follow below link for the End User Agreement:

www.umlib.nl/taverne-license

Take down policy

If you believe that this document breaches copyright please contact us at:

repository@maastrichtuniversity.nl

providing details and we will investigate your claim.
}

Copyright and moral rights for the publications made accessible in the public portal are retained by the authors and/or other copyright owners and it is a condition of accessing publications that users recognise and abide by the legal requirements associated with these

- Users may download and print one copy of any publication from the public portal for the purpose of private study or research.

- You may not further distribute the material or use it for any profit-making activity or commercial gain

If the publication is distributed under the terms of Article $25 \mathrm{fa}$ of the Dutch Copyright Act, indicated by the "Taverne" license above, 


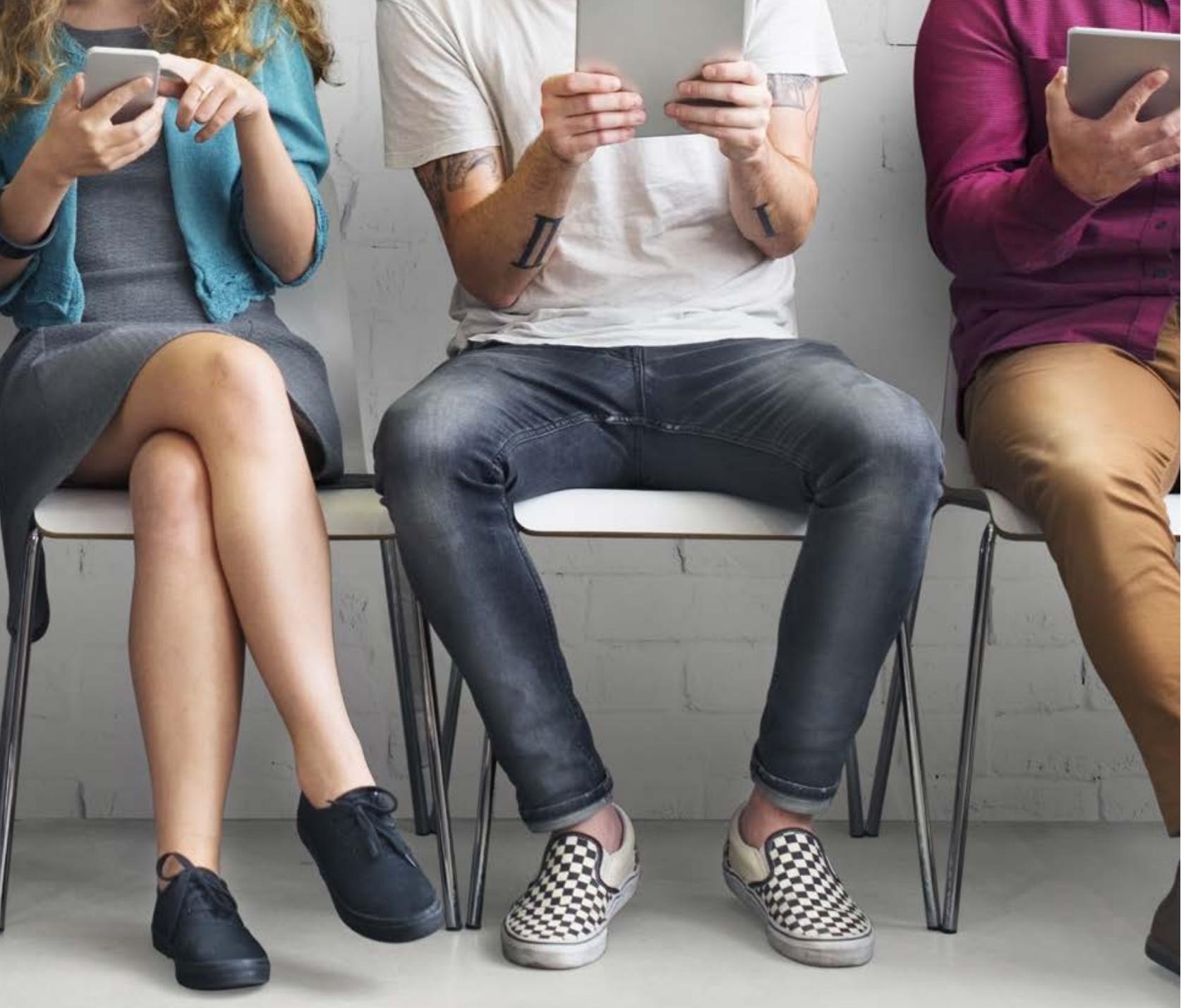

\section{Online usage inequalities}

Internet and Web-based health intervention use among people with different personal characteristics 
(C) Dominique Reinwand 2016

ISBN 9789461596352

Production Datawyse | Universitaire Pers Maastricht Cover picture (CRawpixcel.com by Fotolia

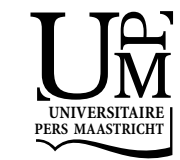




\title{
Online usage inequalities
}

\section{Internet and Web-based health intervention use among people with different personal characteristics}

\author{
Dissertation \\ to obtain the degree of Doctor at Maastricht University, \\ on the authority of the Rector Magnificus, Prof. dr. Rianne M. Letschert \\ in accordance with the decision of the Board of Deans, \\ to be defended in public \\ on Wednesday 21 December, at 12:00 hours
}

Dominique Alexandra Reinwand Born 5 February 1984 in Oberwesel (Germany) 


\section{Promotor}

Prof. dr. Hein de Vries

\section{Copromotor}

Dr. Rik Crutzen

\section{Assessment Committee}

Prof. dr. Nanne K de Vries (chair)

Prof. dr. Hans Bosma

Prof. dr. Lisette van Gemert-Pijnen, University of Twente

Prof. dr. Lilian Lechner, Open University

Dr. Anke Oenema

The research presented in this dissertation was conducted at the School for Public Health and Primary Care (CAPHRI), Department of Health Promotion, Maastricht University. CAPHRI participates in the Netherlands School of Primary Care Research (CaRe). Financial support for printing of this thesis was kindly provided by the University of Maastricht, the Netherlands. The PhD project was financially supported by a grant from the German foundation 'Wilhelm-Stiftung für Rehabilitationsforschung' provided by the Association for the Promotion of Science and Humanities in Germany.

All rights are reserved. No parts of this book may be reproduced or transmitted in any form or by any means, without the written permission from the author or, where appropriate, the publisher of the article 


\section{Contents}

Chapter 1 General Introduction

Chapter 2 Personal characteristics and trends across time of Internet use in the Netherlands: a multiwave panel study

Chapter 3 Website use and effects of online information about tobacco additives. A randomized controlled trial

Chapter 4 Who follows eHealth interventions as recommended? A study of participants' personal characteristics from the experimental arm of a randomized controlled trial

Chapter 5 Generating and predicting high quality action plans to facilitate physical activity and fruit and vegetable consumption: results from an experimental arm of a randomised controlled trial

Chapter 6 Impact of educational level on study attrition and evaluation of Webbased computer-tailored interventions: results from seven randomized controlled trials

Chapter 7 General discussion

References

Valorization Addendum

Summary

Samenvatting

Dankwoord

Curriculum Vitae

Publication List

Abbreviations 



\section{Chapter 1}

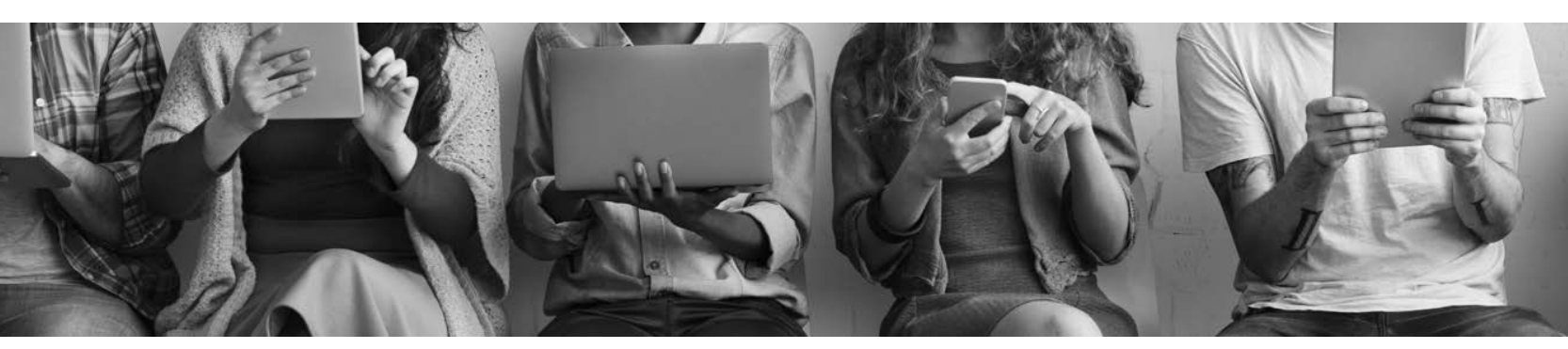

\section{General Introduction}




\section{GENERAL INTRODUCTION}

Socioeconomic differences are not only measurable as inequalities of education, income, and occupational status (American Psychological Association \& Task Force on Socioeconomic Status, 2007), they also affect health. Health inequalities among different socioeconomic groups are responsible for a large part of the total burden of diseases in Europe (Dahlgren \& Whitehead, 2007; Mackenbach et al., 2008). In the Netherlands, socioeconomic differences are also associated with variations in health and health behaviors. This results in an average of six years shorter life expectancy for people with a lower educational level compared to those with a higher educational level. Also, self-perceived good health is 19 years less for people with a lower educational level (Hoeymans et al., 2014).

Hence, there is a need to reduce health inequalities among people from different socioeconomic groups, for example by providing health information and health interventions. The Internet is a promising channel to deliver health information and health interventions to improve health behavior. In the past, several Web-based computer tailored interventions have shown very promising results regarding changing health behavior and improving health outcomes (e.g., Broekhuizen, Kroeze, van Poppel, Oenema, \& Brug, 2012; Kohl, Crutzen, \& de Vries, 2013). If Web-based information and interventions have an impact, these interventions should be used. Whether, the Internet and accordingly Web-based interventions are used differently by different subgroups is assessed within this dissertation.

Therefore, this chapter begins by providing an overview of health inequalities among people with different socioeconomic statuses (SES) and demographic characteristics. Subsequently, it outlines that the potential of the Internet to reach all subgroups and the benefits and potentials of Web-based computer tailored interventions for several health behaviors. This is followed by a description of the different Internet and Web-based intervention usage patterns for people with different sociodemographic characteristics. Afterwards, a main problem of these Web-based interventions, for people with different educational levels - dropout attrition - is explored and discussed. The chapter ends with a summary of the research questions and an outline of the dissertation.

\section{Socioeconomic variation and health inequalities}

A lower SES is associated with an unhealthy lifestyle and negative health outcomes (Adler \& Ostrove, 1999) such as lower physical activity (Gidlow, Johnston, Crone, Ellis, \& James, 2006), poor nutrition (Hanson \& Chen, 2007; Pampel, Krueger, \& Denney, 2010), higher smoking prevalence (Charafeddine, Demarest, Van der Heyden, Tafforeau, \& Van Oyen, 2013; Hiscock, Bauld, Amos, Fidler, \& Munafò, 2012), increased diabetes and obesity rate (Lampert, Kroll, von der Lippe, Müters, \& Stolzenberg, 2013; Monteiro, Moura, Conde, \& Popkin, 2004), higher likelihood of cancer (Merletti, Galassi, \& Spadea, 
2011), and a higher morbidity and mortality rate compared to people with a higher SES (Committee on Health and Behavior: Research Practice and Policy Board on Neuroscience and Behavioral Health, 2001; Mackenbach et al., 2008).

While differences in mortality rates between higher and lower socioeconomic groups have narrowed over the years, other health imbalances such as diabetes and obesity have become more prevalent among these groups (Bleich, Jarlenski, Bell, \& LaVeist, 2012; Mackenbach, 2006). The risk for some of the social health inequalities such as diabetes and obesity can be reduced, especially with a healthy lifestyle, but even in 2016, health inequalities are a problem in most European countries including the Netherlands (Poos, Burggink, \& van der Lucht, 2014).

Socioeconomic status is often assessed by measuring educational level, income, and occupational status (Lahelma, Laaksonen, Martinkainen, \& Rahkonen, 2008). Yet, other personal characteristics may play a role in the development of health inequalities, such as age, gender, and relationship status (Weyers, 2008). Taking these different personal characteristics into consideration may help to better understand health inequalities between different subgroups and adjust health information and health interventions accordingly to those specific groups. Therefore, these different sociodemographic characteristics and their relationships with online behavior are the focus of this dissertation.

\section{The Internet: access and possibilities}

At first glance the Internet is a promising tool for making health information and interventions accessible to a broad population from different social classes, since the majority of the inhabitants of the Netherlands have access (Miniwatts Marketing Group, 2015; Scoreboard, 2013). In particular, the "digital divide", which seperates people with higher and lower socioeconomic statuses in terms of access to the Internet, has been narrowed (CBS, 2014b; Koivusilta, Lintonen, \& Rimpela, 2007).

The Internet is an integral part of daily life and people use it for several reasons: communication, searching for information, entertainment purposes, banking, and shopping. But the Internet has also improved access to health related information, healthcare programs, and health-promoting interventions. In the Netherlands, $97 \%$ of the inhabitans have access to the Internet and $58 \%$ of those have used the Internet in the last three months to seek heath-related information (CBS, 2014b). The Internet provides health information for lay people, which is important because many people do not understand the medical terms and recommendations used by their physicans, but would like to gain more health-related knowledge (Kivits, 2006; McMullan, 2006). People who use the Internet to gain health-related information obtain a better understanding of their disease, are more confident in their patient-doctor relationship, are more compliant, and have better health outcomes (Hibbard \& Greene, 2013; Laugesen, Hassanein, \& Yuan, 2015). This shows the importance of the Internet to health promotion. 


\section{Web-based computer tailored health interventions}

Besides webpages providing health information, the Internet provides the opportunity to reach a wide audience with Web-based health interventions (Bennett \& Glasgow, 2009; Griffiths, Lindenmeyer, Powell, Lowe, \& Thorogood, 2006). These interventions are cognitive behavioral-based treatments, delivered via the Internet with the purpose of improving the health behaviors of target groups (L. Ritterband \& Thorndike, 2006; L. M. Ritterband et al., 2003). They are usually based on socio-cognitive theories, such as the Theory of Planned Behavior (Ajzen, 1991; Godin \& Kok, 1996), the Health Belief Model (Janz \& Becker, 1984), Self-Determination Theory (Deci \& Ryan, 2011; Ferguson, Gutberg, Schattke, Paulin, \& Jost, 2015), I-Change Model (de Vries, Mesters, van der Steeg, \& Honing, 2005; de Vries, van Osch, Eijmael, Smerecnik, \& Candel, 2012), and the Health Action Process Approach (Schwarzer, 1992; Schwarzer, Lippke, \& Luszczynska, 2011).

Behavior change strategies and advice are often tailored to participants' sociocognitive determinants and personal characteristics. Personalized advice and feedback created within Web-based computer tailored interventions has been shown to be evaluated as more personally relevant, and the information is seen as less redundant, and is more likely to be processed and remembered compared to generic information (Brug, Oenema, \& Campbell, 2003; Hawkins, Kreuter, Resnicow, Fishbein, \& Dijkstra, 2008; Lustria et al., 2013; S. Noar, Benac, \& Harris, 2007; te Poel, Bolman, Reubsaet, \& de Vries, 2009).

Web-based computer-tailored health interventions may have the potential to close the health inequality gap, because they are accessible online to a broad population. Numerous studies have demonstrated the effectiveness of such interventions in motivating individuals to adopt different health behaviors (Elbert et al., 2014; Kohl et al., 2013; Krebs, Prochaska, \& Rossi, 2010; M Neve, Morgan, Jones, \& Collins, 2010; S. Noar et al., 2007; S. Noar \& Harrington, 2012; S. M. Noar, Grant Harrington, van Stee, \& Shemanski Aldrich, 2011; G. J. Norman et al., 2007), including increasing physical activity (Ammann, Vandelanotte, de Vries, \& Mummery, 2013; Kroeze, Werkman, \& Brug, 2006; G. J. Norman et al., 2007; van 't Riet, Ruiter, Werrij, \& de Vries, 2010; van Stralen, de Vries, Mudde, Bolman, \& Lechner, 2011), healthy nutrition (Broekhuizen et al., 2012; Brug, Campbell, \& van Assema, 1999; Hamel \& Robbins, 2013; Krebs et al., 2010; Kroeze et al., 2006; Neville, O'Hara, \& Milat, 2009), smoking prevention and smoking cessation (de Jong de Josselin, Candel, Segaar, Cremers, \& de Vries, 2014; Elfeddali, Bolman, Candel, Wiers, \& de Vries, 2012; McClure et al., 2013; Smit, de Vries, \& Hoving, 2012), and reducing alcohol intake (Riper et al., 2008; Riper et al., 2011; Rooke, Thorsteinsson, Karpin, Copeland, \& Allsop, 2010). Moreover, interventions targeting multiple health behaviors have also been shown to be effective (Curioni \& Lourenco, 2005; Smeets, Kremers, De Vries, \& Brug, 2007; van Keulen et al., 2011; Vandelanotte, Reeves, Brug, \& De Bourdeaudhuij, 2008). Additionally, computer-tailored interventions are more cost- 
effective than typical health care measures (D. N. Schulz, Smit, et al., 2014; Smit, Evers, de Vries, \& Hoving, 2013; van Keulen et al., 2010).

\section{Second-level digital divide}

Web-based information and interventions are promising, and effective tools to improve health communication and education for a large and heterogenic population. However, the assumption that people with access to the Internet also obtain and use the information they need to make informed (health) decisions might be too optimistic. Having access to information and interventions does not automatically guarantee proper use. Nowadays, there is a second-level digital-divide. This refers to differences in Internet skills and usage (Hargittai, 2002). Differences in usage might be seen as a sort of new form of social inequality, which may result in differences in gained benefits from Internet usage, such as increased information (Zillien \& Hargittai, 2009). It is noticeable that people from different socioeconomic classes, with different personal characteristics use the Internet differently. This could be due to determinants such as different Internet skills patterns or different interests (van Deursen \& van Dijk, 2011, 2015).

Therefore, this dissertation starts by exploring whether people with different sociodemographic characteristics use the Internet differently in terms of searching for information, reading the news, watching movies, communicating, and playing games; and if there are detectible trends that could predict whether gaps these groups will widen or narrow in the future. Furthermore, it explores how much information individuals read on a website that provide health-related information. With this knowledge it will be possible to place health-related information tailored to online behavior patterns, to increase subgroup-specific information accessibility. Furthermore, this dissertation will explore the use of Web-based interventions by focusing on two different aspects: (1) the number of modules participants use within an intervention and, (2) the generation of action plans within such an intervention. Finally, dropout attrition, a major problem of Web-based interventions, will be evaluated. Understanding differences in usage patterns will allow researchers and intervention developers to target these interventions to potential subgroups.

\section{Differences in Internet usage}

Differences in Internet usage can be seen between individuals with different sociodemographic characteristics such as age, gender, educational level, income class, and occupational status.

Several studies have identified age as a significant factor. While the majority (80\%) of Dutch inhabitants aged 65 and older have access to the Internet (CBS, 2014c), they are less familiar with routine daily use (Hargittai, 2010; Olphert \& Damodaran, 2013; 
van Deursen \& Helsper, 2015). Elderly people are online less often and spend less time using the Internet (Dutton \& Blank, 2011). Older people who are online use the Internet more often for reading news and emailing (Howard, Rainie, \& Jones, 2001; R. B. Jones, Ashurst, Atkey, \& Duffy, 2015; S. Jones \& Fox, 2009), while younger people use it more often for entertainment purposes (e.g., playing games, watching movies) (Dutton \& Blank, 2011).

Gendered Internet use is also well reported. While older studies reported that females make less use of the Internet compared to males (Ching, Basham, \& Jang, 2005), recent statistics show that there is no difference in Internet access between men and women (CBS, 2014b). Nevertheless, it is known that males and females have different usage patterns. Females use the Internet more often than males to seek health-related information. On the other hand, males have been found to use the Internet to obtain information about product prices, sports news, and economic topics more often than woman (Debrand \& Johnson, 2008; Zillien \& Hargittai, 2009). Females spend more time online emailing and chatting for the purpose of long distance communication (Boneva, Kraut, \& Frohlich, 2001; Weiser, 2000) compared to males. Furthermore, young males tend to use anonymous chatrooms more often than females (Kalba, 2000; Wasserman \& Richmond-Abbott, 2005). Other studies, however, have not found gender differences with regards to online communication (Debrand \& Johnson, 2008).

People with a lower education level are online less frequently and spend less time online (Bonfadelli, 2002; CBS, 2014b), and vary in terms of online behavior in comparison with more educated individuals. People with a higher education make more use of emailing and chatting possibilities (Wasserman \& Richmond-Abbott, 2005). People with a lower education level have been found to use the Internet less often to search for information (S. Fox, 2011), but more frequently for entertainment purposes (i.e., gaming, watching movies) (Bonfadelli, 2002; CBS, 2014b; Hargittai, 2010; Livingstone \& Helsper, 2007).

Besides education level, income is also strongly associated with Internet use. People with a lower income are less likely to read online news (Madden \& Rainie, 2003) and other studies have found that people with a higher socioeconomic status (as indicated by income and occupation) have the highest pattern of information searching (Madden \& Rainie, 2003; Zillien \& Hargittai, 2009). People with lower incomes send emails less often (CBS, 2014b). In addition, people with a lower socioeconomic status use the Internet more superficially and less often to gain personal enhancement (i.e., educational purposes) (van Dijk, 2005). Furthermore, Dutch inhabitants with a lower incomes use the Internet to watch movies or play games less frequently, but make use of social networks like Facebook more often.

While several studies have examined personal characteristics and Internet usage, occupational status has received less attention. It is known that unemployed people spend more time online compared to employed people in their leisure time, playing 
more online games, and are more busy with communication (van Deursen \& van Dijk, 2014). On the other hand, employed people are more frequently online on a daily basis, often searching for information and news (CBS, 2014b).

With the exception of the finding that females spend more time online, similar Internet usage patterns among Dutch Internet users have been documented (Eurostat, 2015). These findings clearly demonstrate differences in Internet usage between individuals with different sociodemographic characteristics. Considering the narrowing digital divide, it seems likely that differences in Internet usage might also change over time. Knowing which subgroups are doing what activities online will help to present health-related online information to specific target groups. Examining this is part of the present dissertation.

\section{Differences in Web-based intervention usage}

Due to differences in online behavior, it can be assumed that there are also differences in the usage of Web-based health interventions among people with different personal characteristics. These differences such as the number of times a participant has access to an intervention, the time participants spend within an intervention, and the amount of completed modules of an intervention have been studied (Donkin et al., 2011). A review has found that people who started with a Web-based intervention module were younger, more likely to be female, and higher educated (E. Murray et al., 2013).

While some studies examine the effects of such interventions among subgroups like people with different educational levels, with indistinct results (e.g., Springvloet, Lechner, Vries, Candel, \& Oenema, 2015; Stanczyk, Bolman, van Adrichem, et al., 2014; van Genugten, van Empelen, \& Oenema, 2014; Walthouwer, Oenema, Lechner, \& de Vries, 2015), little attention has been paid to usage in terms of adherence to recommendations on how to use a Web-based intervention among subgroups so far. In order to achieve the desired behavior change, an intervention should be used as desired by the researcher (Donkin et al., 2013; Schweier et al., 2014). Studying revisits, logons, time spent using the intervention, and module completion might not be enough to understand who is doing what online and within those interventions. To be able to improve these interventions and target participants' characteristics, it is important to examine differences in intervention use. This might be helpful to understand which subgroups use the intervention as recommended and which do not, but this topic has received little attention in the literature so far and will be addressed in this dissertation.

\section{Dropout - a problem among Web-based health interventions}

The Internet and Web-based health interventions, also have their drawbacks. According to Eysenbach (Eysenbach, 2005), dropout, either not completing the study or missing follow-up measurements, is a "fundamental characteristic" of Internet interventions 
and a problematic issue. Dropout rates from Web-based health interventions can be over $80 \%$ in follow-up measurements (Melville, Casey, \& Kavanagh, 2010; Reinwand, Crutzen, et al., 2015). Eysenbach defined two kinds of dropout: non-completion or nonusage of the intervention and attrition from follow-up measurements. Both might have negative consequences for participants and researchers' non completion of the intervention might have consequences for the individual such as a less likely behavior change since studies have found a dose-response relationship between health behavior change and intervention participation (Donkin et al., 2013; Elfeddali, Bolman, Candel, et al., 2012; Vroege et al., 2014). The loss of participants to follow-up, dropout attrition makes analysis and statements of the effectiveness of these interventions more complicated and less valid when the outcome measured is missing at follow-up (Eysenbach, 2005). Christensen and Mackinnon (Christensen \& Mackinnon, 2006) raised the issue of insufficient research regarding dropout in 2006. Since then, findings about dropout among participants with different personal characteristics are still rarely reported and show ambiguity.

Findings from several reviews have found that younger participants drop out more frequently than older participants (Christensen, Griffiths, \& Farrer, 2009; Melville et al., 2010). With regards to gender, studies have revealed that males have a higher dropout rate compared to females (Melville et al., 2010; D. N. Schulz et al., 2012). Reviews have also demonstrated that participant's health status plays a role. Participants in Webbased mental health interventions with less severe symptoms have higher dropout rates (Melville et al., 2010). While some studies revealed that people with lower education levels have higher dropout rates from Web-based computer-tailored interventions than highly educated people (Geraghty, Torres, Leykin, Pérez-Stable, \& Muñoz, 2012; E. Murray et al., 2013; Price, Gros, McCauley, Gros, \& Ruggiero, 2012; Strecher et al., 2008), other studies have not found educational differences in terms of dropout (Christensen et al., 2009; Ezendam, Burg, Borsboom, van Empelen, \& Oenema, 2012; Habibović et al., 2014; Melville et al., 2010; E. Murray et al., 2013; Peel et al., 2012).

Possible reasons for dropout include intervention characteristics (e.g., workload, content), personal characteristics such as educational level, social support, or it can be related to participants' perceptions of the interventions, such as a lack of perceived benefits, which may result in dissatisfaction (Kelders, Kok, Ossebaard, \& Van GemertPijnen, 2012). Most of the mentioned studies found a relationship between socioeconomic status (often measured as educational status only) and attrition, either positive or negative. But these are only single studies and it would be beneficial to compare dropout rates within different Web-based computer-tailored interventions to be able to draw more valid conclusions about dropout and personal characteristics. If a similar pattern were to be proven for dropout in these interventions, it might be possible to find a common reason for this problem, which may narrow the problem in further Web-based interventions. 


\section{Aims and outline of this dissertation}

As the Internet is a medium with an important and irreplaceable role in health, it is important to understand who makes use of this resource and if there are less privileged groups that might be left behind.

Information simply about how much access people have to the Internet might be insufficient for public health researchers and Web-based intervention developers; it is important to know who does what online to be able to reach specific subgroups with Internet-delivered health information and interventions. Due to differences in online behavior, it can be assumed that there are also differences in the usage of Web-based health interventions among people with different personal characteristics.

Therefore, this dissertation assesses differences in Internet- and Web-based intervention usage, among people with different personal characteristics. It answers the following four research questions:

1. To what extent does Internet usage differ among people with different personal characteristics, and if so, and what are the trends over time for different Internet activities?

In order to answer these research questions, Chapter 2 of this dissertation provides an overview of different Internet activities (searching information, reading news, visiting forums, emailing, being active in social medias, watching movies, gaming, and how many hours are spent on each activity) from 2009 until 2014 and discusses the trends of Internet usage for different subgroups with regards to age, gender, educational level, income and working situation (Reinwand, Candel, Crutzen \& de Vries, 2016 submitted).

2. To what extent are there differences in health-related website usage among people with different personal characteristics?

The second research question is discussed in Chapter 3. It focuses on online behavior when individuals are asked to visit a website that provides information about tobacco additives, and elaborates on the differences in website use and information seeking among the mentioned subgroups (Reinwand, Crutzen, Talhout, Kienhuis, \& de Vries, 2016 submitted).

3. To what extent are personal characteristics associated with Web-based computertailored health intervention usage as recommended by intervention developers?

This research question is answered in Chapter 4 and 5 . Whether different subgroups adhere to recommendations and participate in the advised number of online modules within a Web-based computer-tailored intervention is discussed in Chapter 4 (Reinwand, Schulz, Crutzen, Kremers, \& de Vries, 2015). Chapter 5 describes the usage of a Web-based application for the generation of action plans within a computertailored intervention study (Reinwand et al., 2016). 
4. What role does education level play in dropouts from Web-based computertailored health interventions?

Chapter 6 discusses the last research question and focuses on dropout attrition from seven different computer tailored Web-based interventions, the role of education level, and appreciation of the interventions (Reinwand, Crutzen, et al., 2015).

Finally, in Chapter 7, a general discussion about these findings is summarized and discussed, and the theoretical and practical implications of the current dissertation are outlined.

Table 1.1: Overview of studies presented in this dissertation

\begin{tabular}{|c|c|c|c|c|}
\hline Chapter & Focus dissertation & Study subject & Participants & Sample Size \\
\hline Two & Internet usage & $\begin{array}{l}\text { Trend analysis of Internet usage } \\
\text { between 2009-2014 among } \\
\text { people with different } \\
\text { sociodemographic } \\
\text { characteristics }\end{array}$ & $\begin{array}{l}\text { Representative sample of } \\
\text { the general Dutch } \\
\text { population }\end{array}$ & $\begin{array}{l}N \text { ranges in the } \\
\text { six waves from } \\
5896-6598\end{array}$ \\
\hline Three & Website usage & $\begin{array}{l}\text { Effects of visiting an educational } \\
\text { website about tobacco additives }\end{array}$ & $\begin{array}{l}\text { Stratified sample of } \\
\text { smokers and non-smokers } \\
\text { from the general Dutch } \\
\text { population }\end{array}$ & $N=672$ \\
\hline Four & $\begin{array}{l}\text { Web-based } \\
\text { computer-tailored } \\
\text { intervention usage }\end{array}$ & $\begin{array}{l}\text { Web-based computer-tailored } \\
\text { intervention aiming to improve } \\
\text { five different health lifestyle } \\
\text { behaviors }\end{array}$ & General Dutch population & $\begin{array}{l}\text { Intervention arm: } \\
\mathrm{n}=1638\end{array}$ \\
\hline Five & $\begin{array}{l}\text { Web-based } \\
\text { computer tailored } \\
\text { intervention usage }\end{array}$ & $\begin{array}{l}\text { Web-based computer-tailored } \\
\text { planning intervention aimed at } \\
\text { generating action and coping } \\
\text { plans to improve fruit and } \\
\text { vegetable consumption and } \\
\text { physical activity. }\end{array}$ & $\begin{array}{l}\text { General Dutch and German } \\
\text { populations }\end{array}$ & $\begin{array}{l}\text { Intervention arm: } \\
\mathrm{n}=346\end{array}$ \\
\hline Six & $\begin{array}{l}\text { Study dropout from } \\
\text { Web-based } \\
\text { computer-tailored } \\
\text { interventions }\end{array}$ & $\begin{array}{l}\text { Comparing dropout attrition for } \\
\text { people with different } \\
\text { educational level from seven } \\
\text { different Web-based computer- } \\
\text { tailored interventions }\end{array}$ & $\begin{array}{l}\text { Differs per study: general } \\
\text { Dutch or German } \\
\text { population, smokers and } \\
\text { non-smokers, people with } \\
\text { COPD, and normal and } \\
\text { overweight individuals }\end{array}$ & $\begin{array}{l}\mathrm{N} \text { ranges in the } \\
\text { seven studies } \\
\text { from } 1149-5055\end{array}$ \\
\hline
\end{tabular}




\section{ABSTRACT}

Background: Unequal internet use bears the risk that some subgroups might be deprived from important information and possibilities offered by the Internet. This paper describes the trends across time in seven different online activities from 2009 2014 and differences in trends according the person characteristics age, gender, education, income, and employment status.

Methods: We used longitudinal data from six waves (2009-2014 ( $N=20.763))$ from a panel study. Trend analyses were done employing linear and logistic mixed regression models.

Results: All Internet activities increased in use except for being active in forums/blogs which decreased. Information seeking and emailing are the most popular online activity. Activities regarding social interaction (mailing, social network websites), entertainment (gaming, movies), and activities for personal development (reading news) increased but differences between subgroups exist.

Discussion: Differences in Internet activities between subgroups exist. Further research is necessary to find reasons for these gaps in online behavior.

Keywords: Internet use, panel study, general population, sociodemographic characteristics, trend analysis, digital divide, 


\section{INTRODUCTION}

We can no longer imagine everyday life without the Internet. It is an integral part of our daily lives and the way in which we communicate and search for information. We use it for entertainment purposes, banking and shopping, as well as for health purposes. In the early days of the Internet, the inequality between different socio-economic groups in terms of access to the Internet was called the "digital divide" (Compaine, 2001). In particular people from lower socio-economic backgrounds were deprived of the important information and possibilities offered by the Internet (Koivusilta et al., 2007).

Today, the gap between connected and disconnected people is being closed in terms of Internet access. The majority of people have Internet access in Western countries, especially in the Netherlands, where the current study takes place (Scoreboard, 2013; Statistics Netherlands, 2012). However, there is still inequality with regards to Internet usage behaviors that are observable for people who differ in personal characteristics such as age, gender, educational level, income, and employment status. This inequality does not necessarily concern access to the Internet, but rather the way in which the Internet is used (van Deursen \& van Dijk, 2011, 2015).

Several studies have identified age differences with regards to Internet use. In general, older people are less often online and spend less time using the Internet (Dutton \& Blank, 2011). Older people who are online use the Internet more often for reading news and emailing (Howard et al., 2001; R. B. Jones et al., 2015; S. Jones \& Fox, 2009), while younger people use the Internet more often for entertainment purposes (e.g., playing games and watching movies) (Dutton \& Blank, 2011).

Gendered Internet use is also commonly reported. While older studies have reported that females make less frequent use of the Internet compared to males (Ching et al., 2005), recent statistics demonstrate that there is no difference between the genders in terms of Internet access (CBS, 2014b). Nevertheless, it is known that males and females have different Internet usage patterns. Females use the Internet more often to seek health related information compared to males. On the other hand, males have been found to use the Internet more often than females in order to acquire information regarding product prices, sports news, and economic (Debrand \& Johnson, 2008; Zillien \& Hargittai, 2009). Females spend more time online emailing (Boneva et al., 2001; Weiser, 2000) compared to males. Other studies, however, did not find gender differences with regard to online communication (i.e., emailing) (Debrand \& Johnson, 2008).

Several studies have indicated that people with a lower educational level are less frequently online and spend less time online (Bonfadelli, 2002; CBS, 2014b) and are different in terms of online behavior. Individuals with higher educational levels more often make use of emailing possibilities (Wasserman \& Richmond-Abbott, 2005). People with lower educational levels have been found to use the Internet less often to search for information compared to higher educated people (S. Fox, 2011), but more 
frequently use it for entertainment purposes (i.e., games) (Bonfadelli, 2002; CBS, 2014b; Hargittai, 2010; Livingstone \& Helsper, 2007).

Besides educational level, income is also strongly associated with Internet use. Traditionally, people with a higher income more often had access to the Internet, but this gap has nearly been closed now: $93 \%$ of the lowest income groups had Internet access in the Netherlands in 2013 compared to 99\% of Dutch inhabitants from the highest income group (CBS, 2014b). People with a lower income are less likely to read news online (Madden \& Rainie, 2003). Other studies have investigated that people with a higher socioeconomic-status (SES) (as indicated by income and occupation) more often search online information compared to people with a lower SES (Madden \& Rainie, 2003; Zillien \& Hargittai, 2009). People with a lower income also less often send emails (CBS, 2014b). In addition, people with a lower socio-economic status use the Internet more superficially and less often for personal enhancement (i.e., educational purposes) (van Dijk, 2005).

While several of the studies cited above have investigated the relationship between personal characteristics and Internet use, occupational status has received less attention in research to date. It is known that unemployed people spend more time online compared to employed people in their leisure time, playing more online games and using it for communication purposes (van Deursen \& van Dijk, 2014). On the other hand, it is known that employed people are more frequently online on a daily basis, more often searching for information and news (CBS, 2014b).

These differences in Internet use can lead to inequalities. People who do not use the Internet in order to develop personal skills for certain purposes, such as seeking (health) information, reading news, looking for job vacancies, comparing prices, or e-learning, might be disadvantaged and unable to make use of the benefits that the Internet provides (DiMaggio \& Hargittai, 2001). Furthermore, this kind of digital exclusion might even widen the gap between people with differing SES (Ewa, 2010; Livingstone \& Helsper, 2007).

Although the importance of inequality has been acknowledged, most studies cited here have a cross-sectional design, which only represents a snapshot of Internet use. Longitudinal trend analyses are scarce in the literature. To our knowledge, only one study has assessed Internet use across four years in the Netherlands among four different subgroups, and this study confirmed that people with a lower educational level used the Internet less often for personal development and that the gaps between people with different socioeconomic-characteristics are not decreasing (van Deursen, van Dijk, \& ten Klooster, 2015). Longitudinal studies considering different online activities, different personal characteristics, and the time people spend online engaging in several activities could demonstrate developments over time and whether usage gaps are changing between subgroups. This information could be used to take action in order to support digital inclusion for identified target groups (e.g., including Internet skills in the school curriculum). 
Therefore, this paper aims firstly, to describe the trends of seven different online activities (i.e., searching for information, reading news, visiting and writing on forums/blogs, using email, visiting social media websites, watching movies, and playing games) over six years (2009-2014) and, secondly, to describe differences in these online activities among subgroups based on the variables of age, gender, education, income and employment over time.

\section{METHODS}

In order to investigate Internet use from 2009 until 2014, we made use of data in six waves from the Dutch Longitudinal Internet Studies for the Social Sciences (LISS) household panel. The LISS panel is a representative sample of Dutch individuals who participate in monthly Internet surveys. The panel is based on a true probability sample of households drawn from the population register. Households that could not otherwise participate are provided with a computer and/or Internet connection. A longitudinal survey takes place annually in the panel, including a variety of domains such as work, education, income, housing, and leisure time (Schwerpenzeel \& Das, 2010). For more information, visit: www.lissdata.nl.

The panel consists of approximately 7000 individuals aged 16 and older who complete monthly online questionnaires on different topics. Each questionnaire takes approximately 15 minutes and participants received rewards for filling in each questionnaire. For this study, the relevant information is taken from the "Social Integration and Leisure" questionnaire combined with the "Background Variables", which are part of the LISS core study. Since 2008, annual waves focusing on these topics have taken place. The "Social Integration and Leisure" questionnaire is annually assessed in February and March. Background variables, which include all of the demographic characteristics of the participants, are assessed monthly. Data were collected and administered by CentERdata (Tilburg University, The Netherlands) and have also obtained ethical approval (www.centerdata.nl/en).

\section{Measurements}

From the "Background Variables" questionnaire, we used gender, age, education, income, and employment status for this study. Gender is measured dichotomously $(0=$ male, $1=$ female). Age is categorized into four groups $(1=<29,2=30-44,3=45-64$, $4=>65$ years). Education was assessed by asking for the highest obtained educational level and was categorized into three groups: lower ( $1=$ no education, primary or lower vocational school), middle (2=secondary vocational school or high school), and higher (3=higher professional education or university) educational level (Luijkx \& de Heus, 2008). On the basis of the personal net monthly income in Euros, income was 
categorized into three groups: low $(1=<€ 1532)$, middle $(2=€ 1533-€ 2481)$, and high (3=>€2482) (van den Brakel \& Ament, 2010). If personal net monthly income has missing values, it has been imputed on the basis information regarding gross income, if available; the equation for this is described elsewhere (de Voss, 2011). Employment status was divided into employees $(=2)$, which are individuals with any type of paid employment, and unemployed respondents $(=1)$. The latter group consists of students, pensioners, people responsible for doing the housekeeping, people seeking a job or being work disabled, and people who reported doing something else.

As indicators for Internet use behavior from 2009 until 2014, we used the following seven variables from the annual "Social Integration and Leisure" questionnaire: "Can you indicate whether you ever spend time on the following online activities?" (1) "Searching information on the Internet", (2) "Reading news online", (3) "Visiting and or writing on forums or blogs", (4) "Emailing", (5) "Visiting social network sites like Facebook or others" (included as of 2012), (6) "Watching movies", and (7) "Playing online games". From 2008 to 2011, watching movies was assessed with two items "Watching short films" and "Watching online films" -but since 2012, one item was used: "Watching online films". For our analysis, we combined the two items as measured up to 2011 into one item. All questions could be answered with yes $(=1)$ or no $(=2)$. In addition, those participants that performed an online activity were asked to estimate how many hours per week, on average, they spent on this activity. Trends in the number of persons spending time on a particular online activity as well as the amount of time spent weekly by these persons on this activity were analyzed.

\section{Statistical analysis}

Data analysis was undertaken with SPSS 22 (IBM Corp, NY, USA). Descriptive statistics were calculated in order to describe the study sample for the waves from 2009 to 2014. To answer the research questions, we made use of the general linear mixed model. This analysis technique has the advantage that data from individuals who skipped one or more waves remain in the analyses with regards to the other waves (Heck, Thomas, \& Tabata, 2012).

At first, we investigated change over time for the seven different online behaviors and the associated time spent performing these behaviors online respectively as dependent variables. This involved two-level models with repeated measurements (level 1) nested within participants (level 2). In all cases we tested for linear, quadratic $\left(\right.$ time $^{2}$ ), and cubic (time ${ }^{3}$ ) time trends, including age, gender, level of education, income, and employment status as covariates. Using a top-down procedure, we compared the values of -2 log likelihood from the model without any specific trend with the model with the highest polynomial trend (cubic) and tested for significance by employing the $\chi^{2}$-distribution, as a reference distribution with degrees of freedom equal to the difference in the numbers of parameters of both models. If this comparison was 
significant, we used a model without any specific trend for the following analyses. If the comparison was not significant, we checked if the cubic component of the polynomial trend was significant in the model, and if so, we used the cubic time trend model. If the cubic component was not significant in the model, we deleted the term and checked the significance of the quadratic term. Again, if this term was significant, we used the quadratic term in the following analysis, if this term was also not significant, we used the linear time trend model. All subsequent analyses were conducted with the time trend as determined in the above way.

For the primary analysis, two-level models with repeated measurements nested within participants were also analyzed in order to investigate differences in online behavior over time between the subgroups as based on the variables age, gender, education, income, and employment status. First, a model was fitted in which, next to the trend model as determined previously, the background variables (age, gender, income, education, and employment) were added, as well as interaction terms between wave and these background variables. Again, a top-down testing procedure was followed, in which the first potential moderation effects that is, interaction terms of the trend for wave with each of the background variables were tested for significance. We excluded one by one the interaction term with the highest $p$-value from the model until only significant interaction terms remained. If a significant interaction term was present in the final model, separate analyses were performed for the subgroups as defined by the corresponding background variable involved in the interaction. In these cases, dummy variables for age, education, and income were used in order to obtain results for each of the separate groups, where the lowest category $(2009$, age $<29$, male, lower level of education, lower level of income, and unemployed) was defined as being the reference group. P-values $\leq .05$ were considered to be statistically significant. These analyses were conducted for all seven online behaviors (logistic mixed regression) and for all seven corresponding times (hours per week) spent performing these activities (linear mixed regression). In order to accommodate dependencies across time in respondents' behavior, the covariance matrix of the random time effects was assumed to satisfy a Toeplitz structure in case of the logistic model, and a random intercept model for the linear model.

\section{RESULTS}

Table 2.1 presents the sample characteristics of the panel members who participated from 2009 until 2014. 
Table 2.1: Study sample characteristics, $2009-2014$

\begin{tabular}{|c|c|c|c|c|c|c|}
\hline & 2009 & 2010 & 2011 & 2012 & 2013 & 2014 \\
\hline & $N=5896$ & $N=6377$ & $N=5625$ & $N=5971$ & $N=5721$ & $N=6598$ \\
\hline & n (\%) & $\mathrm{n}(\%)$ & $\mathrm{n}(\%)$ & $\mathrm{n}(\%)$ & n (\%) & $\mathrm{n}(\%)$ \\
\hline \multicolumn{7}{|l|}{ Age } \\
\hline$<29$ & $1068(18.1)$ & 1122 (17.6) & $927(16.5)$ & $938(15.7)$ & $894(15.6)$ & $1176(17.8)$ \\
\hline $30-44$ & $1584(26.9)$ & $1566(24.6)$ & $1299(23.1)$ & $1340(22.4)$ & $1228(21.5)$ & $1439(21.8)$ \\
\hline $45-64$ & $2397(40.7)$ & 2540 (39.8) & $2280(40.5)$ & $2386(40.0)$ & 2255 (39.4) & $2442(37.0)$ \\
\hline$>65$ & $847(14.4)$ & 1149 (18.0) & 1119 (19.9) & 1307 (21.9) & $1344(23.5)$ & $1541(23.4)$ \\
\hline \multicolumn{7}{|l|}{ Gender } \\
\hline Male & 2708 (45.9) & $2964(46.5)$ & $2589(46.0)$ & $2784(46.6)$ & $2645(46.2)$ & $3042(46.1)$ \\
\hline Female & $3188(54.1)$ & $3413(53.5)$ & $3036(54.0)$ & $3187(53.4)$ & 3076 (53.8) & $3556(53.9)$ \\
\hline \multicolumn{7}{|l|}{ Education* } \\
\hline High & $1731(29.4)$ & 1893 (37.1) & 1715 (30.0) & $1840(35.4)$ & 1766 (31.0) & $2130(32.4)$ \\
\hline Middle & $1980(33.6)$ & 2104 (33.1) & $1867(33.3)$ & 2007 (33.7) & 1960 (34.4) & $2329(35.4)$ \\
\hline Low & $2183(37.0)$ & 2359 (29.8) & 2029 (36 2) & 2109 (30.9) & 1978 (34.7) & $2117(32.2)$ \\
\hline \multicolumn{7}{|l|}{ Income* } \\
\hline High & $628(12.7)$ & $697(13.0)$ & 611 (12.9) & $685(13.6)$ & $647(13.5)$ & 765 (13.9) \\
\hline Middle & 1649 (33.3) & $1885(35.1)$ & $1690(35.6)$ & $1814(36.0)$ & $1762(36.8)$ & $1985(36.0)$ \\
\hline Low & $2677(54.0)$ & 2795 (52.0) & 2442 (51 5) & $2538(50.4)$ & 2378 (49.7) & $2759(50.1)$ \\
\hline \multicolumn{7}{|c|}{ Working situation } \\
\hline Unemployed & 2407 (40.8) & $2856(44.8)$ & 2527 (44.9) & 2721 (45 6) & 2681 (46.9) & $3133(47.5)$ \\
\hline Employment & 3489 (59.2) & $3521(55.2)$ & 3098 (55 1) & $3250(54.4)$ & $3040(53.1)$ & $3465(52.5)$ \\
\hline
\end{tabular}

*Missing values for income and educational level are possible since these are based on voluntary information.

Table 2.2 summarizes the results regarding whether individuals use the Internet for the specific behaviors being assessed, and Table 2.3 presents the results regarding the times individuals spend online performing each of these behaviors. Figures $2.1-2.7$ show, per online activity, the trends over time regarding usage and the average time spent performing the behaviors beings assessed.

The trend analysis revealed that we did not have to use a specific time trend, except for the analysis on the average time spent being active on social media websites, where we used a linear trend model. The following description of the findings is limited to significant results. If present, we will describe the interaction effects otherwise, main effects are described.

\section{Age}

The trend in the percentage of people who search online for information does not differ significantly between age groups. The trend seems to be rather stable on average (see 
also Figure 2.1), so the gap between younger and older people persists. Furthermore, the higher the age, the smaller the percentage of persons that search for information on the Internet. In addition, the trend in time spent on the Internet on searching for information does not differ between age groups. Compared to 2009, there seems to be a slight increase over time, but only significantly so in 2011. Furthermore, there is a negative relationship between age and time spent searching for information; the oldest age group compared to the age group of less than 29 years spent on average, one hour less per week on this particular activity.

Regarding reading online news, the trend across time differs between age groups. Compared to 2009, reading online news increased significantly in all age groups, except for the group with people aged older than 65 years, where the trend is approximately stable (Figure 2.2), and the gap between this age group compared to other age groups has become larger. The time spent reading online news did not differ between age groups, and since 2009, there has been a slight increase in time spent on this activity, with significant increases in 2011, 2013, and 2014.

Over time, in all age groups, after an increase in the percentage of people being active on online forums/blogs, there has been a decrease in recent wave(s) compared to 2009. Furthermore, the time spent online on this activity consistently decreases with significant changes in 2013 (on average 0.8 hours less per week) and 2014 (on average 1.3 hours less per week). This trend is similar for all age groups.

The trend across time for the percentage of people using the Internet for emailing does not differ significantly between age groups, and this seems to be a stable trend (Figure 2.4). However, with increasing age, the percentage of people using email decreases significantly. In terms of the time spent online using emails, it can be seen that the older the people, the less time they spend on this activity. For example, people older than 65 years spend, on average, 1.3 hours less per week emailing compared to people younger than 29 years.

The trend in the percentage of people being active on social networking websites does not differ significantly between age groups, and this seems to be a stable trend. It can be seen that the higher the age, the less likely it is that people are active on social networking websites. The linear trend in the time spent on this online activity also does not differ between the age groups. Since 2012, the time per week spent on social websites has increased, and there is a significant negative relationship between age and weekly time spent online on social networking websites. For example, people aged 3044 years and older spend 1.6 hours per week less on this activity compared to people younger than 29 years.

Similar to for reading online news, there is a different time trend in terms of the percentage of people watching online movies between age groups. However, within all age groups, there has been an increase in this activity, with significant peaks in 2012 and 2013 and a decrease in 2014. Figure 2.6 shows that the younger the age group, the higher the percentage of people watching movies online. In terms of time spent online 
watching movies, the trend over time is similar for all age groups. Compared to 2009, there has been a constant increase in hours per week spent watching online movies. Again, the older the age group, the less time they spend on this activity.

The trend across time in terms of the percentage of persons playing online games is different for different age groups. Over time, there has clearly been an increase in the number of persons aged 30-44 and 45-64 years who play online games. For the youngest and oldest age groups, this trend is rather stable, and it seems that the gap between the oldest age group and people aged 30-44 and 45-64 years has become larger. In terms of the time spent online performing this activity, the trend is approximately the same for all age groups. The average time seems to increase gradually from 2009 onwards, becoming significantly greater in 2014 compared to 2009.

\section{Gender}

Neither the trend over time in the percentage of people searching online for information nor the trend over time for the amount of time spent on this activity differ significantly between genders. Compared to males, females spend on average 0.3 hours per week less online searching for information.

The same pattern holds for the activity of reading online news and being active on forums/blogs. There is no difference over time between males and females. The percentages of females reading news online and being active on forums/blogs are significantly lower, and these trends seem to be stable. Females also spend significantly less time online reading news compared to males.

The trend across time in terms of writing and receiving emails differs between males and females. Over time, there has been an increase for both genders, with significant peaks in 2012 for males and in 2014 for males and females compared to 2009.

The trend in the percentage of people being active on social networking websites and the trend across time regarding the amount of time spent online on this activity do not significantly differ between males and females. Compared to males, more females state that they use these websites, and this seems to be stable. Females also spend significantly more time per week (0.5 hours) online on this activity (Figure 2.5 ).

Over time, there is a clear difference between the percentages of males and females watching online movies. For both groups, there has been constant growth since 2009 up to 2013, with peaks in 2012 and 2013, especially for males. In 2014, there was a decrease for both males and females. Regarding the time spent online watching movies, the trend over time is also different for males and females. Compared to 2009, males have significantly increased the hours spent watching online movies since 2012. For females, a significant increase in time spent watching online movies has been found only in 2014 compared to 2009.

The trend across time in terms of the percentage of people playing games online differs between genders. Over time, there has been a significant increase since 2012 for 
both genders, with the trend being somewhat stronger for females. There has been a slight increase over the recent years regarding the amount of time that males and females spend online playing games. On average, females spend less time (0.6 hours per week) online performing this activity.

\section{Educational levels}

The trend over time for the percentage of people as well as the trend over time regarding the amount of time spent weekly online searching for information are the same for persons with different levels of education. There is a significant and stable positive relationship between the level of education and searching for information online in terms of both the percentage of persons and the average amount of time spent performing this activity.

The trend across time in terms of the percentage of persons reading online news is different for different educational levels. Over time, there is a significant increase for all three educational groups, but it can be seen that people with a lower educational level only show increases in 2013 and 2014, whereas other educational level groups show increased percentages from 2010 and 2011 onwards. In terms of the time spent online reading news, the trend is similar for all educational level groups.

The trend over time in terms of people being active on forums/blogs did not significantly differ between educational groups, but for all waves people with higher and middle educational levels were more likely to be active on forums/blogs than people with a lower educational level. Regarding the trend over time in terms of the amount of time people spent weekly on forums/blogs, we have found differences between the educational level groups. For the lower and middle educated people, the average time per week people spent on this activity decreased over time. People with a lower level of education spent the most time online on forums/blogs, with a significant decrease in time found in 2013 and 2014 compared to 2009. For middle educated people, the weekly time spent reduced significantly, with 0.7 hours per week in 2014, whereas for the highly educated group, there seems to have been a slight increase since 2009.

The time trend concerning the percentage of people using the Internet for emails is different between the educational level groups. For all groups, an increase can be seen, and the largest increase was for people with a higher educational level, while the smallest increase was for people with a lower level of education. Compared to 2009, significant differences can be seen for higher educated people in 2012 and 2014; for people with middle education the increase in percentage is significant in all years except for in 2013. The slight increase for people with lower education does not reach significance. Compared to people with a lower educational level, middle and highly educated people spent significantly more time online using email. The higher the education, the more time they spent on this activity (Figure 2.4). 
The trend in the percentage of persons that use social networking websites differs between educational level groups. Compared to 2012 significantly more people used these websites in 2013 and 2014 in all educational level groups. The highest increase in 2014 was found among people with a higher educational level. There were no differences between educational level groups in terms of trends across time concerning the amount of time spent on social networking websites. The higher the educational level, the less time people spent online on this activity. Highly educated people spent on average, 0.85 hours per week less online compared to lower educated people.

Concerning watching online movies, the trend over time in terms of the percentage of people using the Internet for this activity differs between the educational level groups. Over time, there first is an increasing trend for all educational groups and then, in 2014 a small decrease. Regarding the trend across time in terms of the amount of time spent watching movies, no differences between the three educational level groups can be seen. There is a negative relationship between educational level and the amount of time people spent watching online movies. Highly educated people spent significantly less time watching movies, spending on average 0.40 hours per week less on this activity than people with a lower educational level.

Neither the trend over time in terms of in the percentage of people playing online games nor the trend over time regarding the amount of time spent on this activity differ significantly between educational level groups. The higher the educational level, the less people play online games and the less time per week people spend on this activity.

\section{Income levels}

The trend over time regarding the percentage of people searching for information online as well as the trend over time regarding the amount of time spent per week on this activity are the same for different income levels. There is a significant positive relationship between income and the percentage of people searching for information online. The same is true of the time people spend on this activity. People with a high income and middle income spend, respectively, on average 0.3 hours and 0.2 hours per week more for this activity compared to people with a lower income.

Similar findings can be reported concerning the activity of reading online news. The trend across time is similar for the three income level groups in terms of the percentage as well as the amount of time spent reading online news per week. Significantly more people with a high and middle income level read news online compared to people with a lower income level.

Regarding the activity of being active on forums/blogs, no significant differences could be found with regards to the subgroups of income, nor for the trend over time regarding the percentage of people performing this online behaviour nor for the amount of time spent on this activity. 
The trend across time in terms of the percentage of writing emails is different for different income level groups. Only within the middle income group could a significant increase be seen from 2011 to 2014, when compared to 2009. The trend seems to be relatively stable within low and high income groups, while the gap between low and middle levels of income widened in 2014. In terms of time spent online, the trend over time is the same for all income level groups. Compared to people with a low income, people from middle (1.2 hours) and high ( 2.4 hours) income groups spent significantly more time per week online writing emails.

The trend across time in terms of the percentage of people being active on social networking websites is similar for different income level groups, and there is also no difference over time regarding the amount of time spent on this activity. Significantly more people with a higher income use social networking sites compared to people with a lower income, and this gap persists over time. Furthermore, people with a middle income spend significantly less time on these websites compared to people with a lower income.

Similar usage patterns can be described concerning watching movies. Here, the trend over time is also similar for different income groups in terms of the percentage and time spent on watching movies. The higher the level of income, the lower the percentage of people watching movies online. Moreover, people with a higher level of income spend significantly less time online per week engaging in this activity compared to people with a lower level of income (Figure 2.6).

The trend across waves in terms of the percentage of persons playing online games is different for different income level groups. Over time, there has been an increase in people playing games within all three groups from 2012 to 2014 when compared to 2009. The increase is comparable and steady for both the lower and middle income groups until 2014. Since there was no significant difference in 2009 between the lower and middle income groups, this suggests that there is hardly a gap between these groups. In terms of the time spent online, the trend over time is not significantly different for the different income level groups. People with a higher income spend significantly less time online playing games compared to people with a lower income.

\section{Employment}

Neither the trend over time in the percentage of people searching online for information nor the trend over time for the amount of time spent on this activity differ significantly between unemployed and employed people. Significantly more employed people search for information online compared to unemployed people.

Similarly to searching for information, no significant differences in the trends over time in the percentage of people reading online news and being active on social networking websites could be found between the two employment groups, and this was also the case for the trends over time for the amount of time spent online 
regarding these two activities. Significantly more employed people are active on social networking websites, but on average they less time per week online compared to unemployed people.

Regarding being active on forums/blogs, there was also no difference in trend over time for the percentage of employed and unemployed people engaging in this activity. However, there is a difference across time concerning the weekly time spent on this activity. For both groups, the trend was decreasing. When compared to 2009, employed people spent in 0.4 hours less per week online in 2013, and unemployed people spent 0.8 hours less per week online in 2014.

The trend over time for the percentage of people writing emails was similar for employed and unemployed people, but the trend over time regarding the amount of time regarding the amount of time spent writing emailing differs between these groups. For unemployed people, there is stable trend across time regarding the amount of time spent writing emails. This contrasts with employed people, who, since 2012 onwards spent significantly more time writing emails.

The trend over time regarding the percentage of people watching online movies differs between unemployed and employed people. In both groups the percentage increased significantly compared to 2009, for employed people, this trend has been observed since 2010, and for unemployed people, this trend has been observed since 2012. The trend in time spent watching online movies is the same for both groups. Employed people spent significantly less time watching online movies ( 0.40 hours per week) compared to unemployed people.

The trend regarding the percentage of persons who play online games does not differ significantly between unemployed and employed people. Compared to unemployed people, a smaller percentage of employed people play games online (Figure 2.7). In addition, the trend over time concerning the time spent per week playing games did not differ between these two groups. 


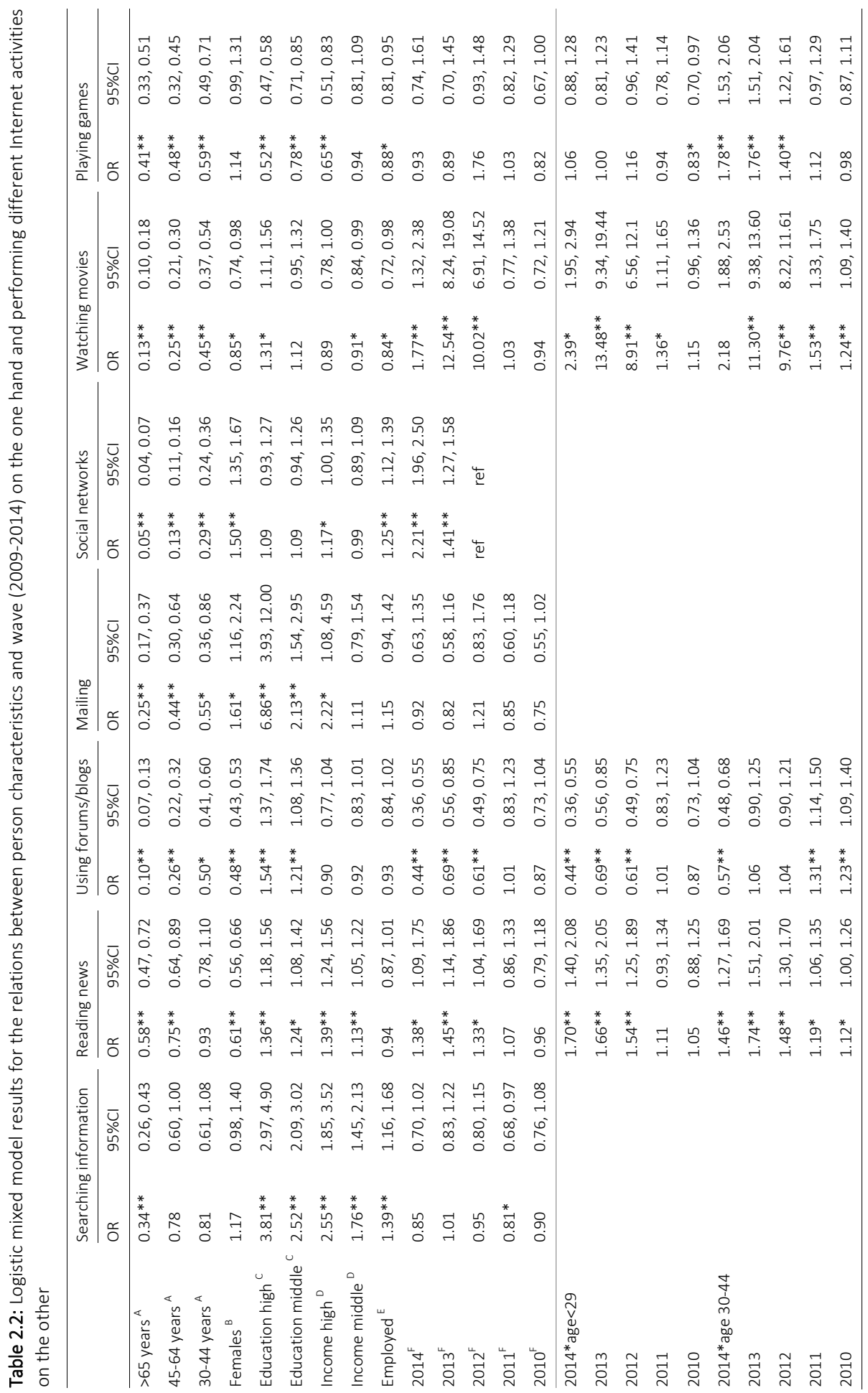




\section{Chapter 2}

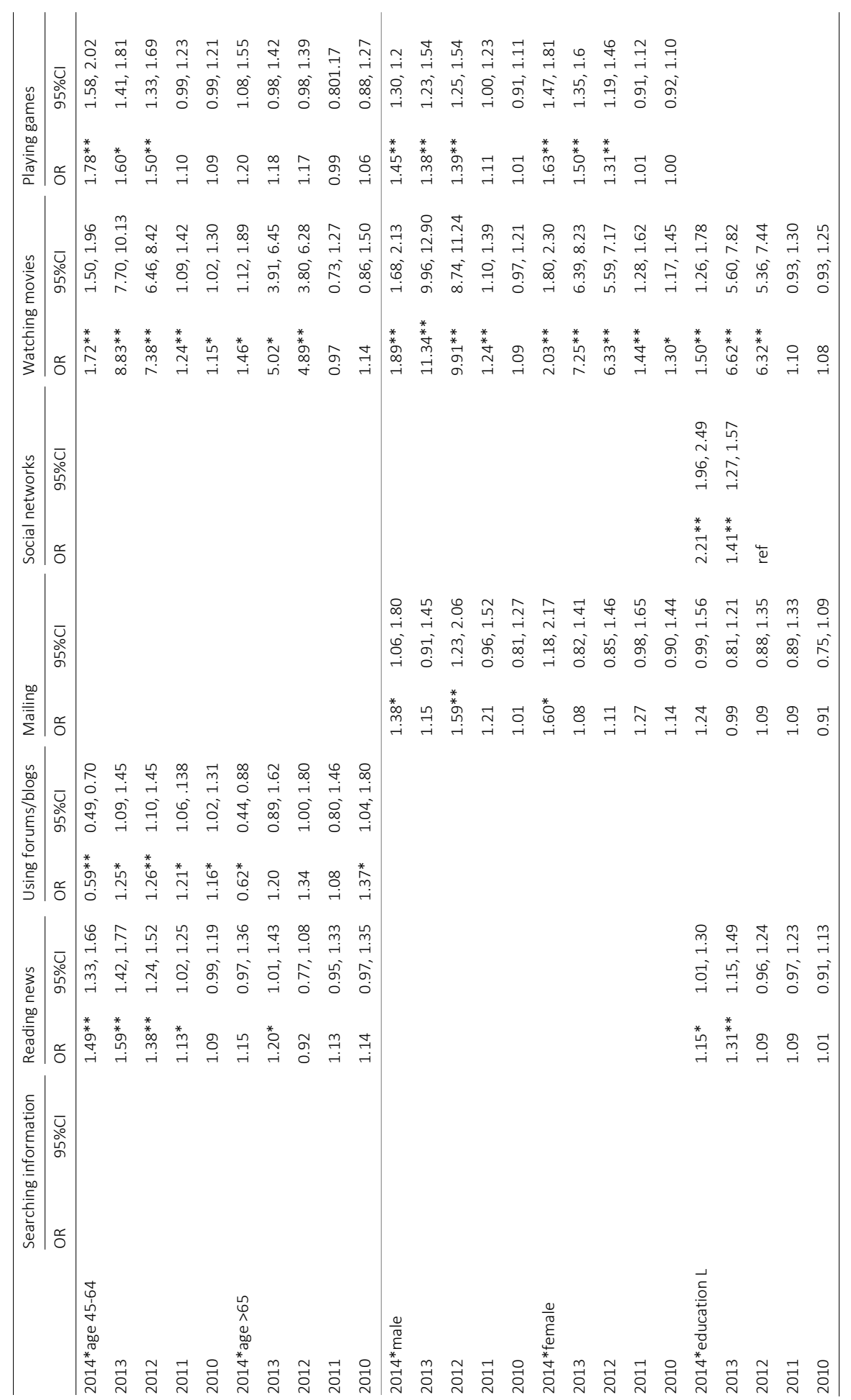




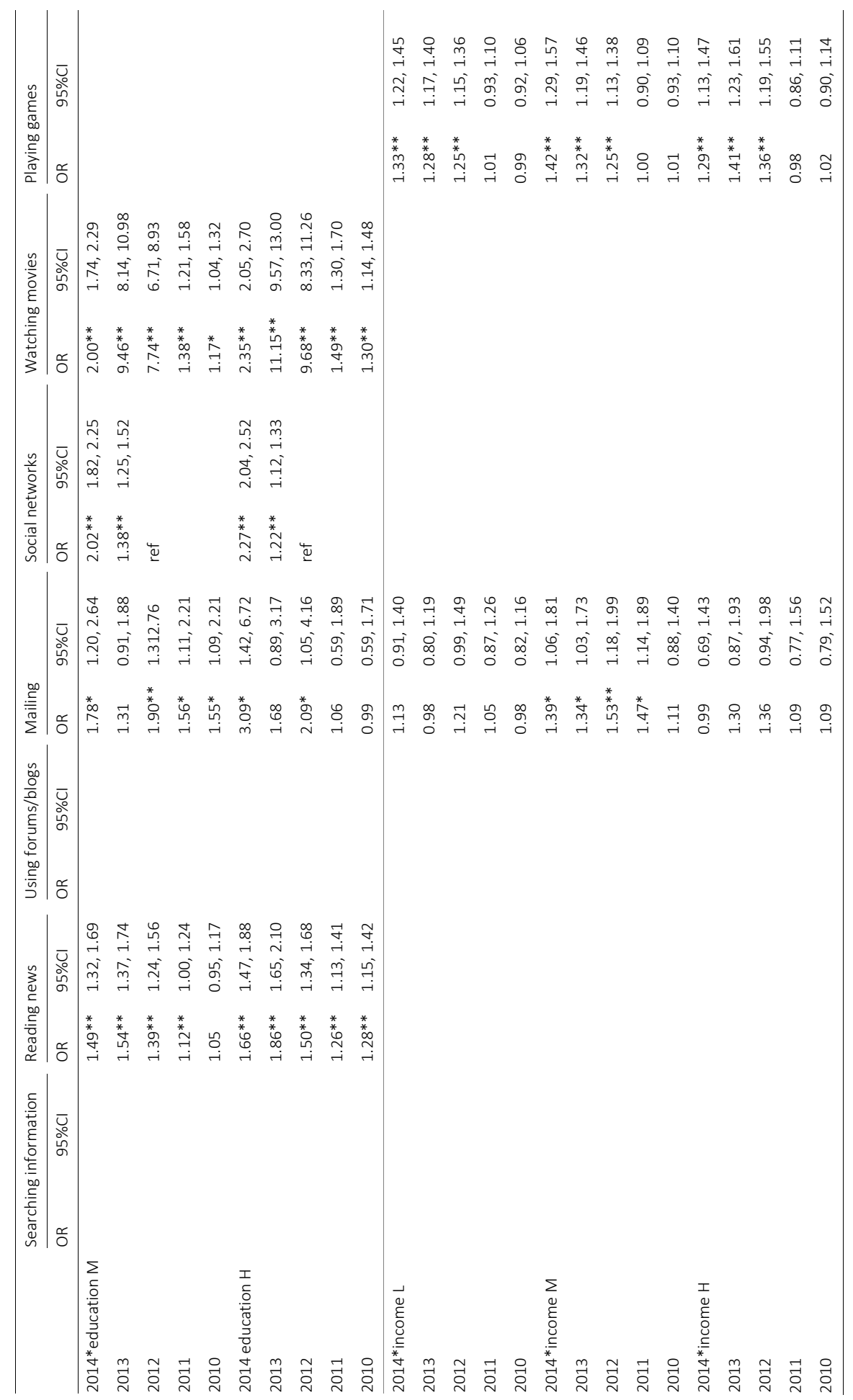


Chapter 2

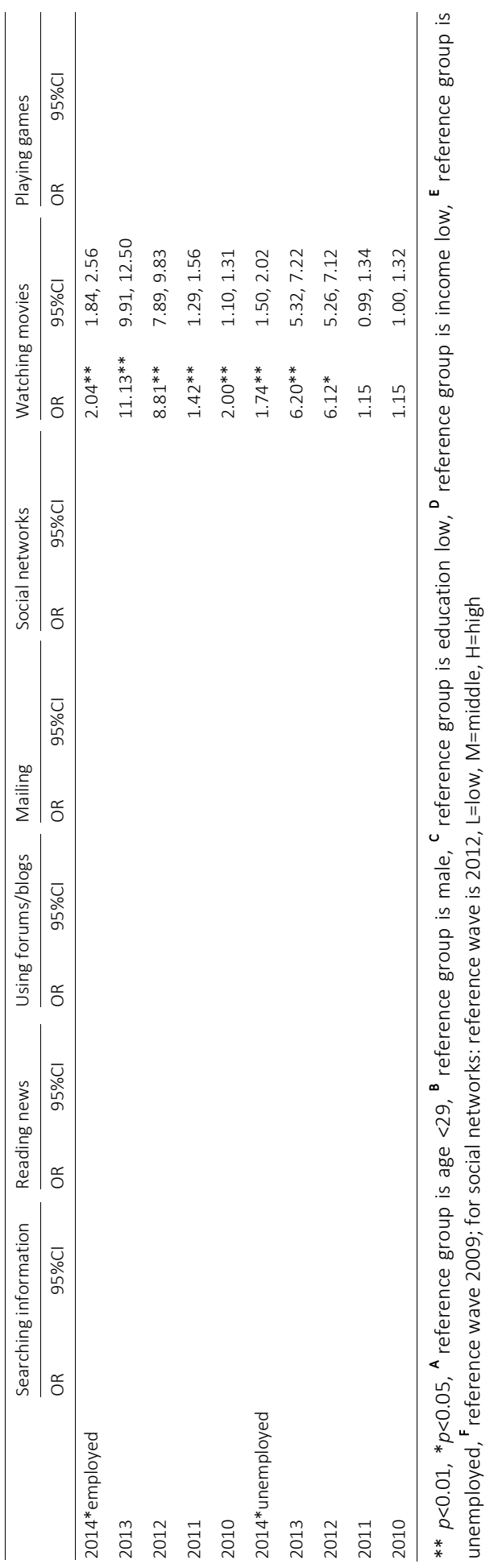




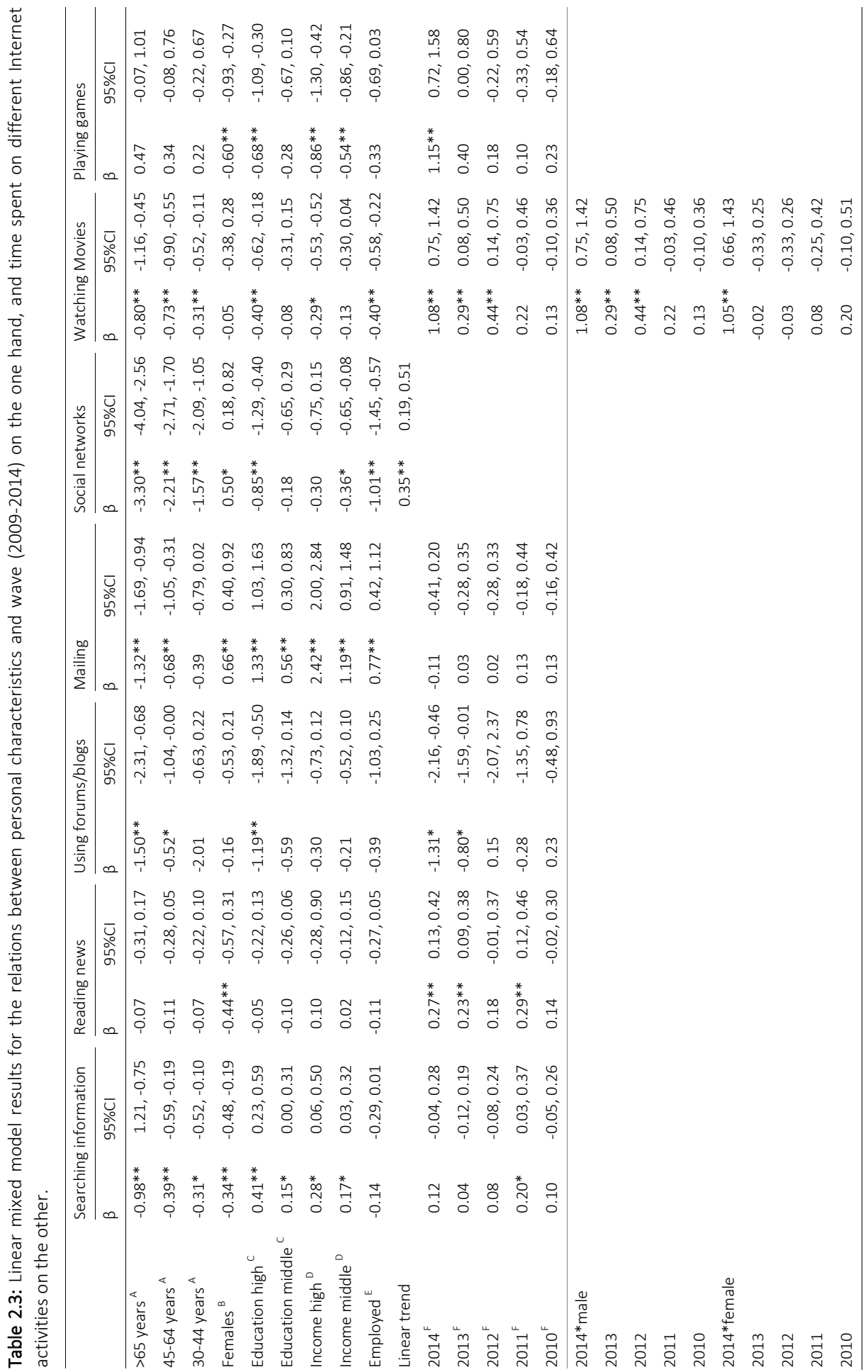


Chapter 2

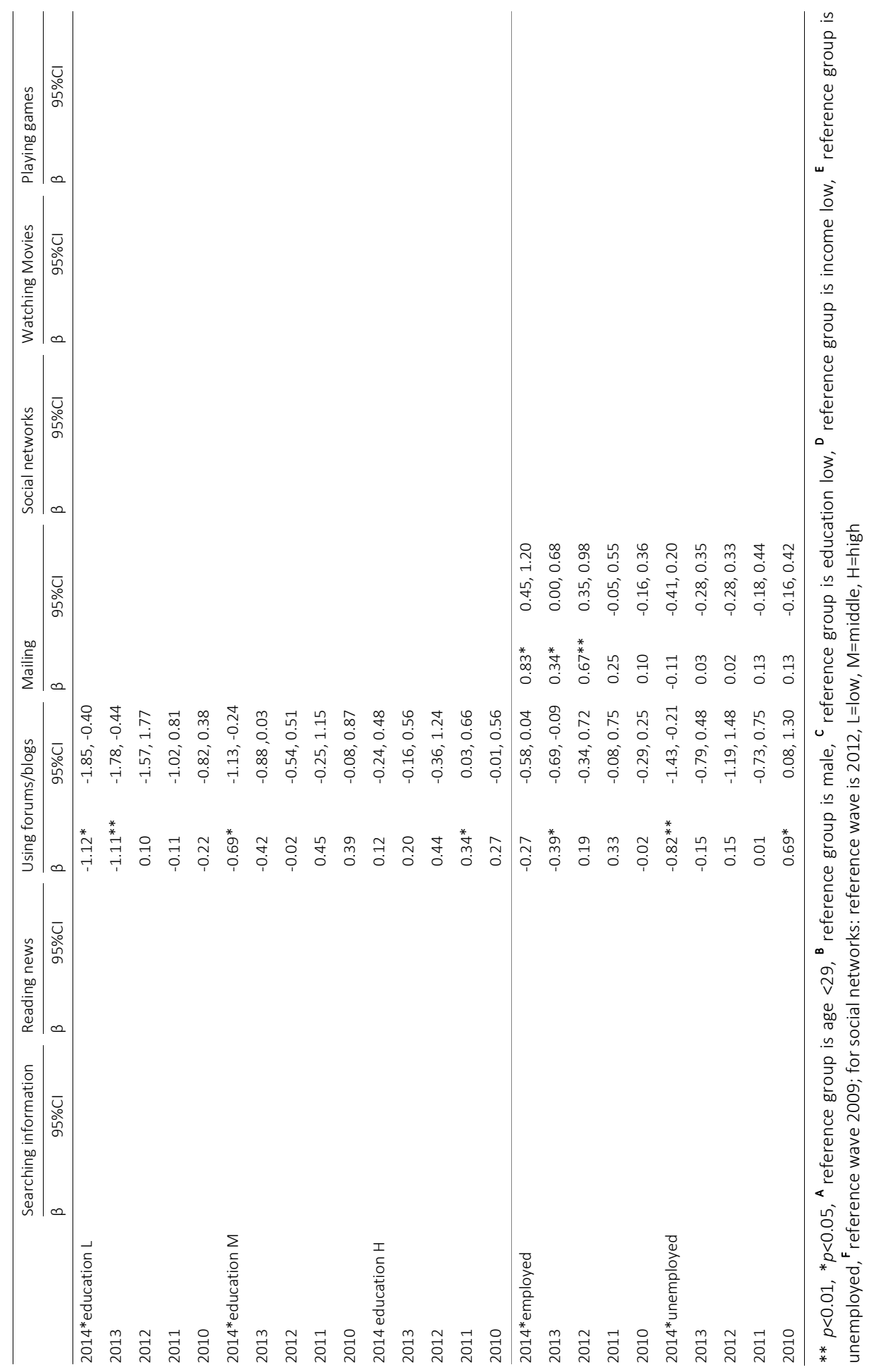




\section{DISCUSSION}

This paper explores the trends in time for seven different online activities between 2009 and 2014 and examines whether these depend on one of five different personal characteristics. Except for being active on forums/blogs, all activities (reading online news, emailing, visiting social networking webpages, watching movies, and playing games online) increased, although with significant differences between subgroups as defined by the five personal characteristics. It was also indicated that some gaps between different groups based on such variables as age or income, in terms of the percentage of persons being online for an activity, have increased. Examples are gaps between age groups with respect to reading news or gaps between income levels with respect to writing emails. Other gaps did not really change such as the one between age groups with respect to searching information.

\section{Age}

In line with previous studies, we found that the older people are, the less likely they are to go online, but in contrast to studies that have found that older people spent more time online reading news and information (Howard et al., 2001; R. B. Jones et al., 2015; S. Jones \& Fox, 2009), we found no such significant difference. An interesting finding is the fact that gaming increases in the older age groups and that they spent similar amount of time gaming compared to younger age groups. Within the LISS questionnaire, they types of games people are playing are not assessed, but it might be reasonable to assume that serious- and social games are played by older people (Information Solutions Group, 2011, 2013), since these games are becoming popular and are perceived as entertaining and promoting social interaction and learning among older adults (A. K. Hall \& Marston, 2021).

\section{Gender}

In line with previous studies, females spend more time engaging in online communication (i.e., emailing) and males are more likely to be active on forums (Boneva et al., 2001; Kalba, 2000; Wasserman \& Richmond-Abbott, 2005; Weiser, 2000). Contrary to other studies, we did not find differences between genders in terms of percentage males and females searching for information, while females spent more time online for this activity. This could be due to the fact that the questionnaire did not distinguish between different types of information. Health seeking, for example, is more common among women (Debrand \& Johnson, 2008; Zillien \& Hargittai, 2009), and while previous studies reported males to be more likely to be engaged in online games (J. Fox 
\& Tang, 2014; Hainey, Connolly, Stansfield, \& Boyle, 2011), our results indicated the opposite. Like the study of van Deursen and van Dijk (2015) our results indicated that slightly more females playing online games and if so, they also spent more time engaging in this activity. A possible explanation for this is that the distinction between different sorts of online games was not made within the questionnaire, and recent statistics show that, for example, puzzle games for mobile phone applications are more popular among females (Lofgren, 2015), while console games are played more by males (Ivory, 2006). It seems possible that the increase in games being played on mobile devices has reduced the gender gap.

\section{Educational level}

In line with previous studies, participants with a higher educational level from our sample used the Internet more often searching for information and spent more time online emailing (Bonfadelli, 2002; CBS, 2014b). Furthermore, our results indicated that participants with a higher educational level more often read news online and also watched more often movies online. It seems reasonable to assume that a higher educational level is related to a work environment that requires email corresponding and the seeking of (work-related) information online. Due to the fact that watching movies is not further defined in the questionnaire, it is also possible that more highly educated people might watch short movies such as tutorials online for work or educational purposes. This might also explain why higher educated people spend less time watching movies.

\section{Income level}

Similar to prior studies, we found that less people with a lower level of income read online news, search for information, and write emails (CBS, 2014b; Madden \& Rainie, 2003). Less frequent people with a lower level of income are active on social networking webpages but people form this income group spent more time for this activity. Furthermore, we did not find that people with a lower income to be more engaged in entertainment activities such as gaming. These findings show that those people who are socioeconomically disadvantaged might make less use of the Internet for personal improvement, such as searching for information or reading news.

\section{Employment}

In line with prior findings that employed people read online news and search for information online more often compared to unemployed people (CBS, 2014b), our results did confirm this. In line with van Deursen and van Dijk (2014), we found that relatively more unemployed people played online games more often. Furthermore, we 
found that a higher percentage of employed people sent emails, however, this difference reached not statistical significance. This might be explained by the fact that employed people send more work-related emails.

These differences in online behavior might suggest causes for concerns because it appears that people with a lower socioeconomic status still make less use of the Internet for personal improvement as indicated by searching for information or reading the news. It seems that the benefits that the Internet offers are sometimes unexploited, which might increase the gaps between socioeconomic groups (Ewa, 2010; Livingstone \& Helsper, 2007). People with a lower socioeconomic status are using the Internet less often for personal development that could be of benefit to them, such as online banking, searching for information regarding political issues, shopping, or traveling (CBS, 2014b). This might also be the case for applications such as eHealth interventions, which might be used less effectively by people with lower socioeconomic status (Bonevski et al., 2014; Eysenbach, 2001).

Most previous studies examined whether people use the Internet or not, and the reasons for any differences in Internet usage range from individual perceptions such as negative attitudes, low self-efficacy, or computer anxiety (Celik \& Yesilyurt, 2013; Charness \& Boot, 2009; Wild et al., 2012). Other studies have examined differences in skills that are related to Internet use, such as Internet literacy and the ability to understand, analyze, evaluate, and produce Internet-related information (Livingstone, 2004; Livingstone, Bober, \& Helsper, 2005). According to van Deursen and van Dijk (2011), skills such as navigation, operational, or information, and strategic skills are also related to Internet skills and are more common among younger people. Furthermore, Internet skills may increase with age and experience (van Deursen \& van Dijk, 2011), but other skills such as information literacy do not change (Hargittai \& Young, 2012). This seems to be problematic because the ability to filter information is not acquired, which might lead to knowledge gaps. These results give reason to recommend more educational interventions in early life (e.g., within school curriculums) to improve Internet skills (van Deursen \& van Dijk, 2009). In addition, interventions for people who left school might be helpful for improving Internet use, such as individualized instructions on how to find information on the Internet for particular purposes. Supporting Internet use has the possibility of increasing Internet self-efficacy by generating positive experiences and could improve Internet use (Eastin \& LaRose, 2000).

\section{CONCLUSION}

From 2009 to 2014, all Internet activities increased, with the exception of being active on forums/blogs. Information seeking and writing emails were the most popular online activity among all subgroups. Activities regarding social interaction (emailing and social 


\section{Chapter 2}

networking websites), entertaining activities (gaming and watching movies), and activities for personal development (reading news and information seeking) increased but differences between subgroups still exist. Further research could aim to determine the reasons for differences in Internet use in order to enable beneficial Internet use interventions for people with different personal characteristics.

\section{Declaration of Conflicting Interests}

Hein de Vries is the scientific director of Vision2Health, a company aimed at implementing evidence based eHealth programs. All other authors declared to have no conflicts of interests.

\section{Funding and acknowledgements}

In this paper we made use of data of the LISS (Longitudinal Internet Studies for the Social sciences) panel. The LISS panel data were collected and administered by CentERdata (Tilburg University, The Netherlands) through its Measurement and Experimentation in the Social Sciences (MESS) project, funded by The Netherlands Organization for Scientific Research. To conduct the present study, the researcher received no specific grant from any funding agency in the public, commercial, or notfor-profit sectors. 


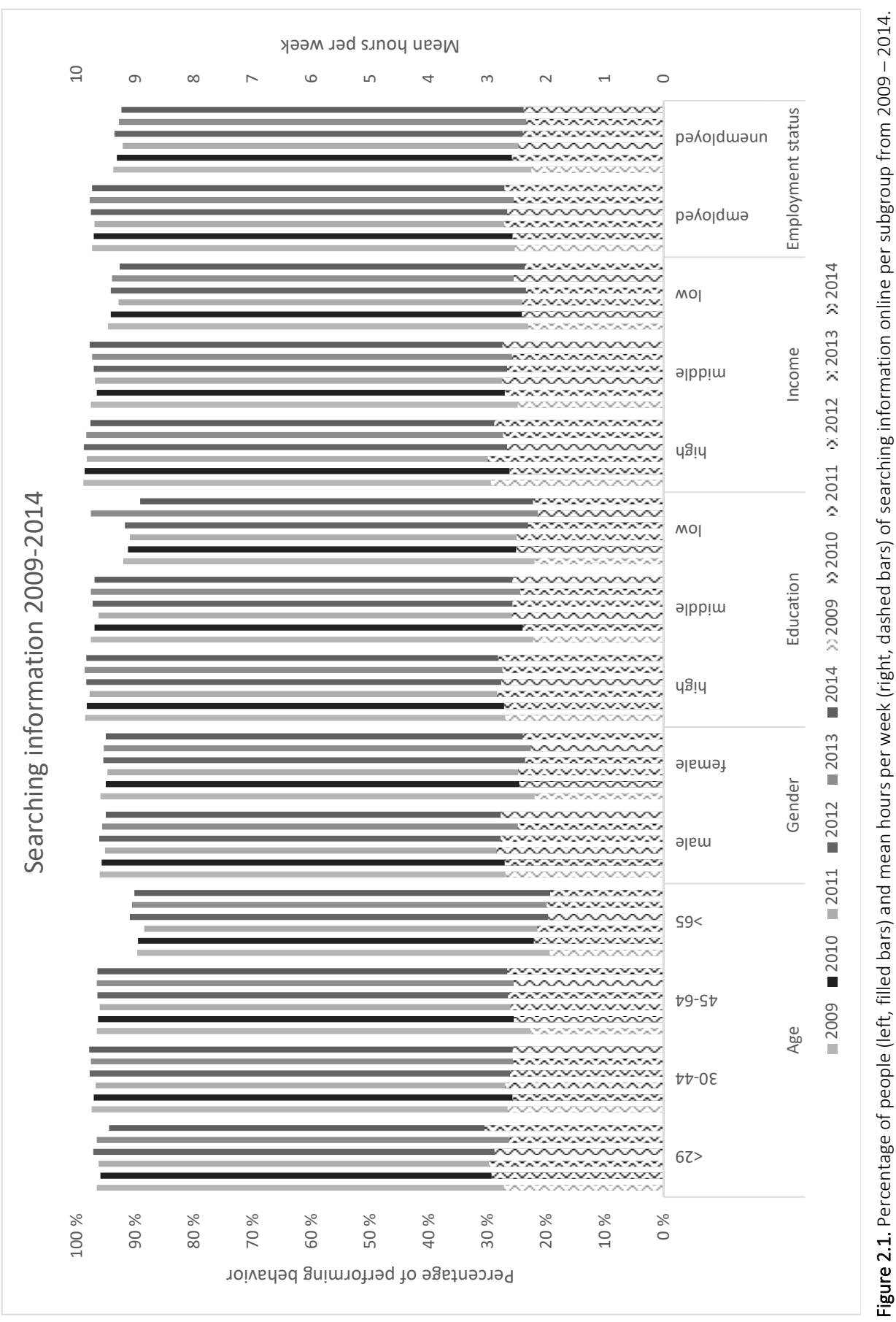




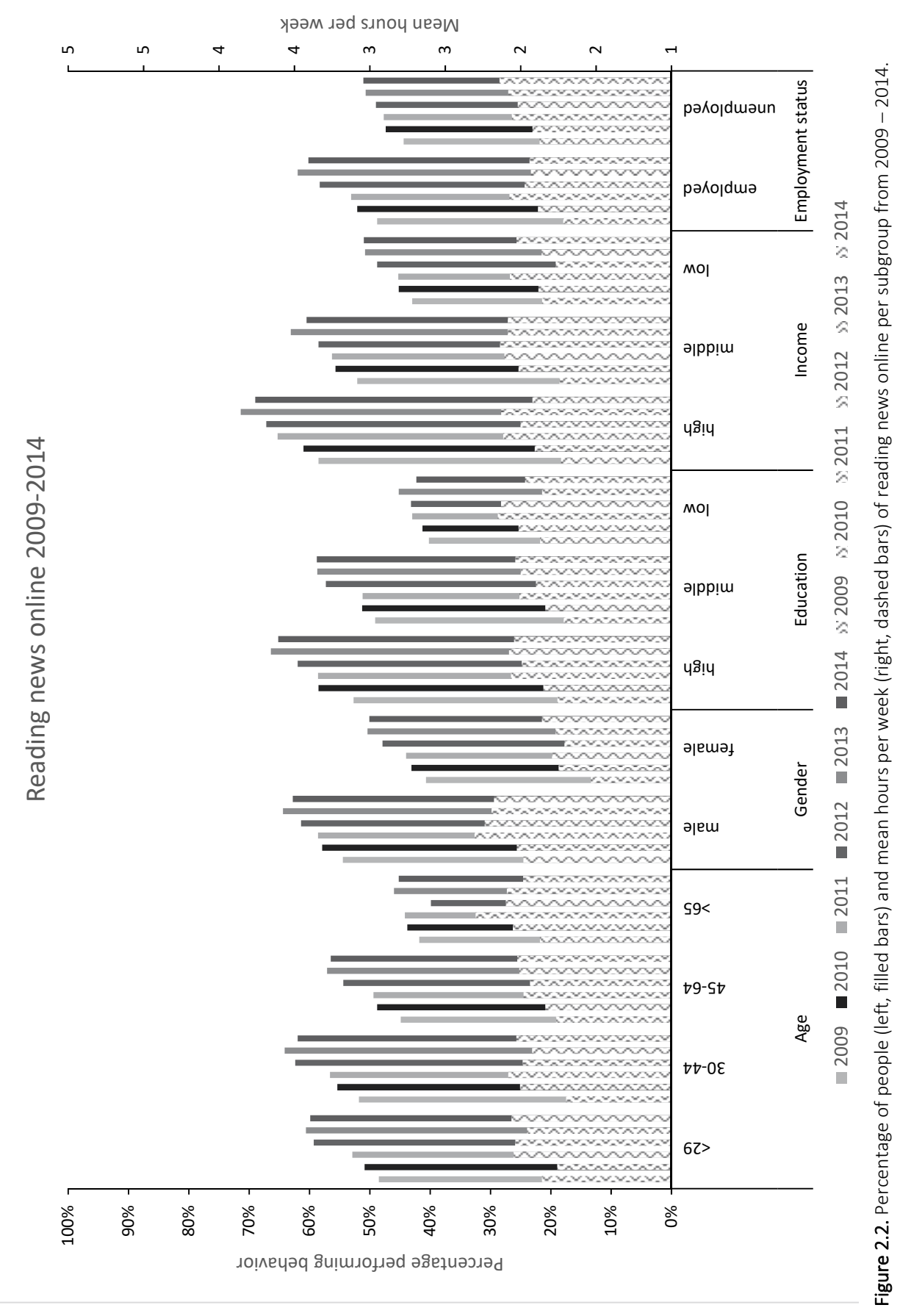




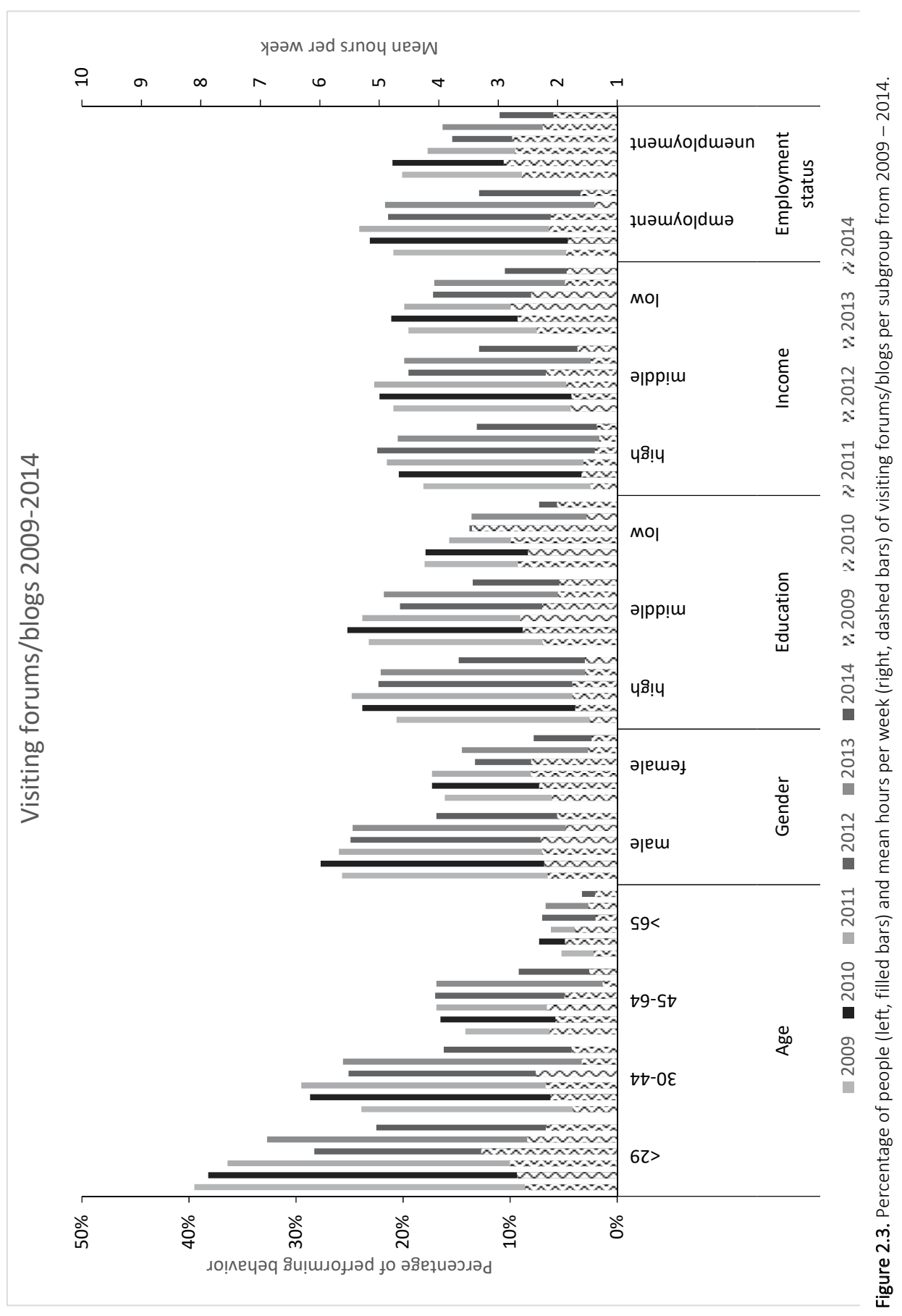




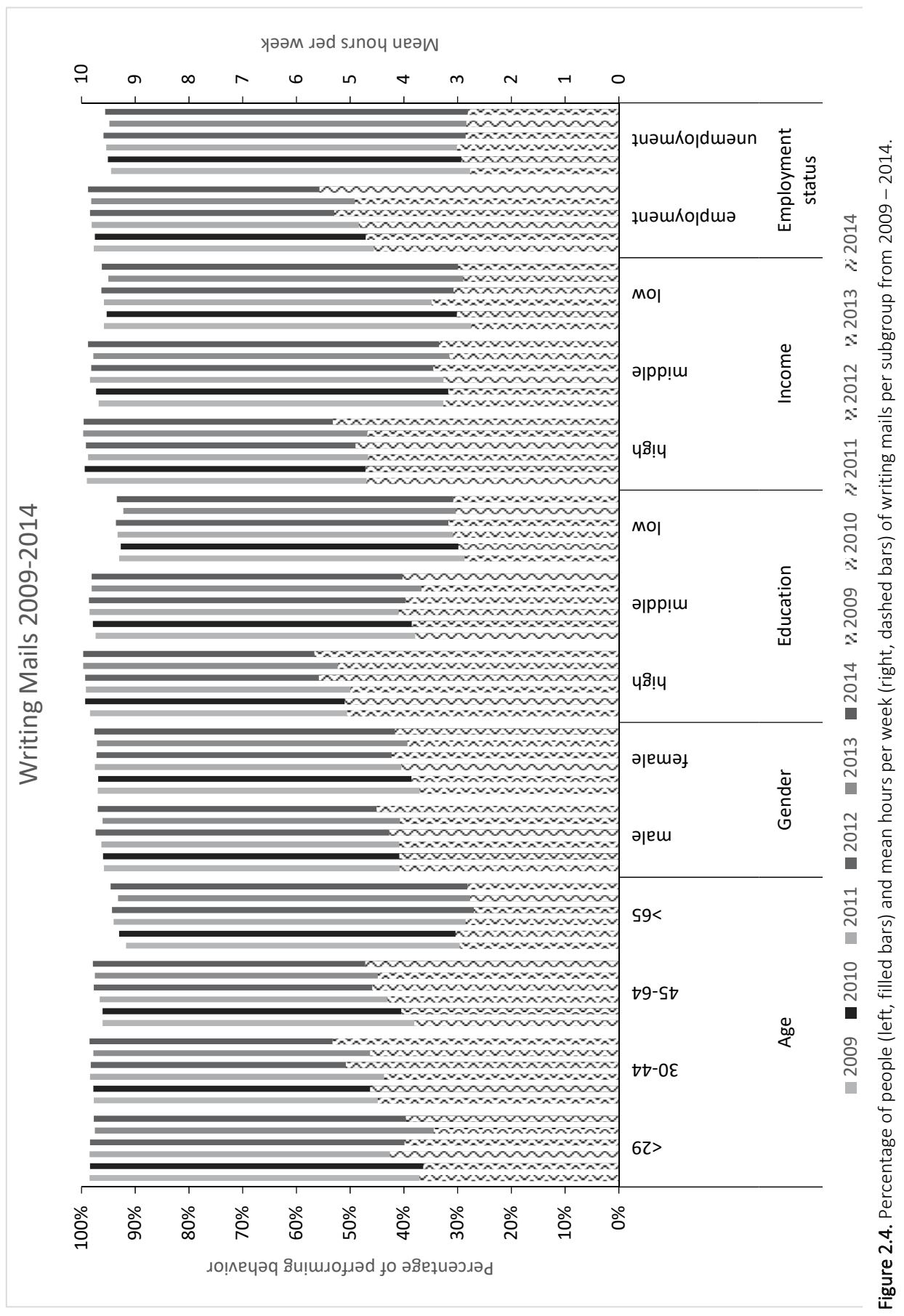




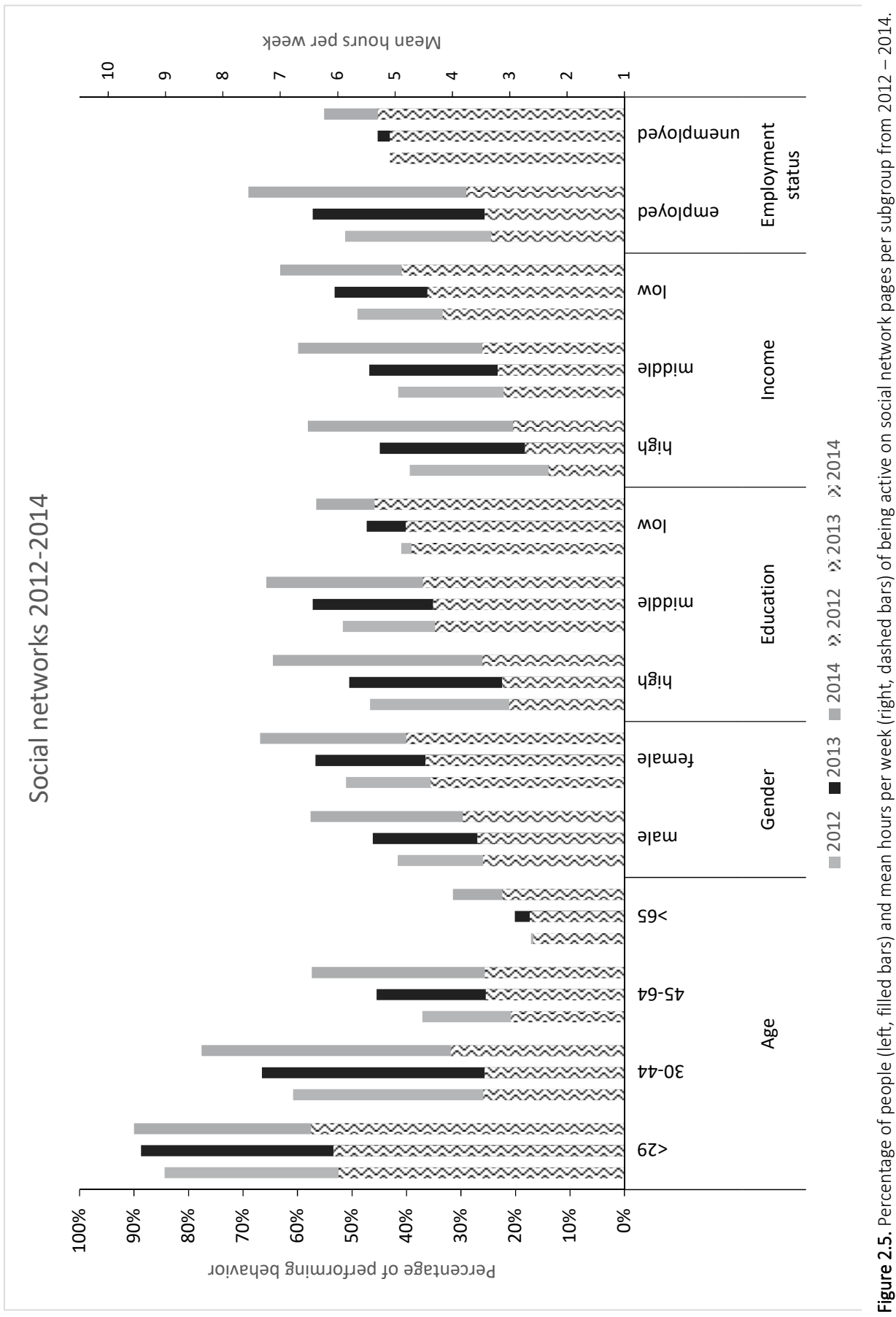




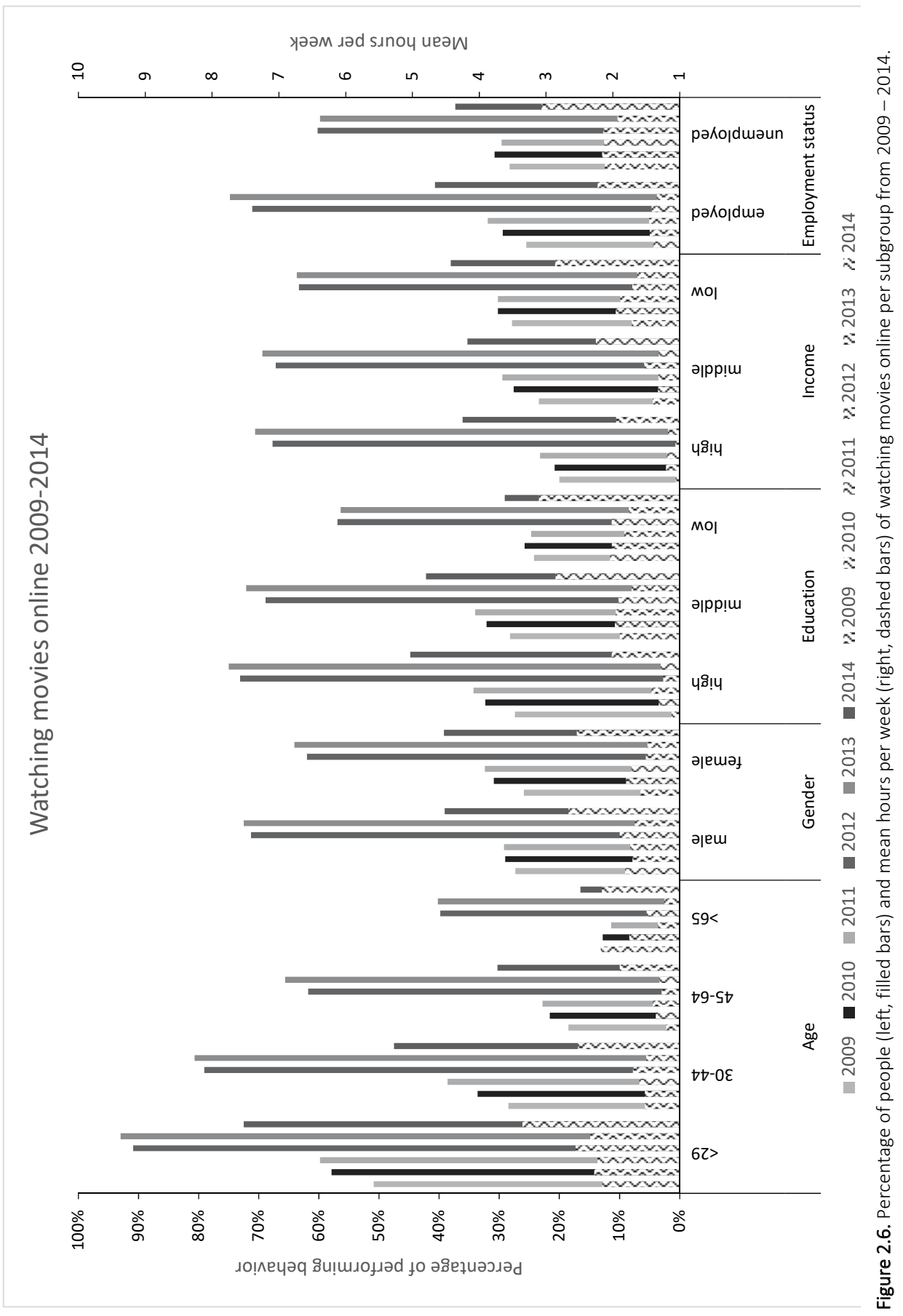




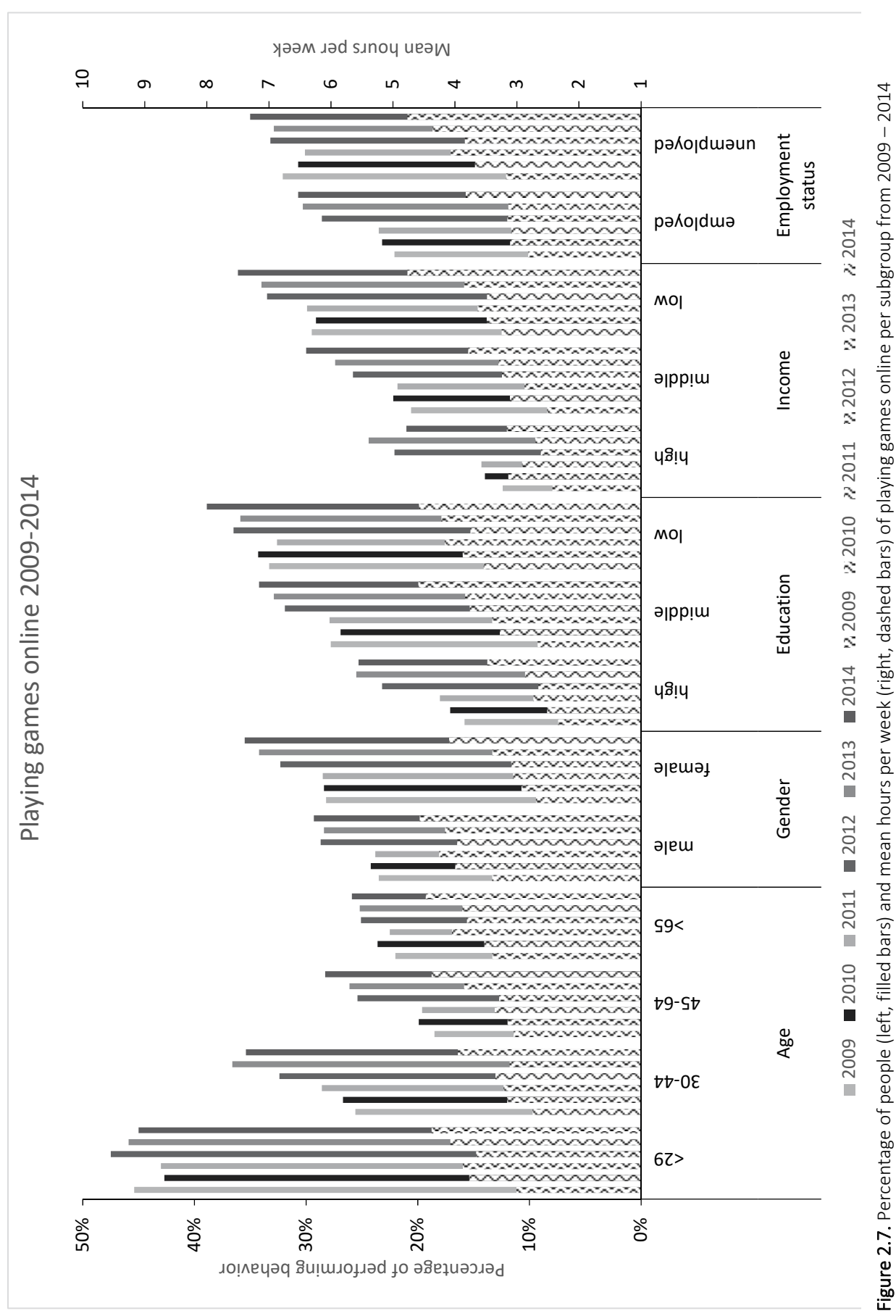





\section{ABSTRACT}

Background: By a legal obligation, the Dutch government publishes online information about tobacco additives (TA) to make sure that it is publicly available. Little is known about the effect of visiting this website (www.tabakinfo.nl) and how it is evaluated by visitors. This study assessed how visitors used the website, its effects on knowledge, risk perception, attitude, smoking behaviour, and how the website was evaluated.

Methods: A randomized controlled trial was conducted: participants were asked to fill out an online questionnaire about TA. Next, participants were randomly allocated to one of the two experimental groups and were invited to visit the website providing information about TA (either with or without a database containing product specific information) or a control group. Follow-up measurement took place after three months. Results: At follow-up ( $n=492)$, no statistical significant differences were found for knowledge, risk perception, attitude, or smoking behaviour in the intervention and control groups. Website visit was positively related to younger participants $(B=-0.07$; $[\mathrm{Cl}=-0.12,-0.01], \mathrm{t}(11)=-2.43, p=.02)$ and having a low risk perception towards TA $(\mathrm{B}=-$ $0.32,[C l=-0.63,-0.02], t(11)=-2.07, p=.04)$; whereas being lower educated $(B=-0.67$, $[\mathrm{Cl}=-1.14,-0.17], \mathrm{t}(11)=-2.65, p=.01)$ is a significant predictor for making less use of the website Furthermore, the website has been evaluated less positive by smokers compared to non-smokers $(\mathrm{t}(324)=-3.55, p \leq .001)$ and males compared to females $(\mathrm{t}(324)=-2.21, p=.017)$.

Conclusion: The website did not change the perceptions of TA nor smoking behaviour. Further research is necessary to find out how effective health communication about the risks of TA can be realised by providing online information.

Trial Register: Nederlands Trial Register NTR4620

Keywords: tobacco additives, information dissemination, website use, website evaluation, RCT 


\section{INTRODUCTION}

Cigarettes and other manufactured tobacco products contain numerous additives (Merckel \& Pragst, 2007). A total of 673 different tobacco additives (TA) are used during the production of cigarettes (Schenk, van de Nobelen, Pennings, Kienhuis, \& Talhout, 2015) and one cigarette contains on average 68 different additive (Talhout, 2012 ). Additives such as sugar or vanillin may be perceived as being harmless, but develop into harmful carcinogen substances (e.g., formaldehyde) during the combustion process (Merckel \& Pragst, 2007; Songur, Ozen, \& Sarsilmaz, 2010; Swenberg et al., 2013). Some TA are thought to cause a higher bioavailability of nicotine, which increases nicotine addiction (Rabinoff, Caskey, Rissling, \& Park, 2007). For the tobacco industry, one of the main reasons to add TA is to improve the taste and to make the smoke milder and consumable (Rabinoff et al., 2007).

Since 2003, tobacco producers and importers in the Netherlands are legally obliged to list all TA used in their products and provide this information to the government (RIVM, 2015). Information about the amount of used additives, the function and known impact on the health must be declared (Kienhuis, Schenk, \& Talhout, 2013). The Dutch National Institute for Public Health and the Environment (RIVM) is publishing this information about TA on a dedicated website (www.tabakinfo.nl) since 2012. The aim of this website is to provide neutral and objective information to the general population. In contrast to the Netherlands, most other European countries that collect information about TA do not publish this information. In Great Britain for example, this information is not published because it is feared that people might misunderstand the information (Talhout, 2012 ).

Only two studies investigated the influence of public dissemination of information about components in tobacco or smoke, in Australia (Ipsos-Eureka, 2009 ) and the United States (M. G. Hall, Ribisl, \& Brewer, 2014). Results from focus group interviews in Australia demonstrated that information about TA is desired by the general public, but they were not interested in looking up this information while they did feel that information about additives might have an influence on smoking behaviour. Moreover, some information appeared to be too detailed or confusing. Additionally, TA information led to some misconceptions. For example, some people thought that cigarettes with fewer additives were less dangerous than cigarettes containing more additives (Ipsos-Eureka, 2009 ). A cross-sectional quantitative survey in the United States on the impact of smoke components information on smokers and non-smokers by assessing awareness, worries and smoking discouragement effects (M. G. Hall et al., 2014). The study participants were aware of six of 20 mentioned components. Reading TA information was associated with increased levels of worry about harmfulness of substances that were added to tobacco products, such as TA, compared to substances that are naturally in cigarette smoke. However, smoke components differ from TA. TA are intentionally added during the manufacturing process of the tobacco product to 
improve taste or product quality, whereas smoke components are the substances to which smokers are exposed during use of the product. Still, the US study can inform on how people perceive information on TA.

It is conceivable that information about TA and smoke components may not be interesting to everyone. Smokers are known to be less interested in information about risks of smoking (Weinstein \& Nicolich, 1993) in comparison with non-smokers. It might also be that people with different educational levels understand the given information about TA and smoke components differently or might even misunderstand them. Previous studies that would underpin this assumption are rarely about TA, but have been conducted for potential reduced exposure products (PREP) (e.g., light cigarettes). Some studies demonstrated that smokers believed that light products were less harmful compared to regular cigarettes (Cummings et al., 2004; Hamilton et al., 2004). Although the given information about the PREP products did not contain any statements about positive health outcomes; smokers thought that these products were healthier compared to regular cigarettes (Hamilton et al., 2004). Furthermore, higher educated people were more aware of those products, but no differences were found with regard to risk perceptions of these products when comparing different educational levels (O'Connor, Hyland, Giovino, Fong, \& Cummings, 2005). Another study about the reaction to reduced risk tobacco advertisement described that people with a lower educational level misinterpret the advertisement and thought those products were complete free of health risks (Shiffman, Pillitteri, Burton, \& Di Marino, 2004). Moreover, higher educated people used PREP more frequently than lower educated people (Parascandola, Augustson, \& Rose, 2009). These results show that information can have a misleading impact and the risk that smokers and non-smokers as well as people with different educational levels could misunderstand the online provided information about TA and smoke components. Given that information on TA is mandatory nowadays, it is important to find out, whether this information may result in changing knowledge, risk perceptions, and attitudes about TA, since these determinants are important in the intention forming in health related behaviours (Fishbein \& Ajzen, 2011; Grube, Morgan, \& McGree, 1986; Tyas \& Pederson, 1998). These insights are currently lacking in the Netherlands.

The first aim of our study is to describ the effects of a randomized controlled trial RCT in terms of knowledge, risk perception, attitude change about TA and smoking behaviour in terms of amount of daily smoked cigarettes. The second aim is to assess the website usage. Finally, we will describe the visitors' evaluation of the website. 


\section{METHOD}

The website www.tabakinfo.nl

For this study, we made a copy from the original website and deactivated hyperlinks to other information than about TA which are also available on the original website. By doing this, we wanted to reduce the attention to other information and being able to measure participants' usage behaviour. On the website www.tabakinfo.nl participants could find information about the legislation about TA, why TA are added and in which products. Furthermore, 15 specific TA are listed in detailed such as sugar or vanillin, in factsheets that provide more information about these additives function, and their potential harms. The website provides also a database where visitors can search for specific products by brand and get an overview about the composition and the additives.

\section{DESIGN}

An RCT with three study groups (Tobacco Info, Tobacco Info +, control group), one baseline and one follow-up measurement was conducted (Figure 3.1). Participants in all three groups had to fill in the baseline questionnaire. Afterwards, the "Tobacco Info" group was asked to visit the website and to read information about TA only. The "Tobacco Info +" group was also asked to visit the database and in addition to look up the information about TA for a cigarette brand and type. This information was not available on the website for participants who were allocated to the "Tobacco Info" group. This manipulation was done in order to investigate the possible effect of visiting the database. The follow-up measurement took place after three months. The control group only had to complete the baseline and follow-up questionnaires. Ethical approval of the Regional Medical Ethics committee in the Netherlands was not necessary, because participants in this study were not subjected to procedures or required to follow certain rules of behaviour, which are the criteria for ethical approval (CCMO, 2015).

\section{Procedure and participants}

Participants older than 18 years were recruited via a Dutch independent Internet panel that operates in line with the ISO standards (Flycatcher Internet Research, 2015). All members in this panel expressed their willingness to participate in scientific research studies. In total 1000 panel members were invited to participate in this study, of which 672 panel members did (response rate of 67\%). Data collection took place in July (baseline) and October (follow-up) 2014. After receiving the email, participants had one week the time to fill in the questionnaire. One email reminder was sent to participant's 
which did not responded after five days. To be able to make a comparison between smokers' and non-smokers' perceptions of the website, we aimed to include $50 \%$ smokers and 50\% non-smokers in the study sample. Panel members received an e-mail with a link to the online questionnaire and at the end of the questionnaire a unique link to the study website was included (for experimental groups). The use of a unique link for each participant provided the opportunity to track participant's behaviour on the website (i.e., which parts of the website the participants visited). The invitation for the follow-up measurement was only send to participants who completed the baseline questionnaire. Participants received incentives in the form of points they can collect and exchange against vouchers.

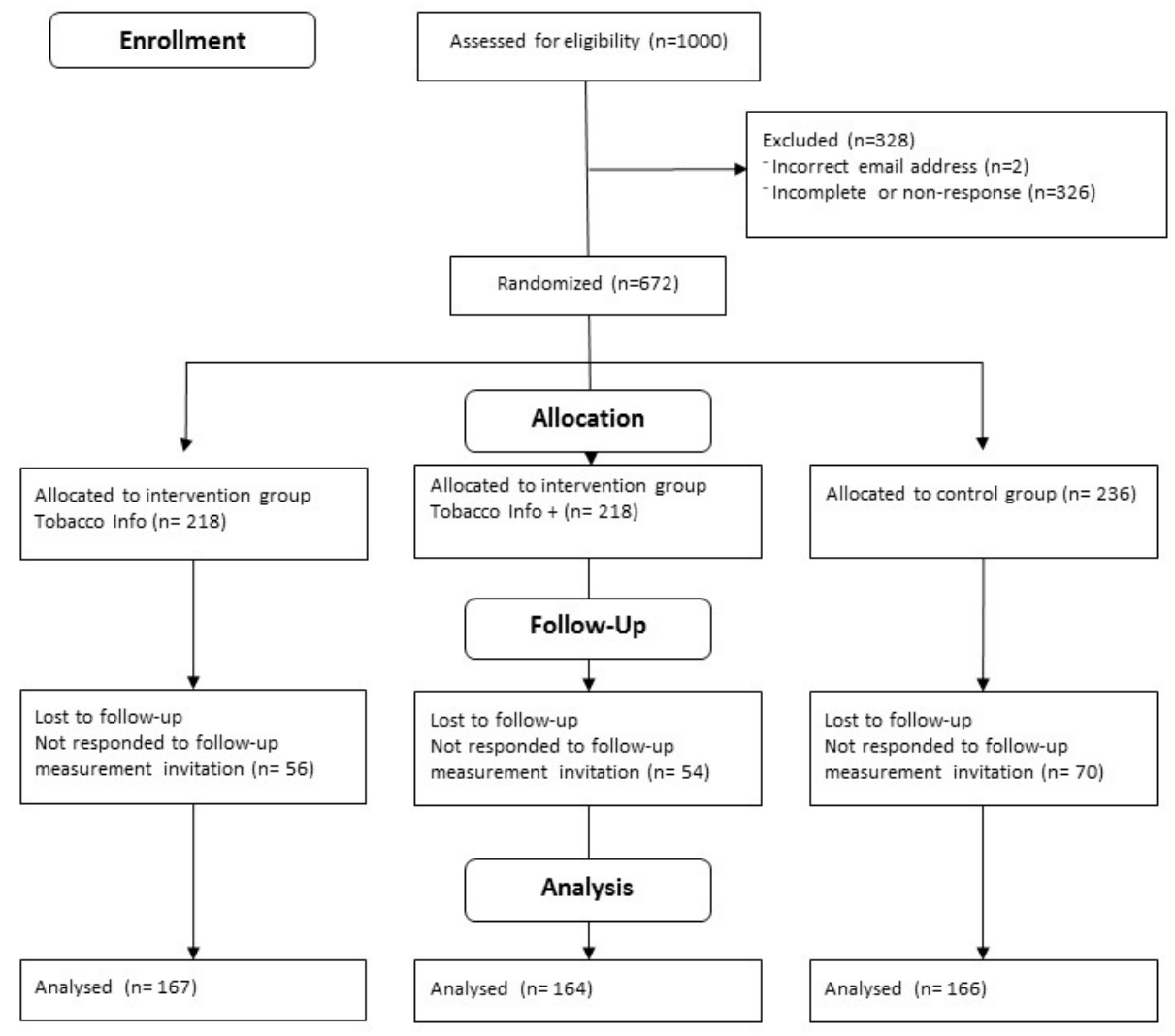

Figure 3.1: Flow chart of study design

\section{Questionnaire}

The questionnaire was administered in Dutch. Personal characteristics were assessed only at baseline and included gender ( $1=$ male, $2=$ female), age (1=18-19, $2=20-24,3=25$ - 
$29,4=30-34,5=35-39,6=40-44,7=45-49,8=50-54,9=55-59,10=60-64,11=65$ years or older), educational status ( $1=$ low: no education, primary or lower vocational school, $2=$ middle: secondary vocational school or high school, $3=$ high: higher professional education or university), and income (1=low: <12.500€; $2=$ middle: $26.000 €-39.000 €$; $3=$ high: $39.000 €$ or more) The distribution of the sample was in line with the national Dutch distribution as described by Statistics Netherlands (www.cbs.nl).

At baseline, participants were asked with one item if they knew the website already: "Are you familiar with the website www.tabakinfo.nl?" (1=yes, 2=no).

Smoking behaviour, knowledge, risk perception, and attitude about TA, were measured at baseline and after three months follow-up. To assess smoking behaviour, all participants were asked whether they smoked at least once a month ( $1=$ yes, $0=$ no). If yes, participants were categorized as smokers and they were asked to indicate with a multiple choice question whether, and how much a day, which brand and type they smoke of cigarettes, cigarillos, cigars, rolling tobacco or pipe.

Omega $(\Omega)$ was used to assess the internal structure of the scales (Peters, 2014); this value represents a less biased alternative to Chronbach's alpha (Dunn, Baguley, \& Brunsden, 2014). Knowledge about TA was assessed with 10 right or wrong statements $(\Omega=0.82,95 \% \mathrm{Cl}=0.80-0.84)$. To dichotomous knowledge we recoded "I don't know" answers into answering this question wrong since the right answer was not known. For example: "Tobacco with additives consists of less carcinogen substances compared to tobacco without additives" (wrong).

To measure risk perception of TA, six items were assessed, three items covering cognitive and three items covering affective aspects of risk perception. These questions were based on earlier questionnaires assessing risk perception and were adjusted for TA (Janssen, van Osch, Lechner, Candel, \& de Vries, 2011). Answers were given on a 5point Likert scale (totally agree $=5$, totally disagree $=1 ; \Omega=0.81,95 \% \mathrm{Cl}=0.77-0.84$ ). "If I smoke tobacco with additives I have a high chance of getting cancer", is an example item of a cognitive risk perception item.

Attitude about TA was assessed by 14 items on a 5-point Likert scale (totally agree=5, totally disagree $=1$ ), eight items about pros regarding TA and six items about cons of TA (pro $\Omega=0.83,95 \% \mathrm{Cl}=0.79-0.86$; con: $\Omega=0.79,95 \% \mathrm{Cl}=0.76-0.82$ ). These questions were based on earlier questionnaire assessing attitude and were adjusted for TA (D. N. Schulz et al., 2011; D. N. Schulz, Kremers, et al., 2014). As an example for a pro attitude item, participants were asked "If I would smoke tobacco with additives, I feel good".

Questionnaire evaluation was included in all studies that are conducted in the panel used in the study at hand. On a visual analogue 5-point Likert-scale participants rated whether they evaluated the questionnaire as interesting (1) or uninteresting (5), pleasurable (1) or not (5), too long (5) or too short (1), and difficult (1) or easy (5) to answer. We also documented how many minutes it took to complete the questionnaire.

The follow-up measurement contained items about the website evaluation (Crutzen, Cyr, \& de Vries, 2011). Participants perception about the website was assessed with 10 
concepts, completeness and layout were assessed with two items, the other concepts with three items each: Efficiency (e.g.: I easily find information I am looking for on this website; $\Omega=0.92,95 \% \mathrm{Cl}=0.90-0.93$ ), effectiveness (e.g.: The website provides useful information; $\Omega=0.92,95 \% \mathrm{Cl}=0.91-0.94$ ), enjoyment (e.g.: I found my visit at this website enjoyable; $\Omega=0.96,95 \% \mathrm{Cl}=0.95-0.96$ ), active trust (e.g.: I would act on the information presented on this website if needed; $\Omega=0.89,95 \% \mathrm{Cl}=0.86-0.91$ ) (Crutzen, Beekers, et al., 2014; Crutzen, Cyr, et al., 2011), relevance (e.g.: The information on the website were new to me; $\Omega=0.78,95 \% \mathrm{Cl}=0.71-0.80$ ), understanding (e.g.: I found many words on the website difficult to understand; $\Omega=0.83,95 \% \mathrm{Cl}=0.82-0.88$ ), completeness (e.g.: The website contains enough information; $r=0.79$ ), layout (e.g.: I found the layout of the website looking good; $r=0.29$ ), recommendation to others (e.g.: I would recommend the website to others; $\Omega=0.83,95 \% \mathrm{Cl}=0.84-0.89$ ) and intention to revisit (e.g.: I would revisit the website again; $\Omega=0.84,95 \% \mathrm{Cl}=0.81-0.87$ ). All items could be answered on a 5 -point Likert scale ( 1 totally disagree until 5 totally agree).

Website use was assessed based on tracking the unique links participants received. Using these unique links, it was able to see which pages each participant visited.

\section{Statistical analyses}

Data were analysed using the software SPSS, version 24 and R was used to calculate omegas as scale reliability. Logistic regression analysis was used in order to evaluate whether dropout was higher among specific subgroups. Frequencies and descriptive statistics were used to describe the study sample at baseline.

Linear regression using enter method was used to analyse differences in knowledge, risk perception, attitude, and the amount of smoked cigarettes per day between control and study groups after visiting the website. All analysis were done for these five outcome variables with age, gender, level of education and income, and smoking status as independent variables. Further, interaction of study group*age, study group*smoking status, and study group*education in order to determine any differences among these subgroups. If one of these interaction terms were significant at a $p$-value of $\leq .05$, analyses for the relevant subgroups were done. All analysis was corrected for age, gender, educational level, income, and smoking status. Analysing follow-up effects of knowledge, risk perception, attitude, or smoking, the corresponding baseline measurement was considered.

Linear regression analyses using enter method were conducted to determine predictors of website use. For this purpose a continuous dependent variable was calculated which sums up the numbers of visited pages within the website which includes TA specific information. Independent variables were age, gender, education, income, knowledge, attitude, risk perception, smoking status, amount of smoked cigarettes, duration of questionnaire, and questionnaire evaluation. Participants who reported at baseline to know the website $(n=19)$ were excluded for the analysis. 
Finally, we analysed if the website was evaluated differently by different subgroups. For this purpose we conducted independent samples t-test to compare males with females, and smokers with non-smokers, with ANOVA Tukey post hoc tests we compared educational and income levels and used the means of each of the ten evaluation concepts (efficiency, effectiveness, active trust, relevance, enjoyment, understanding, completeness, layout, recommendation to others, intention to revisit).

\section{RESULTS}

Sample characteristics and attrition

The study sample (Table 3.1) consisted of 672 participants at baseline $(54.8 \%$ male, $53.9 \%$ smoker). In terms of gender, age, the distribution within the study sample is in line with the distribution in the Netherlands as well as the distribution of income $131.7 \%$ high, $45.2 \%$ middle, $23.1 \%$ low), and education (26.6\% high, $43.2 \%$ middle, 32.3\% low).

The loss to follow up after three months was 26.8\% (180/672). Dropout was significantly higher for participants with a lower income (OR 0.42; 95\% Cl=0.19-0.90; $p=.026)$, higher within the experimental groups $(\mathrm{OR}=2.17 ; 95 \% \mathrm{Cl}=1.12-4.22 ; p=.023)$, and among participants who indicated at baseline that they did not liked to fill in the questionnaire $(\mathrm{OR}=0.48 ; 95 \% \mathrm{Cl}=0.30-0.77 ; p=.002)$. With regard to all other variables (age, gender, smoking behaviour, educational level, evaluation of the questionnaire [clear $(M=1.9, S D=1.08)$, interesting $(M=2.1, S D=1.18)$, length $(M=3.0, S D=0.56)$, pleasurable $(M=2.1, S D=1.06)]$, knowledge, attitude, and risk perception) no significant differences in dropout were found. 
Chapter 3

Table 3.1: Sample characteristics and baseline descriptions about TA

\begin{tabular}{|c|c|c|}
\hline & $\mathrm{n}(\%)$ & M (SD, range) \\
\hline \multicolumn{3}{|l|}{ Age } \\
\hline $18-19$ & $23(3.4)$ & \\
\hline $20-24$ & $58(8.6)$ & \\
\hline $25-29$ & $61(9.1)$ & \\
\hline $30-34$ & $50(7.4)$ & \\
\hline $35-39$ & $47(7.0)$ & \\
\hline $40-44$ & $60(8.9)$ & \\
\hline $45-49$ & $69(10.3)$ & \\
\hline $50-54$ & $66(9.8)$ & \\
\hline $55-59$ & $61(9.1)$ & \\
\hline $60-64$ & $50(7.4)$ & \\
\hline $65+$ & $127(18.9)$ & \\
\hline \multicolumn{3}{|l|}{ Gender } \\
\hline Male & $368(54.8)$ & \\
\hline Female & $304(45.2)$ & \\
\hline \multicolumn{3}{|l|}{ Smoking } \\
\hline Smoker & $362(53.9)$ & \\
\hline Non-smoker & $310(46.1)$ & \\
\hline \multicolumn{3}{|l|}{ Education } \\
\hline Low & $217(32.3)$ & \\
\hline Middle & $290(43.2)$ & \\
\hline High & $165(24.6)$ & \\
\hline \multicolumn{3}{|l|}{ Income } \\
\hline Low & $155(23.1)$ & \\
\hline Middle & $304(45.2)$ & \\
\hline High & $213(31.7)$ & \\
\hline \multicolumn{3}{|c|}{ Baseline perceptions about TA } \\
\hline Knowledge & & $0.42(0.28,0-1)$ \\
\hline Risk perception & & $3.42(0.61,1-5)$ \\
\hline Attitude pro & & $2.59(0.60,1-5)$ \\
\hline Attitude con & & $3.44(0.62,1-5)$ \\
\hline
\end{tabular}

\section{Determinants and smoking status}

As can been seen in Table 3.2, after three month follow up, neither one of the measured concepts with regard to TA (knowledge, risk perception, attitude) nor the amount of smoked cigarettes per day have been changed within the intervention groups in comparison to the control group. Furthermore, smokers had a significant 
lower risk perception and less negative attitudes towards TA than non-smokers. None of the interaction terms are significant which indicated no subgroup effects for smokers, people with different educational levels, and age groups.

\section{Website use}

In total, 341 participants visited the website and specific information about TA were looked up by 129 participants (37\%). Only a small minority of those participants browsed the website to gain more information about one of the 14 specific TA such as vanilla $(n=23,6 \%)$ or sugar $(n=25,7 \%)$. A small proportion of participants from the "Tobacco Info+" group visited the database ( $n=53,30 \%)$.

Table 3.3 shows that being younger $(B=-.07, t(11)=-2.43, p=.015)$ and having a low risk perception towards $\mathrm{TA}(\mathrm{B}=-.32, \mathrm{t}(11)=-2.07, p=.039)$ are significant predictors for website usage. Whereas being lower educated $(B=-.67, t(11)=-2.65, p=.01)$ is a significant predictor for making less usage of the website. 


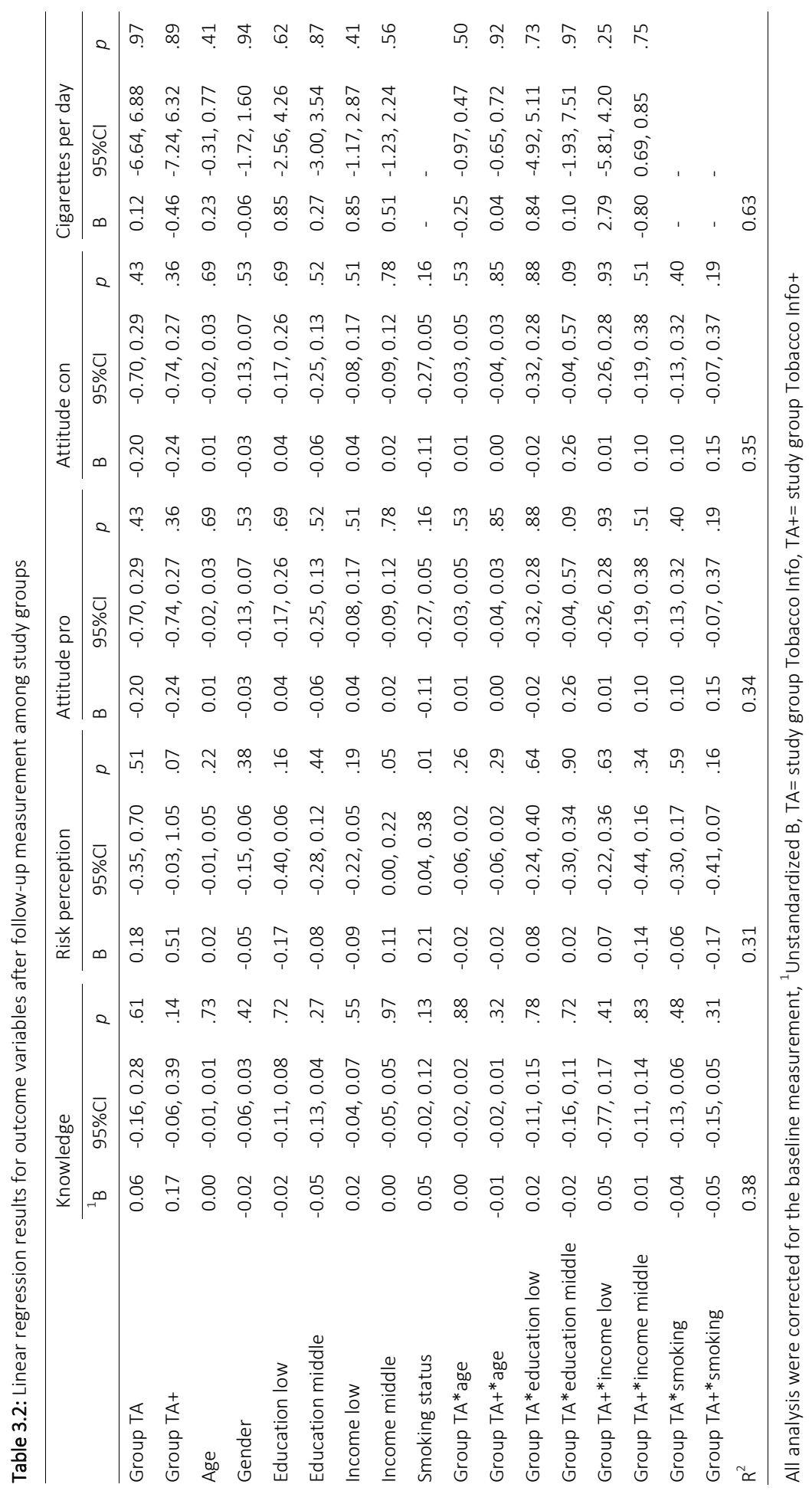


Table 3.3: Linear regression analysis of predictors of website use $(n=337)$

\begin{tabular}{lccc}
\hline Predictor & ${ }^{1} \mathrm{~B}$ & $95 \% \mathrm{Cl}$ & $p$ \\
\hline Smoking & -.08 & $-0.43,0.27$ & .66 \\
Age & -.07 & $-0.12,-0.01$ & .02 \\
Gender & -.15 & $-0.48,0.18$ & .37 \\
Education low & -.66 & $-1.14,-0.17$ & .01 \\
Education middle & -.32 & $-0.75,0,11$ & .14 \\
Income low & -.03 & $-0.51,0.44$ & .89 \\
Income middle & -.10 & $-0.49,0.29$ & .61 \\
Knowledge & .43 & $-0.21,1.07$ & .19 \\
Risk perception & -.32 & $-0.63,-0.02$ & .04 \\
Attitude pro & -.05 & $-0.39,0.28$ & .75 \\
Attitude con & .10 & $-0.25,0.46$ & .57 \\
$R^{2}$ & 0.08 & & \\
\hline
\end{tabular}

${ }^{1}$ Unstandardized B

\section{Website evaluation}

The website was evaluated with the use of the following ten concepts: efficiency, effectiveness, active trust, relevance, enjoyment, understanding, completeness, layout, recommendation to others, and intention to revisit. Table 3.4 shows the mean scores of these scales for the study sample. As can be seen, participants evaluated all these concepts negatively.

For none of these concepts we have found significant differences between participants with different educational levels, income groups or different ages.

Females $(M=2.70, S D=0.73)$ were found to have a significantly higher active trust in the website compared to males $(M=2.53, S D=0.65 ; t(324)=-2.21, M$ difference $=-.02$, $p=.03 ; 95 \% \mathrm{Cl}=-0.33,-0,17)$.

Furthermore, smokers $(M=2.66, S D=0.79)$ compared to non-smoker $(M=2.90$, $S D=0.80)$ had a significantly lower intention to revisit the website again $(t(324)=-3.55, M$ difference $=-0.32, p \leq .001,95 \% \mathrm{Cl}=-0.49,-0.14)$. 
Table 3.4: Website evaluation

\begin{tabular}{ll}
\hline Evaluation & M (SD) \\
\hline Enjoyment & $2.80(0.71)$ \\
Layout & $2.63(0.61)$ \\
Intention to revisit & $2.81(0.81)$ \\
Active trust & $2.61(0.69)$ \\
Recommending to others & $2.65(0.73)$ \\
Relevance & $2.43(0.63)$ \\
Completeness & $2.39(0.66)$ \\
Efficiency & $2.34(0.65)$ \\
Effectiveness & $2.30(0.64)$ \\
Understanding & $2.29(0.66)$ \\
\hline
\end{tabular}

\section{DISCUSSION}

This study showed that participants at baseline did not have a high level of knowledge about TA and no strong positive nor negative risk perception or attitude regarding TA.

We demonstrated that visiting the website resulted in no changes between the control group and the intervention groups at the three month follow-up period. This could be explained by several factors. First, smoking has become a sociable less acceptable behaviour. Therefore, it could be assumed that participants in our study, regardless of the study condition, gave social desirable answers, already at baseline, which could have biased our results in terms of similar answers among the groups (Krumpal, 2013). It might also be possible that completing the questionnaire had an effect on participants from the control group, which is known as mere-measurement phenomenon (Rodrigues, O'Brien, French, Glidewell, \& Sniehotta, 2015; Sprott et al., 2006). In addition, it might also be possible that participants from the control group became curious about the topic of TA and searched for more information about this topic (e.g., using Google). Those participants might have visited the original website and received the same information about TA as the intervention groups of found other information about TA on the web.

Next, with regard to predictors of website use, we found that personal characteristics such as an older age, higher educational level and having a high risk perception towards TA is associated with visiting more sub-websites. This is in line with previous findings that people with a higher socio-economic status are more interested in health related topics and search the Internet more frequently for health information (Mesch, Mano, \& Tsamir, 2012; van Deursen \& van Dijk, 2014). Besides, participants with a low risk perception had visited the website in more detail, supporting earlier findings indicating that high risk perception is associated with avoiding information seeking (Hammond, 2011; Witte, 1994). 
In general, the overall results show that only a minority had visited the website in detail and that the evaluation of the website was not positive. It is important to mention that smokers had a lower intention to revisit the website again but especially those people should be targeted to get informed about tobacco, and the risk of smoking TA. There may be several reasons why people do not visit a website in depth; the surface, design, structure, and function of a website are essential elements which determines how a website will be perceived and used (Garrett, 2010). The evaluation of the website indicates important concepts of the website which should be improved such as understanding, relevance, trust or enjoyment. Improving those websites might motivate visitors to stay longer on the website which in turn increases the change that visitors receiving information about TA. The navigation might be presented clearly without hyperlinks in a text. The content of the information might be more general and less specific since the minority of the participants visited this information. It might be also helpful to guide visitor through the website by options like "which information are you looking for". All these suggestions must examine in further studies.

\section{LIMITATIONS}

There are some limitations to this study. Our participants were recruited via a panel and received a reward for participating. The given information on the website might have a greater impact on people who visit the website pro-actively. Yet, inclusion of visitors only could have jeopardized the generalizability of our findings to the overall Dutch population.

Our model of predicted website use had a very low explained variance (8\%) which indicates that there must be other variables, which we did not measured, related to website use. It is conceivable that there are TA topic related variables which we did not assess such as interest or variables which are associated with Internet usage such health literacy (Diviani, van den Putte, Giani, \& van Weert, 2015).

Finally, we did not find any statistical relevant differences between the control group and the experimental group. Although we cannot preclude the possibility that participants from the control group visited the website between the baseline and the follow-up measurement because the website is easy to find on the web, the findings may also suggest that merely visiting the website once will be insufficient to cause major changes in knowledge, risk perceptions, attitudes, and smoking behaviour. It might be also possible that the website was not encouraging participants to look up information in detail but this need to be evaluated in further studies. 


\section{CONCLUSION}

With the website www.tabakinfo.nl and the disclosure of information about TA, the Dutch government fulfils their requirements to inform the general population (WHO, 2012). Visiting the website did not influence knowledge, risk perception, attitudes towards TA nor smoking behaviour. If website-based health related information will have an impact on concepts such as knowledge, risk perception or attitude it may be relevant to adapt the website more to the needs of the visitors. That could be achieved for example by making the information easily accessible on the homepage and avoid long browsing activities since the most participants only visited the first sub-website. Furthermore, it may be desirable to make the website more attractive to subgroups like less educated people, and smokers. Additionally, as needs of visitors may differ; tailoring the information to the needs is recommended and further research is needed to gain a deeper insight in these needs and requirements.

\section{AUTHORS CONTRIBUTION}

All authors contributed to this article. DR has conducted the overall analyses and drafted the article. HdV and RC contributed to the design of the study. All authors contributed to the interpretation of the data and to the writing of the article. All authors revised the manuscript critically for important intellectual content and read and approved the final manuscript.

\section{FUNDING}

This work was supported by the Dutch Ministry of Health, Welfare and Sport.

\section{CONFLICT OF INTEREST}

AK and RT are working at the RIVM, which is responsible for the content of the website. The study design, questionnaire development, participant recruitment and data analysis were performed independently of the RIVM. HdV is the scientific director of Vision2Health, a company that licenses evidence-based innovative computer-tailored health communication tools. DR and RC declare that they have no competing interests. 


\section{ABSTRACT}

Background: Computer-tailored eHealth interventions to improve health behavior have been demonstrated to be effective and cost-effective if they are used as recommended. However, different subgroups may use the Internet differently, which might also affect intervention use and effectiveness. To date, there is little research available depicting whether adherence to intervention recommendations differs according to personal characteristics. Objective: The aim was to assess which personal characteristics are associated with using an eHealth intervention as recommended.

Methods: A randomized controlled trial was conducted among a sample of the adult Dutch population $(\mathrm{N}=1638)$ testing an intervention aimed at improving five healthy lifestyle behaviors: increasing fruit and vegetable consumption, increasing physical activity, reducing alcohol intake, and promoting smoking cessation. Participants were asked to participate in those specific online modules for which they did not meet the national guideline(s) for the respective behavior(s). Participants who started with fewer than the recommended number of modules of the intervention were defined as users who did not follow the intervention recommendation.

Results: The fewer modules recommended to participants, the better participants adhered to the intervention modules. Following the intervention recommendation increased when participants were older $\left(\chi^{2} 1=39.8, p<.001\right)$, female $\left(\chi^{2} 1=15.8\right.$, $p<.001)$, unemployed $\left(\chi^{2} 1=7.9, p=.003\right)$, ill $\left(\chi^{2} 1=4.5, p=.02\right)$, or in a relationship $\left(\chi^{2}\right.$ $1=7.8, p=.003)$. No significant relevant differences were found between groups with different levels of education, incomes, or quality of life.

Conclusion: Our findings indicate that eHealth interventions were used differently by subgroups. The more frequent as-recommended intervention use by unemployed, older, and ill participants may be an indication that these eHealth interventions are attractive to people with a greater need for health care information. Further research is necessary to make intervention use more attractive for people with unhealthy lifestyle patterns.

Trial Register: Nederlands Trial Register: NTR 2168

Keywords: eHealth; Web-based intervention; intervention use; computer tailoring; personal characteristics; health lifestyle; multiple health behaviors; intervention adherence; socioeconomic status 


\section{INTRODUCTION}

New eHealth interventions are an important tool to improve public health by providing people with information, skills, and support needed for a positive health-related lifestyle change (S. Noar \& Harrington, 2012; D. N. Schulz, Kremers, et al., 2014). These eHealth interventions provide the opportunity to use computer tailoring to provide highly personalized information to a respondent without face-to-face counseling (Brug, Steenhuis, van Assema, \& de Vries, 1996; Krebs et al., 2010; Kreuter, Farrell, Olevitch, \& Brennan, 2000).

With the use of computer tailoring, participants receive information derived from an individual assessment, attuned to their individual answers, and, in our case, aimed at motivating individuals to adopt one or more healthy behaviors (de Vries \& Brug, 1999). Consequently, computer-tailored interventions provide feedback that is more relevant to the individual, contains less redundant information, and is more likely to be processed and remembered than generic information (Brug et al., 2003; Hawkins et al., 2008; Lustria et al., 2013; S. Noar et al., 2007). Furthermore, eHealth interventions are easily accessible and have the potential to reach a wide population (Bennett \& Glasgow, 2009; Griffiths et al., 2006).

Numerous studies have demonstrated that computer-tailored interventions are effective in motivating individuals to adopt health behaviors (Kohl et al., 2013; Krebs et al., 2010; S. Noar \& Harrington, 2012; S. M. Noar et al., 2011), including increased physical activity (Ammann et al., 2013; Kroeze et al., 2006; G. J. Norman et al., 2007; van 't Riet et al., 2010; van Stralen et al., 2011), healthy nutrition (Broekhuizen et al., 2012; Brug et al., 1999; Hamel \& Robbins, 2013; Krebs et al., 2010; Kroeze et al., 2006; Neville et al., 2009), smoking prevention and cessation (de Jong de Josselin et al., 2014; Elfeddali, Bolman, Candel, et al., 2012; McClure et al., 2013; Smit et al., 2012) and decreasing alcohol intake (Riper et al., 2008; Riper et al., 2011; Rooke et al., 2010). Moreover, interventions to change multiple health behaviors have also been shown to be effective (Curioni \& Lourenco, 2005; Smeets et al., 2007; van Keulen et al., 2011; Vandelanotte et al., 2008). Studies also showed that computer-tailored interventions are more cost-effective than typical health care (D. N. Schulz, Smit, et al., 2014; Smit et al., 2013; van Keulen et al., 2010).

People of a low socioeconomic status (SES) often have unhealthy lifestyle behaviors (Lampert et al., 2013; Mackenbach et al., 2008). They often eat fewer fruits and vegetables (Hulshof, Brussaard, Kruizinga, Telman, \& Löwik, 2003), are less physically active (e.g., Gidlow et al., 2006), consume more alcohol (e.g., Naimi et al., 2003), and smoke more tobacco (e.g., Hiscock et al., 2012) compared to people with higher SES.

Although there is evidence that Web-based interventions are effective in improving health behavior, these interventions come with high dropout rates and the problem that participants often do not use the intervention as recommended (e.g., Cuijpers, van Straten, \& Andersson, 2008; Eysenbach, 2005; Moroshko, Brennan, \& O'Brien, 2011). 
Although studies have investigated characteristics of dropout and nonusage of eHealth interventions (e.g., Eysenbach, 2005; D. N. Schulz et al., 2012) it is equally important to know more about the participants who use these interventions as recommended. It is conceivable that people with different sociodemographic profiles may use these Web-based interventions differently because of general differences in online behavior and Internet usage between certain groups. This digital divide, for example, refers to unequal access to and use of the Internet among people with a lower SES (Aerschot \& Rodousakis, 2008; Brandtweiner, 2010; van Deursen \& van Dijk, 2011; Wei, Teo, Chan, \& Tan, 2011).

More than $90 \%$ of the general Dutch population has access to the Internet and $86 \%$ of Dutch people use the Internet every day (Akkermans et al., 2012; CBS, 2014a). Although the gap between people with and without Internet access seems to be closing, there is still a difference in Internet use between certain sociodemographic groups (Hargittai, 2010). People with a higher SES use the Internet more often than people with a lower SES to achieve personal development (e.g., getting a new job), whereas people with a lower SES use the Internet primarily for other purposes, such as entertainment (van Deursen \& van Dijk, 2014; van Dijk, 2005).

People with a higher educational level have been found to use the Internet more frequently to gain health-related information, for work, and for shopping or product information. People with a lower educational level use the Internet more often with other objectives, such as browsing the Web or playing online games (Costa-Font, Mossialos, \& Rudisill, 2009; Hargittai \& Hinnant, 2008; Howard et al., 2001; Wangberg et al., 2008). Educational level might also play a role in online behavior because most information on the Internet is written at a high literacy level whereas nearly half of the people do not understand this level of written information (Zarcadoolas, Blanco, Boyer, \& Pleasant, 2002). Moreover, employed people spend less of their leisure time online (van Deursen \& van Dijk, 2014). People with a lower income use the Internet more often for entertainment purposes, such as downloading music (Brodie et al., 2000; Martin \& Robinson, 2007; Močnik \& Širec, 2010), whereas people with a higher income spend more of their time online searching for news or information (van Deursen \& van Dijk, 2014).

Age- and gender-based differences in Internet use also exist. Although the majority (80\%) of Dutch people aged 65 years or older have access to the Internet (CBS, 2014c), they are less familiar with routine daily use of the Internet (Hargittai, 2010; Olphert \& Damodaran, 2013) and use the Internet primarily as an information source (S. Jones \& Fox, 2009) in contrast to younger Internet users who primarily understand the Internet as an entertainment medium (Dutton \& Blank, 2011). Males have been found to use the Internet more often, are more experienced with Internet use, and feel more comfortable with it (Cooper, 2006; Hargittai, 2010). This is in contrast to women, who spend less time online when having to take care of their family (Hargittai \& Shafer, 2006), but seek health information online more often than men (Brodie et al., 2000). In 
addition to these, other personal factors, such as a lower quality of life (Leung \& Lee, 2005; Mitchell, Lebow, Uribe, Grathouse, \& Shoger, 2011) or being married, are related to less Internet usage (Nie, Hillygus, \& Erbring, 2002).

Because SES is an important predictor of how people use the Internet (Hargittai, 2010), it is conceivable that people with a lower SES may not implement eHealth interventions as intended by the intervention developer and may be unlikely to follow intervention recommendations, which makes behavior change less likely (Elder, Ayala, \& Harris, 1999). However, because people with a lower SES are a high-risk group for unhealthy behaviors (Cowell, 2006; Hiscock et al., 2012; Margolis, 2013; Vlismas, Stavrinos, \& Panagiotakos, 2009), they are a highly relevant target group that might benefit from eHealth behavior change interventions. The same reasoning might hold for other personal characteristics, such as age, perceived health, or quality of life.

Therefore, this study investigates whether people are following the recommendations of how to use eHealth interventions. The purpose of our study is to identify personal and socioeconomic characteristics associated with recommended eHealth intervention use. Based on findings from the literature, we hypothesize that people with a higher education, who do not have paid work, those who have a lower income, who are younger, female, have a high perceived quality of life, and are not in a relationship are more likely to use the intervention as recommended.

\section{METHODS}

A detailed description of the study protocol has been published elsewhere (D. N. Schulz et al., 2011); only those study methodology details relevant to the study at hand are described here.

\section{Participants, Procedure, Study Design, and Intervention Content}

This study is part of a randomized controlled trial that was conducted in the Netherlands between 2009 and 2012. The study received ethics approval from the Medical Ethics Committee of Maastricht University and the University Hospital Maastricht (MEC) and has been registered by the Dutch Trial Register (NTR 2168). Participants were recruited through different Dutch Regional Health Authorities (RHAs) in the Netherlands (D. N. Schulz et al., 2011). These RHAs periodically monitor the health status, health behaviors, and related aspects of the adult population. At the end of this monitor, people were asked if they were interested in participating in this study. They were told that they would be invited to take part in a free online program that provides participants with tailored feedback about their health behavior. Internet access, a computer, basic Internet skills, and sufficient Dutch language skills were required preconditions for participating. The intervention consisted of two parts and 
focused on five health behaviors: fruit consumption, vegetable consumption, physical activity, smoking behavior, and alcohol intake. During the first part of the intervention, participants had to answer questionnaires about their health behaviors. The answers were used to provide participants with their personalized risk appraisal, which provided feedback by comparing the respondents' behavior to the Dutch guidelines defined for the five behaviors, such as (1) being physically active for at least 30 minutes on at least five days a week, (2) eating at least 200 grams of vegetables, (3) eating at least two pieces of fruit each day, (4) drinking no more than two glasses of alcohol a day (for men; one glass for women), and (5) not smoking at all.

The second part of the intervention consisted of five lifestyle modules. Participants who were interested in participation in the program received an email with their personalized link to log on to the computer-tailoring program. Based on the first part, the questionnaire assessment as part of the RHA monitor, participants were provided with tailored feedback concerning their behavior. They received an overview about all five behaviors and whether they met the guidelines or not. In the second part of the program, participants were asked to complete all modules for which they did not meet the guidelines. For example, in case a participant reported smoking and eating less than two pieces of fruit a day, he/she was advised to participate in the modules for smoking and fruit consumption. All modules included tailored feedback based on the determinants specified in the I-Change Model (de Vries et al., 2005): attitudes, social influence, self-efficacy, and preparatory and coping planning. The order of the modules was counterbalanced, either starting with preventive behaviors and addiction behaviors (i.e., physical activity/vegetable consumption/fruit consumption and alcohol intake/smoking), or vice versa (alcohol intake/smoking followed by physical activity/vegetable consumption/fruit consumption).

\section{Measures}

\section{Demographic Information}

The following demographic information were assessed: age, gender (1=male; $2=$ female), education (1=low: no education, primary, or lower vocational school; 2=middle: secondary vocational school or high school; $3=$ high: higher professional education or university), monthly income (1=<€1751; $2=€ 1751-€ 3050 ; 3=>€ 3050)$, work situation (1=no paid job; 2=paid job) (Verweij, 2008), family status ( $1=$ single; $2=$ relationship), number of persons living in the household, and country of birth (1=the Netherlands; $2=$ other).

\section{Health Status}

Participants were asked whether they suffered from (any of) the following diseases: diabetes mellitus, myocardial infarction, stroke, high blood pressure, other cardiovascular diseases, and/or cancer. Participants were categorized as ill (1=suffering 
from at least one of the diseases) or healthy (0). To assess quality of life, the Short Form Health Survey (SF-12) questionnaire was used (Gandek et al., 1998; Ware, Kosinski, \& Keller, 1996, 1998) (ranging from 18 to 48; based on a mean split of 24, we defined $0=a$ low quality of life score and $1=a$ high quality of life score).

\section{Health Behaviors}

All five health behaviors were assessed with the use of validated questionnaires. Physical activity was assessed with the Short Questionnaire to Assess Health-Enhancing Physical Activity (SQUASH) (Wendel-Vos, Schuit, Saris, \& Kromhout, 2003). Weekly vegetable intake (raw, boiled, baked, or salad), weekly fruit intake, and fruit juice consumption were assessed with the Food Frequency Questionnaire (FFQ) (RIVM, 2005). Alcohol consumption was assessed with the 5-item Dutch Quantity-FrequencyVariability (QFV) questionnaire (Lemmens, Tan, \& Knibbe, 1992). Smoking behavior was assessed by asking if, what (e.g., cigarettes, shag), and in what quantities participants smoke and their answers were converted into a score for tobacco consumption according to the recommendations by Mudde and colleagues (Mudde, Willemsen, Kremers, \& de Vries, 2006).

\section{Intervention Use}

We defined someone as using the intervention as recommended if he/she started with the suggested number of lifestyle modules based on his/her assessed behavior. Answering the first question within the specific module was defined as starting the module. For example, if a participant did not meet the guideline for smoking, vegetable consumption, and physical activity, this person was expected to start with three lifestyle modules (more than three modules were also counted as using the intervention as recommended) to be classified as a participant who uses the intervention as recommended. If this participant only started two or less modules, he/she was classified as not meeting the intervention recommendation.

\section{Statistical Analyses}

The data was analyzed with SPSS 20.0 (IBM Corp, Armonk, NY, USA). Descriptive statistics were used to describe participants' characteristics.

Frequency analyses were performed to identify the number of participants who used the intervention as recommended (1) and those who did not (0), as defined by the intervention recommendation. Only participants who got the advice to complete at least one module were included in analysis of intervention use as recommended. Differentiations were made between different subgroups for age, gender, education, income, working situation, health status, family status, and quality of life. We used chisquare tests to explore the differences among these groups.

Logistic regression analyses using the Enter method were used to predict intervention use as recommended among different personal characteristics. The 


\section{Chapter 4}

number of the received intervention modules, based on the amount of health behaviors that did not met the national guidelines, was used as a dependent variable. Separate logistic regression analyses were carried out dependent on the amount of lifestyle modules participants were advised to complete. One logistic regression analysis including all respondents was conducted with as-recommended program use as a dependent variable to detect characteristics that predict intervention use in general. Age, gender, level of education, income, employment status, family status, household size, country of birth, diseases, and quality of life were included as predictors in all models. The recommended number of modules was also taken into account in the model. Tests were performed at alpha $=.05$ to indicate statistical significance.

\section{RESULTS}

\section{Sample Characteristics}

A total of 1638 participants were included in this study (Table 4.1). Variables with missing data were not filled up (maximum missing values of $2.4 \%$ ). The mean age was 43.9 years (SD 12.6$)$ and slightly more men $(53.60 \%, 878 / 1638)$ than women $(46.40 \%$, $760 / 1638)$ participated in the trial. A minority of the participants had a low educational level $(10.84 \%, 174 / 1605)$. The majority had a middle income $(46.83 \%, 767 / 1606)$, were in a relationship $(75.94 \%, 1215 / 1600)$, and came originally from the Netherlands (95.27\%, 1531/1607). Most participants were healthy $(79.84 \%, 1283 / 1607)$ and reported a high quality of life (58.51\%, 935/1598). 
Table 4.1. Sample characteristics ( $N=1638)$.

\begin{tabular}{|c|c|c|c|}
\hline Characteristics & $\mathrm{n}(\%)$ & Mean (SD) & Range \\
\hline Age (years) & & $43.94(12.57)$ & $19-65$ \\
\hline \multicolumn{4}{|l|}{ Gender } \\
\hline Male & $878(53.60)$ & & \\
\hline Female & $760(46.40)$ & & \\
\hline \multicolumn{4}{|l|}{ Educational level } \\
\hline High & $700(43.16)$ & & \\
\hline Middle & $731(45.55)$ & & \\
\hline Low & $177(10.84)$ & & \\
\hline \multicolumn{4}{|l|}{ Income $(€)$} \\
\hline$<1750$ & $373(22.77)$ & & \\
\hline $1751-3050$ & $767(46.83)$ & & \\
\hline$>3051$ & $466(28.45)$ & & \\
\hline \multicolumn{4}{|l|}{ Working situation } \\
\hline Paid job & $1240(77.26)$ & & \\
\hline Nonpaid job & $365(22.74)$ & & \\
\hline \multicolumn{4}{|l|}{ Family status } \\
\hline Single & $385(24.06)$ & & \\
\hline In relationship & 1215 (75.94) & & \\
\hline Number of people in household & & $2.89(1.37)$ & $1-11$ \\
\hline \multicolumn{4}{|l|}{ Country of birth } \\
\hline The Netherlands & $1531(95.27)$ & & \\
\hline Other & $76(4.73)$ & & \\
\hline \multicolumn{4}{|l|}{ Disease status } \\
\hline III & $324(20.16)$ & & \\
\hline Healthy & $1283(79.84)$ & & \\
\hline Quality of life (SF12) & & $40.19(5.08)$ & $18-48$ \\
\hline High & $935(58.51)$ & & \\
\hline Low & $663(41.49)$ & & \\
\hline \multicolumn{4}{|c|}{ Number of modules recommended } \\
\hline 0 & $174(10.62)$ & & \\
\hline 1 & $451(27.53$ & & \\
\hline 2 & $585(35.71)$ & & \\
\hline 3 & $315(19.23)$ & & \\
\hline 4 & $100(6.11)$ & & \\
\hline 5 & $13(0.79)$ & & \\
\hline
\end{tabular}




\section{Intervention Use}

Figure 4.1 represents the percentages of participants who used the intervention in the recommended way. For example, 414 of 585 participants (70.8\%, red bar) who were advised to complete at least two lifestyle modules did not do so, 162 participants $(27.7 \%$, blue bar) followed the recommendation, and a minority $(9 / 585,1.5 \%$, green bar), attended more than two modules. These percentages indicate that the healthier their lifestyle and the fewer modules participants were advised to complete, the more participants followed the intervention guideline.

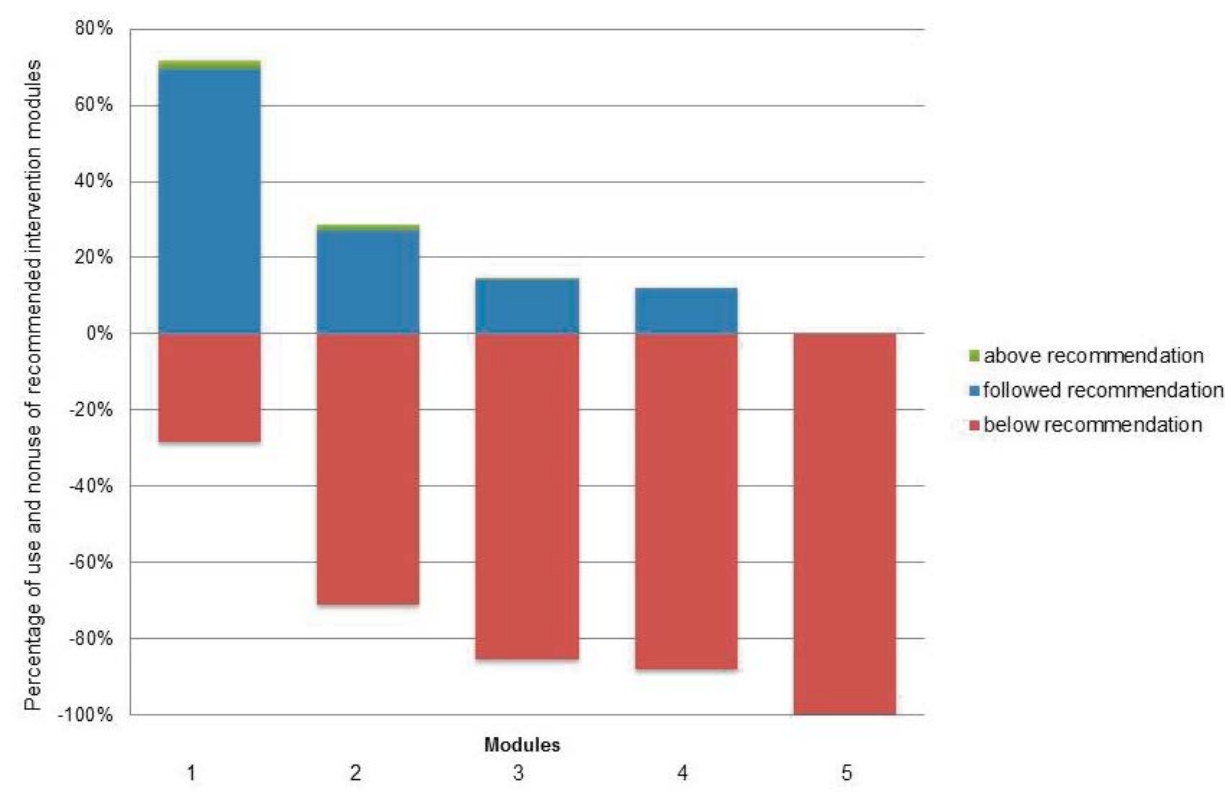

Figure 4.1. Percentage of participants who used the intervention in the recommended way.

\section{Intervention Use by Different Subgroups}

Table 4.4 gives an overview of the number of unhealthy behaviors and the number of started modules, differentiated by several personal variables. Figure 4.2 graphically summarizes the difference between the subgroups and the number of participants who did not comply with the intervention recommendation compared to those who used the intervention as recommended. 


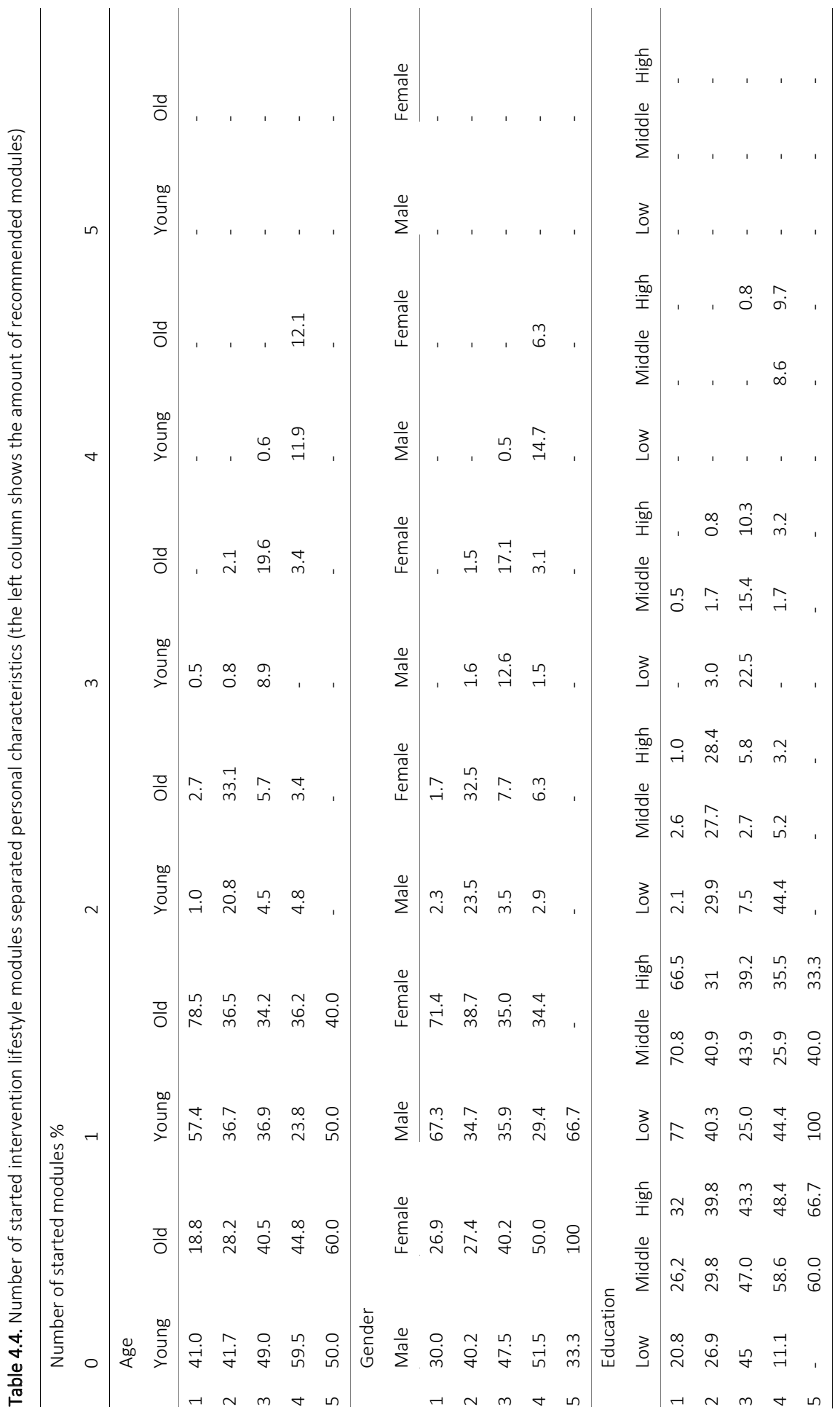




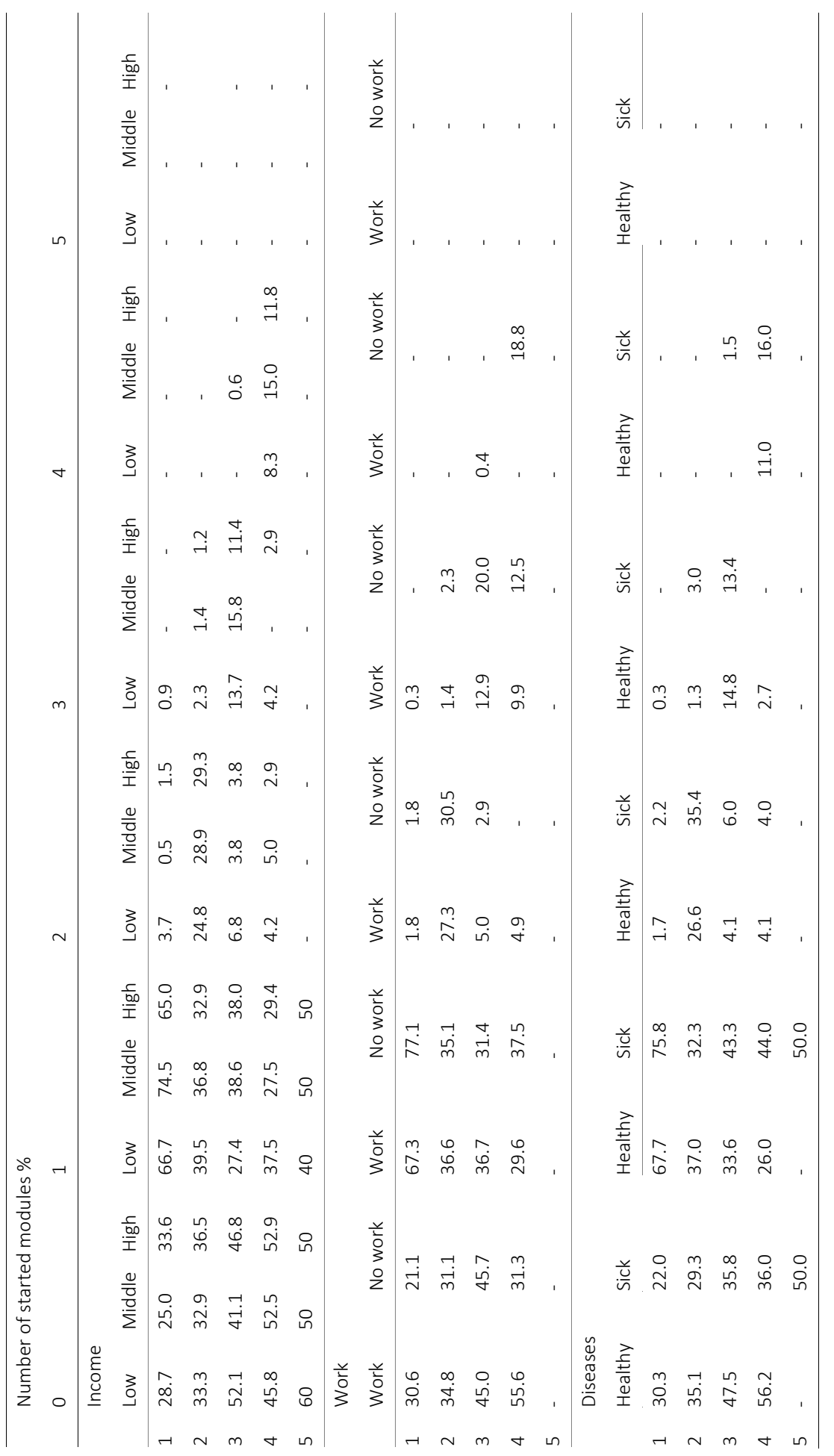


Who follows eHealth interventions as recommended?

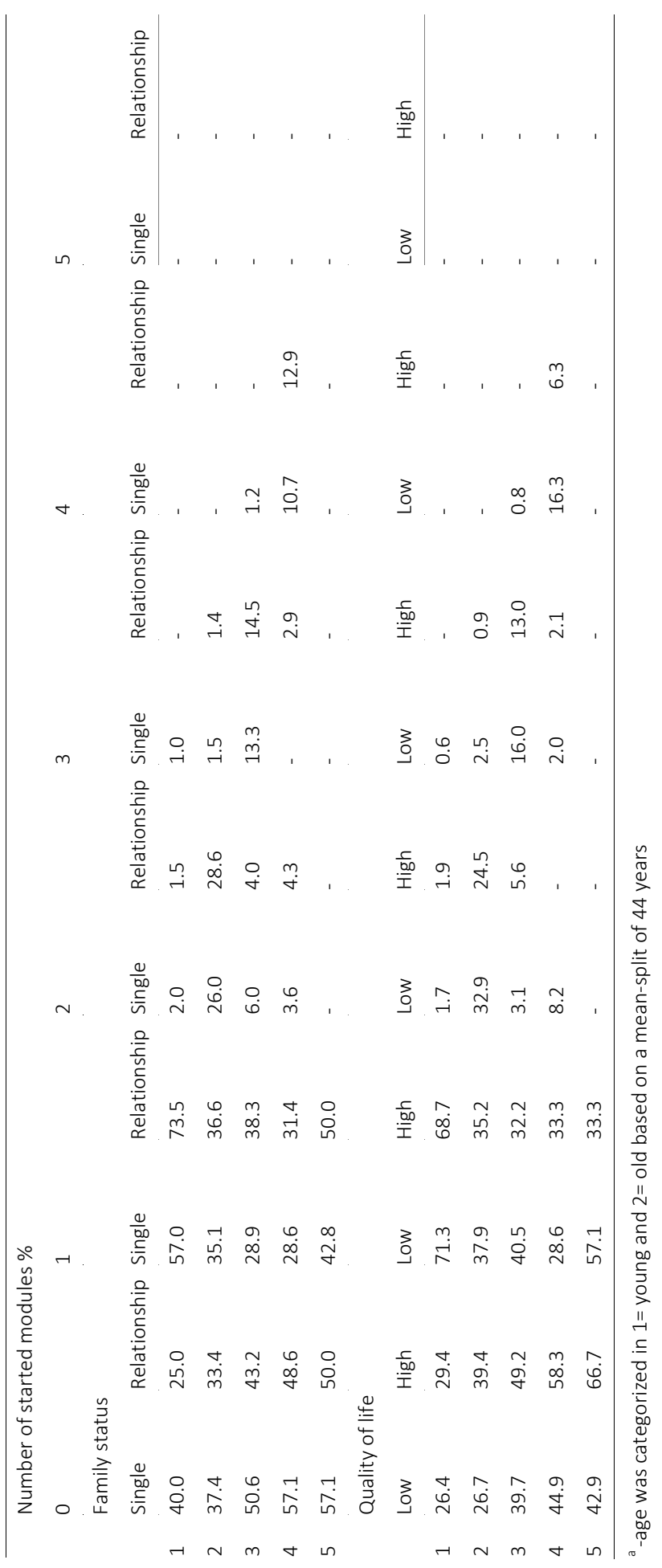




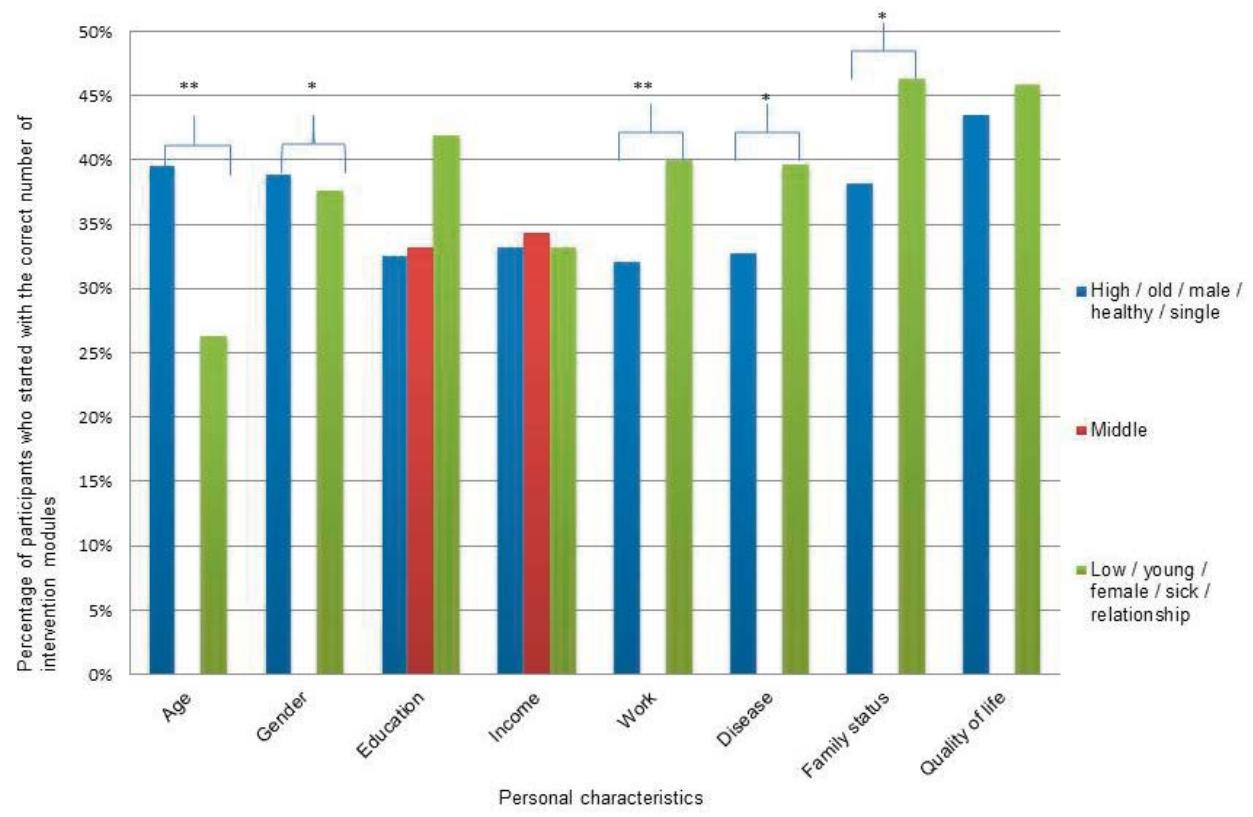

Figure 4.2. Percentage of participants who follow the recommendation to start with the correct number of intervention modules differentiated by education, income, work, age, gender, and disease status. Age was categorized as $1=y o u n g$ and $2=$ old based on a mean split of 44 years. ${ }^{*} p<.05,{ }^{* *} p<.001$.

Significantly more older (39.50\%, 361/914) than younger participants (26.3\%, 191/726; $\left.\chi_{1}^{2}=44.8, p<.001\right)$ and significantly more women $(50.66 \%, 385 / 760)$ than men $(38.84 \%$, $\left.341 / 878 ; \chi_{1}{ }_{1}=23.0, p<.001\right)$ used the intervention as recommended.

People with low education $(42.0 \%, 73 / 174)$ adhered best to the recommendation to participate in the suggested modules depending on their health behavior compared with participants with middle $(33.2 \%, 243 / 174)$ or high education (32.6\%, 228/700). However, no significant differences among these three educational levels were found with regard to recommended intervention use $\left(\chi_{1}^{2}=2.9, p=.23\right)$.

Participants with a low income $(33.24 \%, 124 / 373)$, middle income $(34.29 \%$, $263 / 767)$, and high income $(33.26 \%, 155 / 466)$ also did not differ significantly from one another with regard to intervention use $\left(\chi_{2}^{2}=0.6, p=.72\right)$.

However, participants without a paid job (40.0\%, 146/365) followed the recommendation of the intervention significantly more often than participants with a paid job (32.02\%, 397/1240; $\left.\chi_{1}^{2}=7.9, p=.01\right)$.

Those participants who were ill $(37.7 \%, 123 / 324)$ did use the intervention as recommended significantly more often compared to healthy participants $(32.74 \%$, 420/1283; $\chi_{1}^{2}=4.5, p=.02$ ). 
Participants in a relationship (46.34\%, 563/1215) followed the intervention recommendation significantly more often than single participants (38.2\%, 147/385 $\left.\chi_{1}^{2}=7.8, p=.003\right)$.

Finally, no significant differences were found for participants with a high quality of life $(43.5 \%, 407 / 935)$ compared to those with a low quality of life $(45.9 \%, 304 / 663 ; \chi$ $\left.{ }_{1}=0.9, p=.19\right)$.

\section{Predictors of Intervention Use}

As indicated in Table 4.2, higher age at baseline was a significant predictor of following the relevant recommendation to start with one or more modules. This was found for those who were recommended to follow one, two, and three modules, and in general. Age did not predict intervention use as recommended for respondents who were recommended to follow four lifestyle modules.

Being single significantly predicted recommended intervention use, but only for those participants who were advised to start with one module. However, none of the socioeconomic variables (education, income, and work) had a significant influence on the intervention use behavior regardless of the number of unhealthy behaviors. 


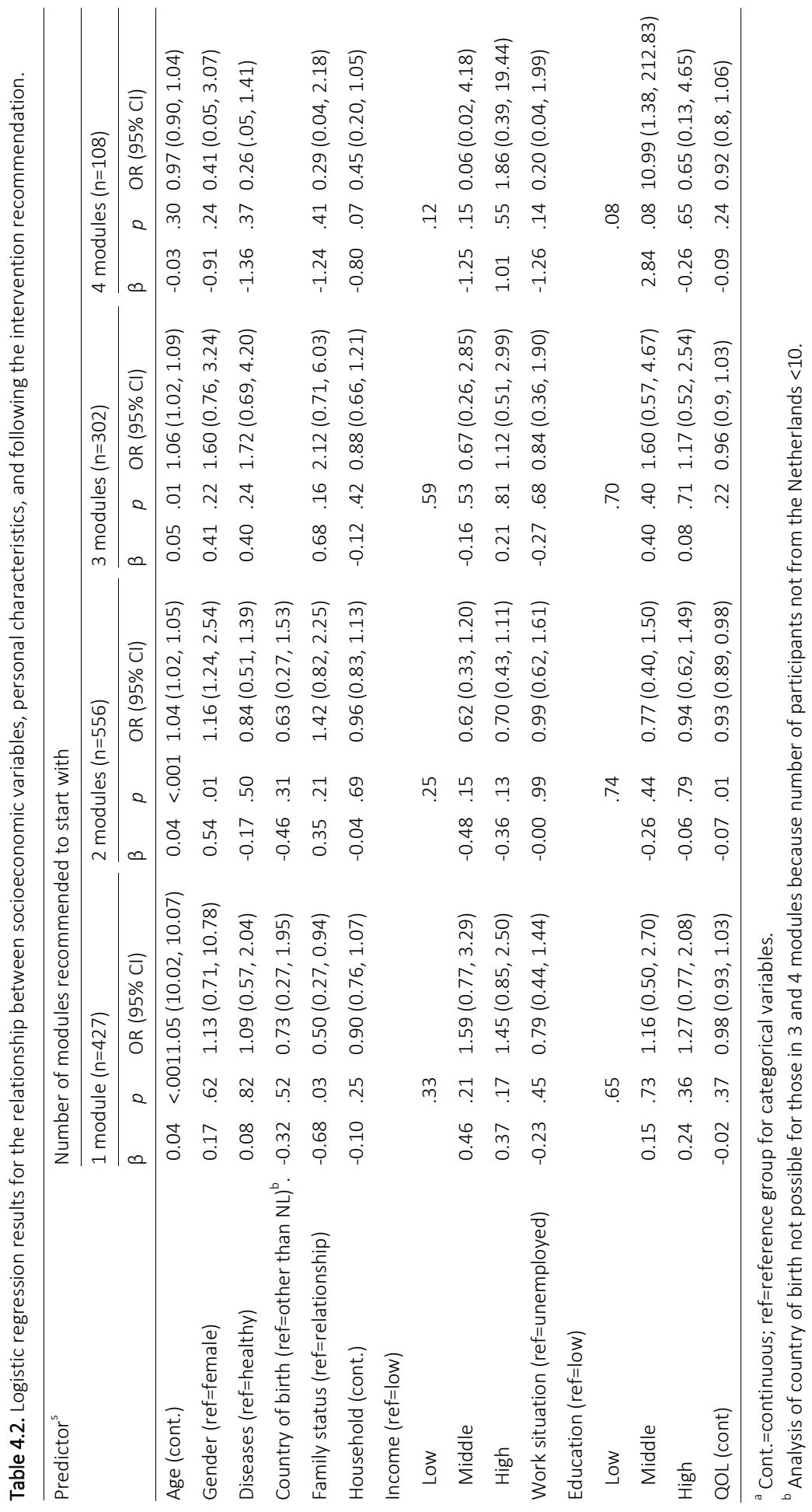


Being single significantly predicted recommended intervention use, but only for those participants who were advised to start with one module. However, none of the socioeconomic variables (education, income, and work) had a significant influence on the intervention use behavior regardless of the number of unhealthy behaviors.

A low quality of life (SF-12) was associated with being more likely to use the intervention as recommended for only people who received the advice to start with two modules. It should be noticed that the analysis of country of birth was not possible for the model with three and four modules recommendation because the number of participants not from the Netherlands smaller than ten.

The regression analysis of intervention use in general indicated that being older, female, having a lower quality of life, and given the recommendation to complete fewer lifestyle modules were significant predictors of intervention use according to recommendations (see Table 4.3).

Table 4.3. Logistic regression results for the relationship between socioeconomic variables, personal characteristics, and following the intervention recommendation within the complete sample ( $\mathrm{N}=1586)$.

\begin{tabular}{llll}
\hline Predictor & $\beta$ & $p$ & OR $(95 \% \mathrm{Cl})$ \\
\hline Age (cont.) & 0.04 & $<.001$ & $1.04(1.02,1.05)$ \\
Gender (ref=female) & 0.34 & .02 & $1.40(1.08,1.80)$ \\
Diseases (ref=healthy) & -0.18 & .71 & $0.94(0.67,1.31)$ \\
Country of birth (ref=not NL) & -0.26 & .40 & $0.77(0.42,1.41)$ \\
Family status (ref=in relationship) & 0.01 & .97 & $1.00(0.67,1.46)$ \\
Household (cont.) & -0.09 & .07 & $0.91(0.82,1.01)$ \\
Income (ref=high) & & & \\
Low & & .67 & \\
Middle & -0.16 & .47 & $0.86(0.56,1.31)$ \\
High & 0.01 & .98 & $1.06(0.74,1.34)$ \\
Work situation (ref=unemployed) & -0.15 & .35 & $0.86(0.62,1.19)$ \\
Education (ref=high) & & & \\
Low & & .78 & \\
Middle & & .79 & $1.06(0.68,1.67)$ \\
High & 0.06 & .58 & $1.08(0.82,1.43)$ \\
QOL (cont.) & 0.08 & .002 & $0.96(0.93,0.98)$ \\
Module recommendation (cont.) & -0.04 & $<.001$ & $0.20(0.17,0.24)$ \\
\hline
\end{tabular}

${ }^{\text {a }}$ Cont.=continuous; ref=reference group for categorical variables. 


\section{DISCUSSION}

\section{Main Findings}

Because eHealth intervention use as recommended increases the effectiveness of behavior change (Elder et al., 1999), it is of high importance that people at a high risk of unhealthy lifestyle behaviors use those interventions in such a way. Our analysis of a sample of the general Dutch population revealed that there was a difference in intervention use among people grouped by different personal characteristics.

Contrary to earlier findings regarding Internet use and age (e.g., Costa-Font et al., 2009; Hargittai, 2010), we found that more older than younger participants used the intervention as recommended. It might be possible that older people were less familiar with eHealth interventions and, therefore, gained more information that was new and relevant to them resulting in more frequent use of the intervention modules.

Women in our study used the intervention as recommended more often, which could be explained by the fact that women use the Internet to seek health information more frequently than men (Cotten \& Gupta, 2004). Females tend to be more interested in health topics (Cline \& Haynes, 2001; S. Fox \& Fallows, 2003) and rely more often on the Internet as a trustful source (Bell, 2014; Costa-Font et al., 2009). These explanations might be possible reasons why women used the intervention as recommended more frequently compared to men in our study. In addition, males have been found to evaluate the Internet as a less valuable source of health information than women do (Costa-Font et al., 2009), which might include eHealth interventions, and this may be another reason for the lower intervention adherence by men in our study.

Participants within a relationship have been found to use the intervention as recommended more frequently compared to singles. People within a relationship have been found have healthier behavior and health might be something in their interest which could explain why they are more interested in using the intervention (Umberson \& Montez, 2010). Further research should explore the importance of family status as well as health behaviors of other family members in more depth because it might be that family members are more likely to behave alike, which might ultimately affect (the need for) intervention use.

People with a lower educational level used more intervention modules those with a higher education level did. This result is surprising because it is known from the literature that higher-educated people spend more time online to seek health information health information (Jensen, King, Davis, \& Guntzviller, 2010; Wangberg et al., 2008). One explanation is that the lower-educated participants may lack prior knowledge and may have used this intervention to gain more knowledge about a healthy lifestyle (Costa-Font et al., 2009). Another explanation could be that people with a lower SES use the Internet primarily to gain information, whereas people with a high SES make use of different sources, including professionals or their social 
environment, and thus rely less on the Internet for information (Bell, 2014). But these results must be interpreted with caution because although we found higher asrecommended intervention use, education was not a significant predictor within the regression analysis. Furthermore, our data indicated that income is not a predictor for recommended intervention use, which might indicate that income level might not be important with regard to intervention use as recommended.

This study revealed that unemployed people used the intervention as recommended more frequently. Participation in the intervention is time-consuming and it may be that employed people adhered less to the intervention recommendations because they had less leisure time. Previously, van Deursen and van Dijk (van Deursen \& van Dijk, 2014) reported that unemployed people spent more time online than employed people did.

The fact that participants who report to have a disease used our intervention more frequently is in-line with previous literature findings. Individuals who perceive themselves as more ill have been found to use the Internet as a source of health information (Brashers, Goldsmith, \& Hsieh, 2002; Bundorf, Baker, Singer, \& Wagner, 2004). This might also be an explanation for our finding that participants with a lower level of quality of life used the intervention as recommended. It might be plausible that these participants look for health information and tips about how to change their lifestyle to gain a better health condition and a higher quality of life.

We also found the more modules recommended to complete, the fewer were done by participants. Following the recommendations of an eHealth intervention requires a significant investment of time for reading and processing information and interacting with the program. Participants who received the recommendation to use many modules might be at greater risk of being overwhelmed by those requirements. If an eHealth program demands too much cognitive effort from their participants, ego depletion (Baumeister, Heatherton, \& Tice, 1994; Baumeister, Muraven, \& Tice, 2000) can arise and participants might be more inclined not to use the program as recommended.

To summarize, we have found differences in intervention use as recommended among participants with different personal characteristics. We know now that especially younger people, males, people who have a job, people with illnesses, and singles did not use the intervention as recommended. Furthermore, our analysis revealed that being older, female, having a low quality of life, and a healthier lifestyle are predictors of intervention use as recommended when all personal characteristics are taken into account.

\section{Strengths, Limitations, and Further Implications}

One of the strengths of this study is the multiple-behavior approach because previous studies have demonstrated that those interventions have a high impact on behavior change (D. N. Schulz, Kremers, et al., 2014). Furthermore, we assessed several 
indications to measure SES, which allows us to compare the impacts of education, income, and occupational status.

In addition to the randomization of the started behaviors, either preventive or addictive modules, one of the limitations of this study is that participants could not choose on their own with which module they wanted to begin with in the given module block. This might have increased the risk of participants not using the intervention because they might have disliked a given sequence. Furthermore, a predefined order of the modules may have led to reduced feelings of choice. We were not able to analyze potential consequences of this reduction and further studies may explore this issue at greater depth. Participants could have also misinterpreted a predefined order as an order of importance. Providing the module about fruit consumption first and then smoking might have created the impression that changing fruit consumption is more important than smoking cessation. Another limitation of the predefined order of the modules might be that some participants had to start with a behavior that they experienced as difficult, which may have led to reduced motivation to complete the full program. Future intervention studies should study under which conditions the utilization of a simultaneous strategy favoring multiple behaviors simultaneously or sequentially are preferred by participants.

Although this study used the term "following interventions as recommended," we can only say for sure that participants started with the recommended amount of modules and not whether they finished the modules or how they used the modules. Next, the aim of the study was to look at differences in people of this intervention group in regards to following intervention recommendations. Yet, participants of an intervention are often preselected, implying that people who are healthier and more interested in health are more willing to participate.

Another limitation of the study at hand is the use of self-reported questionnaires, which could result in an overestimation of healthy lifestyles and participants being given the recommendation to start with fewer intervention modules. Self-reported questionnaires with regard to education, income, and working situation might also result in an underestimation of people with a low SES (e.g., false information about income).

Finally, we did not analyze whether following the intervention recommendation resulted in behavior change or not. However, in one of our studies (D. N. Schulz, Kremers, et al., 2014), it was investigated that a longer visiting time and greater number of visits in the intervention resulted in higher likelihood of behavior change. To our knowledge, this is one of the first studies focusing on socioeconomic and personal characteristics as a predictor of recommended intervention use. Because we found that older participants, females, unemployed people, ill participants, and people in a relationship more often use the intervention as recommended, we should ask ourselves what strategies can be taken to make interventions more attractive to use for those who did not use the intervention as recommended. Our intervention included several 
strategies that are known to increase intervention use, such as giving tailored feedback, using goal-setting strategies, action planning, self-efficacy monitoring, and the use of reminders (Brouwer et al., 2011; Crutzen, de Nooijer, et al., 2011). On the other hand, the intervention might be more attractive if interactive elements and communication tools to facilitate social support were used, when involving the social environment, or if entertaining elements (e.g., additional games, quizzes) were added (Brouwer et al., 2011; Crutzen, de Nooijer, et al., 2011). Furthermore, the health modules were very similar in terms of structure and type of feedback, and it might be that participants disliked the repetition (which also increases participants' burden). Future research is warranted to investigate whether improving the flow experiences of participants by using strategies to attract participants' attention and make interventions more entertaining increases recommended intervention use (Csikszentmihalyi, 1997; Webster, Trevino, \& Ryan, 1994).

\section{CONCLUSION}

Our findings indicate that different subgroups use eHealth interventions in different ways. The more frequent as-recommended intervention use by unemployed, older, and ill participants may be an indication that these eHealth interventions are attractive to people with a greater need for health care information. Therefore, computer-tailored eHealth interventions might be a promising tool to increase health status and maintain healthy lifestyles.

\section{CONFLICTS OF INTEREST}

Hein de Vries is the scientific director of Vision2Health, a company aimed at implementing evidence based eHealth programs. All other authors have no conflicts of interests. 



\section{ABSTRACT}

Background: In order to improve the transition from an intention to a change in health behaviour, action planning is a frequently used behavioural change method. The quality of action plans in terms of instrumentality and specificity is important in terms of supporting a successful change in health behaviour. Until now, little has been known about the predictors of action plan generation and the predictors of high quality action plans and, therefore, the current study investigates these predictors.

Method: A randomised controlled trial was conducted to improve physical activity (PA) and fruit and vegetable (FV) consumption using a Web-based computer tailored intervention. During the eight-week intervention period, participants in the intervention arm $(n=346)$ were guided (step-by-step) to generate their own action plans to improve their health behaviours. Demographic characteristics, social cognitions, and health behaviour were assessed at baseline by means of self-reporting. Whether participants generated action plans was tracked by means of server registrations within two modules of the intervention.

Results: The action planning component of the intervention regarding physical activity and fruit and vegetable consumption was used by $40.9 \%$ and $20.7 \%$ of the participants, respectively. We found that participants who were physically active at baseline were less likely to generate action plans concerning physical activity. With regards to generating fruit and vegetable action plans, participants with a high risk perception and a strong intention to eat fruit and vegetables on a daily basis made more use of the action planning component for this behaviour. Finally, the large majority of the action plans for physical activity (96.6\%) and fruit and vegetable consumption (100\%) were instrumental and about half of the action plans were found to be highly specific ( $P A=69.6 \% / F V=59.7 \%)$. The specificity of the action plans is associated with having a relationship and low levels of negative outcome expectancies.

Conclusion: Risk perception and intention are predictors of using the application of action planning. Increasing the motivation to change behaviour should be prioritised in interventions concerning changes in health behaviour before participants are asked to generate action plans. This would also make the intervention suitable for unmotivated people. For those participants who already perform the desired health behaviour prior to the intervention, action plans might be less relevant. Nevertheless, using a guided step-by-step approach to generate action plans resulted in highly instrumental and specific action plans and might be integrated into other interventions concerning changes in health behaviour.

Trial Register: Netherlands Trial Register: NTR 3706, ClinicalTrials.gov: NCT01909349 Keywords: Physical activity; fruit and vegetable consumption; action plan; plan quality; instrumentality; specificity 


\section{INTRODUCTION}

Even highly motivated people can have problems in translating their intentions into a successful change in health behaviour (Orbell \& Sheeran, 1998). Action planning has been identified as an important method to overcome this "intention-behaviour-gap" (Bartholomew, Parcel, Kok, Gottlieb, \& Fernández, 2011; Sutton, 2008) by improving selfregulatory skills (Schwarzer, 2008). Action plans (AP) specify precisely when, where, and how an intended health behaviour will be carried out (Fennis, Adriaanse, Stroebe, \& Pol, 2011; Gollwitzer, 1993, 1999). When asking people to generate APs, such people should be encouraged to think about the context in which the desired behaviour will be performed. Due to these cues to action, such APs should work as a reminder to act (in terms of time and place) (Hagger \& Luszczynska, 2014); even when other self-regulatory skills and memory capacity are low, planning contributes to habit formation (Chatzisarantis, Wood, Stiff, \& Hagger, 2010; Parks-Stamm, Gollwitzer, \& Oettingen, 2007).

Generating APs has been demonstrated to increase the translation from intention to behaviour for different health behaviours such as PA (de Vet, Oenema, \& Brug, 2011; Gellert, Ziegelmann, Lippke, \& Schwarzer, 2012; Sniehotta, Nagy, Scholz, \& Schwarzer, 2006; Sniehotta, Scholz, \& Schwarzer, 2006) and a healthy diet (Adriaanse, Vinkers, De Ridder, Hox, \& De Wit, 2011; Thompson et al., 2015). The theoretical method of action planning is derived from several social-cognitive health theories such as the Health Action Process Approach (HAPA) (Schwarzer, 2004, 2008; Schwarzer, Luszczynska, Ziegelmann, Scholz, \& Lippke, 2008). It can be easily applied (Bartholomew et al., 2011) and its application is understandable to users (Hagger \& Luszczynska, 2014) and, hence, it is often applied to Web-based interventions to encourage a change in health behaviour (Hagger \& Luszczynska, 2014; Webb, Joseph, Yardley, \& Michie, 2010).

The HAPA assumes that health behaviour is determined by social cognitions like risk perception, self-efficacy, and outcome expectancies as more distal determinants that influence intention as a more proximal determinant. Intention influences behaviour via action planning. HAPA assumes that action planning is determined by self-efficacy and intention (Schwarzer, 2008). It has been shown that people who have a high selfefficacy to perform a specific behaviour (Gillholm \& Gärling, 1997; Gollwitzer, Odenthal, \& Wieber, 2010; Scholz, Sniehotta, Schüz, \& Oeberst, 2007), have positive outcome expectations, have strong intentions (de Vries, Eggers, \& Bolman, 2013; Lippke, Ziegelmann, \& Schwarzer, 2004), are older, and are female (de Vries et al., 2013) are more engaged in making APs. Furthermore, people with a low educational level have been found to use Web-based health interventions less frequently than is recommended in terms of participating in the recommended modules (Reinwand, Schulz, et al., 2015). Those in a relationship and those who are unemployed follow intervention recommendations more closely compared to singles and employed people (Reinwand, Schulz, et al., 2015). Therefore, it might be possible that people with 
different educational levels, different relationship statuses, and different working conditions make different use of the intervention AP application.

While APs have been found to be a useful tool to improve a change in health behaviour, especially for fruit and vegetable (FV) consumption and physical activity (PA) (Belanger-Gravel, Godin, \& Amireault, 2013; Lippke et al., 2004; van Osch et al., 2009), the quality of the APs might also have an influence on the change in health behaviour. The quality of APs can be described in terms of instrumentality and specificity (Gollwitzer, Sheeran, \& Mark, 2006). Instrumental plans are goal-directed and describe an action that will result in the desired behaviour. For example, the desired behaviour is to be physically active and the AP focuses on using a bicycle to go to work. An AP would not be instrumental if the plan is not linked to the desired behaviour, such as eating less snacks. Furthermore, APs can vary in the degree of details provided (i.e., when, where, how, and with whom) (Gollwitzer \& Oettingen, 2013) and studies have revealed that the more specific an AP, the more likely it is that the intended behaviour will be performed (de Vet et al., 2011; Elfeddali, Bolman, \& de Vries, 2013; van Osch, Lechner, Reubsaet, \& de Vries, 2010; Ziegelmann, Lippke, \& Schwarzer, 2006).

Unlike social cognitions, personal characteristics of people generating an AP and the AP quality, has not been discussed in existent literature. However, it is important to understand if a practical application to generate APs is used equally between different subgroups (i.e., higher and lower educated).

The aim of this study is to evaluate the use of the intervention and the quality of the generated APs. Therefore, our first research question is: How are the modules from a Web-based computer tailored intervention used and what are the predictors of intervention use? The second question is: Which predictors of generating APs for both behaviours can be identified? The third question is: What is the quality of the generated APs in terms of instrumentality and specificity? Finally, our last research question is: What are the predictors of AP specificity?

\section{METHODS}

\section{Ethical approval}

The data analysis for the present paper was collected from a randomised controlled trial that tested the effectiveness of a Web-based tailored intervention to facilitate PA and FV consumption. A study protocol about the study design and intervention has been published elsewhere (Reinwand, Kuhlmann, Wienert, de Vries, \& Lippke, 2013); in the following part, only the relevant details for the analyses in this paper will be described. The study received ethical approval in the Netherlands by the Medical Ethics Committee of Atrium Medical Centre Heerlen (METC number 12-N-124) and in Germany by The German Society for 
Psychology (DGPs; EK-A-SL 022013). All participants were asked to sign online informed consent, explaining the voluntariness and anonymity of their participation.

\section{Participant recruitment}

Participants for this study were recruited in the Netherlands and Germany by advertising in hospitals, in newspapers, on social media networks, and by using research panels (Flycatcher in the Netherlands and Dr. Grieger \& Cie in Germany). Participants aged 20 and older who were interested in reducing their cardiovascular risk by improving their PA and FV consumption were invited to participate.

\section{Study design and intervention content}

Participants were allocated randomly by the computer software TailorBuilder (TailorBuilder, 2015) (a programme for Web-based computer tailored interventions) into either the intervention group or the waiting list control group. We only made use of the information gained from the intervention group for the current study. At baseline, all participants filled in an online baseline questionnaire and, thereafter, participants in the intervention group gained access to the Web-based intervention.

The intervention consisted of an eight-week online programme in which participants were encouraged to log in once a week to participate in a specific module. The first four modules addressed PA and the last four modules focused on FV consumption. During the first module, participants were asked to generate a general goal with regards to PA (FV consumption was dealt with in the $5^{\text {th }}$ module). In the second module, participants were guided to generate APs as precisely as possible; for which they were encouraged to generate "which day", "at what time", "how long", "where", and "with whom" they were planning to be physically active (or eating FVs during module six). Participants were asked to evaluate their own APs using the "PEPP-rule" (Figure 5.1). PEPP means "Proper", "Efficient", "Practicable", and "Precise" and this rule is used to avoid unrealistic planning (Fleig, Pomp, Schwarzer, \& Lippke, 2013). In each module, participants received tailored feedback with regards to their health behaviours and planning progress. All participants were asked to formulate APs. Those who fulfilled the behavioural guidelines received the feedback that planning is a useful tool to maintain the desired behaviour in the long run. Participants who did not reach the behavioural guidelines received the feedback that planning would help to translate their intentions into behaviour even in difficult situations. Participants were able to reflect on their APs and adjust them if necessary. The data from the APs that participants generated during the two intervention modules has been used for this paper (Reinwand et al., 2013). 


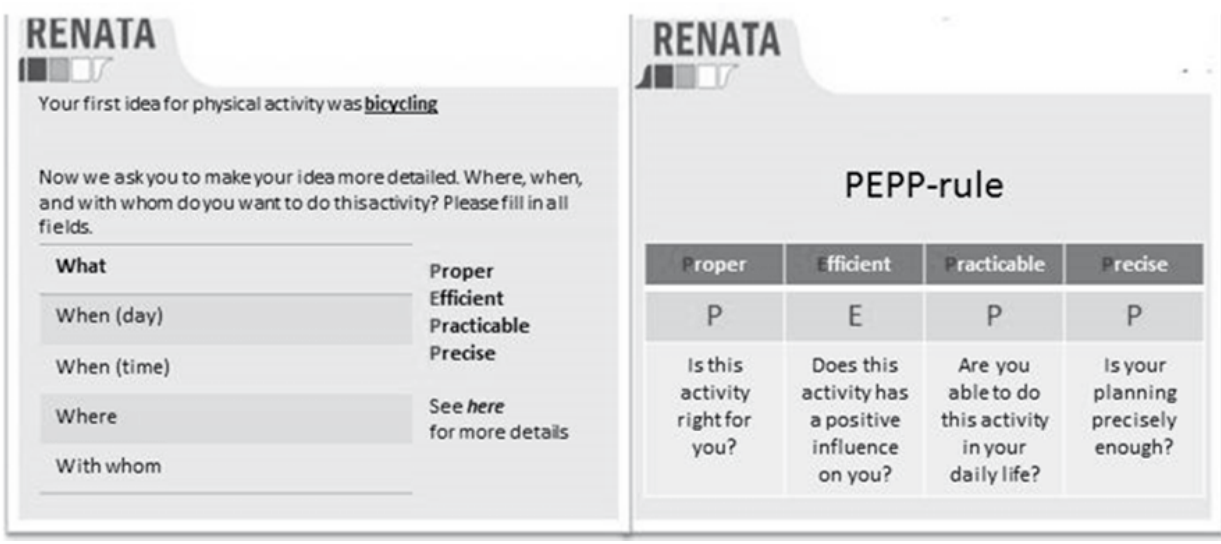

Figure 5.1: Example of how to generate APs within the intervention (PEPP-rule based on Fleig et al., 2013)

\section{Measures}

The following demographic information was assessed: age, gender (1=male, 2=female), educational level (1=low, $2=$ middle, $3=$ high), relationship ( $1=$ single, $2=$ partnership), and working situation (1=unemployed, $2=$ employed).

To assess health behaviour, the level of $P A$ was measured using the short version of the International PA Questionnaire (IPAQ) (Craig et al., 2003; Mäder, Martin, Schutz, \& Martil, 2006). Assessing FV consumption during the past seven days was done with the use of the Behavioral Risk Factor Surveillance System (BRFSS) questionnaire which asks participants to count the number of portions of fruit, vegetables, and salads they ate (on average) during a day (Rafferty, Anderson, McGee, \& Miller, 2002).

The recommendation to be physically active at least five days in a week for at least 30 minutes per day (WHO, 2010) was used to categorize participants in terms of whether they fulfil the recommendation (2) or not (1). The same coding was used for FV consumption. Participants who ate (on average) five portions of FVs each day were classified as being compliant with the recommendation (Boering et al., 2012).

Risk perception with regards to cardiovascular diseases was measured in terms of five items, including: "How likely is it that you will sometime in your life have: ..." "... a high cholesterol level?" or "... a stroke?" $(\Omega=0.88, \mathrm{Cl}=0.87-0.90)$. Possible responses ranged from $1=$ totally disagree to $7=$ totally agree.

Outcome expectancies were measured in terms of two positive items $(r=0.69)$ and two negative items $(r=0.34)$ concerning PA. Similarly, FV outcome expectancies were assessed in terms of two positive $(r=0.64)$ and two negative $(r=0.53)$ outcome expectancies. For example: "Being physically active for at least 30 minutes a day for at least five days a week will make me feel better", "Eating 5 portions of FV a day will be good for my health" (D. N. Schulz, Kremers, et al., 2014). Items could be answered on a 7-point Likert scale (1=totally disagree -7 =totally agree). 
Self-efficacy was assessed in terms of five items for PA $(\Omega=0.88, \mathrm{Cl}=0.86-0.89)$ and five items for $\mathrm{FVs}(\Omega=0.91, \mathrm{Cl}=0.90-0.92)$. Examples of such items include: "I am certain that I can be physically active a minimum of 5 days a week for 30 minutes even it is difficult", "I am certain that I can eat at least 5 portions of fruit and vegetables a day even if it is difficult" (motivational) (Nigg, 2005). "I am certain that I can be physically active permanently at a minimum of 5 days a week for 30 minutes ... / "I am certain that I can permanently eat 5 portions of fruit and vegetable a day..." "... even if it takes a lot of time till I am used to do it" (maintenance) (Luszczynska \& Sutton, 2006), and "I am certain that I can again be physically active a minimum of 5 days a week for 30 minutes / I am certain that I can again eat 5 portions of fruit and vegetables a day ..." "even if I changed my concrete plans several times" (recovery) (Luszczynska \& Sutton, 2006). Possible responses ranged from 1=totally disagree to 7=totally agree.

Intention to be physically active was assessed in terms of two single items: "On 5 days a week for 30 minutes (or a minimum of 2.5 hours per week), I have the intention to perform..." 1: "...intensive physical activity", 2 "... moderate physical activity". Intention about FV consumption was assessed in terms of one item: "I seriously intend to eat at least 5 portions of fruit and vegetable daily" with possible responses ranging from totally agree $(=7)$ to totally disagree $(=1)$ (Lippke, Ziegelmann, Schwarzer, \& Velicer, 2009).

To determine the AP quality in terms of specificity and instrumentality, information from the intervention itself was used. Whether participants generated a meaningful AP in module two (about PA) or in module six (about FV consumption) was coded as whether an AP was generated $(=2)$ or not $(=1)$. To assess the quality of the APs, we distinguished between instrumentality and specificity. We considered an AP to be instrumental when participants generated a goal-directed action (1). A plan was not instrumental (0) when the described action would not result in improving PA or FV consumption. Specificity was only defined in terms of instrumental APs. We categorised specificity into three categories: A plan is not specific (0) when participants had only generated what to do. Medium-specific (1) is defined as a plan that provided additional details about the time and day on which the action will be performed. A plan was considered highly specific (2) when it also described where and with whom the action would be done (participants were also allowed to comment that they wished to perform their behaviour alone) (van Osch et al., 2010). The APs were independently coded by two researchers. In 14 out of 438 cases, there was a discrepancy between the coding of the researchers, which was resolved by means of discussion.

\section{Statistical analysis}

The data was analysed using SPSS software version 21 (IBM Corp, Armonk, NY, USA). Descriptive statistics were used to describe study sample characteristics. With regards to our first research question, a stepwise linear regression analysis was used to assess 
the predictors of intervention use whereby intervention use was defined in terms of the number of modules that participants completed (possible range: 0-8 modules).

In line with the HAPA model, variables were included in three steps: the first model contained only socio-demographic variables (age, gender, relationship, working situation, and educational level), the second model included (in addition to the first model) risk perception, outcome expectancies, self-efficacy, and whether participants' behaviours are in line with the specific recommendation (yes=2 no=1). The final model also included intention (in addition to the first and second model).

Regarding research question two, a stepwise logistic regression analysis was used to assess predictors of action planning (yes=2 or no=1), by using the same three above mentioned models. One analysis was undertaken using action planning for PA as a dependent variable and one analysis was undertaken using action planning for FV consumption as a dependent variable.

In order to describe the quality of the generated APs, descriptive statistics were used. Next, a stepwise linear regression analysis was undertaken to assess the predictors of making specific APs (research question four) for PA and for FV consumption, using the same three above mentioned models. When participants generated two plans for one of the behaviours, a mean score was calculated for the specificity of the plans. We excluded APs that were not classified as instrumental in the analysis such as: "undertake renovations", "doing some arm-wrestling", or "no idea" because they are irrelevant to this study ( $n=9$ for PA, none for FV). A p-value of .05 was defined as the level of significance.

\section{RESULTS}

\section{Participants' characteristics}

Table 5.1 shows the characteristics of the 346 participants in the intervention group at baseline. The mean age is 50.91 years (range 22-84) and the sample includes more females $(65.2 \%)$ than males. $46.0 \%$ of the participants had a mid-educational level, $77.4 \%$ of the participants were in a relationship, and $63.1 \%$ were employed. Regarding the compliance with the behavioural guidelines, we have found that 153 (44.2\%) of the study participants were physically active for at least 30 minutes a day on at least five days a week, and that 144 (41.7\%) of the study participants ate five portions of fruits and vegetables a day. 
Generating and predicting high quality action plans

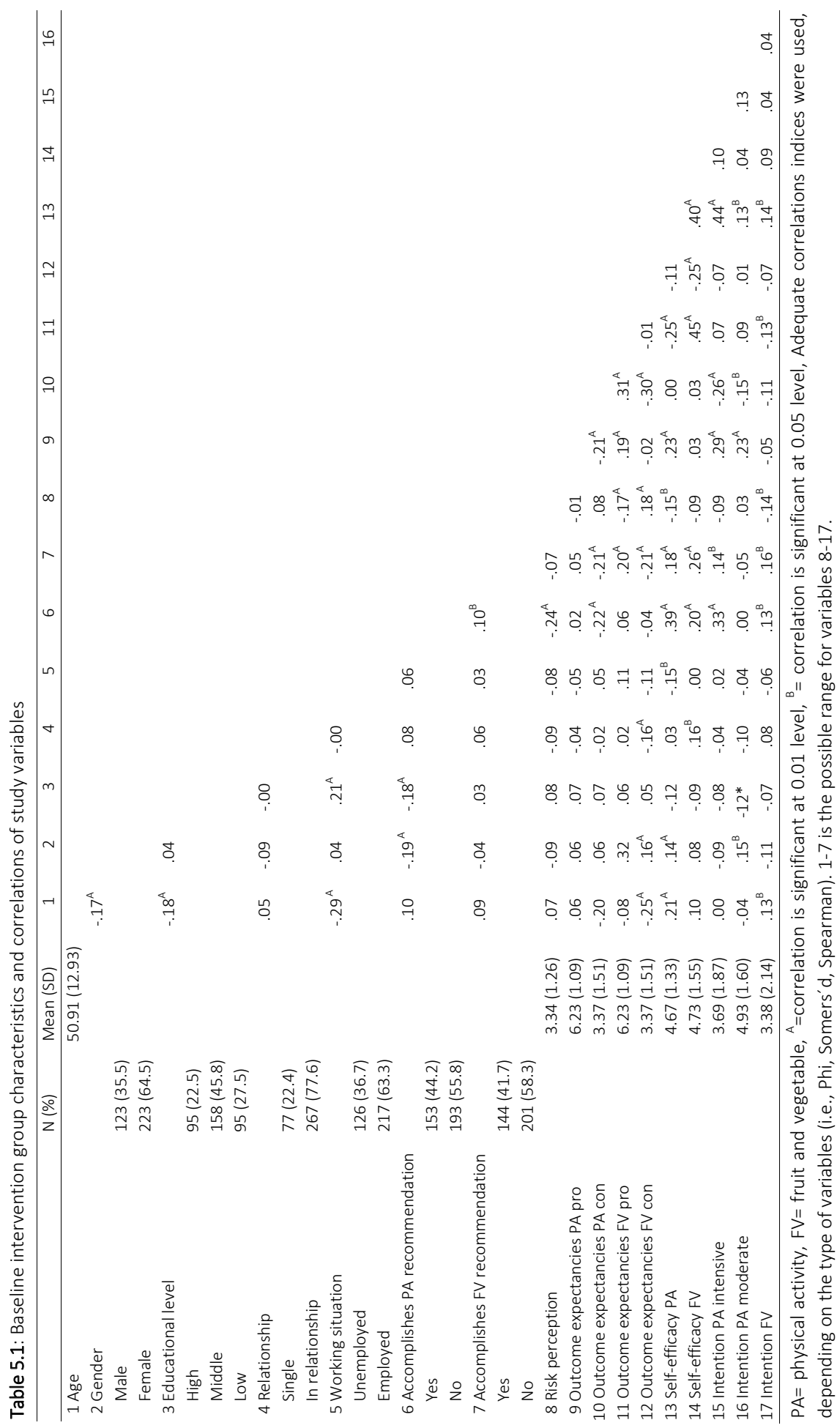


Figure 5.2 gives an overview about the percentage of participants from the intervention group that took part in the individual intervention module. The participation rate continuously decreased each week, beginning with $90.8 \%$ in the first module and ending with $19.9 \%$ in the last module.

Furthermore, data derived from the online intervention shows that 153 (44.2\%) of the participants made an AP regarding PA (module 2) and 76 (22.0\%) participants made an AP regarding FV consumption (module 6). It is possible that participants only generated actions plans, but did not complete the whole module. Hence, there is a difference between the number of generated APs and the percentage of participants who completed a module.

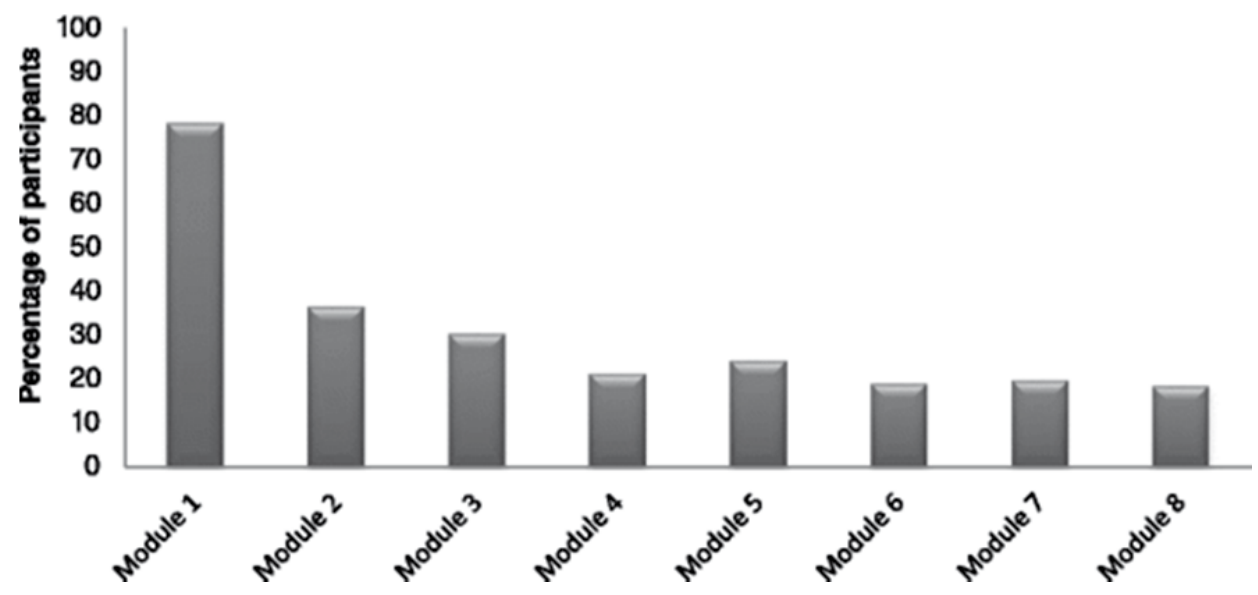

Figure 5.2: Intervention use rate of completed modules

Predicting the intervention use, we ran a stepwise linear regression analysis. The third model shows that intervention use was significantly predicted by participants being in a relationship, having a higher risk perception, complying with the PA recommendation, and having positive intentions in terms of FV consumption (Table 5.2). 
Table 5.2: Linear regression results: predictors of the number of completed intervention modules

\begin{tabular}{|c|c|c|c|c|c|c|}
\hline & \multicolumn{2}{|l|}{ Model 1} & \multicolumn{2}{|l|}{ Model 2} & \multicolumn{2}{|l|}{ Model 3} \\
\hline & Coefficient* & $p$ & Coefficient* & $p$ & Coefficient* & $p$ \\
\hline Age & 0.06 & .34 & 0.08 & .28 & 0.02 & .68 \\
\hline Gender & -0.09 & .13 & -0.05 & .47 & -0.03 & .63 \\
\hline Relationship & 0.14 & .02 & 0.15 & .02 & 0.14 & .02 \\
\hline Education & 0.01 & .86 & 0.05 & .47 & 0.04 & .50 \\
\hline Working situation & -0.09 & .18 & -0.11 & .10 & -0.08 & .19 \\
\hline Risk perception & & & 0.07 & .27 & 0.13 & .04 \\
\hline Recommendation PA & & & 0.20 & .01 & 0.18 & .01 \\
\hline Recommendation FV & & & -0.04 & .51 & -0.08 & .21 \\
\hline Outcome expectancies pos. PA & & & -0.08 & .23 & -0.03 & .60 \\
\hline Outcome expectancies neg. PA & & & -0.10 & .19 & -0.06 & .35 \\
\hline Outcome expectancies pos. FV & & & 0.15 & .05 & 0.09 & .20 \\
\hline Outcome expectancies neg. FV & & & 0.00 & .99 & -0.01 & .95 \\
\hline Self-efficacy PA & & & 0.02 & .77 & -0.01 & .93 \\
\hline Self-efficacy FV & & & -0.11 & .16 & -0.10 & .17 \\
\hline Intention FV & & & & & 0.46 & $<.001$ \\
\hline Intention (PA intensive) & & & & & -0.01 & .88 \\
\hline Intention (PA moderate) & & & & & 0.06 & .33 \\
\hline$R^{2}$ & .055 & & .123 & & .323 & \\
\hline
\end{tabular}

*standardised beta

Next, we analysed the predictors of generating APs (Table 5.3). The third model (using all predictors of the stepwise logistic regression analysis) indicated that participants who were more physically active at the baseline measurement generated significantly less APs concerning PA.

Additionally, the results of the third model in Table 5.3 for FV consumption indicated high risk perception and a strong intention to eat five portions of FVs daily as significant predictors of the generation of APs for this behaviour. 


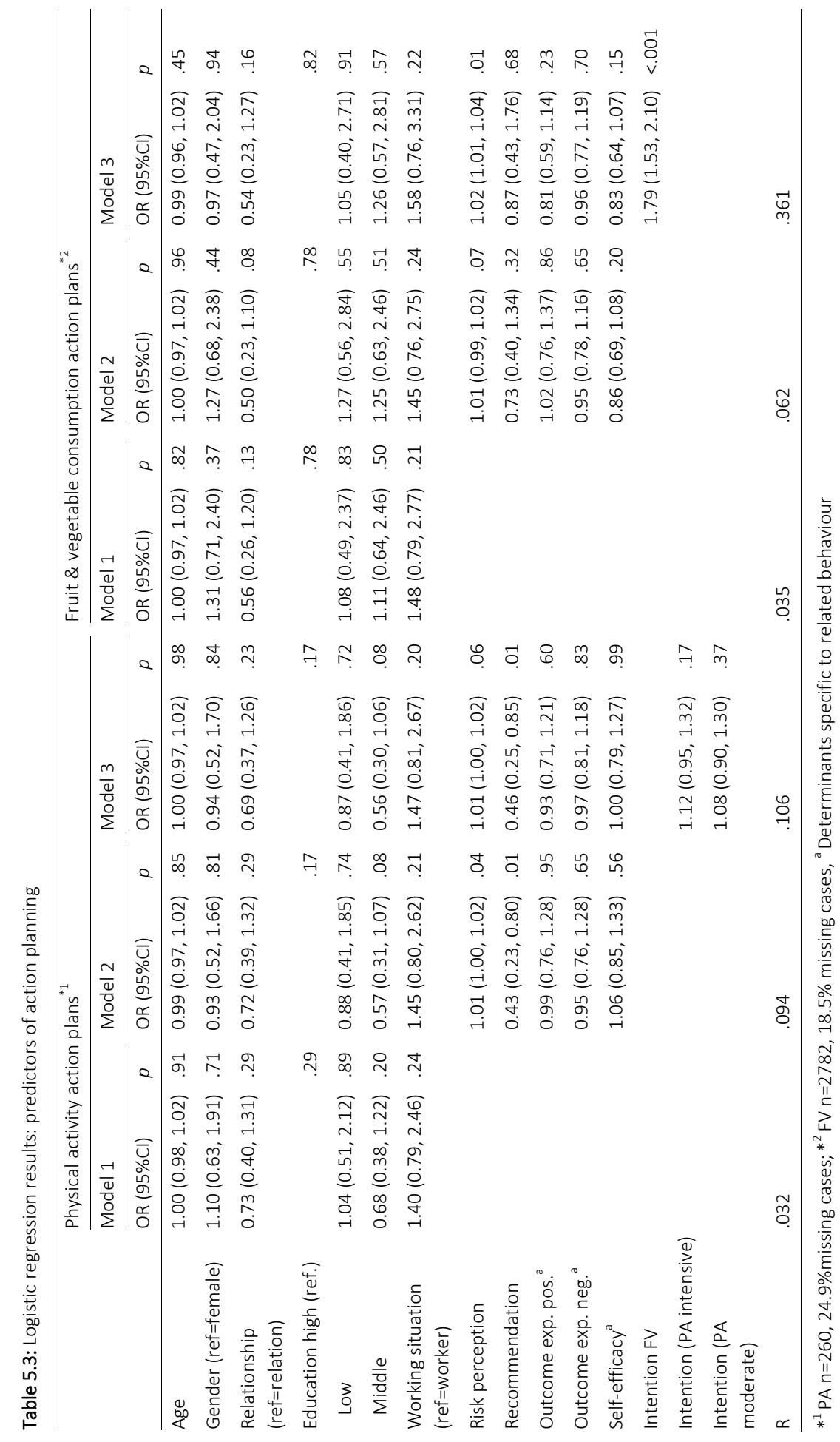




\section{Action plan quality}

The majority of the APs for PA ( $n=277,96.9 \%)$ and all APs regarding

FV consumption ( $n=139,100 \%$ ) were considered instrumental (i.e., they provided a goal-directed, reasonable action). More than half of the APs for PA (69.68\%) and for FV intake $(59.71 \%)$ were found to be highly specific (providing a detailed description of the plan). Table 5.4 shows the results for both APs for each behaviour. Furthermore, as Table 5.5 shows, most plans were evaluated by the participants as useful when using the "PEPP-rule".

A linear regression analysis (Table 5.6) indicated that being in a relationship is a significant predictor of AP specificity regarding PA.

Table 5.4: Action plan specificity for PA and FV consumption

\begin{tabular}{llllll}
\hline & \multicolumn{2}{l}{ Specificity action plan PA n (\%) } & & \multicolumn{3}{l}{ Specificity action plan FV n (\%) } \\
\cline { 2 - 3 } & AP 1 & AP 2 & & AP 1 & AP 2 \\
\hline Not specific & $3(2.0)$ & $7(5.1)$ & & $3(4.0)$ & $5(7.5)$ \\
Medium specific & $41(27.0)$ & $44(32.4)$ & & $26(34.7)$ & $25(37.3)$ \\
Highly specific & $108(71.0)$ & $85(62.5)$ & & $46(61.3)$ & $37(55.2)$ \\
\hline
\end{tabular}


Chapter 5

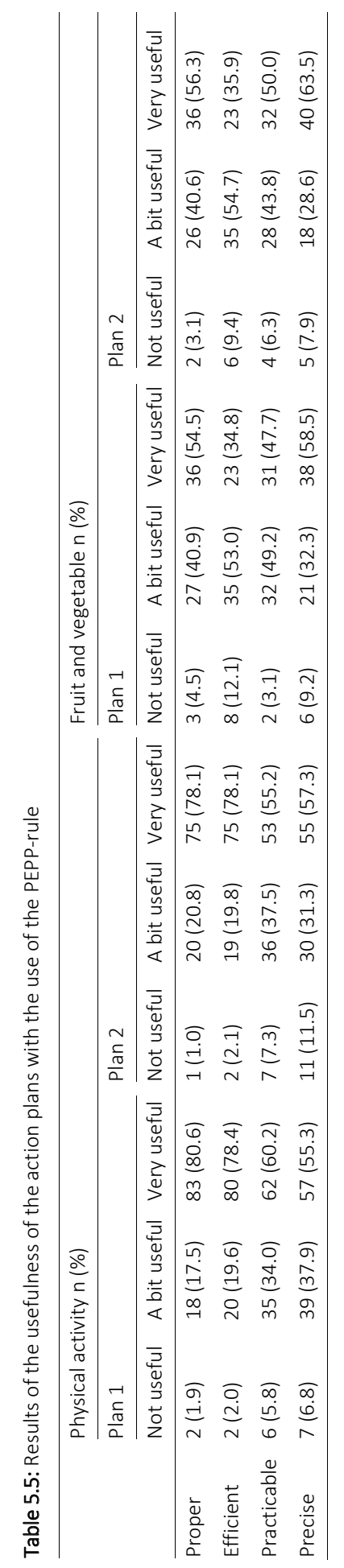


Generating and predicting high quality action plans

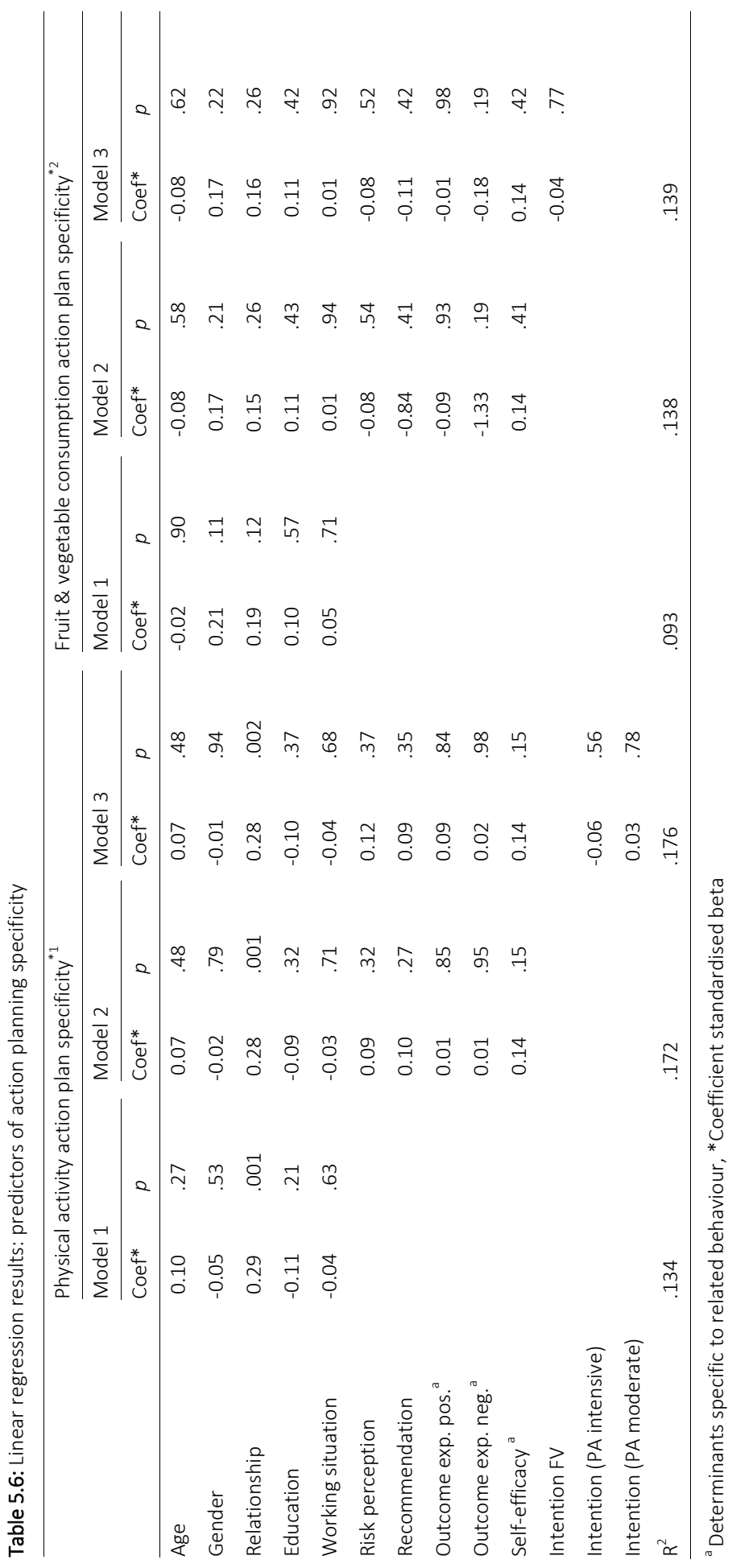




\section{DISCUSSION}

\section{Main findings}

\section{Use of intervention}

The purpose of this study was to evaluate the usage, generation and quality of APs concerning physical activity and fruit and vegetable consumption within a Web-based computer tailored intervention. This study shows that the intervention modules are used scarcely, that less than half of the participants generated APs, and that the quality of these APs was high. Another study that made use of action planning modules by which participants were required to generate their own APs also reported a low level of participation (van Genugten et al., 2014). A known shortcoming of Web-based intervention is the low level of usage (Eysenbach, 2005). The reason for "non-usage attrition" can vary in terms of interventional factors, demographic characteristics (Reinwand, Crutzen, et al., 2015), and social-cognitive causes (Christensen et al., 2009; Me Neve, Collins, \& Morgan, 2010; Todkill \& Powell, 2013). With regards to demographic characteristics, we only found that participants who were in a relationship made more use of the intervention. While people who are in a relationship have typically healthier lifestyles (Umberson \& Montez, 2010) and report a better perceived health (Kiecolt-Glaser \& Newton, 2001), it is also known that individuals in a relationship are less physically active and have a higher body weight than single people (Umberson, Crosnoe, \& Reczek, 2010). This could explain why people who are in a relationship have a high interest in using the intervention to improve their PA.

Furthermore, we found that participants who have a high risk perception with regards to cardiovascular diseases and those who have a strong intention to change their FV consumption behaviour made more use of the intervention modules. This corresponds with the findings that risk perception (Brewer et al., 2007) and intention (Sheeran, 2002) are positively related to a change in health behaviour. People with a high level of risk perception tend also to seek more information (ter Huurne \& Gutteling, 2008). This might suggest that those participants who have high risk perception used the intervention to gain more information on how to change their health behaviours, which might also be true for highly motivated participants.

Participants at baseline who complied with the PA recommendation made more use of the intervention and this may be due to the positive feedback that they received during the intervention due to their behaviour. Participants who complied with the behavioural recommendation at the start of the intervention got positive feedback and received less information about how to change their behaviour. For those participants, the intervention might be less time-consuming in contrast to participants who did not comply with the recommendation. They received more feedback and were asked to change their behaviour. If people need to process a lot of new information, this requires a lot of resources. This might decrease their self-regulatory capacity which is called ego 
depletion (Baumeister et al., 1994; Baumeister et al., 2000) and could hinder participants from participating in all single intervention modules.

\section{Generation of action plans}

Those participants who were more physically active at baseline were less likely to generate APs for PA. It might be that participants who were already active skipped the application of the AP because they have more experiences about when, where, and how to be physically active. Furthermore, it is reasonable that less physically active participants made more use of the AP modules because they received more tailored feedback on their behaviour and how to generate APs, which might have encouraged them to use the application more.

We have found that participants who had a high risk perception generated more APs for FV consumption. As mentioned above, those participants might be more interested in relevant information about how to change their health behaviour (ter Huurne \& Gutteling, 2008) and might therefore generate APs.

Furthermore, having a strong intention to increase one's FV consumption was found to be a significant predictor of generating APs for that behaviour. Since intention is one of the most important determinants of behavioural change (Sheeran, 2002) and a parameter of use with regards to optimising the effectiveness of APs (Kok et al., 2015), it seems plausible that participants who had a strong intention to change made the effort to generate APs to increase their self-regulation skills in terms of performing this behaviour in practice (van Osch, Reubsaet, Lechner, \& de Vries, 2008).

\section{Action plan quality}

We found that nearly all APs were highly instrumental, indicating that they were goaldirected. It is reasonable to assume that only participants who had a high intention to change their behaviours used this intervention module and made serious efforts to generate APs. In addition, more than half of the plans were highly specific and provided a high amount of details about what, where, when, how, and with whom the plan will be performed. APs that were medium-specific did not mention all details of the defined AP. This is in line with another study about the quality of action planning that reported that less specific plans did not mention the time at which the action would be performed (van Genugten et al., 2014). Nevertheless, the good quality of the plans could be explained by the fact that participants were guided through the planning modules by providing examples of effective plans, by asking participants to fill in their plans step-by-step, and because participants needed to evaluate their APs using the "PEPP-rule". This practical application seems to be useful in terms of generating high quality APs. 
Regarding predictors of plan specificity, we found that being in a relationship has a positive influence on the quality of APs. It is conceivable that this is biased with the presence of the partners while generating the AP and participants filled in the "with whom" section with their partner, which resulted in a higher plan specificity as defined.

Contrary to our assumption that participants that have different socio-demographic characteristics might use the intervention modules differently (Reinwand, Crutzen, et al., 2015) or might generate different plans with regards to plan quality, no such difference could be found. This might imply that the "PEPP-rule" application is equally effective for people who have different educational levels or different working situations to generate instrumental and specific APs.

\section{Strengths, limitations, and recommendations}

One of the strengths of the study is that we were able demonstrate in our Web-based tailored computer intervention that not only did participants generate APs but that they were generally of high quality. We distinguished between instrumentality and specificity. AP quality had found little attention in the existent literature. This study adds to previous research that the "PEPP-rule" can provide a valuable means to generate instrumental and specific APs in an eHealth intervention. The results of this study provide further insight as to how usage of intervention modules depends on sociodemographic variables and social cognitive variables. A further strength of our results is that the generated APs were part of an intervention and were not gained from a clinical setting. This might allow generalisation of the results to the field of Web-based interventions, thereby expanding the insights into previous studies that were carried out in more controlled settings.

There are also limitations of our study. The first limitation is that intervention use decreased rapidly from module to module and that only a minority of participants generated APs regarding FV consumption. This could be caused by the given sequence of the intervention. Participants had no choice as to which module they preferred to improve and neither did participants have the opportunity to choose with which behaviour they preferred to start. Therefore, it could be possible that participants who were interested in changing their FV consumption did not participate in certain intervention modules because they had no interest in completing the modules about PA. It is not yet known whether it is advisable to give participants the control to choose what to do within an intervention or to guide participants through an intervention. Participants who were guided through a website and had less control about what to do remembered more information afterwards (Crutzen, Cyr, \& de Vries, 2012), while a study found that participants who had no choices in an online intervention dropped out more often (D. N. Schulz et al., 2012). Further studies might want to find a balance between guiding participants through an intervention while also providing individual choices. 
Secondly, we did not find any predictors for AP specificity for FV consumption. This could have been caused by the small amount of participants that generated APs for this behaviour.

Finally, within the study, we tried to optimise the scope of the intervention by recruiting participants via different channels such as advertisements in hospitals, in newspapers, and on social media networks; and this study might, therefore, be vulnerable to selection bias (Reinwand et al., 2013). We assumed that mainly people with a strong intention to change their health behaviour would register themselves for participation. Therefore, our intervention was mainly focused on participants within the volitional phase of behaviour change. For participants who did not develop the intention to change their behaviour, the intervention was perhaps less suitable. The focus on action planning can only result in behavioural change if participants have a positive intention. Further interventions should add modules for participants in the motivational phase to prevent non-usage attrition (Hagger \& Luszczynska, 2014).

\section{CONCLUSION}

While our intervention was scarcely used, the generated APs were of a high quality. The generated APs were highly instrumental and specific. Providing a guided step-by step application with the opportunity to adjust APs by using the "PEPP-rule" seems to be a promising application to formulate APs for highly motivated participants who wish to improve their PA and FV consumption.

\section{Competing interests}

$\mathrm{HdV}$ is the scientific director of Vision2Health, a company that licenses evidence-based innovative computer-tailored health communication tools. The other authors declare that they have no competing interests.

\section{Authors' contributions}

SL developed the study concept and aims. TK, JW, DR designed the intervention and collected the data. SL and HdV provided intellectual input for the development of the questionnaire and intervention. DR drafted the manuscript and together with RC coded the action plans. Furthermore, DR, RC, VS, JW, TK, SL, and HdV provided extensive feedback on the manuscript. All authors read and approved the final manuscript. 


\section{ACKNOWLEDGEMENTS}

This project is funded through a grant by "Wilhelm-Stiftung für Rehabilitationsforschung, im Stifterverband für die deutsche Wissenschaft", a German foundation for rehabilitation research.

\section{AVAILABILITY OF DATA AND MATERIALS}

The data which was used in the current study is available and can be obtained from the authors. We have uploaded the dataset on Open Science Framework. 


\section{ABSTRACT}

Background: Web-based computer-tailored interventions have shown to be effective in improving health behavior; however, high dropout attrition is a major issue in these interventions.

Objective: The aim of this study is to assess whether people with a lower educational level drop out from studies more frequently compared to people with a higher educational level and to what extent this depends on evaluation of these interventions.

Method: Data from seven randomized controlled trials of Web-based computer-tailored interventions were used to investigate dropout rates among participants with different educational levels. To be able to compare higher and lower educated participants, intervention evaluation was assessed by pooling data from these studies. Logistic regression analysis was used to assess whether intervention evaluation predicted dropout at follow-up measurements.

Results: In three studies, we found a higher study dropout attrition rate among participants with a lower educational level, whereas in two studies we found that middle educated participants had a higher dropout attrition rate compared to highly educated participants. In four studies, no such significant difference was found. Three of seven studies showed that participants with a lower or middle educational level evaluated the interventions significantly better than highly educated participants (Alcohol-Everything within the Limit: $F_{(2,376)}=5.97, p=.003$; My Healthy Behavior: $F_{(2,359)}=5.52, p=.004$; Master Your Breath: $\left.F_{(2,317)}=3.17, p=.04\right)$. One study found lower intervention evaluation by lower educated participants compared to participants with a middle educational level (Weight in Balance: $F_{(2,37)}=3.17, p=.05$ ). Low evaluation of the interventions was not a significant predictor of dropout at a later follow-up measurement in any of the studies.

Conclusion: Dropout attrition rates were higher among participants with a lower or middle educational level compared with highly educated participants. Although lower educated participants evaluated the interventions better in approximately half of the studies, evaluation did not predict dropout attrition. Further research is needed to find other explanations for high dropout rates among lower educated participants.

Keywords: dropout; attrition; educational level; computer tailoring; Web-based intervention; eHealth; evaluation; meta-analysis 


\section{INTRODUCTION}

Previous studies have demonstrated that Web-based computer-tailored interventions can be effective in motivating individuals to adopt different health behaviors (S. Noar \& Harrington, 2012; S. M. Noar et al., 2011; Wantland, Portillo, Holzemer, Slaughter, \& McGhee, 2004), such as increasing physical activity (Ammann et al., 2013; Compernolle, Vandelanotte, Cardon, De Bourdeaudhuij, \& De Cocker, 2015; Konstantinidis et al., 2014; G. J. Norman et al., 2007; Peels et al., 2014; van 't Riet et al., 2010; van Stralen et al., 2011), improving healthy nutrition (Brug et al., 1999; Hamel \& Robbins, 2013; Krebs et al., 2010; Kroeze et al., 2006), smoking cessation (Elfeddali, Bolman, Candel, et al., 2012; McClure et al., 2013; Smit et al., 2012), reducing alcohol intake (Boon, Risselada, Huiberts, Riper, \& Smit, 2011; D. N. Schulz et al., 2013; van der Wulp et al., 2014), and have been successfully applied to multiple health behaviors (Smeets et al., 2007; van Keulen et al., 2011). In addition, these interventions have been found to be more costeffective than usual care or non-tailored information (D. N. Schulz, Smit, et al., 2014; Smit et al., 2013; Stanczyk, Bolman, Smit, et al., 2014; van Keulen et al., 2010).

According to Eysenbach (Eysenbach, 2005), dropout, either not completing the study or missing follow-up measurements, is a "fundamental characteristic" of Internet interventions and a problematic issue. The loss of participants to follow-up, dropout attrition, makes analyses and statements of the effectiveness of these interventions more complicated and less valid (Eysenbach, 2005), because most outcome measures are assessed during follow-up and dropout from the intervention seems to be related to dropout attrition (Cunningham, 2014). Therefore, it is important to find out why participants do not complete Web-based studies to ultimately understand and reduce this problem.

Acquiring follow-up measurements from at-risk groups, such as people with a lower educational level, is important because unhealthy lifestyle behaviors are most common among people with a lower educational level (Cowell, 2006; Margolis, 2013; Vlismas et al., 2009). Lower educated people are known to eat less fruits and vegetables (De IralaEstevez et al., 2000; Hulshof et al., 2003), are less physically active (Gidlow et al., 2006; Vlismas et al., 2009), consume alcohol more often in unhealthy quantities (Groot \& Maassen van den Brink, 2006), use more tobacco (Grittner, Kuntsche, Gmel, \& Bloomfield, 2013; Hiscock et al., 2012; Savage, 2012), and have a higher likelihood of being obese (Cutler \& Lleras-Muney, 2010) compared to highly educated people. It is not only important to reach this group with Web-based computer-tailored interventions, but also to prevent lower educated participants from dropping out of the follow-up measurements to be able to collect information about the effectiveness of the intervention (Cunningham, 2014).

Christensen and Mackinnon (Christensen \& Mackinnon, 2006) already raised the issue of insufficient research regarding study dropout in 2006. Since then, findings about dropout among participants with different educational levels are still rarely 
reported and show ambiguous results. Although some studies revealed that people with a lower educational level have higher dropout rates in Web-based computer-tailored interventions than highly educated people (Geraghty et al., 2012; E. Murray et al., 2013; Price et al., 2012; Rogers, 2003; Strecher et al., 2008), other studies did not find educational differences in terms of dropout (Ezendam et al., 2012; Habibović et al., 2014; E. Murray et al., 2013; Peel et al., 2012). To the best of our knowledge, no study indicates a significant higher dropout among highly educated participants, but there remains a need to obtain more insight into dropout among people with different educational levels to be able to reduce dropout.

The reason for dropout among lower educated people is rarely discussed in the literature. Possible reasons for dropout can be intervention characteristics (e.g., workload, content), personal characteristics such as educational level, or it can be related to participants' perceptions of the interventions, such as a lack of perceived benefit, which may result in dissatisfaction (Kelders et al., 2012). Dissatisfaction with the intervention can be reflected in the evaluation of the intervention. It has been shown that participants who do evaluate the intervention as less positive are more likely to drop out (Postel et al., 2011) and, therefore, might not be interested in attending follow-up measurements. In other words, evaluation might be a predictor of dropout attrition in Web-based computer-tailored interventions, but little thought has been given to this aspect, which makes it difficult to draw valid conclusions (Skelton, Irby, \& Geiger, 2014).

Therefore, the aim of this study is first to examine if the dropout attrition rates in our seven randomized controlled trials (RCTs) of Web-based computer-tailored interventions were higher for people with a lower educational level than people with a middle or high educational level. Second, we assessed whether people with different educational levels evaluated these interventions differently. Finally, we analyzed whether participations' evaluation of the interventions predicted dropout at subsequent follow-up measurements.

\section{METHODS}

\section{Studies}

To examine differences in dropout attrition and evaluation between participants with different educational levels, we used a convenience sample of participants from seven Web-based computer-tailored intervention studies that were conducted in the past years (2010-2014) at the Department of Health Promotion of Maastricht University in the Netherlands.

The studies were RCTs to evaluate interventions that used computer-tailored techniques to improve diverse health behaviors. The study "Master Your Breath" (MYB) 
focused on increasing physical activity and smoking cessation among chronic obstructive pulmonary disease (COPD) patients and people at risk for COPD. The three studies "Stay Quit for You" (SQ4U), "Support to Quit" (STQ), and "Personal Advice in Stopping smoking" (PAS) focused on smoking cessation. "Weight in Balance" (WIB) aimed to prevent obesity by targeting physical activity and energy intake. The study "My Healthy Behavior" (MHB) targeted the following health behaviors: physical activity, fruit and vegetable consumption, alcohol intake, and smoking. The study "Alcohol-Everything within the Limits" (AEL) focused on moderate alcohol intake and is the only study that was not carried out in the Netherlands but in Germany.

All selected studies made use of the I-Change model (de Vries et al., 2003; van der Wulp, Hoving, \& de Vries, 2013). The I-Change model postulates that the behavior change process has at least three phases: awareness, motivation, and action. The first factor is determined by factors such as behavioral awareness, knowledge, and risk perceptions. The second phase is determined by attitudes, social influence beliefs, and self-efficacy expectations and results in a certain intention to perform a particular behavior. The third factor is determined by self-efficacy, action planning, skills, and barriers. The tailored feedback messages of the studies included in this paper have a strong focus on inter alia these determinants. A detailed description of these RCTs and the related publications can be found in Table 6.1. 


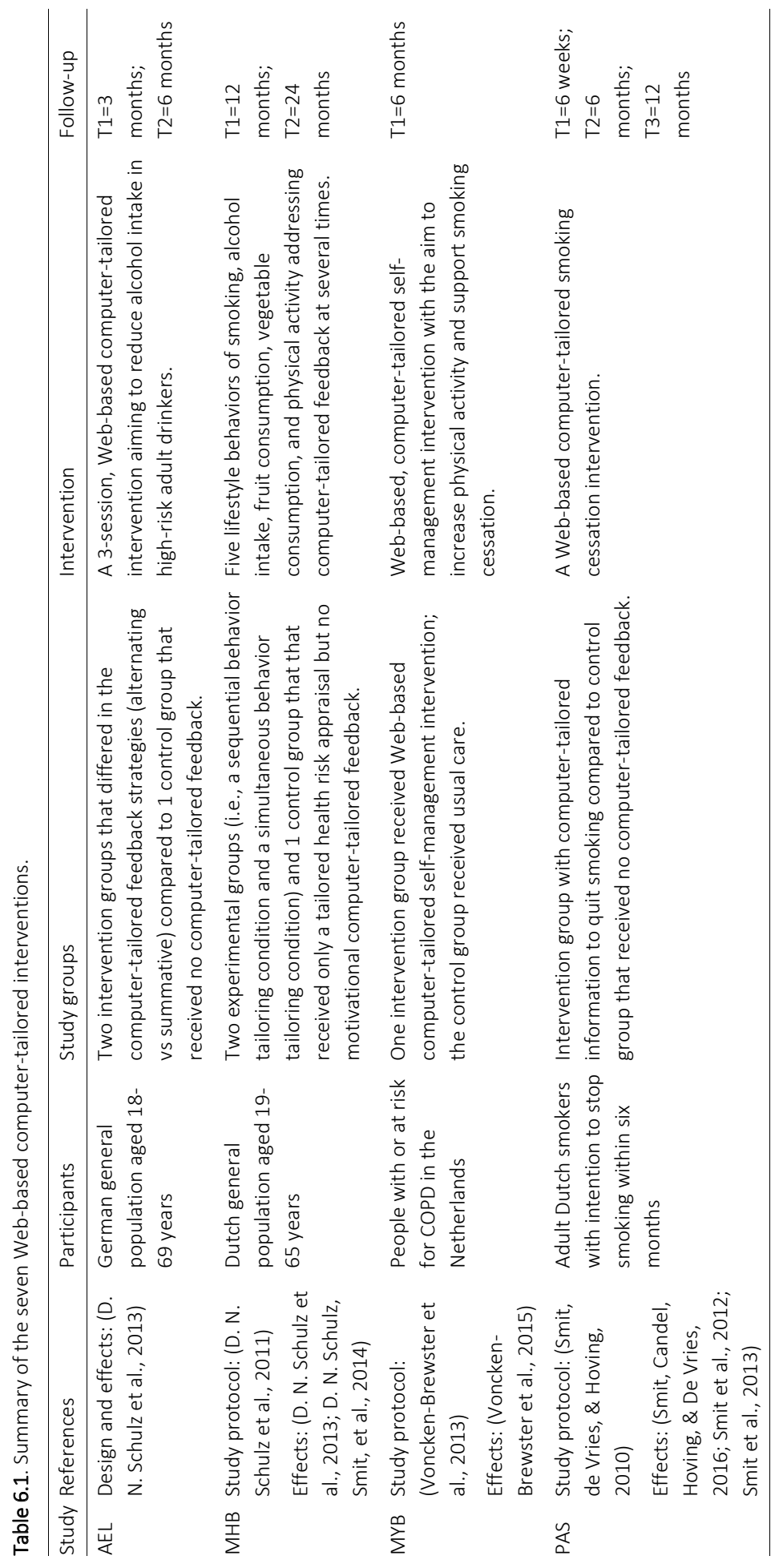




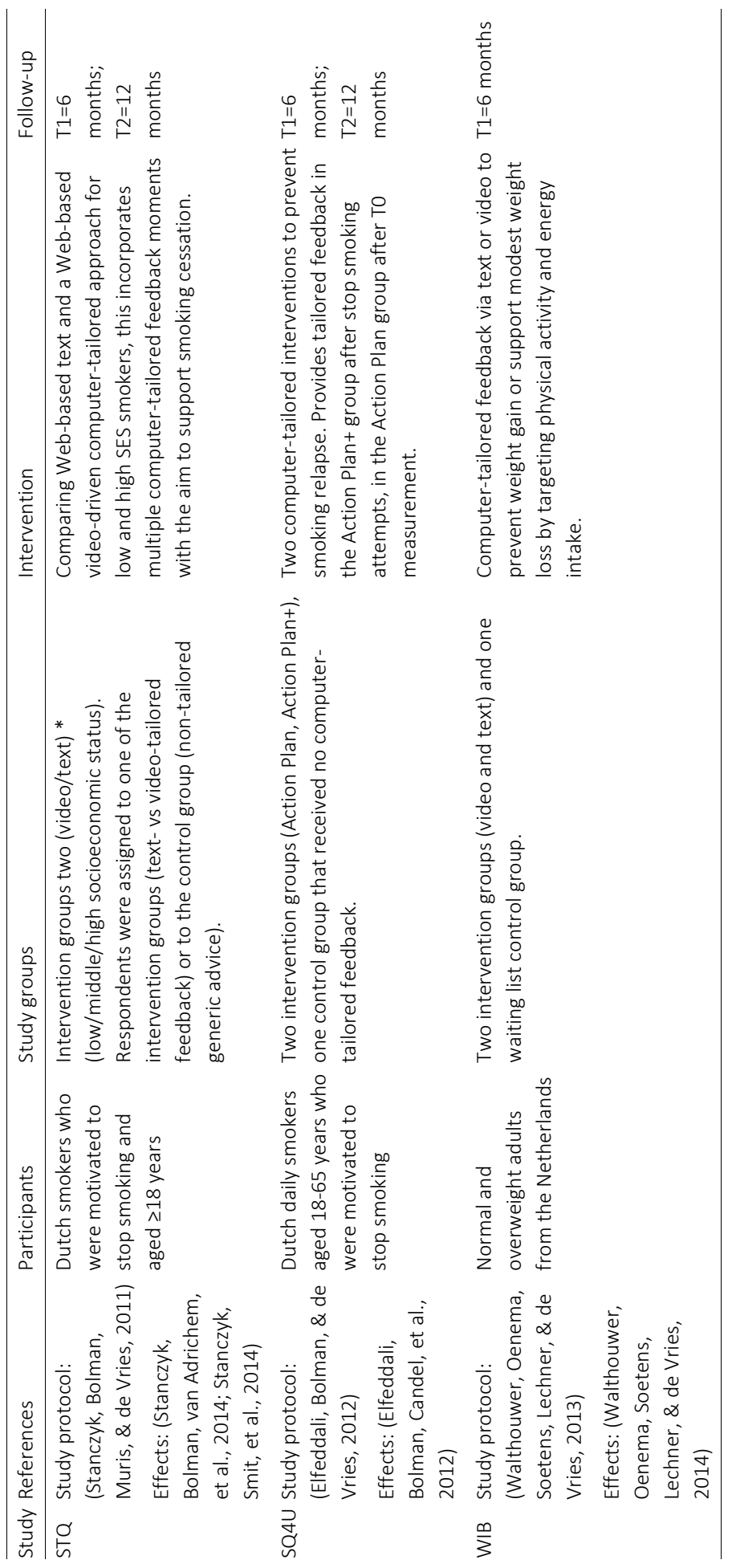




\section{Measurement}

In all seven studies, educational level was assessed by asking participants about their highest completed level of education. In-line with national guidelines, educational level was categorized into three groups: lower (1=no education, primary or lower vocational school), middle (2=secondary vocational school or high school), and higher (3=higher professional education or university) educational level (Luijkx \& de Heus, 2008).

All studies included a process evaluation assessment to evaluate the intervention among participants within the intervention group. Participants were asked to evaluate the tailored feedback and the intervention. The process evaluation assessments of the seven studies included had one item in common that asked participants to grade the intervention that they participated in: "Please evaluate the intervention with a school grade from 1 to 10" (10=highest grade, 1=lowest grade according to the Dutch school grading system; AEL: 15=highest grade, 1=lowest grade, which is in-line with the German school grading system).

To assess dropout attrition, participants who completed the baseline measurement but did not complete the follow-up measurement were characterized as dropouts (1=dropout; $0=$ completed follow-up). We assessed dropout attrition within differently educated participants for each follow-up measurement separately. Furthermore, we used the last available evaluation moment as predictor of dropout for the following measurement. Table 6.1 gives an overview of the specific follow-up moments per study.

\section{Statistical Analysis}

All analyses were performed with SPSS 20.0 (IBM Corp, Armonk, NY, USA). Descriptive statistics were used to describe sample characteristics. Per study, a logistic regression analysis was conducted to examine if dropout rates differed per educational level. To control for multiple testing, the Benjamini and Hochberg linear step-up method was used for each study (Benjamini \& Hochberg, 1995; Benjamini \& Yekutieli, 2001). With the use of an Excel template, the adjusted significance levels were calculated (Weinkauf, 2012).

Differences between the educational levels with regard to evaluation of the Webbased computer-tailored interventions were analyzed by means of ANOVAs and Tukey honestly significant difference (HSD) tests. Control groups were excluded from analysis with regard to evaluation of the intervention because they could not evaluate it.

To be able to give a more general picture of whether lower and higher educated participants from the intervention groups evaluated Web-based computer-tailored interventions differently, the Exploratory Software for Confidence Intervals (ESCI) Excel template (Cumming, 2011) was used for pooling the data by means of a meta-analysis (Table 6.1). The meta-analysis used a random-effect model and gave an impression of the overall differences for intervention evaluation between lower and higher educated 
participants (i.e., by subtracting the evaluation of the most different groups, the lower educated participants from the higher educated participants). In one study (MHB), the evaluation item was assessed at multiple follow-up measurements; in this case, we included only the last follow-up measurement (Borenstein, Hedges, Higgins, \& Rothstein, 2009).

Finally, logistic regression analyses were conducted to examine if dropout was predicted by evaluation in four of the seven interventions among participants with different educational levels. We excluded the studies WIB, PAS, and MYB from this analysis because their evaluation assessment took place during the last follow-up measurement; therefore, it is not possible to assess evaluation as a predictor of dropout in these studies. To identify possible interaction effects, an interaction term of educational level and evaluation was used in the regression model. If this interaction term was significant, then analyses were conducted separately per educational level. Analyses were corrected for age and gender. A $p$-value of .05 was used as the significance level for all analysis.

\section{RESULTS}

\section{Participants}

Table 6.2 shows the educational level, mean age, and gender distribution of the participants within the seven selected studies at baseline.

Table 6.2. Baseline sample characteristics of the participants in the seven Web-based computer-tailored interventions.

\begin{tabular}{|c|c|c|c|c|c|c|}
\hline \multirow[t]{2}{*}{ Study } & \multirow[t]{2}{*}{$\mathrm{N}$} & \multicolumn{3}{|c|}{ Educational level, n (\%) } & \multirow{2}{*}{$\begin{array}{l}\text { Age (years), } \\
\text { mean (SD) }\end{array}$} & \multirow{2}{*}{$\begin{array}{l}\text { Gender (male), } \\
\mathrm{n}(\%)\end{array}$} \\
\hline & & Low & Middle & High & & \\
\hline AEL & 1149 & $483(44.8)$ & $256(23.8)$ & $338(31.4)$ & $43.82(15.51)$ & $550(47.9)$ \\
\hline $\mathrm{MHB}$ & 5055 & $515(10.4)$ & $2334(47.05)$ & $2112(42.57)$ & 44.15 (12.67) & 2661 (52.64) \\
\hline MYB & 1307 & $386(29.5)$ & $427(32.7)$ & $494(37.8)$ & $57.64(7.22)$ & 627 (47.9) \\
\hline PAS & 1123 & $238(21.2)$ & $513(45.7)$ & $372(33.1)$ & 49.47 (32.55) & $535(47.6)$ \\
\hline STQ & 2099 & 707 (33.6) & $782(37.3)$ & $612(29.2)$ & $45.33(13.21)$ & 821 (39.1) \\
\hline SQ4U & 2031 & $207(10.2)$ & $1130(55.64)$ & $694(34.2)$ & $40.88(11.80)$ & 766 (37.7) \\
\hline WIB & 1419 & $214(15.1)$ & $436(30.73)$ & $769(54.2)$ & $48.13(11.52)$ & $588(41.4)$ \\
\hline
\end{tabular}

${ }^{a}$ For reference, the average educational level in Germany for low, middle, and high is 39, 22, and 27, respectively (Statistisches \& Bundesamt, 2013); for the Netherlands, it is 30, 28, and 42, respectively (Bierings, 2013). 
Chapter 6

Dropout

Table 6.3 shows the results of the dropout analyses with regard to the educational level for each study, each follow-up measurement including the dropout rates, and study group in detail with high education as the reference group.

Table 6.3. Results of a logistic regression examine dropout attrition among different educational groups.

\begin{tabular}{|c|c|c|c|c|c|}
\hline \multirow[t]{3}{*}{ Study, group and follow-up } & \multirow[t]{3}{*}{ Dropout n (\%) } & \multicolumn{4}{|l|}{ Educational level $^{a}$} \\
\hline & & \multicolumn{2}{|l|}{ Low } & \multicolumn{2}{|l|}{ Middle } \\
\hline & & OR $(95 \% \mathrm{Cl})$ & $p$ & OR $(95 \% \mathrm{Cl})$ & $p$ \\
\hline \multicolumn{6}{|l|}{$A E L$} \\
\hline $\mathrm{T} 1$ & $398(34.6)$ & & & & \\
\hline Sequential & & $0.61(0.10,3.59)$ & .58 & $1.03(0.15,7.18)$ & .97 \\
\hline Simultaneously & & $1.65(0.61,4.58)$ & .32 & $-^{\mathrm{c}}$ & .99 \\
\hline Control & & $-^{c}$ & .99 & $-^{c}$ & .99 \\
\hline $\mathrm{T} 2$ & $436(37.9)$ & & & & \\
\hline Sequential & & $1.16(0.24,5.52)$ & .89 & $1.90(0.18,19.37)$ & .58 \\
\hline Simultaneously & & $1.15(0.34,3.84)$ & .81 & $1.23(0.21,7.13)$ & .81 \\
\hline Control & & $0.90(0.11,7.06)$ & .92 & $0.51(0.37,7.09)$ & .61 \\
\hline \multicolumn{6}{|l|}{$\mathrm{MHB}$} \\
\hline $\mathrm{T} 1$ & 3317 (65.62) & & & & \\
\hline Sequential & & $1.52(1.14,2.01)$ & $.004^{b}$ & $1.05(0.83,1.32)$ & .68 \\
\hline Simultaneously & & $1.57(1.18,2.08)$ & $.002^{b}$ & $1.39(1.09,1.78)$ & $.007^{b}$ \\
\hline Control & & $1.32(1.00,1.73)$ & .04 & $1.27(1.00,1.61)$ & .04 \\
\hline $\mathrm{T} 2$ & $3602(71.26)$ & & & & \\
\hline Sequential & & $1.43(1.06,1.94)$ & $.01^{b}$ & $0.95(0.74,1.23)$ & .73 \\
\hline Simultaneously & & $1.51(1.12,2.04)$ & $.006^{b}$ & $1.50(1.16,1.94)$ & $.002^{b}$ \\
\hline Control & & $1.23(0.93,1.62)$ & .14 & $0.97(0.76,1.23)$ & .81 \\
\hline \multicolumn{6}{|l|}{ MYB } \\
\hline $\mathrm{T} 1$ & $254(19.4)$ & & & & \\
\hline Intervention & & $1.33(0.84,2.12)$ & .21 & $1.40(0.89,2.20)$ & .13 \\
\hline Control & & $1.14(0.67,1.95)$ & .61 & $1.17(0.70,1.96)$ & .53 \\
\hline \multicolumn{6}{|l|}{ PAS } \\
\hline $\mathrm{T} 1$ & $674(60.0)$ & & & & \\
\hline Control & & $0.93(0.58,1.49)$ & .77 & $0.88(0.59,1.31)$ & .55 \\
\hline Tailoring only & & $2.02(1.23,3.33)$ & .005 & $1.37(0.93,2.01)$ & .10 \\
\hline $\mathrm{T} 2$ & $831(74.0)$ & & & & \\
\hline Control & & $0.93(0.54,1.56)$ & .77 & $0.89(0.57,1.39)$ & .61 \\
\hline Tailoring only & & $2.04(1.15,3.60)$ & .01 & $1.41(0.93,2.15)$ & .10 \\
\hline
\end{tabular}




\begin{tabular}{|c|c|c|c|c|c|}
\hline \multirow[t]{3}{*}{ Study, group and follow-up } & \multirow[t]{3}{*}{ Dropout n (\%) } & \multicolumn{4}{|l|}{ Educational level $^{a}$} \\
\hline & & \multicolumn{2}{|l|}{ Low } & \multicolumn{2}{|l|}{ Middle } \\
\hline & & OR $(95 \% \mathrm{Cl})$ & $p$ & OR $(95 \% \mathrm{Cl})$ & $p$ \\
\hline T3 & $967(86.1)$ & & & & \\
\hline Control & & $1.42(0.71,2.86)$ & .32 & $0.89(0.52,1.52)$ & .67 \\
\hline Tailoring only & & $1.41(0.67,2.97)$ & .35 & $1.03(0.60,1.78)$ & .90 \\
\hline \multicolumn{6}{|l|}{ STQ } \\
\hline $\mathrm{T} 1$ & $1306(62.22)$ & & & & \\
\hline Video & & $1.90(1.24,2.90)$ & $.003^{b}$ & $1.39(0.93,2.09)$ & 10 \\
\hline Text & & $1.29(0.87,1.91)$ & .19 & $1.22(0.83,1.78)$ & .29 \\
\hline Control & & $0.98(0.67,1.47)$ & .98 & $0.71(0.49,1.05)$ & .09 \\
\hline $\mathrm{T} 2$ & $1437(68.46)$ & & & & \\
\hline Video & & $1.95(1.26,3.02)$ & $.003^{b}$ & $1.39(0.92,2.09)$ & .11 \\
\hline Text & & $2.31(1.52,3.51)$ &.$<001^{b}$ & $1.29(0.88,1.89)$ & .18 \\
\hline Control & & $1.36(0.90,2.04)$ & .13 & $1.24(0.84,1.84)$ & .66 \\
\hline \multicolumn{6}{|l|}{ SQ4U } \\
\hline $\mathrm{T} 1$ & $1251(61.93)$ & & & & \\
\hline Action Plan & & $1.26(0.75,2.12)$ & .36 & $1.03(0.74,1.44)$ & .83 \\
\hline Action Plan + & & $1.71(0.92,3.18)$ & .08 & $1.14(0.81,1.60)$ & .44 \\
\hline Control & & $1.73(0.91,3.27)$ & .09 & $0.90(0.63,1.28)$ & .57 \\
\hline $\mathrm{T} 2$ & $1465(72.13)$ & & & & \\
\hline Action Plan & & $2.33(1.24,4.35)$ & .01 & $1.30(0.91,1.86)$ & .14 \\
\hline Action Plan + & & $2.25(1.11,4.52)$ & .02 & $1.55(1.07,2.24)$ & .01 \\
\hline Control & & $2.00(1.02,3.92)$ & .04 & $1.35(0.94,1.94)$ & .09 \\
\hline \multicolumn{6}{|l|}{ WIB } \\
\hline $\mathrm{T} 1$ & $404(28.5)$ & & & & \\
\hline Video & & $1.50(0.87,2.59)$ & .15 & $1.23(0.81,2.01)$ & .29 \\
\hline Text & & $2.29(1.33,3.95)$ & $.003^{b}$ & $1.12(0.74,1.69)$ & .60 \\
\hline Control & & $1.57(0.81,3.04)$ & .18 & $2.01(1.22,3.32)$ & $.006^{b}$ \\
\hline
\end{tabular}

\footnotetext{
${ }^{a}$ All analysis are corrected for age and gender. High education is the reference group.

${ }^{\mathrm{b}}$ Significant $p$-values after correction for multiple comparisons according to Benjamini-Hochberg.

${ }^{c}$ Odds ratios are not reported due to low cell count.
}

After correction for multiple testing, significantly higher dropout rates were found within three studies (MHB, STQ, WIB) among lower educated participants compared to higher educated ones. Furthermore, in these three studies, dropout attrition was also significantly higher among middle educated participants in comparison with higher educated participants. In four of seven studies (AEL, MYB, PAS, SQ4U), no difference in dropout with regard to educational level was found. 


\section{Chapter 6}

\section{Evaluation}

Table 6.4 presents differences between the educational groups with regard to evaluation of the Web-based computer-tailored interventions in detail. In three of seven studies (AEL, MHB, MYB), lower educated participants evaluated the intervention significantly higher compared to their counterparts. In one study (WIB), lower educated participants evaluated the intervention less positively compared to middle educated participants.

Table 6.4. Evaluation of the seven Web-based computer-tailored interventions by different educational levels.

\begin{tabular}{|c|c|c|c|c|c|c|c|c|}
\hline \multirow[t]{2}{*}{ Study and group ${ }^{a}$} & \multicolumn{3}{|c|}{ Level of education, mean (SD) } & \multirow[t]{2}{*}{$F$} & \multirow[t]{2}{*}{$P$} & \multicolumn{3}{|c|}{ Tukey HSD, $p^{\text {b }}$} \\
\hline & Low & Middle & High & & & L-M & $\mathrm{L}-\mathrm{H}$ & $\mathrm{M}-\mathrm{H}$ \\
\hline \multicolumn{9}{|l|}{$A E L$} \\
\hline \multicolumn{9}{|l|}{ TO } \\
\hline Sequential & $11.20(3.48)$ & $11.12(3.00)$ & $10.72(3.62)$ & $0.71(2,340)$ & .56 & .97 & .47 & .70 \\
\hline Simultaneously & $11.75(3.37)$ & $11.49(2.85)$ & $10.40(3.61)$ & $5.97(2,376)$ & .003 & .82 & .002 & .05 \\
\hline \multicolumn{9}{|l|}{$\mathrm{T} 2$} \\
\hline Sequential & $11.32(4.27)$ & $11.27(3.69)$ & $10.39(4.33)$ & $0.58(2.229)$ & .56 & .77 & .89 & .53 \\
\hline Simultaneously & $11.09(4.28)$ & $11.53(3.30)$ & $10.81(3.89)$ & $1.26(2.246)$ & .56 & .99 & .29 & .48 \\
\hline \multicolumn{9}{|l|}{$\mathrm{MHB}$} \\
\hline \multicolumn{9}{|l|}{$\mathrm{T} 1$} \\
\hline Sequential & $7.43(1.08)$ & 7.14 (1.79) & $7.03(1.13)$ & $2.12(2.201)$ & .12 & .98 & .48 & .11 \\
\hline Simultaneously & $7.80(0.91)$ & $6.94(1.70)$ & $6.56(2.41)$ & $1.30(2.178)$ & .27 & .60 & .27 & .62 \\
\hline \multicolumn{9}{|l|}{$\mathrm{T} 2$} \\
\hline Sequential & $7.78(1.27)$ & $7.59(0.94)$ & $7.53(0.91)$ & $1.04(2.367)$ & .35 & .64 & .37 & .71 \\
\hline Simultaneously & $7.94(0.89)$ & $7.68(0.92)$ & $7.43(1.02)$ & $5.52(2.359)$ & .004 & .30 & .01 & .05 \\
\hline \multicolumn{9}{|l|}{ MYB } \\
\hline \multicolumn{9}{|l|}{$\mathrm{T} 1$} \\
\hline Intervention & $7.07(1.50)$ & $6.93(1.23)$ & $6.60(1.77)$ & $3.17(2.317)$ & .04 & .77 & .05 & .17 \\
\hline \multicolumn{9}{|l|}{ PAS } \\
\hline \multicolumn{9}{|l|}{ T3 } \\
\hline Tailoring only & $6.09(1.70)$ & $6.72(1.25)$ & $7.03(1.20)$ & $2.42(2.81)$ & .09 & .92 & .96 & .63 \\
\hline \multicolumn{9}{|l|}{ STQ } \\
\hline \multicolumn{9}{|l|}{$\mathrm{T} 2$} \\
\hline Video & $6.48(1.97)$ & $6.18(2.17)$ & $6.28(1.64)$ & $0.38(2.193)$ & .69 & .67 & .83 & .95 \\
\hline Text & $6.53(1.84)$ & $6.51(1.23)$ & $5.96(1.72)$ & $3.29(2.234)$ & .04 & .99 & .08 & .07 \\
\hline \multicolumn{9}{|l|}{ SQ4U } \\
\hline \multicolumn{9}{|l|}{$\mathrm{T} 1$} \\
\hline Action Plan & $6.56(1.74)$ & $6.63(1.41)$ & $6.20(1.60)$ & $1.29(2.134)$ & .28 & .98 & .79 & .25 \\
\hline Action Plan+ & $6.27(2.10)$ & 6.49 (1.69) & $6.51(1.27)$ & $0.10(2.108)$ & .90 & .97 & .85 & .99 \\
\hline
\end{tabular}




\begin{tabular}{|c|c|c|c|c|c|c|c|c|}
\hline \multirow[t]{2}{*}{ Study and group ${ }^{a}$} & \multicolumn{3}{|c|}{ Level of education, mean (SD) } & \multirow[t]{2}{*}{$F$} & \multirow[t]{2}{*}{$P$} & \multicolumn{3}{|c|}{ Tukey HSD, $p^{b}$} \\
\hline & Low & Middle & High & & & L-M & L-H & $\mathrm{M}-\mathrm{H}$ \\
\hline \multicolumn{9}{|l|}{$\overline{\text { WIB }}$} \\
\hline \multicolumn{9}{|l|}{$\mathrm{T} 1$} \\
\hline Video & $6.99(1.23)$ & $7.56(0.78)$ & $7.36(1.08)$ & $3.17(2.37)$ & .05 & .05 & .27 & .21 \\
\hline Text & $6.79(0.92)$ & $7.32(0.82)$ & $7.11(1.24)$ & $0.66(2.51)$ & .52 & .49 & .67 & .88 \\
\hline
\end{tabular}

Furthermore, the meta-analysis of the seven studies, comparing evaluation of lower and higher educated participants, indicated that participants with a lower educational level evaluated the interventions significantly more positively compared to highly educated participants (OR=0.28, 95\% Cl: -0.54 to $0.04, p<.001$ ) (see Figure 6.1). Nevertheless, the meta-analysis revealed presence of a moderate level of heterogeneity $\left(I^{2}=66.10 \%\right) \quad$ (Higgins \& Thompson, 2002; Huedo-Medina, Sánchez-Meca, MarínMartínez, \& Botella, 2006), which indicates variation across the studies.

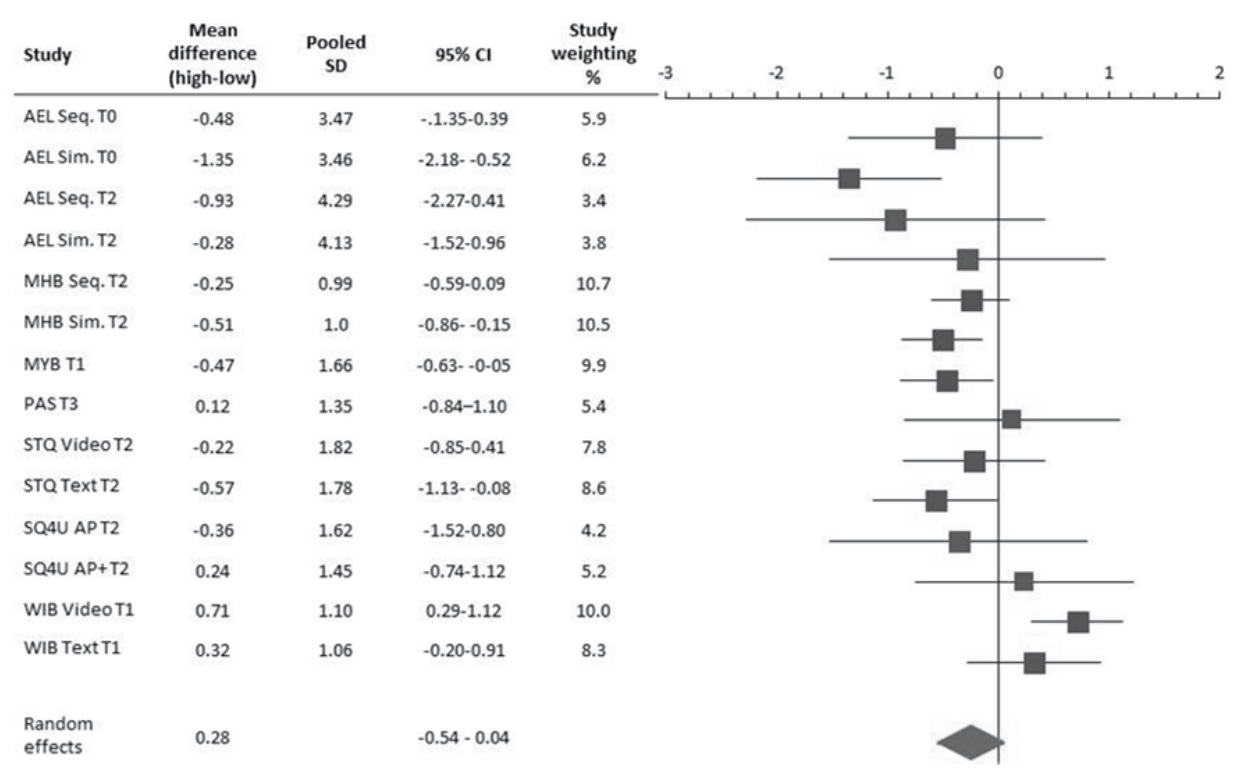

Figure 6.1. Forest plot of mean differences by random-effect model of evaluation of Web-based computertailored interventions between highly and lower educated participants. Random effects represents the combined effect. 


\section{Association of Education and Evaluation with Dropout Attrition}

For the four studies that evaluated the intervention before the follow-up measurements, a significant interaction between education and evaluation regarding dropout at follow-up was not found (Table 6.5). Only within the MHB study was a positive association found between the intervention evaluation and educational level. Participants with a middle educational level were more likely to dropout than participants with a higher educational level.

Table 6.5. Association of education and evaluation with dropout attrition at follow-up.

\begin{tabular}{|c|c|c|c|c|c|}
\hline Study and variables & $\beta$ & $p$ & OR $(95 \% \mathrm{Cl})$ & $x_{7}^{2}$ & $R^{2}$ \\
\hline AEL T1 & & & & 9.0 & .054 \\
\hline Education low & -1.02 & .35 & $0.35(0.04,3.14)$ & & \\
\hline Education middle & -0.08 & .96 & $0.92(0.02,37.18)$ & & \\
\hline Evaluation TO & 0.03 & .69 & $1.03(0.88,1.19)$ & & \\
\hline Education $\times$ evaluation & & .48 & & & \\
\hline MHB T1 & & & & 96.9 & .053 \\
\hline Education low & 1.90 & .05 & $6.71(0.97,46.43)$ & & \\
\hline Education middle & 0.93 & .07 & $2.55(0.89,7.27)$ & & \\
\hline Evaluation T0 & -0.01 & .84 & $0.99(0.90,1.08)$ & & \\
\hline Education $\times$ evaluation & & .18 & & & \\
\hline STQ T2 & & & & 16.6 & .033 \\
\hline Education low & 0.62 & .29 & $1.86(0.57,6.00)$ & & \\
\hline Education middle & 0.28 & .64 & $1.32(0.40,4.35)$ & & \\
\hline Evaluation T1 & -0.49 & .52 & $0.95(0.81,1.10)$ & & \\
\hline Education $\times$ evaluation & & .99 & & & \\
\hline SQ4U T2 & & & & 5.6 & .051 \\
\hline Education low & 4.33 & .11 & $76.36(0.34,16.713)$ & & \\
\hline Education middle & 1.32 & .55 & $3.75(0.46,303.55)$ & & \\
\hline Evaluation T1 & 0.11 & .69 & $1.11(0.64,1.92)$ & & \\
\hline Education $\times$ evaluation & & .40 & & & \\
\hline
\end{tabular}

${ }^{\mathrm{a}} \mathrm{T}$ indicates follow-up; high education is reference group. All analyses are corrected for age and gender.

\section{DISCUSSION}

\section{Dropout Attrition}

The first aim of this study was to evaluate whether participants with a lower educational level have higher dropout attrition from Web-based computer-tailored studies than 
participants with a medium or high educational level. In three of these studies, lower and middle educated participants dropped out more frequently compared to higher educated participants.

A possible explanation for the higher dropout rates may be that lower educated participants tend to use written health information more often (Cotten \& Gupta, 2004) and spend less time online seeking health information (Jensen et al., 2010; Wangberg et al., 2008). It could be possible that they lose interest in the intervention sooner, which causes them to drop out of the study.

Also, the fact that lower educated people have an unhealthier lifestyle (Cowell, 2006; Margolis, 2013; Vlismas et al., 2009) might play a role in dropout. Due to tailoring, participants with an unhealthier lifestyle in multiple health behavior interventions received more recommendations to change their health behavior(s) and this has been found to decrease participants' motivation to change (Wilson et al., 2015).It might be possible that lower educated participants started the intervention with the aim to change their health behavior, but that receiving information about extensive required changes decreased their self-efficacy to be able to change (Crutzen \& Ruiter, 2015) and could subsequently have decreased their motivation to participate. Another explanation could be that lower educated participants might have been less likely to change their behavior and, thus, may have perceived the recommendations as less feasible, which caused them to drop out of the study (Christensen et al., 2009; Eysenbach, 2005; Postel, de Jong, de Haan, Becker, \& ter Huurne, 2010). This could have caused not only usage and nonusage attrition, but also dropout attrition because these two kinds of attrition seem to be related (E. Murray et al., 2013).

Moreover, lower educated people might be less familiar with Web-based computertailored interventions (Kraft \& Yardley, 2009; Neter \& Brainin, 2012) and that might result in lower confidence in the effectiveness of those interventions (i.e., lower perceived efficacy) and, in turn, could cause an increase in dropout (Geraghty, Wood, \& Hyland, 2010; McKay, Danaher, Seeley, Lichtenstein, \& Gau, 2008). Although these are reasons for nonusage attrition (not using the intervention), it seems convincing that this correlates with dropout attrition because participants who did not evaluate the intervention positively might have little interest in completing follow-up measurements (Postel et al., 2011).

All participants were asked to complete long questionnaires and received tailored feedback, which must be cognitively processed and requires intensive cognitive performance. Lower educated adults have been shown to have a lower level of health literacy (Kickbusch, 2001; Neter \& Brainin, 2012; Paasche-Orlow, Parker, Gazmararian, Nielsen-Bohlman, \& Rudd, 2005). Those people have more difficulties to process new information and this could cause ego depletion (Baumeister et al., 1994; Baumeister et al., 2000). Ego depletion may, in turn, reduce the willingness to participate any longer within the study. 
Some studies found that dropout attrition could be increased by sending reminders and prompts (De Leon, Fuentes, \& Cohen, 2014; Fry \& Neff, 2009; E. Murray et al., 2013; Wangberg et al., 2008). Further research is necessary to evaluate if this is also effective for people with different educational levels.

\section{Evaluation}

Against our initial expectation that lower educated participants might evaluate the interventions less positively, we found that lower educated participants evaluated the intervention in three of seven studies more positively compared to their higher educated counterparts. This might be explained by the finding that highly educated people make more use of the Internet as health information resource, whereas these interventions might be newer and more interesting for lower educated people. A review supported this assumption because it shows that people with a high educational level may make more intensive use of several sources (e.g., people form their social network, mass media, health professionals) to gain health-related information compared to lower educated people and they might read the received information more superficially (Bell, 2014). This might result in less elaboration of the messages and a lower evaluation regarding the novelty of the messages. Due to the use of several resources, highly educated participants also rely less on online information and have lower levels of trust in them, which may negatively influence their evaluation of the intervention (Diviani et al., 2015).

Evaluation was not a significant predictor of dropout at follow-up in any of our studies. This suggests that other factors must be important in explaining why participants did not return to the study for follow-up questionnaires. Dropout analysis performed within these studies has shown that a lower educational level, unhealthy lifestyle, low intention to change the behavior, and low self-efficacy were predictors of dropout (Elfeddali, Bolman, Candel, et al., 2012; Kalichman et al., 2006; D. N. Schulz et al., 2013).

\section{Limitations and Strengths}

First, the only item all studies had in common concerning the evaluation of the interventions was an overall grade participants assigned to the intervention. Although we can assume that this item gives an overall impression of participants' evaluation, it might be that participants with different educational levels liked and disliked different aspects of the intervention (e.g., layout, provided information, or personal relevance), which was not reflected in this overall grade. However, the evaluation of these different aspects was not equally assessed in all seven studies. Furthermore, it is possible that participants who did not like the intervention dropped out before completing the 
evaluation item. In this study, we included only those participants that completed the evaluation item and assessed follow-up at the subsequent measurement.

Second, all interventions were based on the I-Change model and targeted the same social cognitive determinants to change behavior, which allows for comparing the seven studies. However, a generalization of the results for other interventions must be done with caution because some interventions also used other theories, such as selfregulation theories, as a framework for the educational content.

Although the restricted number of studies is a limitation, including other Web-based tailored interventions might have resulted in even higher program heterogeneity and would have made comparisons even more difficult and results (partly) dependent on program characteristics. Also Wienert and Kuhlmann (Wienert \& Kuhlmann, 2015) have determined that tailored interventions are difficult to compare, whereas the interventions included in this study were comparable because they all were based on the same theoretical background (the l-Change model), all seven studies provided tailored feedback on social cognitive determinants from this model and provided feedback, and all seven studies included the same program evaluation item. The comparison of interventions using other tailoring techniques than the interventions described in this study is difficult and access to the original data at the individual level would be necessary for further research and adequate analysis (Noguchi, Albarracín, Durantini, \& Glasman, 2007).

One of the strengths of this study is the access to seven datasets (at participant level), which allowed us to conduct the analysis with the original data. Second, all studies used at least one identical item to assess the study evaluation which enables us to compare these studies. Finally, all studies had a large number of participants ranging from 1149 to 5055, which makes our results meaningful.

\section{CONCLUSION}

This study showed that for three of seven studies on computer-tailored interventions, participants with a lower educational level dropped out more often from follow-up measurements and tended to evaluate the interventions better compared to participants with a middle and higher educational level. However, the evaluation of the intervention did not predict participation or nonparticipation at follow-up. Based on our results, it is hard to say what other factors may play a role in dropout attrition from Web-based computer-tailored interventions. Further studies might evaluate different aspects of the intervention, besides only the participants' grade, to find more relevant aspects of intervention evaluation.

Future studies should take high dropout among lower educated participants into consideration when developing strategies to decrease high dropout from Web-based computer-tailored interventions. 


\section{ACKNOWLEDGMENTS}

We would like to thank all doctoral advisors who supported the development of the mentioned Web-based computer-tailored interventions: Catherine Bolman, Stef Kremers, Lilian Lechner, Anke Oenema, Liesbeth van Osch, Jean Muris, and Trudy van der Weijden.

\section{Authors' Contributions}

All authors contributed to this paper. DR conducted the analyses and drafted the paper. $\mathrm{HdV}$ and RC contributed to the design of the paper. All authors contributed to the interpretation of the data and to the writing of the paper. All authors revised the manuscript critically for important intellectual content and read and approved the final manuscript.

\section{Conflicts of Interests}

$\mathrm{HdV}$ is the scientific director of Vision2Health, a company that licenses evidence-based innovative computer-tailored health communication tools. The other authors declare that they have no competing interests. 


\section{Chapter 7}

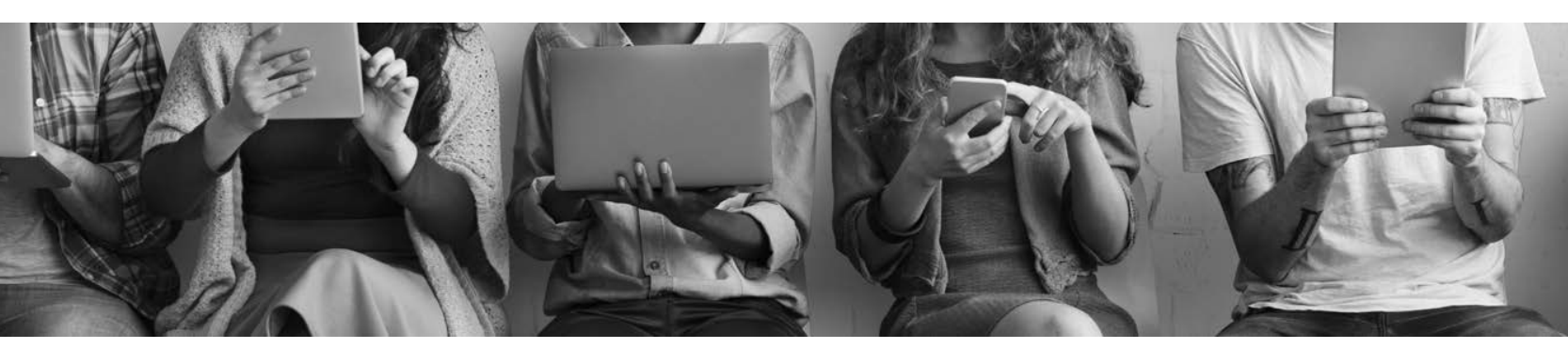

\section{General discussion}





\section{DISCUSSION}

The main aim of this dissertation is to describe the characteristics of the online behavior of people with different personal characteristics (i.e., gender, age, educational level, level of income, employment, and relationships). In the first part of this dissertation general online behavior differences were examined. Secondly, users' behavior on an educational website and potential differences between people with different personal characteristics were analyzed. Thirdly, behavior within Web-based computer tailored health interventions was assessed (i.e., number of intervention modules participated in and generation of action plans). Finally, study attrition from seven Web-based computer tailored health interventions were evaluated for people with different levels of education.

This chapter summarizes the most important findings and will elaborate on possible explanations for the differences in online behavior. Practical as well as methodological considerations and recommendations for further research will be discussed.

\section{Main findings}

With regards to the first aim, of determining whether Internet use differs among people with different personal characteristics, we found differences in seven explored Internet behaviors with regards to sociodemographic characteristics (i.e., age, gender, education, income, and employment). Activities regarding social interaction (emailing, social networking websites) were more popular among higher educated and employed people. Activities for personal development (reading news and information seeking) were clearly more performed by higher educated, higher income, and employed people, contrary to entertainment activities (gaming and watching movies) which were more popular among people with a lower educational level, the unemployed, and those with a lower level of income. Differences in Internet use might give reason to assume that individuals with different personal characteristics might also use webpages differently.

The second aim of this dissertation was to examine whether individuals with different personal characteristics varied in their usage of a website in terms of the level of visiting of sub-websites and additional content. Younger visitors and those with a higher educational level read more information on the evaluated website. Again, evidence was found showing that disadvantaged persons (lower educated) made less use of the evaluated website, which indicates that they also gained less information from it, and it could be assumed that the information presented on the website had little to no impact for them because being exposed to information and paying attention to it are the fundamental first steps in the process of gaining knowledge, and changing attitudes and behaviors (McGuire, 1989).

Regarding the third aim, we examined whether people with different personal characteristics made different use of Web-based health interventions (i.e., 
recommended amount of module participation and generating action plans). While Web-based interventions have been demonstrated to be effective, our results have shown that older participants, females, the unemployed, ill people, and those within a relationship followed the intervention recommendations more often. Following intervention recommendations, such as making action and coping plans, might increase behavior change, since a dose response relationship has been found between health behavior change and intervention participation (Donkin et al., 2013; Vroege et al., 2014). Concerning education and income, no differences were found within this study with regards to following the recommended intervention modules. In contrast to the previous studies from this dissertation (see Chapters 2, 3, and 4), in which clear differences were found regarding online use and several personal characteristics, the generation of action plans within a Web-based health intervention hardly differs for people with different personal characteristics.

With regards to the fourth aim, we explored dropout attrition among people with different educational levels via seven Web-based computer tailored health interventions (Chapter 6), as one of the main problems in Web-based interventions is the large dropout rate (Eysenbach, 2005). The results of our analyses showed that dropout rates were higher among lower and middle educated participants within three of the studies, in the other four studies, the difference did not reach significance. Dropout from follow-up measurements could be seen as an indication that participants did not use the intervention as recommended. This remains a problematic issue, especially for researchers, as intervention effects are difficult to prove in cases of high dropout attrition. Furthermore, we thought that a negative evaluation of these interventions could cause the dropout of lower educated participants, but this was not confirmed in the examined studies. Due to the fact that all studies were pretested on people with different levels of education, it could be assumed that the intervention was tailored to specific needs (e.g., layout and difficulty of information) and that the reasons for the high dropout rate must been sought elsewhere. As described earlier, not only it is important to reach people with a lower educational level (and others who are at risk) with health behavior change interventions, and so help them to improve their lifestyles but also important for researchers that people stay within the intervention in order to be able to collect information from follow-up measurements regarding the effectiveness of the intervention (Cunningham, 2014).

To summarize, the findings from this dissertation show that differences exists in online usage between people with different personal characteristics. These differences were found for seven Internet activities and the amount of time spent online, and differences exist regarding the amount of information people search on a health-related website, the amount of modules people accomplish within a Web-based computer tailored health intervention, and regarding dropout attrition form Web-based health interventions (see Table 7.1). 
Table 7.1: Overview of main study results presented in this dissertation

\begin{tabular}{|c|c|c|c|}
\hline Chapter & Research question & Study subject & Main outcomes \\
\hline Two & $\begin{array}{l}\text { 1. To what extent does } \\
\text { Internet usage differ } \\
\text { among people with } \\
\text { different personal } \\
\text { characteristics, and if so, } \\
\text { will the gap be closed } \\
\text { among those subgroups? }\end{array}$ & $\begin{array}{l}\text { Trend analysis of Internet } \\
\text { usage between 2009-2014 } \\
\text { among people with } \\
\text { different } \\
\text { sociodemographic } \\
\text { characteristics }\end{array}$ & $\begin{array}{l}\text { All Internet activities increased in use } \\
\text { besides being active in forums/blogs which } \\
\text { decreased. Information seeking is and was } \\
\text { the most popular online activity. Activities } \\
\text { regarding social interaction (mailing, social } \\
\text { network websites), entertainment (gaming, } \\
\text { movies), and activities for personal } \\
\text { development (reading news) increased. } \\
\text { Several differences within subgroups (age, } \\
\text { gender, education, income, occupation) } \\
\text { exists. }\end{array}$ \\
\hline Three & $\begin{array}{l}\text { 2. To what extent are } \\
\text { there differences in health- } \\
\text { related website usage } \\
\text { among people with } \\
\text { different personal } \\
\text { characteristics? }\end{array}$ & $\begin{array}{l}\text { Effects of visiting an } \\
\text { educational website about } \\
\text { tobacco additives }\end{array}$ & $\begin{array}{l}\text { Website visit was related to younger age, } \\
\text { higher education, and having a lower risk } \\
\text { perception towards tobacco additives. }\end{array}$ \\
\hline Four & $\begin{array}{l}\text { 3. To what extent are } \\
\text { personal characteristics } \\
\text { associated with Web- } \\
\text { based computer-tailored } \\
\text { health intervention usage } \\
\text { as recommended by } \\
\text { researchers? }\end{array}$ & $\begin{array}{l}\text { Web-based computer- } \\
\text { tailored intervention } \\
\text { aiming to improve } 5 \\
\text { different health lifestyle } \\
\text { behaviors }\end{array}$ & $\begin{array}{l}\text { Following the intervention recommendation } \\
\text { increased when participants were older, } \\
\text { female, unemployed, ill, or in a relationship. } \\
\text { No significant relevant differences were } \\
\text { found between groups with different levels } \\
\text { of education, incomes, or quality of life } \\
\text { regarding intervention use as recommended. }\end{array}$ \\
\hline Five & & $\begin{array}{l}\text { Web-based computer- } \\
\text { tailored planning } \\
\text { intervention aimed at } \\
\text { generating action and } \\
\text { coping plans to improve } \\
\text { fruit and vegetable } \\
\text { consumption and physical } \\
\text { activity. }\end{array}$ & $\begin{array}{l}\text { Concerning personal characteristics (age, } \\
\text { gender, education, relationship, occupation) } \\
\text { no differences were found for the generation } \\
\text { of action plans for physical activity and fruit } \\
\text { and vegetable consumption. The action plan } \\
\text { quality in terms of specificity is associated } \\
\text { with having a relationship, no differences for } \\
\text { action plan instrumentality were assessed } \\
\text { concerning different personal characteristics. }\end{array}$ \\
\hline Six & $\begin{array}{l}\text { 4. What role does } \\
\text { education level play in } \\
\text { dropouts from Web-based } \\
\text { computer-tailored health } \\
\text { interventions? }\end{array}$ & $\begin{array}{l}\text { Comparing dropout } \\
\text { attrition for people with } \\
\text { different educational level } \\
\text { from seven different Web- } \\
\text { based computer-tailored } \\
\text { interventions }\end{array}$ & $\begin{array}{l}\text { In three of seven studies we found a higher } \\
\text { study dropout attrition rate among } \\
\text { participants with a lower educational level. } \\
\text { In two of these studies middle educated } \\
\text { participants had a higher dropout attrition } \\
\text { rate compared to highly educated } \\
\text { participants. In four studies no significant } \\
\text { difference was found among people with } \\
\text { different levels of education concerning } \\
\text { dropout attrition. }\end{array}$ \\
\hline
\end{tabular}




\section{Explanations for online inequalities}

The results presented in this dissertation indicate that equality in Internet access does not ensure equality in Internet usage. People use the Internet and Web-based computer tailored health interventions differently and disadvantaged people more often use them in an undesirable way. Based on the human principal of "equality of opportunities" (Arneson, 2015), it is a significant public health concern to find possible reasons for these inequalities in order to be able to provide Web-based information and interventions that can be used equally by people with different personal characteristics. Next, in order to access to information and interventions, adequate use is also imperative so as to achieve health behavior change, since studies have demonstrated a dose-response relationship between participation and behavior change (Donkin et al., 2013; Elfeddali, Bolman, Candel, et al., 2012; Vroege et al., 2014). The following section will discuss theoretical and empirical explanations for the observed differences in online use, and these thoughts will be linked to the findings from this dissertation.

\section{Theory of Acceptance and Use of Technology and Uses and Gratification Theory}

Based on the comparison of eight different psychological theories and models the Unified Theory of Acceptance and Use of Technology (UTAUT) has been developed in order to explain and describe the acceptance and use of IT (Venkatesh, Morris, Davis, \& Fred, 2003). The UTAUT assumes that the intention to make use of IT (such as Webbased information/interventions) is based on four determinants: performance expectancy (perceived usefulness), effort expectancy (perceived ease of use), social influence (perceived social norm of use), and facilitating conditions (perceived support of use). Mediating factors are age, gender, level of experience, and voluntariness. According to the UTAUT, it can be assumed that the higher the perceived usefulness and ease of use, the higher the intention for IT usage. These extrinsic motivations have been evaluated in different studies (Li, 2010; Luo \& Remus, 2014). Furthermore, intrinsic motivations such as enjoyment have also been found to be related to technology use. These motivations can be explained by the Uses and Gratification (U\&G) theory (Ruggiero, 2000), which assumes that people make actively use of media (such as the Internet) with the aim of satisfying specific needs and goals, which could vary from seeking information, being entertained, increasing social interaction, or escaping from day-to-day stress (McQuail, 2010). The U\&G theory helps to explain the motivation for Internet use from customer perspective by asking what brings people to the Internet and what makes them stay (Stafford, Stafford, \& Schkade, 2004). As we have seen that people with different personal characteristics make different use of the Internet, health related websites, and Web-based computer-tailored interventions, it could be expected that using Web-based information is estimated differently in terms of ease of use and usefulness, and that it provides different people with different gratifications. 
For example, the results from Chapter 2 of this dissertation have shown that higher educated people's use of the Internet is more often information focused (i.e., searching for information and reading news), while people with a lower educational level's use of the Internet is more entertainment orientated (playing online games and watching movies), which is also in line with other studies (e.g., van Deursen \& Van Dijk, 2015; van Deursen et al., 2015). As websites that provide health related information have the purpose of informing the public in a neutral and objective manner, it can be assumed that these websites are attractive to those people who are looking for information, but are less appealing for people who also receive gratification from entertainment. Increasing the entertainment factor within health related websites and Web-based computer tailored health interventions could be one possible strategy for increasing intervention use and participation in follow-up measurements. Based on the SelfDetermination Theory (Ryan \& Deci, 2000), entertainment is related to the perceived level of autonomy of one's own actions, competence; (which refers to the balance between one's own skills and ability to solve a task), and relatedness (which refers to personal relationships). In order to increase entertainment, it should be taken into consideration that the tasks that participants have to fulfil should not exceed their skills (e.g., attention span after filling in a long questionnaire), thereby giving participants the feeling that they have autonomy within the intervention (e.g., by choosing the amount of information they would like to receive or the number of modules they would like to participate in within a session) (Crutzen, van 't Riet, \& Short, 2016). Increasing interactivity has also shown positive results with regards to intervention use and engagement (e.g., Pang, Chang, Verspoor, \& Pearce, 2016; Poirier \& Cobb, 2012). Moreover, a few studies have reported on the use of entertainment elements within their interventions. Combining non-health related content, for example within an email as a reminder to participate within an intervention or on the intervention website, such as short local news, weather forecasts, or other information that is of interest to people and entertaining them without being too strongly related to the actual intervention (Klasnja \& Pratt, 2012). With the use of such broad information, the entertainment factor could be addressed and interest could be maintained. People using the Internet in order to search for health information or to participate in a Web-based health intervention might often not achieve gratification because of their bad condition and may consequently avoid these sources in the future, since people avoid selfstigmatization (Nam \& Lee, 2015; Teh, King, Watson, \& Liu, 2014).

These differences in gratification might explain the differences in online use as presented in this dissertation. Future research might focus on strategies aiming to make online use more attractive to people with different personal characteristics in order to reduce online usage inequalities. 


\section{Health Literacy}

In order to be able to use information, people need the ability to search, understand, evaluate, and apply the appropriate information. In the Netherlands, $11 \%$ of the general population have low levels of literacy (Organisation for Economic Co-operation and Development, 2000). The percentage with lower levels of health literacy (which refers to the ability to use health related information) is even lower in the Netherlands at $29 \%$ (World Health Organization, 2013). EHealth literacy expands health literacy to the ability to make use of information that is provided electronically, such as from websites or Web-based health interventions. It can be argued that the current level of eHealth literacy may even be below the level of health literacy, because handling electronic devices and Web-based information is even more demanding. These thoughts are worrisome since the Internet has become a popular medium for spreading (health) related information, and it could be assumed that people with low levels of eHealth literacy are not able to benefit from this information because of inequalities in use. Those with higher levels of eHealth literacy were found to be younger, male, higher educated, to have a higher income, and to make more use of Web-based information (Chesser, Burke, Reyes, \& Rohrberg, 2016; Choi \& DiNitto, 2013; Neter \& Brainin, 2012; Stellefson et al., 2011). The results from this dissertation (see Chapters 2, 3, 4, and 6) revealed that people with different personal characteristics make different use of Webbased information and interventions, which is in line with previous studies. Therefore, it could be assumed that people who did not use the Web-based information and interventions as recommended had lower levels of eHealth literacy. People with lower levels of eHealth literacy need to use more effort to understand information and find information on websites, they need more time within an intervention, and they might therefore use these interventions less, because of a higher threshold of cognitive exhaustion. Studies have revealed that people with lower levels of health literacy make less use of preventative service and have less health related knowledge, poorer levels of health, and even increased mortality (Andrus \& Roth, 2002; Baker et al., 2007; P. J. Schulz \& Nakamoto, 2013).

EHealth literacy requires different skills such as traditional literacy, health literacy, information literacy, scientific literacy, media literacy, and computer literacy (C. D. Norman \& Skinner, 2006). In order to improve website and Web-based intervention use, it might be helpful to include these skills in educational curricula for students. Additionally, people who have left the school system could be supported with interventions to improve their eHealth literacy. Studies have shown promising results in terms of increasing eHealth literacy (Watkins \& Xie, 2014). The review of Watkins and Xie identified four interventions (out of 23 identified studies) that made use of tailoring techniques. This has also been proven to be effective for increasing participants' eHealth literacy. These interventions made use of instructions on how to use a specific website. Nevertheless, these interventions are scarce, and it is recommended that we 
should develop more interventions targeting eHealth literacy. Furthermore, existing (and effective) Web-based health interventions may need to be adjusted and tailored to the level of eHealth literacy of participants in order to guide them through a specific intervention (e.g., by providing instructions on how to use the intervention, explaining navigation, or tailoring the amount of information that participants can process). Adjusting Web-based information on websites and improving Web-based interventions might increase use among those people who have found using these applications to be less beneficial (e.g., those with lower levels of education or older people; see Chapters 2, 3, 4, and 6). Improving online use would be beneficial because the identified differences in patterns of online use provide reason to assume that people who are most at risk of poor health behaviors will continue to be disadvantaged due to a lack of information and behavior change support via the Internet.

\section{Health inequalities and personal characteristics}

In addition to proximal mechanisms that could influence differences in Web-based health related usage, such as gratification of use or skills like eHealth literacy, other more indirect or distal factors could be associated with differences in online use as well. From studies regarding health inequality and socioeconomic differences, it is known that perceived lack of control of one's own life can cause differences in health (Lachman, Neupert, \& Agrigoroaei, 2011; Lachman \& Weaver, 1998; T. C. Murray, Rodgers, \& Fraser, 2012). People who perceive a lack of control in their work environment and in their daily lives have higher levels of stress and more often have health conditions such as depression and other mental and psychological problems (Koster et al., 2006). People with lower levels of income, disadvantaged occupational situations, and those who are unemployed report the lowest levels of perceived control (Poortinga, Dunstan, \& Fone, 2008). It is known that people with a lower socioeconomic status have lower levels of perceived control, and these people might have learned that they have less control over their lives and that they have limited abilities to influence their health situations (Bailis, Segall, Mahon, Chipperfield, \& Dunn, 2001; Bauer, Bittlingmayer, \& Richter, 2008; Bobak, Pikhart, Rose, Hertzman, \& Marmot, 2000; Poortinga et al., 2008). Furthermore, it is also known that there are differences in time perspectives among people with different socioeconomic characteristics. Studies revealed that people with a future time perspective are more engaged in preventative behaviors such as healthy eating habits or physical activity (Adams, 2009; Griva, Tseferidi, \& Anagnostopoulos, 2015; Mahon, Yarcheski, \& Yarcheski, 1997). Taking these considerations into account, it is possible that people with disadvantaged personal characteristics do not invest in their health because they are not convinced that they have the ability to control it and so might not perceive the long-term results (Guthrie, Butler, Lessl, Ochi, \& Ward, 2014; Ward, Guthrie, \& Butler, 2009). While self-efficacy is a direct predictor of health behavior, studies have shown that control beliefs moderate this pathway (Wallston, 1992). The Web-based 
health interventions presented in this dissertation were tailored for increasing selfefficacy with regards to performing specific health behaviors but it could also be useful to focus on control beliefs, especially in people with disadvantaged personal characteristics. Furthermore, long-term results should be emphasized within health interventions in order to increase the perceived importance of behavior change for people with lower levels of future perspectives. These strategies might also improve intervention use among people with different personal characteristics and, consequently, increases the impact of health behavior interventions.

In summary, the studies within this dissertation have found evidence of inequalities in Internet use among people with different personal characteristics. While the presented studies did not focus on the reasons for these differences, this chapter has discussed possible explanations. Further research might aim to assess the mediating role of usefulness, gratification, health literacy, control beliefs, and time perspectives in order to improve Internet use among people with disadvantaged personal characteristics.

\section{RECOMMENDATIONS FOR FURTHER RESEARCH}

The present dissertation assessed personal characteristics as independent variables of online and Web-based computer tailored intervention use. Besides the studies described in Chapters 4 and 5, we made use of secondary data analysis, and Chapter 6 made use of seven different studies in which education was the only matching denominator. Due to the given studies, we were restricted to the given variables. These personal characteristic variables should not be equated with user characteristics such as information processing abilities, interest, or eHealth literacy skills. Those variables were not assessed within these studies, but could be related to online and intervention use (e.g., Chesser et al., 2016; Diviani et al., 2015). For example, there is a positive relationship between educational level and the ability to process and understand health related information (Diviani et al., 2015). Personal characteristics, such as level of education or occupation, could be seen as proxies for other determinants, such as cognitive processing abilities, which in turn could have an influence on interest, the time people spend online, or the amount of information someone will read, understand, and follow appropriately. There are ambiguous findings regarding socioeconomic status and cognitive function; some studies have found that people with lower socioeconomic status in childhood have poorer cognitive functions later in life, but other studies did not find such a relationship (A. D. Murray et al., 2012; Staff, Chapko, Hogan, \& Whalley, 2016). Further studies might aim to assess users' characteristics, such as information processing abilities, in addition to personal characteristics in order to determine whether these variables influence online and Web-based health intervention use, enabling the identification of strategies that could be used in order to increase online usage among people with different personal characteristics. 
Since studies reveal that satisfaction with media (i.e., websites and interventions) can be an important factor for use (Luo \& Remus, 2014; Ruggiero, 2000), we should consider including items about satisfaction within intervention evaluations. The studies included in this dissertation (particularity in Chapter 6, which demonstrated that intervention evaluation did not predict dropout attrition) assessed intervention satisfaction by including items about the understandability of the given recommendations, the layout, whether participants had the intention to revisit the intervention, whether they thought the intervention was useful, etc., but aspects such as pleasure, fun, and entertainment were not assessed. These variables should be included in future website and Web-based intervention evaluations in order to investigate which aspects of the intervention are evaluated positively. Further studies are necessary in order to determine which aspects of Web-based interventions provide pleasure and satisfaction.

This dissertation provides evidence of differences in online use between people with different personal characteristics (e.g., lower educated people participated in fewer intervention modules and had higher rates of dropout attrition), but only a few studies have explored intervention use within their datasets. It would be beneficial if this topic received more attention within the field of usage of Web-based health intervention. While dropout rates form Web-based interventions are considered in most interventional studies (e.g., Christensen \& Mackinnon, 2006; Crutzen, Viechtbauer, Spigt, \& Kotz, 2014; Geraghty et al., 2010; Moroshko et al., 2011; Neve et al., 2010; Price et al., 2012), intervention usage is less often investigated. Data and information regarding the starting of the intervention, module completion, and interacting with the intervention can be easily obtained from the server. It would be beneficial if researchers also paying attention to intervention usage and personal characteristics in order to confirm (or disprove) the findings of this dissertation. More certainty would help us to find usage patterns of people with different personal characteristics and might enable researchers to tailor Web-based health related information and interventions to these personal characteristics.

Experimental studies are necessary to find causes and solutions for differences in online and intervention use among people with different personal characteristics. One possibility may be to develop a Web-based computer tailored intervention (or make use of an already validated Web-based intervention) and include tailoring strategies to personal characteristics. For example, it is possible that people with a lower educational level and lower levels of income have less interest in changing their health behavior, because they do not perceive the long-term benefits. Health behavior change interventions might need to stress their aims differently for different target populations. Furthermore, for people who already behave unhealthily, recommendations with guidelines might be too demanding, certainly if the discrepancy between their actual and desired behavior is high. These participants may then opt to avoid participation or drop out early from interventions. Research is needed in order to assess whether this 
occurs. If so, an alternative strategy to be tested could be to let participants decide how much they think they can change (e.g., increasing physical activity by a small amount each week instead of wanting people to be active 30 minutes each day). Smaller changes could increase participants' self-efficacy, their control beliefs, and their enjoyment of the intervention if small successes can be achieved.

\section{GENERAL CONCLUSION}

The studies presented in this dissertation showed that people with different personal characteristics made different use of the Internet, health related websites, and Webbased computer tailored health interventions. For example, people with a lower educational level or lower income used the Internet more for entertainment purposes than for personal enhancement. These results further demonstrated that people with a lower education level visited a health related website less intensively than higher educated people. Furthermore, differences with regard to the recommendation of module use within a Web-based health intervention also differed between people with different personal characteristics. Interestingly, intervention use regarding the generation of action plans did not reveal differences between people with different educational levels, incomes, occupations, or ages. Finally, dropout attrition was found to be associated with lower levels of education. Surprisingly, participants with a lower educational level evaluated the intervention more positively, which may be partly explained by the fact that most of the interventions used panels that also represented lower educated respondents who could indicate preferences and comment on draft versions of the interventions. Intervention usage might be improved if participants receive clear instructions on how to use the intervention (see, for example, Chapter 6; generating action plans). Nevertheless, the inequalities of online usage is worrisome because disadvantaged people made less beneficial use of these sources, which could increase health inequalities among those people.

While this dissertation demonstrated that inequalities in online use exist between people with different personal characteristics, further research is necessary in order to gain more insight into the underlying mechanisms of this online behavior. Recommendations regarding changing websites and Web-based interventions have been given but experimental studies are necessary in order to determine which strategies improve online usage. 
Adams, J. (2009). The mediating role of time perspective in socio-economic inequalities in smoking and physical activity in older English adults. Journal of Health Psychology, 14(6), 794-799.

Adler, N. E., \& Ostrove, J. M. (1999). Socioeconomic status and health: What we know and what we don't. Annals of the New York Academy of Sciences, 896(1), 3-15.

Adriaanse, M. A., Vinkers, C. D. W., De Ridder, D. T. D., Hox, J. J., \& De Wit, J. B. F. (2011). Do implementation intentions help to eat a healthy diet? A systematic review and meta-analysis of the empirical evidence. Appetite, 56(1), 183-193.

Aerschot, L. V., \& Rodousakis, N. (2008). The link between socio-economic background and Internet use: barriers faced by low socio-economic status groups and possible solutions. Innovation: The European Journal of Social Science Research, 21(4), 317-351.

Ajzen, I. (1991). The theory of planned behavior. Organizational Behavior and Human Decision Processes, 50, 179-211.

Akkermans, M. M. P., Cleassen, P. J. G. M., Donselaar, P., Heerschap, N. M., de Heij, H. N., Kuijpers, A. D., Nieweboer, J. M. M. J., \& Sleipen, G. P. E. A. (2012). ICT, kennis en economie CBS (Ed.), Retrieved from http://www.cbs.nl/NR/rdonlyres/130F8419-05C1-43AE-B5ED-4C373F34EC82/0/2012i78pub.pdf

American Psychological Association, \& Task Force on Socioeconomic Status. (2007). Report of the APA Task Force on socioeconomic status. Retrieved from https://www.apa.org/pi/ses/resources/publications/taskforce-2006.pdf

Ammann, R., Vandelanotte, C., de Vries, H., \& Mummery, W. K. (2013). Can a website-delivered computertailored physical activity intervention be acceptable, usable, and effective for older people? Health Education \& Behavior, 40(2), 160-170.

Andrus, M. R., \& Roth, M. T. (2002). Health literacy: A review. Pharmacotherapy: The Journal of Human Pharmacology and Drug Therapy, 22(3), 282-302.

Arneson, R. (2015). Equality of opportunity. The Stanford Encyclopedia of Philosophy. Retrieved from plato.stanford.edu/archives/sum2015/entries/equal-opportunity

Bailis, D. S., Segall, A., Mahon, M. J., Chipperfield, J. G., \& Dunn, E. M. (2001). Perceived control in relation to socioeconomic and behavioral resources for health. Social Science \& Medicine, 52(11), 1661-1676.

Baker, D. W., Wolf, M. S., Feinglass, J., Thompson, J. A., Gazmararian, J. A., \& Huang, J. (2007). Health literacy and mortality among elderly persons. Archives of Internal Medicine, 167(14), 1503-1509.

Bartholomew, L. K., Parcel, G. S., Kok, G., Gottlieb, N. H., \& Fernández, M. E. (2011). Planning health promotion programs: An Intervention Mapping approach. San Francisco: John Wiley \& Sons.

Bauer, U., Bittlingmayer, U. H., \& Richter, M. (2008). Health inequalities. Determinanten und Mechanismen gesundheitlicher Ungleichheit. Wiesbaden: VS Verlag für Sozialwissenschaften.

Baumeister, R. F., Heatherton, T. F., \& Tice, D. (1994). Losing control: How and why people fail at selfregulation. San Diego, CA: Academic Press.

Baumeister, R. F., Muraven, M., \& Tice, D. M. (2000). Ego depletion: A resource model of volition, selfregulation, and controlled processing. Social Cognition, 18(2), 130-150.

Belanger-Gravel, A., Godin, G., \& Amireault, S. (2013). A meta-analytic review of the effect of implementation intentions on physical activity. Health Psychology Review, 7(1), 23-54.

Bell, A. V. (2014). "I think about Oprah" social class differences in sources of health information. Qualitative Health Research, 24(4), 506-516.

Benjamini, Y., \& Hochberg, Y. (1995). Controlling the false discovery rate: A practical and powerful approach to multiple testing. Journal of the Royal Statistical Society. Series B (Methodological), 57(1), 289-300.

Benjamini, Y., \& Yekutieli, D. (2001). The control of the false discovery rate in multiple testing under dependency. The Annals of Statistics, 29(4), 1165-1188.

Bennett, G. G., \& Glasgow, R. E. (2009). The delivery of public health interventions via the Internet: Actualizing their potential. Annual Review of Public Health, 30(1), 273-292.

Bierings, H. (2013). Onderwijsniveau bevolking gestegen. Centraal Bureau voor de Statistiek. Retrieved from http://www.cbs.nl/nl-NL/menu/themas/onderwijs/publicaties/artikelen/archief/2013/2013-3905wm.htm 
Bleich, S. N., Jarlenski, M. P., Bell, C. N., \& LaVeist, T. A. (2012). Health inequalities: Trends, progress, and policy. Annual Review of Public Health, 4(33), 7-40.

Bobak, M., Pikhart, H., Rose, R., Hertzman, C., \& Marmot, M. (2000). Socioeconomic factors, material inequalities, and perceived control in self-rated health: Cross-sectional data from seven post-communist countries. Social Science \& Medicine, 51(9), 1343-1350.

Boering, H., Bechthold, A., Bub, A., Ellinger, S., Haller, D., Kroke, A., Leschik-Bonnet, E., Müller, M. J., Oberritter, H., Schulze, M., Stehle, P., \& Watzl, B. (2012). Stellungnahme, Gemüse und Obst in der Prävention ausgewäh/ter chronischer Krankheiten. Retrieved from http://kgm.pebonline.de/ uploads/tx_news/DGE-Stellungnahme-Gemuese-Obst-2012_02.pdf

Boneva, B., Kraut, R., \& Frohlich, D. (2001). Using E-mail for personal relationships: The difference gender makes. American Behavioral Scientist, 45(3), 530-549.

Bonevski, B., Randell, M., Paul, C., Chapman, K., Twyman, L., Bryant, J., Brozek, I., \& Hughes, C. (2014). Reaching the hard-to-reach: A systematic review of strategies for improving health and medical research with socially disadvantaged groups. BMC Medical Research Methodology, 14(1), 1-29.

Bonfadelli, H. (2002). The Internet and knowledge gaps: A theoretical and empirical investigation. European Journal of Communication, 17(1), 65-84.

Boon, B., Risselada, A., Huiberts, A., Riper, H., \& Smit, F. (2011). Curbing alcohol use in male adults through computer generated personalized advice: Randomized controlled trial. Journal of Medical Internet Research, 13(2), e43.

Borenstein, M., Hedges, L. V., Higgins, J. P. T., \& Rothstein, H. R. (2009). Introduction to meta-analysis (Vol. 1): John Wiley \& Sons.

Brandtweiner, R. (2010). How to become a sophisticated user: a two-dimensional approach to e-literacy. New media \& society, 12(5), 813-833.

Brashers, D. E., Goldsmith, D. J., \& Hsieh, E. (2002). Information seeking and avoiding in health contexts. Human Communication Research, 28(2), 258-271.

Brewer, N. T., Chapman, G. B., Gibbons, F. X., Gerrard, M., McCaul, K. D., \& Weinstein, N. D. (2007). Metaanalysis of the relationship between risk perception and health behavior: The example of vaccination. Health Psychology, 26(2), 136-145.

Brodie, M., Flournoy, R. E., Altman, D. E., Blendon, R. J., Benson, J. M., \& Rosenbaum, M. D. (2000). Health information, the Internet, and the digital divide. Health Affairs, 19(6), 255-265.

Broekhuizen, K., Kroeze, W., van Poppel, M. N. M., Oenema, A., \& Brug, J. (2012). A systematic review of randomized controlled trials on the effectiveness of computer-tailored physical activity and dietary behavior promotion programs: An update. Annals of Behavioral Medicine: A publication of the Society of Behavioral Medicine, 44(2), 259-286.

Brouwer, W., Kroeze, W., Crutzen, R., de Nooijer, J., de Vries, N. K., Brug, J., \& Oenema, A. (2011). Which intervention characteristics are related to more exposure to Internet-delivered healthy lifestyle promotion interventions? A systematic review. Journal of Medical Internet Research, 13(1), e2-41.

Brug, J., Campbell, M., \& van Assema, P. (1999). The application and impact of computer-generated personalized nutrition education: A review of the literature. Patient Education and Counseling, 36(2), 145-156.

Brug, J., Oenema, A., \& Campbell, M. (2003). Past, present, and future of computer-tailored nutrition education. The American Journal of Clinical Nutrition, 77(4), 1028-1034.

Brug, J., Steenhuis, I., van Assema, P., \& de Vries, H. (1996). The impact of a computer-tailored nutrition intervention. Preventive Medicine, 25(3), 236-242.

Bundorf, M. K., Baker, L., Singer, S., \& Wagner, T. (2004). Consumer demand for health information on the Internet. Retrieved from http://www.nber.org/papers/w10386

CBS. (2014a). Dutch internet skills above EU average. Retrieved from http://www.cbs.nl/enGB/menu/themas/vrije-tijd-cultuur/publicaties/artikelen/archief/2014/2014-4066-wm.htm

CBS. (2014b). ICT gebruik van personen naar persoonskenmerken. Centraal Bureau voor de Statistiek. Retrieved from http://statline.cbs.nl/StatWeb/publication/?VW=T\&DM=SLNL\&PA=71098ned\&LA=NL 
CBS. (2014c). Internetgebruik 65- tot 75-jarigen. Retrieved from http://www.cbs.nl/nl-NL/menu/themas/vrijetijd-cultuur/publicaties/artikelen/archief/2013/2013-4005-wm.htm

CCMO. (2015). Uw onderzoek: WMO-plichtig of niet. Retrieved from http://www.ccmo.nl/nl/uw-onderzoekwmo-plichtig-of-niet

Celik, V., \& Yesilyurt, E. (2013). Attitudes to technology, perceived computer self-efficacy and computer anxiety as predictors of computer supported education. Computers \& Education, 60(1), 148-158.

Charafeddine, R., Demarest, S., Van der Heyden, J., Tafforeau, J., \& Van Oyen, H. (2013). Using multiple measures of inequalities to study the time trends in social inequalities in smoking. The European Journal of Public Health, 23(4), 546-551.

Charness, N., \& Boot, W. R. (2009). Aging and information technology use: Potential and barriers. Current Directions in Psychological Science, 18(5), 253-258.

Chatzisarantis, N., Wood, C., Stiff, C., \& Hagger, M. (2010). Self-regulation and self-control in exercise: the strength-energy model. International Review of Sport and Exercise Psychology, 3(1), 62-86.

Chesser, A., Burke, A., Reyes, J., \& Rohrberg, T. (2016). Navigating the digital divide: A systematic review of eHealth literacy in underserved populations in the United States. Informatics for Health and Social Care, 41(1), 1-19.

Ching, C., Basham, J., \& Jang, E. (2005). The legacy of the digital divide. Urban Education, 40(4), 394.

Choi, N. G., \& DiNitto, D. M. (2013). The digital divide among low-income homebound older adults: Internet use patterns, eHealth literacy, and attitudes toward computer/Internet use. Journal of Medical Internet Research, 15(5), e93.

Christensen, H., Griffiths, K. M., \& Farrer, L. (2009). Adherence in Internet interventions for anxiety and depression: Systematic review. Journal of Medical Internet Research, 11(2), e13.

Christensen, H., \& Mackinnon, A. (2006). The law of attrition revisited. Journal of Medical Internet Research, 8(3), e20.

Cline, R. J. W., \& Haynes, K. M. (2001). Consumer health information seeking on the Internet: The state of the art. Health Education Research, 16(6), 671-692.

Committee on Health and Behavior: Research practice and policy board on neuroscience and behavioral health. (2001). Health and behavior: The interplay of biological, behavioral, and societal influences. Washington (DC): National Academies Press (US).

Compaine, B. M. (2001). The digital divide: Facing a crisis or creating a myth? Cambridge, MA [etc.]: The MIT Press.

Compernolle, S., Vandelanotte, C., Cardon, G., De Bourdeaudhuij, I., \& De Cocker, K. (2015). Effectiveness of a web-based, computer-tailored, pedometer-based physical activity intervention for adults: A cluster randomized controlled trial. Journal of Medical Internet Research, 17(2), e38.

Cooper, J. (2006). The digital divide: The special case of gender. Journal of Computer Assisted Learning, 22(5), 320-334.

Costa-Font, J., Mossialos, E., \& Rudisill, C. (2009). When is the Internet a valued communication device for health information in Europe? Economics of Innovation and New Technology, 18(5), 429-445.

Cotten, S. R., \& Gupta, S. S. (2004). Characteristics of online and offline health information seekers and factors that discriminate between them. Social Science \& Medicine, 59(9), 1795-1806.

Cowell, A. J. (2006). The relationship between education and health behavior: Some empirical evidence. Health Economics, 15(2), 125-146.

Craig, C. L., Marshall, A. L., Sjostrom, M., Bauman, A. E., Booth, M. L., Ainsworth, B. E., Pratt, M., Ekelund, U., Yngve, A., Sallis, J. F., \& Oja, P. (2003). International physical activity questionnaire: 12-country reliability and validity. Medicine \& Science in Sports Exercise, 35, 1381-1395.

Crutzen, R., Beekers, N., van Eenbergen, M., Becker, M., Jongen, L., \& van Osch, L. (2014). E-loyalty towards a cancer information website: Applying a theoretical framework. Psycho-Oncology, 23(6), 685-691.

Crutzen, R., Cyr, D., \& de Vries, N. K. (2011). Bringing loyalty to e-Health: Theory validation using three Internet-delivered interventions. Journal of Medical Internet Research, 13(3), e73. 
Crutzen, R., Cyr, D., \& de Vries, N. K. (2012). The role of user control in adherence to and knowledge gained from a website: randomized comparison between a tunneled version and a freedom-of-choice version. Journal of Medical Internet Research, 14(2).

Crutzen, R., de Nooijer, J., Brouwer, W., Oenema, A., Brug, J., \& de Vries, N. K. (2011). Strategies to facilitate exposure to Internet-delivered health behavior change interventions aimed at adolescents or young adults: A systematic review. Health Education \& Behavior, 38(1), 49-62.

Crutzen, R., \& Ruiter, R. (2015). Interest in behavior change interventions: A conceptual model. The European Health Psychologist, 17(1), 6-11.

Crutzen, R., van 't Riet, J., \& Short, C. E. (2016). Enjoyment: A conceptual exploration and overview of experimental evidence in the context of games for health. Games for Health Journal, 5(1), 15-20.

Crutzen, R., Viechtbauer, W., Spigt, M., \& Kotz, D. (2014). Differential attrition in health behaviour change trials: A systematic review and meta-analysis. Psychology \& Health, 122-134.

Csikszentmihalyi, M. (1997). Finding flow: The psychology of engagement with everyday life: Basic Books.

Cuijpers, P., van Straten, A., \& Andersson, G. (2008). Internet-administered cognitive behavior therapy for health problems: A systematic review. Journal of Behavioral Medicine, 31(2), 169-177.

Cumming, G. (2011). Understanding the new statistics: Effect sizes, confidence intervals, and meta-analysis: Routledge.

Cummings, K. M., Hyland, A., Giovino, G. A., Hastrup, J. L., Bauer, J. E., \& Bansal, M. A. (2004). Are smokers adequately informed about the health risks of smoking and medicinal nicotine? Nicotine \& Tobacco Research, 6(3), 333-340.

Cunningham, A. J. (2014). Intervention adherence is related to participant retention: Implications for research. Journal of Medical Internet Research, 16(5), e133.

Curioni, C., \& Lourenco, P. (2005). Long-term weight loss after diet and exercise: A systematic review. International Journal of Obesity, 29(10), 1168-1174.

Cutler, D. M., \& Lleras-Muney, A. (2010). Understanding differences in health behaviors by education. Journal of Health Economics, 29(1), 1-28.

Dahlgren, D., \& Whitehead, M. (2007). European strategies for tackling social inequalities in health: Levelling up part 2. Retrieved from http://www.thehealthwell.info/node/91930

De Irala-Estevez, J., Groth, M., Johansson, L., Oltersdorf, U., Prattala, R., \& Martinez-Gonzalez, M. (2000). A systematic review of socio-economic differences in food habits in Europe: consumption of fruit and vegetables. European Journal of Clinical Nutrition, 54(9), 706-714.

de Jong de Josselin, S., Candel, M. J. J. M., Segaar, D., Cremers, H.-P., \& de Vries, H. (2014). Efficacy of a webbased computer-tailored smoking prevention intervention for Dutch adolescents: Randomized controlled trial. Journal of Medical Internet Research, 16(3).

De Leon, E., Fuentes, L., \& Cohen, J. E. (2014). Characterizing periodic messaging interventions across health behaviors and media: Systematic review. Journal of Medical Internet Research, 16(3), e93.

de Vet, E., Oenema, A., \& Brug, J. (2011). More or better: Do the number and specificity of implementation intentions matter in increasing physical activity? Psychology of Sport and Exercise, 12(4), 471-477.

de Voss, K. (2011). Imputation of income in household questionnaire LISS panel. Retrieved from http://www.lissdata.nl/dataarchive/study_units/view/323

de Vries, H., \& Brug, J. (1999). Computer-tailored interventions motivating people to adopt health promoting behaviors: introduction to a new approach. Patient Education and Counseling, 36, 99-195.

de Vries, H., Eggers, S., \& Bolman, C. (2013). The role of action planning and plan enactment for smoking cessation. BMC Public Health, 13(1), 393.

de Vries, H., Mesters, I., van der Steeg, H., \& Honing, C. (2005). The general public's information needs and perceptions regarding hereditary cancer: An application of the Integrated Change Model. Patient Education and Counseling, 56(2), 154-165.

de Vries, H., Mudde, A., Leijs, I., Charlton, Vartiainen, E., Buijs, G., Clemente, M. P., Storm, H., Navarro, A. G., Nebot, M., Prins, T., \& Kremers, S. (2003). The European Smoking prevention Framework Approach (EFSA): An example of integral prevention. Health Education Research, 18(5), 611-626. 
de Vries, H., van Osch, L., Eijmael, K., Smerecnik, C., \& Candel, M. (2012). The role of risk perception in explaining parental sunscreen use. Psychology \& Health, 1-17.

Debrand, C. C., \& Johnson, J. J. (2008). Gender differences in email and instant messaging: A study of undergraduate business information systems students. Journal of Computer Information Systems, 48(3), 20-30.

Deci, E. L., \& Ryan, R. M. (2011). Self-determination theory. In Lange, P., A., M., Kruglanski, A. W., \& Higgins, E. T. (Eds.), Handbook of theories of social psychology, 1, 416-433. Sage.

DiMaggio, P., \& Hargittai, E. (2001). From the 'digital divide 'to 'digital inequality': Studying Internet use as penetration increases. Princeton: Center for Arts and Cultural Policy Studies, Woodrow Wilson School, Princeton University, 4(1), 4-2.

Diviani, N., van den Putte, B., Giani, S., \& van Weert, C. M. J. (2015). Low health literacy and evaluation of online health information: A systematic review of the literature. Journal of Medical Internet Research, 17(5), e112.

Donkin, L., Christensen, H., Naismith, S. L., Neal, B., Hickie, I. B., \& Glozier, N. (2011). A systematic review of the impact of adherence on the effectiveness of e-therapies. Journal of Medical Internet Research, 13(3), e52.

Donkin, L., Hickie, I. B., Christensen, H., Naismith, S. L., Neal, B., Cockayne, N. L., \& Glozier, N. (2013). Rethinking the dose-response relationship between usage and outcome in an online intervention for depression: Randomized controlled trial. Journal of Medical Internet Research, 15(10), e231.

Drost, R. M. W. A., Paulus, A. T. G., Jander, A. F., Mercken, L., de Vries, H., Ruwaard, D., \& Evers, S. M. A. A. (2016). A web-based computer-tailored alcohol prevention program for adolescents: Cost-effectiveness and benefits. Journal of Medical Internet Research, 18(4), e93.

Dunn, T. J., Baguley, T., \& Brunsden, V. (2014). From alpha to omega: A practical solution to the pervasive problem of internal consistency estimation. British Journal of Psychology, 105(3), 399-412.

Dutton, W. H., \& Blank, G. (2011). Next generation users: The Internet in Britain. Oxford Internet Survey Report. Retrieved from http://www.worldinternetproject.net/_files/_Published/23/820_oxis2011_report.pdf

Eastin, M. S., \& LaRose, R. (2000). Internet self-efficacy and the psychology of the digital divide. Journal of Computer-Mediated Communication, 6(1), 0-0.

Elbert, N. J., van Os-Medendorp, H., van Renselaar, W., Ekeland, A. G., Hakkaart-van Roijen, L., Raat, H., Nijsten, T. E., \& Pasmans, S. G. (2014). Effectiveness and cost-effectiveness of eHealth interventions in somatic diseases: A systematic review of systematic reviews and meta-analyses. Journal of Medical Internet Research, 16(4), e110.

Elder, J. P., Ayala, G. X., \& Harris, S. (1999). Theories and intervention approaches to health-behavior change in primary care. American Journal of Preventive Medicine, 17(4), 275-284.

Elfeddali, I., Bolman, C., Candel, M., Wiers, R. W., \& de Vries, H. (2012). Preventing smoking relapse via webbased computer-tailored feedback: A randomized controlled trial. Journal of Medical Internet Research, 14(4), e109.

Elfeddali, I., Bolman, C., \& de Vries, H. (2012). SQ4U - a computer tailored smoking relapse prevention program incorporating planning strategy assignments and multiple feedback time points after the quitattempt: Development and design protocol. Contemporary Clinical Trials, 33(1), 151-158.

Elfeddali, I., Bolman, C., \& de Vries, H. (2013). Situational and affective risk situations of relapse and the quality of implementation intentions in an e-health smoking relapse prevention programme. Substance Use \& Misuse, 48(8), 635-644.

Eurostat. (2015, 22.12.2015). Internet use and activities. Retrieved from http://ec.europa.eu/eurostat/data/ database?node_code=isoc_bde15cua

Ewa, L. (2010). Digital divide-inequalities in level of implementation of new information and telecommunication technologies. Cross country study. Actas de la XII Reunión de la Economía Mundial.

Eysenbach, G. (2001). What is e-health? Journal of Medical Internet Research, 3(2), e20.

Eysenbach, G. (2005). The law of attrition. Journal of Medical Internet Research, 7(1), e11. 
Ezendam, N. P., Burg, J., Borsboom, G., van Empelen, P., \& Oenema, A. (2012). Differential effects of the computer-tailored FATaintPHAT programme on dietary behaviours according to sociodemographic, cognitive and home environmental factors. Public Health Nutrition, 17(2), 431-439.

Fennis, B. M., Adriaanse, M. A., Stroebe, W., \& Pol, B. (2011). Bridging the intention-behavior gap: Inducing implementation intentions through persuasive appeals. Journal of Consumer Psychology, 21(3), 302-311.

Ferguson, R., Gutberg, J., Schattke, K., Paulin, M., \& Jost, N. (2015). Self-determination theory, social media and charitable causes: An in-depth analysis of autonomous motivation: Self-determination theory, social media and charitable causes. European Journal of Social Psychology, 45(3), 298-307.

Fishbein, M., \& Ajzen, I. (2011). Predicting and changing behavior: The reasoned action approach (Vol. 1), New York: Taylor \& Francis.

Fleig, L., Pomp, S., Schwarzer, R., \& Lippke, S. (2013). Promoting exercise maintenance: How interventions with booster sessions improve long-term rehabilitation outcomes. Rehabilitation Psychology, 58(4), 323333.

Flycatcher Internet Research. (2015). Flycatcher Internet Research. Retrieved from http://www.flycatcher.eu/ eng/home/

Fox, J., \& Tang, W. Y. (2014). Sexism in online video games: The role of conformity to masculine norms and social dominance orientation. Computers in Human Behavior, 33, 314-320.

Fox, S. (2011). Health topics. Pew Research Center's Internet \& American Life Project. Retrieved from http://www.pewinternet.org/files/old-media//Files/Reports/2011/PIP_Health_Topics.pdf

Fox, S., \& Fallows, D. (2003). Internet health resources. Retrieved from http://www.pewinternet.org/ 2003/07/16/internet-health-resources/

Fry, J., \& Neff, R. (2009). Periodic prompts and reminders in health promotion and health behavior interventions: Systematic review. Journal of Medical Internet Research, 11, e16.

Gandek, B., Ware, J. E., Aaronson, N. K., Apolone, G., Bjorner, J. B., Brazier, J. E., Bullinger, M., Kaasa, S., Leplege, A., Prieto, L., \& Sullivan, M. (1998). Cross-validation of item selection and scoring for the SF-12 health survey in nine countries: Results from the IQOLA project. Journal of Clinical Epidemiology, 51(11), 1171-1178.

Garrett, J. J. (2010). Elements of user experience: The user-centered design for the web and beyond (2nd revised edition ed.), Berkeley: New Riders.

Gellert, P., Ziegelmann, J. P., Lippke, S., \& Schwarzer, R. (2012). Future time perspective and health behaviors: Temporal framing of self-regulatory processes in physical exercise and dietary behaviors. Annals of Behavioral Medicine, 43(2), 208-218.

Geraghty, A. W., Torres, L. D., Leykin, Y., Pérez-Stable, E. J., \& Muñoz, R. F. (2012). Understanding attrition from international Internet health interventions: A step towards global eHealth. Health Promotion International, 28(3), 442-452.

Geraghty, A. W., Wood, A. M., \& Hyland, M. E. (2010). Attrition from self-directed interventions: Investigating the relationship between psychological predictors, intervention content and dropout from a body dissatisfaction intervention. Social Science \& Medicine, 71(1), 30-37.

Gidlow, C., Johnston, L., Crone, D., Ellis, N., \& James, D. (2006). A systematic review of the relationship between socio-economic position and physical activity. Health Education Journal, 65(4), 338-367.

Gillholm, R., \& Gärling, T. (1997). Determinants of implementation intentions. Retrieved from http://utbildning.gu.se/digitalAssets/1286/1286088_gpr97_nr12.pdf

Godin, G., \& Kok, G. (1996). The Theory of Planned Behavior: A review of its applications to health-related behaviors. American Journal of Health Promotion, 11(2), 87-98.

Gollwitzer, P. M. (1993). Goal achievement: The role of intentions. European Review of Social Psychology, 4(1), 141-185.

Gollwitzer, P. M. (1999). Implementation intentions: Strong effects of simple plans. American Psychologist, 54(7), 493-503.

Gollwitzer, P. M., Odenthal, G., \& Wieber, F. (2010). Self-efficacy feelings moderate implementation intention effects. Self and Identity, 9(2), 177-194. 
Gollwitzer, P. M., \& Oettingen, G. (2013). Implementation intentions. In M. Gellman \& J. R. Turner (Eds.), Encyclopedia of Behavioral Medicine (pp. 1043-1048). New York: Springer-Verlag.

Gollwitzer, P. M., Sheeran, P., \& Mark, P. Z. (2006). Implementation intentions and goal achievement: A metaanalysis of effects and processes. Advances in Experimental Social Psychology, Volume 38, 69-119.

Griffiths, F., Lindenmeyer, A., Powell, J., Lowe, P., \& Thorogood, M. (2006). Why are health care interventions delivered over the Internet? A systematic review of the published literature. Journal of Medical Internet Research, 8(2), e10.

Grittner, U., Kuntsche, S., Gmel, G., \& Bloomfield, K. (2013). Alcohol consumption and social inequality at the individual and country levels--results from an international study. European Journal of Public Health, 23(2), 332-339.

Griva, F., Tseferidi, S. I., \& Anagnostopoulos, F. (2015). Time to get healthy: Associations of time perspective with perceived health status and health behaviors. Psychology, Health \& Medicine, 20(1), 25-33.

Groot, W., \& Maassen van den Brink, M. (2006). What does education do to our health? Copenhagen symposium on measuring the effects of education on health and civic engagement. Retrieved from http://www.oecd.org/edu/innovation-education/37425763.pdf

Grube, J. W., Morgan, M., \& McGree, S. T. (1986). Attitudes and normative beliefs as predictors of smoking intentions and behaviours: A test of three models. British Journal of Social Psychology, 25(2), 81-93.

Guthrie, L. C., Butler, S. C., Lessl, K., Ochi, O., \& Ward, M. M. (2014). Time perspective and exercise, obesity and smoking: Moderation of associations by age. American Journal of Health Promotion, 29(1), 9-16.

Habibović, M., Cuijpers, P., Alings, M., van der Voort, P., Theuns, D., Bouwels, L., Herrman, J.-P., Valk, S., \& Pedersen, S. (2014). Attrition and adherence in a web-based distress management program for implantable cardioverter defibrillator patients (WEBCARE): Randomized controlled trial. Journal of Medical Internet Research, 16(2), e52.

Hagger, M. S., \& Luszczynska, A. (2014). Implementation intention and action planning interventions in health contexts: State of the research and proposals for the way forward. Applied Psychology: Health and WellBeing, 6(1), 1-47.

Hainey, T., Connolly, T., Stansfield, M., \& Boyle, E. (2011). The differences in motivations of online game players and offline game players: A combined analysis of three studies at higher education level. Computers \& Education, 57(4), 2197-2211.

Hall, A. K., \& Marston, H. R. (2021). Gaming and older adults in the digital age of healthcare. In J. Hoof, G. Demiris, \& J. M. E. Wouters (Eds.), Handbook of Smart Homes, Health Care and Well-Being (pp. 1-9). Cham: Springer International Publishing.

Hall, M. G., Ribisl, K. M., \& Brewer, N. T. (2014). Smokers' and nonsmokers' beliefs about harmful tobacco constituents: Implications for FDA communication efforts. Nicotine \& Tobacco Research, 16(3), 343-350.

Hamel, L. M., \& Robbins, L. B. (2013). Computer- and web-based interventions to promote healthy eating among children and adolescents: A systematic review. Journal of Advanced Nursing, 69(1), 16-30.

Hamilton, W. L., Norton, G. d., Ouellette, T. K., Rhodes, W. M., Kling, R., \& Connolly, G. N. (2004). Smokers' responses to advertisements for regular and light cigarettes and potential reduced-exposure tobacco products. Nicotine \& Tobacco Research, 6(3), 353-362.

Hammond, D. (2011). Health warning messages on tobacco products: A review. Tobacco Control, 20(5), 327337.

Hanson, M. D., \& Chen, E. (2007). Socioeconomic status and health behaviors in adolescence: A review of the literature. Journal of Behavioral Medicine, 30(3), 263-285.

Hargittai, E. (2002). Second-level digital divide: Differences in people's online skills. First Monday, 7(4).

Hargittai, E. (2010). Digital Na(t)ives? Variation in Internet skills and uses among members of the "Net Generation". Sociological Inquiry, 80(1), 92-113.

Hargittai, E., \& Hinnant, A. (2008). Digital inequality differences in young adults' use of the Internet. Communication Research, 35(5), 602-621.

Hargittai, E., \& Shafer, S. (2006). Differences in actual and perceived online skills: The role of gender. Social Science Quarterly, 87(2), 432-448. 
Hargittai, E., \& Young, H. (2012). Searching for a "Plan B": Young adults' strategies for finding information about emergency contraception online. Policy \& Internet, 4(2), 1-23.

Hawkins, R. P., Kreuter, M., Resnicow, K., Fishbein, M., \& Dijkstra, A. (2008). Understanding tailoring in communicating about health. Health Education Research, 23(3), 454-466.

Heck, R. H., Thomas, S., \& Tabata, L. (2012). Multilevel modeling of categorical outcomes using IBM SPSS. New York: Taylor \& Francis Ltd.

Hibbard, J. H., \& Greene, J. (2013). What the evidence shows about patient activation: Better health outcomes and care experiences; fewer data on costs. Health Affairs, 32(2), 207-214.

Higgins, J. P. T., \& Thompson, S. G. (2002). Quantifying heterogeneity in a meta-analysis. Statistics in Medicine, 21(11), 1539-1558.

Hiscock, R., Bauld, L., Amos, A., Fidler, J. A., \& Munafò, M. (2012). Socioeconomic status and smoking: A review. Annals of the New York Academy of Sciences, 1248(1), 107-123.

Hoeymans, N., van Loon, A., van den Berg, M., Harbers, M., Hilderink, H., van Oers, J., \& Schoemaker, C. (2014). A healthier Netherlands. Key findings from the Dutch 2014 public health status and foresight report. Retrieved from http://www.eengezondernederland.nl/dsresource?type=pdf\&object $i d=7$ ead4f11-939c-49e3-9cb1-8bac7d3b7c76\&versionid=\&subobjectname=

Howard, P. E. N., Rainie, L., \& Jones, S. (2001). Days and nights on the Internet: The impact of a diffusing technology. American Behavioral Scientist, 45(3), 383-404.

Huedo-Medina, T. B., Sánchez-Meca, J., Marín-Martínez, F., \& Botella, J. (2006). Assessing heterogeneity in meta-analysis: Q statistic or 12 index? Psychological Methods, 11(2), 193.

Hulshof, K. F. A. M., Brussaard, J. H., Kruizinga, A. G., Telman, J., \& Löwik, M. R. H. (2003). Socio-economic status, dietary intake and 10 y trends: The Dutch National Food Consumption Survey. European Journal of Clinical Nutrition, 57(1), 128-137.

Information Solutions Group. (2011). PopCap games social gaming research. Retrieved from http://www.info solutionsgroup.com/pdfs/2011_PopCap_Social_Gaming_Research_Results.pdf

Information Solutions Group. (2013). PopCap games mobile Gaming research. Retrieved from http://www.infosolutionsgroup.com/popcapmobile2013.pdf

Ipsos-Eureka. (2009). Public health value of disclosed cigarette ingredients and emissions data. Retrieved from http://www.ipsos.com/public-affairs/sites/www.ipsos.com.public-affairs/files/documents/Australiatobacco-emissions.pdf

Ivory, J. D. (2006). Still a man's game: Gender representation in online reviews of video games. Mass Communication and Society, 9(1), 103-114.

Janssen, E., van Osch, L., Lechner, L., Candel, M., \& de Vries, H. (2011). Thinking versus feeling: Differentiating between cognitive and affective components of perceived cancer risk. Psychology \& Health, 27(7), 767783.

Janz, N. K., \& Becker, M. H. (1984). The Health Belief Model: A decade later. Health Education \& Behavior, 11(1), 1-47.

Jensen, J. D., King, A. J., Davis, L. A., \& Guntzviller, L. M. (2010). Utilization of Internet technology by lowincome adults: The role of health literacy, health numeracy, and computer assistance. Journal of Aging and Health, 22(6), 804-826.

Jones, R. B., Ashurst, E. J., Atkey, J., \& Duffy, B. (2015). Older people going online: Its value and before-after evaluation of volunteer support. Journal of Medical Internet Research, 17(5), e122.

Jones, S., \& Fox, S. (2009). Generations online in 2009. Retrieved from http://www.pewinternet.org/ 2009/01/28/generations-online-in-2009/

Kalba, K. (2000). Only the lonely: Internet usage and effects: A review of Internet and society. A preliminary report, Norman H. Nie \& Lutz Erbring, Stanford Institute for the Quantitative Study of Society. Telecommunications Policy, 24(6), 621-623.

Kalichman, S. C., Cherry, C., Cain, D., Weinhardt, L. S., Benotsch, E., Pope, H., \& Kalichman, M. (2006). Health information on the Internet and people living with HIV/AIDS: Information evaluation and coping styles. Health Psychology, 25(2), 205. 
Kelders, S. M., Kok, R. N., Ossebaard, H. C., \& Van Gemert-Pijnen, J. E. W. C. (2012). Persuasive system design does matter: A systematic review of adherence to web-based interventions. Journal of Medical Internet Research, 14(6), e152.

Kickbusch, I. S. (2001). Health literacy: Addressing the health and education divide. Health Promotion International, 16(3), 289-297.

Kiecolt-Glaser, J. K., \& Newton, T. L. (2001). Marriage and health: His and hers. Psychological Bulletin, 127(4), 472-503.

Kienhuis, A., Schenk, E., \& Talhout, R. ( 2013). Publieksinformatie over additieven in tabaksproducten, the right to know. Retrieved from

http://www.rivm.nl/Documenten_en_publicaties/Wetenschappelijk/Rapporten/2013/mei/Publieksinfor matie_over_additieven_in_tabaksproducten_the_right_to_know

Kivits, J. (2006). Informed patients and the Internet: A mediated context for consultations with health professionals. Journal of Health Psychology, 11(2), 269-282.

Klasnja, P., \& Pratt, W. (2012). Healthcare in the pocket: Mapping the space of mobile-phone health interventions. Journal of Biomedical Informatics, 45(1), 184-198.

Kohl, L. F. M., Crutzen, R., \& de Vries, N. K. (2013). Online prevention aimed at lifestyle behaviors: A systematic review of reviews. Journal of Medical Internet Research, 15(7), e146.

Koivusilta, L., Lintonen, T., \& Rimpela, A. (2007). Orientations in adolescent use of information and communication technology: A digital divide by sociodemographic background, educational career, and health. Scandinavian Journal of Public Health, 35(1), 95-103.

Kok, G., Gottlieb, N. H., Peters, G.-J. Y., Mullen, P. D., Parcel, G. S., Ruiter, R. A. C., Fernández, M. E., Markham, C., \& Bartholomew, L. K. (2016). A taxonomy of behavior change methods: An Intervention Mapping approach. Health Psychology Review, 10(3), 297-312.

Konstantinidis, E., Billis, A., Mouzakidis, C., Zilidou, V., Antoniou, P., \& Bamidis, P. (2014). Design, implementation and wide pilot deployment of FitForAll: An easy to use exergaming platform improving physical fitness and life quality of senior citizens. Journal of Biomedical and Health Informatics, 20(1), 189-200.

Koster, A., Bosma, H., Broese van Groenou, M. I., Kempen, G. I., Penninx, B. W., van Eijk, J. T., \& Deeg, D. J. (2006). Explanations of socioeconomic differences in changes in physical function in older adults: Results from the Longitudinal Aging Study Amsterdam. BMC Public Health, 6(1), 1-12.

Kraft, P., \& Yardley, L. (2009). Current issues and new directions in Psychology and Health: What is the future of digital interventions for health behaviour change? Psychology \& Health, 24(6), 615-618.

Krebs, P., Prochaska, J. O., \& Rossi, J. S. (2010). A meta-analysis of computer-tailored interventions for health behavior change. Preventive Medicine, 51(3-4), 214-221.

Kreuter, M., Farrell, D., Olevitch, L., \& Brennan, L. (2000). Tailoring health messages. Customizing communication with computer technology. Mahwah, New Jersey: Lawrence Erlbaum Associates.

Kroeze, W., Werkman, A., \& Brug, J. (2006). A systematic review of randomized trials on the effectiveness of computer-tailored education on physical activity and dietary behaviors. Annals of Behavioral Medicine, 31(3), 205-223.

Krumpal, I. (2013). Determinants of social desirability bias in sensitive surveys: A literature review. Quality \& Quantity, 47(4), 2025-2047.

Lachman, M. E., Neupert, S. D., \& Agrigoroaei, S. (2011). The relevance of control beliefs for health and aging. In K. W. Schaie \& S. L. Willis (Eds.), Handbook of the Psychology of Aging (Vol. 7). Seattle: Academic Press.

Lachman, M. E., \& Weaver, S. L. (1998). The sense of control as a moderator of social class differences in health and well-being. Journal of Personality and Social Psychology, 74(3), 763-773.

Lahelma, E., Laaksonen, M., Martinkainen, P., \& Rahkonen, O. (2008). Die Mehrdimensionalität der sozioökonomischen Lage -Konsequenzen für die Analyse gesundheitlicher Ungleichheit. In U. Bauer, B. U. H, \& M. Richter (Eds.), Health inequalities. Determinanten und Mechanismen gesundheitlicher Ungleichheit. Wiesbaden: Verlag für Sozialwissenschaften. 
Lampert, T., Kroll, L. E., von der Lippe, E., Müters, S., \& Stolzenberg, H. (2013). Socioeconomic status and health: Results of the German Health Interview and Examination Survey for adults (DEGS1). Bundesgesundheitsblatt - Gesundheitsforschung - Gesundheitsschutz, 56(5-6).

Laugesen, J., Hassanein, K., \& Yuan, Y. F. (2015). The impact of Internet health information on patient compliance: A research model and an empirical study. Journal of Medical Internet Research, 17(6), e143.

Lemmens, P., Tan, E. S., \& Knibbe, R. A. (1992). Measuring quantity and frequency of drinking in a general population survey: A comparison of five indices. Journal of Studies on Alcohol and Drugs, 53(5), 476.

Leung, L., \& Lee, P. S. (2005). Multiple determinants of life quality: The roles of Internet activities, use of new media, social support, and leisure activities. Telematics and Informatics, 22(3), 161-180.

Li, L. (2010). A critical review of technology acceptance literature. Retrieved from http://www.swdsi.org/swdsi2010/sw2010_preceedings/papers/pa104.pdf

Lippke, S., Ziegelmann, J. P., \& Schwarzer, R. (2004). Behavioral intentions and action plans promote physical exercise: A longitudinal study with orthopedic rehabilitation patients. Journal of Sport \& Exercise Psychology, 26(3), 470-483.

Lippke, S., Ziegelmann, J. P., Schwarzer, R., \& Velicer, W. F. (2009). Validity of stage assessment in the adoption and maintenance of physical activity and fruit and vegetable consumption. Health Psychology, 28(2), 183-193.

Livingstone, S. (2004). Media literacy and the challenge of new information and communication technologies. The Communication Review, 7(1), 3-14.

Livingstone, S., Bober, M., \& Helsper, E. (2005). Internet literacy among children and young people: Findings from the UK Children Go Online Project (4). Retrieved from http://eprints.Ise.ac.uk/397/

Livingstone, S., \& Helsper, E. (2007). Gradations in digital inclusion: Children, young people and the digital divide. New Media \& Society, 9(4), 671-696.

Lofgren, K. (2015). 2015 Video game statistics \& trends who's playing what \& why? Retrieved from http://www.bigfishgames.com/blog/2015-global-video-game-stats-whos-playing-what-and-why/

Luijkx, R., \& de Heus, M. (2008). The educational system of the Netherlands In: The international standard classification of education (ISCED-97): An evaluation of content and criterion validity for 15 European countries (pp. 47-75). Mannheim: Mannheimer Zentrum für Europäische Sozialforschung (MZES).

Luo, M. M., \& Remus, W. (2014). Uses and gratifications and acceptance of web-based information services: An integrated model. Computers in Human Behavior, 38, 281-295.

Lustria, M. L. A., Noar, S. M., Cortese, J., Van Stee, S. K., Glueckauf, R. L., \& Lee, J. (2013). A meta-analysis of Web-delivered tailored health behavior change interventions. Journal of Health Communication, 18(9), $1-31$.

Luszczynska, A., \& Sutton, S. (2006). Physical activity after cardiac rehabilitation: Evidence that different types of self-efficacy are important in maintainers and relapsers. Rehabilitation Psychology, 51(4), 314-321.

Mackenbach, J. P. (2006). Health inequalities: Europe in profile. Retrieved from http://www.who.int/ social_determinants/resources/european_inequalities.pdf

Mackenbach, J. P., Stirbu, I., Roskam, A.-J. R., Schaap, M. M., Menvielle, G., Leinsalu, M., \& Kunst, A. E. (2008). Socioeconomic inequalities in health in 22 European countries. New England Journal of Medicine, 358(23), 2468-2481.

Madden, M., \& Rainie, L. (2003). America's online pursuits: The changing picture of who's online and what they do: Pew Internet \& American Life Project.

Mäder, U. R. S., Martin, B. W., Schutz, Y., \& Martil, B. (2006). Validity of four short physical activity questionnaires in middle-aged persons. Medicine \& Science in Sports \& Exercise, 38(7), 1255-1266.

Mahon, N. E., Yarcheski, T. J., \& Yarcheski, A. (1997). Future time perspective and positive health practices in young adults: An extension. Perceptual and Motor Skills, 84(3 Pt 2), 1299-1304.

Margolis, R. (2013). Educational differences in healthy behavior changes and adherence among middle-aged Americans. Journal of Health and Social Behavior, 54(3), 353-368.

Martin, S. P., \& Robinson, J. P. (2007). The income digital divide: Trends and predictions for levels of Internet use. Social Problems, 54(1), 1-22. 
McClure, J. B., Shortreed, S. M., Bogart, A., Derry, H., Riggs, K., St John, J., Nair, V., \& An, L. (2013). The effect of program design on engagement with an Internet-based smoking intervention: Randomized factorial trial. Journal of Medical Internet Research, 15(3), e69.

McGuire, W. J. (1989). Theoretical foundations of campaigns. In R. E. Rice \& C. K. Atkin (Eds.), Public communication campaigns (2nd ed.). Newbury Park: Sage Publications.

McKay, H. G., Danaher, B. G., Seeley, J. R., Lichtenstein, E., \& Gau, J. M. (2008). Comparing two web-based smoking cessation programs: Randomized controlled trial. Journal of Medical Internet Research, 10(5), e40.

McMullan, M. (2006). Patients using the Internet to obtain health information: How this affects the patienthealth professional relationship. Patient Education and Counseling, 63(1-2), 24-28.

McQuail, D. (2010). McQuail's mass communication theory (Vol. 6th Revised edition): Sage publications Ltd.

Melville, K. M., Casey, L. M., \& Kavanagh, D. J. (2010). Dropout from Internet-based treatment for psychological disorders. British Journal of Psychology, 49(Pt 4), 455-471.

Merckel, C., \& Pragst, F. (2007). Tabakzusatzstoffe in Zigaretten - Verwendungszweck und Gefahrenpotential. Journal für Verbraucherschutz und Lebensmittelsicherheit, 2(3), 287-301.

Merletti, F., Galassi, C., \& Spadea, T. (2011). The socioeconomic determinants of cancer. Environmental Health, 10(Suppl 1), S7.

Mesch, G., Mano, R., \& Tsamir, J. (2012). Minority status and health information search: A test of the social diversification hypothesis. Social Science \& Medicine, 75(5), 854-858.

Miniwatts Marketing Group. (2015, 27.11.2015). Internet users in the European Union - 2015. Retrieved from http://www.internetworldstats.com/stats9.htm\#eu

Mitchell, M. E., Lebow, J. R., Uribe, R., Grathouse, H., \& Shoger, W. (2011). Internet use, happiness, social support and introversion: A more fine grained analysis of person variables and Internet activity. Computers in Human Behavior, 27(5), 1857-1861.

Močnik, D., \& Širec, K. (2010). The determinants of Internet use controlling for income level: Cross-country empirical evidence. Information Economics and Policy, 22(3), 243-256.

Monteiro, C. A., Moura, E. C., Conde, W. L., \& Popkin, B. M. (2004). Socioeconomic status and obesity in adult populations of developing countries: A review. Bull World Health Organ, 82, 940-946.

Moroshko, I., Brennan, L., \& O'Brien, P. (2011). Predictors of dropout in weight loss interventions: A systematic review of the literature. Obesity Reviews, 12(11), 912-934.

Mudde, A. N., Willemsen, M. C., Kremers, S., \& de Vries, H. (2006). Measuring instruments for research regarding smoking and smoking cessation [Meetinstrumenten voor onderzoek naar roken en stoppen met roken.]. The Hague: STIVORO.

Murray, A. D., Staff, R. T., McNeil, C. J., Salarirad, S., Starr, J. M., Deary, I. J., \& Whalley, L. J. (2012). Brain lesions, hypertension and cognitive ageing in the 1921 and 1936 Aberdeen birth cohorts. AGE, 34(2), 451-459.

Murray, E., White, I. R., Varagunam, M., Godfrey, C., Khadjesari, Z., \& McCambridge, J. (2013). Attrition revisited: Adherence and retention in a web-based alcohol trial. Journal of Medical Internet Research, 15(8), e162.

Murray, T. C., Rodgers, W. M., \& Fraser, S. N. (2012). Exploring the relationship between socioeconomic status, control beliefs and exercise behavior: A multiple mediator model. Journal of Behavioral Medicine, 35(1), 63-73.

Naimi, T. S., Brewer, R. D., Mokdad, A., Denny, C., Serdula, M. K., \& Marks, J. S. (2003). Binge drinking among US adults. JAMA, 289(1), 70-75.

Nam, S. K., \& Lee, S. M. (2015). The role of attachment and stigma in the relationship between stress and attitudes toward counseling in South Korea. Journal of Counseling \& Development, 93(2), 212-224.

Neter, E., \& Brainin, E. (2012). eHealth literacy: Extending the digital divide to the realm of health information. Journal of Medical Internet Research, 14(1), e19.

Neve, M., Collins, C. E., \& Morgan, P. J. (2010). Dropout, nonusage attrition, and pretreatment predictors of nonusage attrition in a commercial web-based weight loss program. Journal of Medical Internet Research, 12(4), e69. 
Neve, M., Morgan, P. J., Jones, P. R., \& Collins, C. E. (2010). Effectiveness of web-based interventions in achieving weight loss and weight loss maintenance in overweight and obese adults: A systematic review with meta-analysis. Obesity Reviews, 11(4), 306-321.

Neville, L. M., O'Hara, B., \& Milat, A. J. (2009). Computer-tailored dietary behaviour change interventions: A systematic review. Health Education Research, 24(4), 699-720.

Nie, N. H., Hillygus, D. S., \& Erbring, L. (2002). Internet use, interpersonal relations, and sociability. In Wellman, B., \& Haythornthawaite, C (Eds.), The Internet in everyday life. Malden: Blackwell Publishers Ltd.

Nigg, C. R. (2005). There is more to stages of exercise than just exercise. Exercise and Sport Sciences Reviews, 33(1), 32-35.

Noar, S., Benac, C., \& Harris, M. (2007). Does tailoring matter? Meta-analytic review of tailored print health behavior change interventions. Psychological Bulletin, 133(4), 673-693.

Noar, S., \& Harrington, N. (2012). eHealth applications. New York: Routledge.

Noar, S. M., Grant Harrington, N., van Stee, S. K., \& Shemanski Aldrich, R. (2011). Tailored health communication to change lifestyle behaviors. American Journal of Lifestyle Medicine, 5(2), 112-122.

Noguchi, K., Albarracín, D., Durantini, M. R., \& Glasman, L. R. (2007). Who participates in which health promotion programs? A meta-analysis of motivations underlying enrollment and retention in HIVprevention interventions. Psychological Bulletin, 133(6), 955-975.

Norman, C. D., \& Skinner, H. A. (2006). eHealth literacy: Essential skills for consumer health in a networked world. Journal of Medical Internet Research, 8(2), e9.

Norman, G. J., Zabinski, M. F., Adams, M. A., Rosenberg, D. E., Yaroch, A. L., \& Atienza, A. A. (2007). A review of e-health interventions for physical activity and dietary behaviour change. American Journal of Preventive Medicine, 33(4), 336-345.

O'Connor, R. J., Hyland, A., Giovino, G. A., Fong, G. T., \& Cummings, K. M. (2005). Smoker awareness of and beliefs about supposedly less-harmful tobacco products. American Journal of Preventive Medicine, 29(2), 85-90.

Olphert, W., \& Damodaran, L. (2013). Older people and digital disengagement: A fourth digital divide? Gerontology, 59(6), 564-570.

Orbell, S., \& Sheeran, P. (1998). 'Inclined abstainers': A problem for predicting health-related behaviour. Britsh Journal of Social Psychology, 37, 151-165.

Organisation for Economic Co-operation and Development. (2000). Literacy in the Information Age. Final report of the international adult literacy survey. Retrieved from http://www.oecd.org/education/ skillsbeyond-school/41529765.pdf

Paasche-Orlow, M. K., Parker, R. M., Gazmararian, J. A., Nielsen-Bohlman, L. T., \& Rudd, R. R. (2005). The prevalence of limited health literacy. Journal of General Internal Medicine, 20(2), 175-184.

Pampel, F. C., Krueger, P. M., \& Denney, J. T. (2010). Socioeconomic disparities in health behaviors. Annual Review of Sociology, 36, 349.

Pang, P. C. I., Chang, S., Verspoor, K., \& Pearce, J. (2016). Designing health websites based on users' webbased information-seeking behaviors: A mixed-method observational study. Journal of Medical Internet Research, 18(6), e145.

Parascandola, M., Augustson, E., \& Rose, A. (2009). Characteristics of current and recent former smokers associated with the use of new potential reduced-exposure tobacco products. Nicotine \& Tobacco Research, 11(12), 1431-1438.

Parks-Stamm, E. J., Gollwitzer, P. M., \& Oettingen, G. (2007). Action control by implementation intentions: Effective cue detection and efficient response initiation. Social Cognition, 25(2), 248-266.

Peel, D. A., Bolman, C., Golsteijn, R. H. J., de Vries, H., Mudde, A. N., van Stralen, M. M., \& Lechner, L. (2012). Differences in reach and attrition between web-based and print-delivered tailored interventions among adults over 50 years of age: Clustered randomized trial. Journal of Medical Internet Research, 14(6), e179.

Peels, D. A., van Stralen, M. M., Bolman, C., Golsteijn, R. H. J., de Vries, H., Mudde, A. N., \& Lechner, L. (2014). The differentiated effectiveness of a printed versus a web-based tailored physical activity intervention among adults aged over 50. Health Education Research, 29(5), 870-882. 
Peters, G.-J. Y. (2014). The alpha and the omega of scale reliability and validity. The European Health Psychologist, 16(2), 56-69.

Poirier, J., \& Cobb, N. K. (2012). Social influence as a driver of engagement in a web-based health intervention. Journal of Medical Internet Research, 14(1), e36.

Poortinga, W., Dunstan, F. D., \& Fone, D. L. (2008). Health locus of control beliefs and socio-economic differences in self-rated health. Preventive Medicine, 46(4), 374-380.

Poos, M., Burggink, J., \& van der Lucht, F. (2014). Levensverwachting: Zijn er verschillen naar sociaaleconomische status? Volksgezondheid Toekomst Verkenning, Nationaal Kompas Volksgezondheid. Retrieved from http://www.nationaalkompas.nl/gezondheid-en-ziekte/sterfte-levensverwachting-endaly-s/levens verwachting/verschillen-sociaaleconomisch/

Postel, M. G., de Haan, H. A., ter Huurne, E. D., van der Palen, J., Becker, E. S., \& de Jong, C. A. J. (2011). Attrition in web-based treatment for problem drinkers. Journal of Medical Internet Research, 13(4), e117.

Postel, M. G., de Jong, C. A. J., de Haan, H. A., Becker, E. S., \& ter Huurne, E. D. (2010). Effectiveness of a webbased intervention for problem drinkers and reasons for dropout: Randomized controlled trial. Journal of Medical Internet Research, 12(4), e68.

Price, M., Gros, D. F., McCauley, J. L., Gros, K. S., \& Ruggiero, K. J. (2012). Nonuse and dropout attrition for a web-based mental health intervention delivered in a post-disaster context. Psychiatry, 75(3), 267.

Rabinoff, M., Caskey, N., Rissling, A., \& Park, C. (2007). Pharmacological and chemical effects of cigarette additives. American Journal of Public Health, 97(11), 1981-1991.

Rafferty, A. P., Anderson, J. V., McGee, H. B., \& Miller, C. E. (2002). A healthy diet indicator: Quantifying compliance with the dietary guidelines using the BRFSS. Preventive Medicine, 35(1), 9-15.

Reinwand, D. A., Crutzen, R., Elfeddali, I., Scheider, F., Schulz, D. N., Stanczyk, N. E., Smit, E. S., Tange, H., Voncken-Brewster, V., Walthouwer, M. J., Hoving, C., \& de Vries, H. (2015). Impact of educational level on study attrition and evaluation of web-based computer-tailored interventions: Results from seven randomized controlled trials. Journal of Medical Internet Research, 17(10), e228.

Reinwand, D. A., Crutzen, R., Storm, V., Wienert, J., Kuhlmann, T., de Vries, H., \& Lippke, S. (2016). Generating and predicting high quality action plans to facilitate physical activity and fruit and vegetable consumption: Results from an experimental arm of a randomised controlled trial. BMC Public Health, 16(1), 317.

Reinwand, D. A., Kuhlmann, T., Wienert, J., de Vries, H., \& Lippke, S. (2013). Designing a theory- and evidencebased tailored eHealth rehabilitation aftercare program in Germany and the Netherlands: Study protocol. BMC Public Health, 13(1), 1081.

Reinwand, D. A., Schulz, D. N., Crutzen, R., Kremers, S. P. J., \& de Vries, H. (2015). Who follows eHealth interventions as recommended? A study of participants' personal characteristics from the experimental arm of a randomized controlled trial. Journal of Medical Internet Research, 17(5), e115.

Riper, H., Kramer, J., Keuken, Smit, F., Schippers, G., \& Cuijpers, P. (2008). Predicting successful treatment outcome of web-based self-help for problem drinkers: Secondary analysis from a randomized controlled trial. Journal of Medical Internet Research, 10(4), e46.

Riper, H., Spek, V., Boon, B., Conijn, B., Kramer, J., Martin-Abello, K., \& Smit, F. (2011). Effectiveness of e-selfhelp interventions for curbing adult problem drinking: A meta-analysis. Journal of Medical Internet Research, 13(2), e42.

Ritterband, L., \& Thorndike, F. (2006). Internet interventions or patient education web sites? Journal of Medical Internet Research, 8(3), e18; author reply e19.

Ritterband, L. M., Gonder-Frederick, L. A., Cox, D. J., Clifton, A. D., West, R. W., \& Borowitz, S. M. (2003). Internet interventions: In review, in use, and into the future. Professional Psychology: Research and Practice, 34(5), 527-534.

RIVM. (2005). Lokale en nationale Monitor gezondheid. Indicatoren voor de Monitor Volksgezondheid. Retrieved from https://www.monitorgezondheid.nl/LNMV.xml

RIVM. (2015). EMTOC. Retrieved from http://www.rivm.nl/en/Topics/T/Tobacco/EMTOC 
Rodrigues, A. M., O'Brien, N., French, D. P., Glidewell, L., \& Sniehotta, F. (2015). The question-behavior effect: Genuine effect or spurious phenomenon? A systematic review of randomized controlled trials with meta-analyses. Health Psychology, 34(1), 61-78.

Rogers, E. M. (2003). Diffusion of Innovations (5 ed.). New York: Free Press.

Rooke, S., Thorsteinsson, E., Karpin, A., Copeland, J., \& Allsop, D. (2010). Computer-delivered interventions for alcohol and tobacco use: A meta-analysis. Addiction (Abingdon, England), 105(8), 1381-1390.

Ruggiero, T. E. (2000). Uses and gratifications theory in the 21st century. Mass Communication and Society, 3(1), 3-37.

Ryan, R. M., \& Deci, E. L. (2000). Self-determination theory and the facilitation of intrinsic motivation, social development, and well-being. American Psychologist, 55, 68-78.

Savage, C. (2012). Alcohol and tobacco related health inequity: A population health perspective. Journal of Addictions Nursing, 23(1), 72-74.

Schenk, E., van de Nobelen, S., Pennings, J., Kienhuis, A., \& Talhout, R. (2015). Additieven in Nederlandse tabaksproducten. Trendanalyse gegevens 2010-2013. Retrieved from http://www.rivm.nl/ dsresource?objectid=rivmp:277609\&type=org\&disposition=inline

Scholz, U., Sniehotta, F., Schüz, B., \& Oeberst, A. (2007). Dynamics in self-regulation: Plan execution selfefficacy and mastery of action plans. Journal of Applied Social Psychology, 37(11), 2706-2725.

Schulz, D. N., Candel, M. J., Kremers, S., Reinwand, D. A., Jander, A., \& de Vries, H. (2013). Effects of a webbased tailored intervention to reduce alcohol consumption in adults: Randomized controlled trial. Journal of Medical Internet Research, 15(9), e206.

Schulz, D. N., Kremers, S. P. J., van Osch, L., Schneider, F., van Adrichem, M. J. G., \& de Vries, H. (2011). Testing a Dutch web-based tailored lifestyle programme among adults: A study protocol. BMC Public Health, 11(1), 108.

Schulz, D. N., Kremers, S. P. J., Vandelanotte, C., van Adrichem, M. J. G., Schneider, F., Candel, M. J. J. M., \& de Vries, H. (2014). Effects of a web-based tailored multiple-lifestyle intervention for adults: A two-year randomized controlled trial comparing sequential and simultaneous delivery modes. Journal of Medical Internet Research, 16(1), e26.

Schulz, D. N., Schneider, F., de Vries, H., van Osch, L., van Nierop, P. W. M., \& Kremers, S. P. J. (2012). Program completion of a web-based tailored lifestyle intervention for adults: Differences between a sequential and a simultaneous approach. Journal of Medical Internet Research, 14(2), e26.

Schulz, D. N., Smit, E. S., Stanczyk, N. E., Kremers, S., de Vries, H., \& Evers, S. M. A. A. (2014). Economic evaluation of a web-based tailored lifestyle intervention for adults: Findings regarding cost-effectiveness and cost-utility from a randomized controlled trial. Journal of Medical Internet Research, 16(3), e91.

Schulz, P. J., \& Nakamoto, K. (2013). Health literacy and patient empowerment in health communication: The importance of separating conjoined twins. Patient Education and Counseling, 90(1), 4-11.

Schwarzer, R. (1992). Self-efficacy in the adoption and maintenance of health behaviors: Theoretical approaches and a new model. In R. Schwarzer (Ed.), Self-efficacy: Thought control of action. (pp. 217243). Washington, DC: Hemisphere Publishing Corp.

Schwarzer, R. (2004). Psychologie des Gesundheitsverhaltens. Einführung in die Gesundheitspsychologie (Vol. 3). Göttingen: Hogrefe.

Schwarzer, R. (2008). Modeling health behavior change: How to predict and modify the adoption and maintenance of health behaviors. Applied Psychology: An International Review, 57(1), 1-29.

Schwarzer, R., Lippke, S., \& Luszczynska, A. (2011). Mechanisms of health behavior change in persons with chronic illness or disability: The Health Action Process Approach (HAPA). Rehabilitation Psychology, 56(3), 161-170.

Schwarzer, R., Luszczynska, A., Ziegelmann, J. P., Scholz, U., \& Lippke, S. (2008). Social-cognitive predictors of physical exercise adherence: Three longitudinal studies in rehabilitation. Health Psychology, 27(1, Suppl), S54-S63.

Schweier, R., Romppel, M., Richter, C., Hoberg, E., Hahmann, H., Scherwinski, I., Kosmützky, G., \& Grande, G. (2014). A web-based peer-modeling intervention aimed at lifestyle changes in patients with coronary 
heart disease and chronic back pain: Sequential controlled trial. Journal of Medical Internet Research, 16(7), e177.

Schwerpenzeel, A. C., \& Das, M. (2010). "True" longitudinal and probability-based Internet panels: Evidence from the Netherlands. In M. Das, P. Ester, \& L. Kaczmirek (Eds.), Social and behavioral research and the Internet: Advances in applied methods and research strategies. (pp. 77-104): Taylor \& Francis.

Scoreboard. (2013). Internet use and skills. Retrieved from https://ec.europa.eu/digital-agenda/sites/digitalagenda/files/DAE\%20SCOREBOARD\%202013\%20-\%203-INTERNET\%20USE\%20AND\%20SKILLS.pdf

Sheeran, P. (2002). Intention-behavior relations: A conceptual and empirical review. European Review of Social Psychology, 12(1), 1-36.

Shiffman, S., Pillitteri, J. L., Burton, S. L., \& Di Marino, M. E. (2004). Smoker and ex-smoker reactions to cigarettes claiming reduced risk. Tobacco Control, 13(1), 78-84.

Skelton, J. A., Irby, M. B., \& Geiger, A. M. (2014). A systematic review of satisfaction and pediatric obesity treatment: New avenues for addressing attrition. Journal for Healthcare Quality: Official Publication of the National Association for Healthcare Quality, 36(4), 5-22.

Smeets, T., Kremers, S., De Vries, H., \& Brug, J. (2007). Effects of tailored feedback on multiple health behaviors. Annals of Behavioral Medicine, 33, 117-123.

Smit, E. S., Candel, M. J. J. M., Hoving, C., \& De Vries, H. (2016). Results of the PAS study: A randomized controlled trial evaluating the effectiveness of a web-based multiple tailored smoking cessation program combined with tailored counselling by practice nurses. Health Communication, 31(9), 1165-1173.

Smit, E. S., de Vries, H., \& Hoving, C. (2010). The PAS study: A randomized controlled trial evaluating the effectiveness of a web-based multiple tailored smoking cessation program and tailored counselling by practice nurses. Contemporary Clinical Trials, 31(3), 251-258.

Smit, E. S., de Vries, H., \& Hoving, C. (2012). Effectiveness of a web-based multiple tailored smoking cessation program: A randomized controlled trial among Dutch adult smokers. Journal of Medical Internet Research, 14(3), e82.

Smit, E. S., Evers, S. M. A. A., de Vries, H., \& Hoving, C. (2013). Cost-effectiveness and cost-utility of Internetbased computer tailoring for smoking cessation. Journal of Medical Internet Research, 15(3), e57.

Sniehotta, F., Nagy, G., Scholz, U., \& Schwarzer, R. (2006). The role of action control in implementing intentions during the first weeks of behaviour change. British Journal of Social Psychology, 45(1), 87-106.

Sniehotta, F., Scholz, U., \& Schwarzer, R. (2006). Action plans and coping plans for physical exercise: A longitudinal intervention study in cardiac rehabilitation. British Journal of Health Psychology, 11(1), 2337.

Songur, A., Ozen, O. A., \& Sarsilmaz, M. (2010). The toxic effects of formaldehyde on the nervous system. In Songur, A., Ozen, O. A., \& Sarsilmaz, M. Reviews of Environmental Contamination and Toxicology (pp. 105-118). New York: Springer.

Springvloet, L., Lechner, L., Vries, H., Candel, M. J. J. M., \& Oenema, A. (2015). Short- and medium-term efficacy of a web-based computer-tailored nutrition education intervention for adults including cognitive and environmental feedback. Randomized Controlled Trial, 17(1), e23.

Sprott, D. E., Spangenberg, E. R., Block, L. G., Fitzsimons, G. J., Morwitz, V. G., \& Williams, P. (2006). The question-behavior effect: What we know and where we go from here. Social Influence, 1(2), 128-137.

Staff, R. T., Chapko, D., Hogan, M. J., \& Whalley, L. J. (2016). Life course socioeconomic status and the decline in information processing speed in late life. Social Science \& Medicine, 151, 130-138.

Stafford, T. F., Stafford, M. R., \& Schkade, L. L. (2004). Determining uses and gratifications for the Internet. Decision Sciences, 35(2), 259-288.

Stanczyk, N. E., Bolman, C., Muris, J. W. M., \& de Vries, H. (2011). Study protocol of a Dutch smoking cessation e-health program. BMC Public Health, 11(1), 847-847.

Stanczyk, N. E., Bolman, C., Smit, E. S., Candel, M. J. J. M., Muris, J. W. M., \& de Vries, H. (2014). How to encourage smokers to participate in web-based computer-tailored smoking cessation programs: A comparison of different recruitment strategies. Health Education Research, 29(1), 23-40. 
Stanczyk, N. E., Bolman, C., van Adrichem, M., Candel, M. J. J. M., Muris, J. W. M., \& de Vries, H. (2014). Comparison of text and video computer-tailored interventions for smoking cessation: Randomized controlled trial. Journal of Medical Internet Research, 16(3), e69.

Stanczyk, N. E., Smit, E. S., Schulz, D. N., de Vries, H., Bolman, C., Muris, J. W. M., \& Evers, S. M. A. A. (2014). An economic evaluation of a video- and text-based computer-tailored intervention for smoking cessation: A cost-effectiveness and cost-utility analysis of a randomized controlled trial. PLOS ONE, 9(10), e110117.

Statistics Netherlands. (2012). Home Internet access rate highest in the Netherlands. Retrieved from http://www.cbs.nl/en-GB/menu/themas/vrije-tijd-cultuur/publicaties/artikelen/archief/2012/20123636-wm.htm

Statistisches, \& Bundesamt. (2013). Retrieved from https://www.destatis.de/DE/ZahlenFakten/Gesellschaft Staat/BildungForschungKultur/Bildungsstand/Bildungsstand.html\#Tabellen

Stellefson, M., Hanik, B., Chaney, B., Chaney, D., Tennant, B., \& Chavarria, E. A. (2011). eHealth literacy among college students: A systematic review with implications for eHealth education. Journal of Medical Internet Research, 13(4), e102.

Strecher, V. J., McClure, J., Alexander, G., Chakraborty, B., Nair, V., Konkel, J., Greene, S., Couper, M., Carlier, C., \& Wiese, C. (2008). The role of engagement in a tailored web-based smoking cessation program: Randomized controlled trial. Journal of Medical Internet Research, 10(5), e36.

Sutton, S. (2008). How does the Health Action Process Approach (HAPA) bridge the intention-behavior gap? An examination of the model's causal structure. Applied Psychology: An International Review, 57(1), 66-74.

Swenberg, J. A., Moeller, B. C., Lu, K., Rager, J. E., Fry, R. C., \& Starr, T. B. (2013). Formaldehyde carcinogenicity research: 30 years and counting for mode of action, epidemiology, and cancer risk assessment. Toxicologic Pathology, 41(2), 181-189.

TailorBuilder. (2015). TailorBuilder. Retrieved from https://www.tailorbuilder.com/en/home.html

Talhout, R. (2012). Rapportage en regelgeving van tabaksingrediënten. Een vergelijking van Nederland met andere landen. Retrieved from http://rivm.openrepository.com/rivm/handle/10029/281054

te Poel, F., Bolman, C., Reubsaet, A., \& de Vries, H. (2009). Efficacy of a single computer-tailored e-mail for smoking cessation: Results after 6 months. Health Education Research, 24(6), 930-940.

Teh, J. L., King, D., Watson, B., \& Liu, S. (2014). Self-stigma, perceived stigma, and help-seeking communication in people with mental illness. PORTAL Journal of Multidisciplinary International Studies, 11(1), 1-18.

ter Huurne, E., \& Gutteling, J. (2008). Information needs and risk perception as predictors of risk information seeking. Journal of Risk Research, 11(7), 847-862.

Thompson, D., Bhatt, R., Vazquez, I., Cullen, K. W., Baranowski, J., Baranowski, T., \& Liu, Y. (2015). Creating action plans in a serious video game increases and maintains child fruit-vegetable intake: A randomized controlled trial. The International Journal of Behavioral Nutrition and Physical Activity, 12(1), 199.

Todkill, D., \& Powell, J. (2013). Participant experiences of an Internet-based intervention and randomised control trial: Interview study. BMC Public Health, 13, 1017.

Tyas, S. L., \& Pederson, L. L. (1998). Psychosocial factors related to adolescent smoking: A critical review of the literature. Tobacco Control, 7(4), 409-420.

Umberson, D., Crosnoe, R., \& Reczek, C. (2010). Social relationships and health behavior across life course. Annual Review of Sociology, 36, 139-157.

Umberson, D., \& Montez, J. K. (2010). Social relationships and health: A flashpoint for health policy. Journal of Health and Social Behavior, 51(1), S54-S66.

van 't Riet, J., Ruiter, R. A. C., Werrij, M. Q., \& de Vries, H. (2010). Investigating message-framing effects in the context of a tailored intervention promoting physical activity. Health Education Research, 25(2), 343354.

van den Brakel, M., \& Ament, P. (2010). Inkomensverschillen tussen en binnen gemeenten. Retrieved from http://www.cbs.nl/NR/rdonlyres/2A02251B-EEDC-4A20-98F9-6340AB03F596/0/2010k3v4p07art.pdf 
van der Wulp, N. Y., Hoving, C., \& de Vries, H. (2013). A qualitative investigation of alcohol use advice during pregnancy: Experiences of Dutch midwives, pregnant women and their partners. Midwifery, 29(11), e89e98.

van der Wulp, N. Y., Hoving, C., Eijmael, K., Candel, M. J. J. M., van Dalen, W., \& De Vries, H. (2014). Reducing alcohol use during pregnancy via health counseling by midwives and Internet-based computer-tailored feedback: A cluster randomized trial. Journal of Medical Internet Research, 16(12), e274.

van Deursen, A., \& Helsper, E. J. (2015). A nuanced understanding of Internet use and non-use among the elderly. European Journal of Communication, 30(2), 171-187.

van Deursen, A., \& van Dijk, J. (2009). Using the Internet: Skill related problems in users' online behavior. Interacting with Computers, 21(5-6), 393-402.

van Deursen, A., \& van Dijk, J. (2011). Internet skills and the digital divide. New Media \& Society, 13(6), 893911.

van Deursen, A., \& van Dijk, J. (2014). The digital divide shifts to differences in usage. New Media \& Society, 16(3), 507-526.

van Deursen, A., \& Van Dijk, J. (2015). Internet skill levels increase, but gaps widen: A longitudinal crosssectional analysis (2010-2013) among the Dutch population. Information, Communication and Society, 18(7), 782-797.

van Deursen, A., van Dijk, J., \& ten Klooster, P. (2015). Increasing inequalities in what we do online: A longitudinal cross sectional analysis of Internet activities among the Dutch population (2010 to 2013) over gender, age, education, and income. Telematics and Informatics, 32(2), 259-272.

van Dijk, J. (2005). The deepening divide: Inequality in the information society. Thousand Oaks, CA [etc.]: Sage Publications.

van Genugten, L., van Empelen, P., \& Oenema, A. (2014). Intervention use and action planning in a web-based computer-tailored weight management program for overweight adults: Randomized controlled trial. Journal of Medical Internet Research, 3(3), e31.

van Keulen, H. M., Bosmans, J. E., van Tulder, M. W., Severens, J. L., de Vries, H., Brug, J., \& Mesters, I. (2010). Cost-effectiveness of tailored print communication, telephone motivational interviewing, and a combination of the two: Results of an economic evaluation alongside the Vitalum randomized controlled trial. International Journal of Behavioral Nutrition and Physical Activity, 7(1), 64.

van Keulen, H. M., Mesters, I., Ausems, M., van Breukelen, G., Campbell, M., Resnicow, K., Brug, J., \& de Vries, H. (2011). Tailored print communication and telephone motivational interviewing are equally successful in improving multiple lifestyle behaviors in a randomized controlled trial. Annals of Behavioral Medicine, 41(1), 104-118.

van Osch, L., Beenackers, M., Reubsaet, A., Lechner, L., Candel, M., \& de Vries, H. (2009). Action planning as predictor of health protective and health risk behavior: An investigation of fruit and snack consumption. The International Journal of Behavioral Nutrition and Physical Activity, 6(69).

van Osch, L., Lechner, L., Reubsaet, A., \& de Vries, H. (2010). From theory to practice: An explorative study into the instrumentality and specificity of implementation intentions. Psychology and Health, 25(3), 351364.

van Osch, L., Reubsaet, A., Lechner, L., \& de Vries, H. (2008). The formation of specific action plans can enhance sun protection behavior in motivated parents. Preventive Medicine, 47(1), 127-132.

van Stralen, M. M., de Vries, H., Mudde, A. N., Bolman, C., \& Lechner, L. (2011). The long-term efficacy of two computer-tailored physical activity interventions for older adults: Main effects and mediators. Health Psychology: Official Journal of the Division of Health Psychology, American Psychological Association, 30(4), 442-452.

Vandelanotte, C., Reeves, M. M., Brug, J., \& De Bourdeaudhuij, I. (2008). A randomized trial of sequential and simultaneous multiple behavior change interventions for physical activity and fat intake. Preventive Medicine, 46(3), 232-237.

Venkatesh, V., Morris, M. G., Davis, G. B., \& Fred, D. (2003). User acceptance of information technology: Toward a unified view. MIS Quarterly, 27(3), 425-478. 
Verweij, A. (2008). Onderwijsdeelname: Indeling opleidingsniveau. Retrieved from http://www.nationaalkompas.nl/bevolking/scholing-en-opleiding/indeling-opleidingsniveau/

Vlismas, K., Stavrinos, V., \& Panagiotakos, D. B. (2009). Socio-economic status, dietary habits and healthrelated outcomes in various parts of the world: A review. Central European Journal of Public Health, 17(2), 55-63.

Voncken-Brewster, V., Tange, H., de Vries, H., Nagykaldi, Z., Winkens, B., \& van de Weijden, T. (2015). A randomized controlled trial evaluating the effectiveness of a web-based, computer-tailored selfmanagement intervention for people with or at risk for COPD. International Journal of Chronic Obstructive Pulmonary Disease, 10(1), 1061-1073.

Voncken-Brewster, V., Tange, H., de Vries, H., Nagykaldi, Z., Winkens, B., \& van der Weijden, T. (2013). A randomised controlled trial testing a web-based, computer-tailored self-management intervention for people with or at risk for chronic obstructive pulmonary disease: A study protocol. BMC Public Health, 13(1), 557.

Vroege, D. P., Wijsman, C. A., Broekhuizen, K., de Craen, A. J. M., van Heemst, D., van der Ouderaa, F. J. G., van Mechelen, W., Slagboom, P. E., Catt, M., Westendorp, R. G. J., Verhagen, E. A. L. M., \& Mooijaart, S. P. (2014). Dose-response effects of a web-based physical activity program on body composition and metabolic health in inactive older adults: Additional analyses of a randomized controlled trial. Journal of Medical Internet Research, 16(12), e265.

Wallston, K. A. (1992). Hocus-pocus, the focus isn't strictly on locus: Rotter's social learning theory modified for health. Cognitive Therapy and Research, 16(2), 183-199.

Walthouwer, M. J., Oenema, A., Lechner, L., \& de Vries, H. (2015). Comparing a video and text version of a web-based computer-tailored intervention for obesity prevention: A randomized controlled trial. Journal of Medical Internet Research, 17(10), e236.

Walthouwer, M. J., Oenema, A., Soetens, K., Lechner, L., \& de Vries, H. (2013). Systematic development of a text-driven and a video-driven web-based computer-tailored obesity prevention intervention. BMC Public Health, 13(1), 978.

Walthouwer, M. J., Oenema, A., Soetens, K., Lechner, L., \& de Vries, H. (2014). Are clusters of dietary patterns and cluster membership stable over time? Results of a longitudinal cluster analysis study. Appetite, 82(0), 154-159.

Wangberg, S. C., Andreassen, H. K., Prokosch, H.-U., Santana, S. M. V., Sørensen, T., \& Chronaki, C. E. (2008). Relations between Internet use, socio-economic status (SES), social support and subjective health. Health Promotion International, 23(1), 70-77.

Wantland, D. J., Portillo, C. J., Holzemer, W. L., Slaughter, R., \& McGhee, E. M. (2004). The effectiveness of web-based vs. non-web-based interventions: A meta-analysis of behavioral change outcomes. Journal of Medical Internet Research, 6(4), e40.

Ward, M. M., Guthrie, L. C., \& Butler, S. C. (2009). Time perspective and socioeconomic status: A link to socioeconomic disparities in health? Social Science \& Medicine (1982), 68(12), 2145-2151.

Ware, J. E., Kosinski, M., \& Keller, S. D. (1996). A 12-item short-form health survey: Construction of scales and preliminary tests of reliability and validity. Medical Care, 34(3), 220-233.

Ware, J. E., Kosinski, M., \& Keller, S. D. (1998). SF-12: how to score the SF-12 physical and mental health summary scales QualityMetric Inc. Lincoln, RI.

Wasserman, I. M., \& Richmond-Abbott, M. (2005). Gender and the Internet: Causes of variation in access, level, and scope of use. Social Science Quarterly, 86(1), 252-270.

Watkins, I., \& Xie, B. (2014). eHealth literacy interventions for older adults: A systematic review of the literature. Journal of Medical Internet Research, 16(11), e225.

Webb, T., Joseph, J., Yardley, L., \& Michie, S. (2010). Using the Internet to promote health behavior change: A systematic review and meta-analysis of the impact of theoretical basis, use of behavior change techniques, and mode of delivery on efficacy. Journal of Medical Internet Research, 12(1), e4.

Webster, J., Trevino, L. K., \& Ryan, L. (1994). The dimensionality and correlates of flow in human-computer interactions. Computers in Human Behavior, 9(4), 411-426. 
Wei, K. K., Teo, H. H., Chan, H. C., \& Tan, B. C. Y. (2011). Conceptualizing and testing a social cognitive model of the digital divide. Information Systems Research, 22(1), 170-187.

Weinkauf, M. (2012). Benjamini-Hochberg. In BenjaminiHochberg.xIsx (Ed.).

Weinstein, N. D., \& Nicolich, M. (1993). Correct and incorrect interpretations of correlations between risk perceptions and risk behaviors. Health Psychology, 12(3), 235-245.

Weiser, E. B. (2000). Gender differences in Internet use patterns and Internet application preferences: A twosample comparison. Cyberpsychology \& Behavior, 3(2), 167-178.

Wendel-Vos, G. C., Schuit, A. J., Saris, W. H. M., \& Kromhout, D. (2003). Reproducibility and relative validity of the short questionnaire to assess health-enhancing physical activity. Journal of Clinical Epidemiology, 56(12), 1163-1169.

Weyers, S. (2008). Soziale Ungleichheit, soziale Beziehungen und Gesundheitsverhalten. In U. Bauer, U. H. Bittlingmayer, \& M. Richter (Eds.), Die Mehrdimensionalität der sozioökonomischen Lage -Konsequenzen für die Analyse gesundheitlicher Ungleichheit. Wiesbaden: Verlag für Sozialwissenschaften.

WHO. (2010). Global recommendations on physical activity for health (QT 255). Retrieved from http://apps.who.int/iris/bitstream/10665/44399/1/9789241599979_eng.pdf

WHO. (2012). Partial guidelines for implementation of Articles 9 and 10. World Health Organization. Retrieved from http://www.who.int/fctc/guidelines/Guideliness_Articles_9_10_rev_240613.pdf?ua=1

Wienert, J., \& Kuhlmann, T. (2015). A stitch in time saves nine: Things to consider when tailoring your online intervention. The European Health Psychologist, 17(1), 12-17.

Wild, K. V., Mattek, N. C., Maxwell, S. A., Dodge, H. H., Jimison, H. B., \& Kaye, J. A. (2012). Computer-related self-efficacy and anxiety in older adults with and without mild cognitive impairment. Alzheimer's \& Dementia, 8(6), 544-552.

Wilson, K., Senay, I., Durantini, M., Sánchez, F., Hennessy, M., Spring, B., \& Albarracín, D. (2015). When it comes to lifestyle recommendations, more is sometimes less: A meta-analysis of theoretical assumptions underlying the effectiveness of interventions promoting multiple behavior domain change. Psychological Bulletin, 141(2), 474-509.

Witte, K. (1994). Fear control and danger control: A test of the extended parallel process model (EPPM). Communications Monographs, 61(2), 113-134.

World Health Organization. (2013). Health literacy. The solid facts. Retrieved from http://www.euro.who.int/_data/assets/pdf_file/0008/190655/e96854.pdf

Zarcadoolas, C., Blanco, M., Boyer, J. F., \& Pleasant, A. (2002). Unweaving the Web: An exploratory study of low-literate adults' navigation skills on the World Wide Web. Journal of Health Communication, 7(4), 309-324.

Ziegelmann, J. P., Lippke, S., \& Schwarzer, R. (2006). Adoption and maintenance of physical activity: Planning interventions in young, middle-aged, and older adults. Psychology \& Health, 21(2), 145-163.

Zillien, N., \& Hargittai, E. (2009). Digital distinction: Status-specific types of Internet usage. Social Science Quarterly, 90(2), 274-291. 


\section{Valorization Addendum}

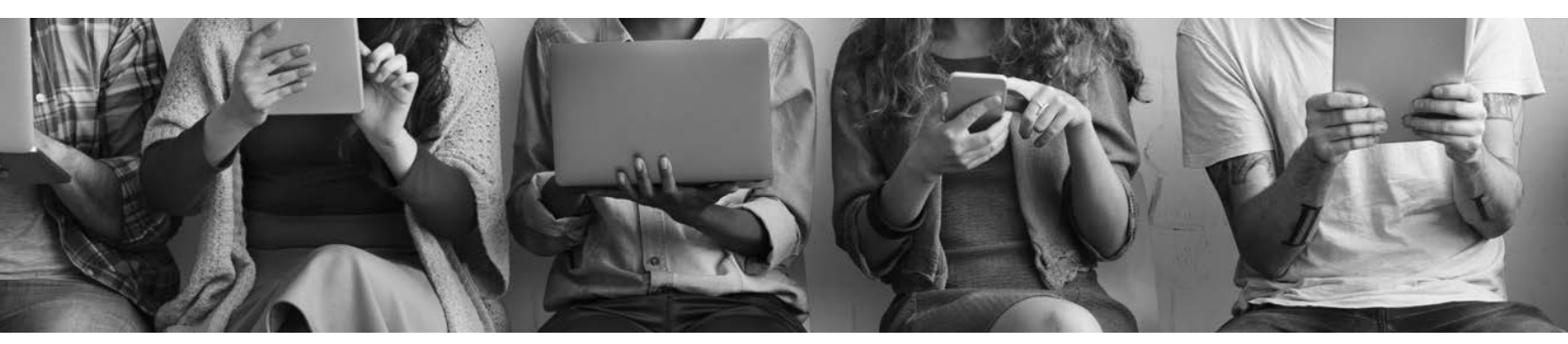


The present dissertation describes to what extend people with different personal characteristics use the Internet, health related websites, and Web-based computer tailored health interventions differently. This valorization addendum will discuss the findings in a broader perspective, beyond their scientific relevance.

\section{RELEVANCE}

Sociodemographic differences are not only measurable as inequalities of education, income, and occupational status (American Psychological Association \& Task Force on Socioeconomic Status, 2007), they also affect health. Health inequalities among different sociodemographic groups are responsible for a large part of the total burden of diseases in Europe (Dahlgren \& Whitehead, 2007; Mackenbach, et al., 2008). In the Netherlands, socioeconomic differences as well as differences between people with different personal characteristics (e.g., age, gender, and relationship) are also associated with variations in health and health behaviors. Hence, there is a need to reduce health inequalities among people with different characteristics, for example by providing health information and health interventions. The Internet is a promising channel to deliver health information and health interventions to improve health behavior. There are a multitude of websites presenting health related information and several Web-based computer tailored interventions have shown very promising results regarding changing health behavior and improving health outcomes (e.g., Broekhuizen, Kroeze, van Poppel, Oenema, \& Brug, 2012; Kohl, Crutzen, \& de Vries, 2013). Based on differences in health behavior among people with different personal characteristics, it could be assumed that there is also a difference in online use. If there are subgroups which might not use Web-based information to their full potential, it could increase the knowledge and health gap between people with different personal characteristics. If Web-based information and health interventions should have an impact, then such information and interventions must be used firstly. Whether the Internet and accordingly Web-based health related applications are used differently by people with different personal characteristics was assessed within this dissertation. Findings about differences between subgroups with regard to online use could provide indications how to adopt Web-based information and intervention to be useful to people with different sociodemographic characteristics.

\section{SCIENTIFIC RELEVANCE}

The findings of the present studies have shown that people with different personal characteristics use the Internet (Chapter 2), Web-based information (Chapter 3) and Web-based interventions (Chapter 4, 5) differently. These findings might give reasons to 
concern because those people who have in general an unhealthier lifestyle and those who have a higher risk to develop preventable diseases could potentially benefit the most from behavior change interventions. Also, the impact Web-based health related interventions could have might be biased by our finding that people with lower educational levels dropout more frequently from follow-up measurements (Chapter 6). While these studies have demonstrated that there are differences in online usage, the reasons for these inequalities are still unknown and further research might follow up on this in order to improve the usability of Web-based applications and to improve the measurable impact of interventions.

\section{SOCIAL RELEVANCE}

Inequalities in Internet use might result in disadvantages for specific groups that are not able to benefit from the information and health interventions provided online. Improving online use by tailoring the Web-based information to specific subgroups (like people with a lower level of education) might increase the usage and could reduce inequalities between these groups since it was found that those people less frequently search online for information, read news, use health interventions as recommended, and drop out earlier from Web-based health related studies. Adapting Web-based information and intervention might stimulate use of these applications and might reduce the health inequalities among subgroups with different personal characteristics. These implications might also be adaptable for other Web-based information and applications like online banking or searching for information and could improve accessing information and opportunities equally among subgroups with different personal characteristics.

\section{Economic relevance}

Inequalities in Internet use can have also implications for different economic parts. In particular, disadvantaged people (in terms of income, education, employment situations) often have an unhealthy lifestyle (smoking, high alcohol consumption, physical inactivity, unhealthy nutrition; (e.g., Charafeddine, Demarest, Van der Heyden, Tafforeau, \& Van Oyen, 2013; Gidlow, Johnston, Crone, Ellis, \& James, 2006; Pampel, Krueger, \& Denney, 2010)) and are more likely to suffer from preventable diseases such as obesity, diabetes, cancer, and cardio vascular diseases (Committee on Health and Behavior: Research Practice and Policy Board on Neuroscience and Behavioral Health, 2001; Lampert, Kroll, von der Lippe, Müters, \& Stolzenberg, 2013; Merletti, Galassi, \& Spadea, 2011). Therefore, it is important that it can be assured that those people at risk are able to make use of Web-based information and interventions to change their lifestyle. This could have a positive impact on reduced health care related costs (e.g., 
Drost, et al., 2016; Schulz, et al., 2014; Stanczyk, et al., 2014). Next to health related cost there are also other examples why improving Internet use skills could be beneficial for the economy. For example, unemployed people could be trained to seek for job advertisements online and could return to the job market earlier.

\section{Target groups}

The results have shown that people with different personal characteristics also use the Internet differently. It is not clear what causes these differences, but it is reasonable that there are specific skills necessary to use the Internet in the most beneficial way. Some people might be able to learn these skills by themselves or on the job, other people might have not these capabilities and possibilities. To reduce inequalities in online use in the future, it might be useful to implement Web-based learning materials in school so that children have to learn to use the Internet, find information, and being able to use it. By improving Internet literacy people might be able to use the Internet beneficially for different purposes inter alia to improve their health.

Furthermore, the findings form this dissertation can be useful for the government which often provides information to the general public. Using the Internet as medium to fulfil this task makes sense but as we have seen in Chapter 3, not many people are using the website to seek information in depth. Changing those websites to the needs of people with different personal characteristics (e.g., educational levels), should become a priority. 


\section{SUMMARY}

Today, in 2016, health inequalities exist between people who differ in personal characteristics such as education, income, occupation, or gender, even in prosperous countries such as the Netherlands. These health inequalities can be attributed to differences in health lifestyles such as lower levels of physical activity, poorer nutrition, and higher smoking prevalence, which can even result in shorter life expectancies among disadvantaged people. This provides reason to reduce health inequalities among people with different personal characteristics.

The Internet is a unique medium that can be used to distribute and quickly find information regarding nearly any topic worldwide at relatively low cost. The Internet has become an essential part of our everyday lives as we use it to gain information, to conduct online payments, to shop, and for health related purposes. In recent years, the quantity and quality of websites that inform about health related topics have increased enormously, as well as web-based health interventions aimed at changing health behaviors.

However, similarly to differences in health behavior, it can be assumed that there are also differences with regards to Internet use, health-related website use, and webbased health intervention use among people with different personal characteristics (i.e., age, gender, relationship, education, income, and occupation). This could be problematic because if people who would need such information and interventions in order to improve their health behaviors are not using them, then they might not receive the potential benefits. This could even increase the gap between people with different personal characteristics in terms of knowledge and health. In order to make web-based information and interventions equally useable, it is necessary to determine whether there are differences in use.

Therefore, the studies in this dissertation assessed differences in Internet use, health-related website use, and web-based health intervention use. Knowing whether and what differences there are between people with different personal characteristics with regards to online use allows us to adjust web-based information and applications to specific characteristics.

Chapter 1 provides the rationale for this dissertation and describes the background of health inequalities among people with different personal characteristics. Differences regarding Internet access and use are mentioned in relation to different personal characteristics, and related problems concerning differences in online use (i.e., the Internet, health-related website, and web-based health interventions) are mentioned. Furthermore, an overview of the research questions is given in this chapter.

Chapter 2 provides results from longitudinal data in six waves regarding seven different Internet activities (searching for information, reading news, being active on forums/blogs, 
emailing, visiting social networking websites, watching movies, and playing online games) from 2009 until 2014. The trends of Internet usage for different subgroups with regards to age, gender, educational level, level of income, and employment are discussed. This chapter reveals that all seven Internet activities increased in use over time, apart from being active on forums/blogs, which decreased instead. Information seeking is and was the most popular online activity. Activities regarding social interaction (emailing and using social networking websites), entertainment (gaming and movies), and activities for personal improvement (reading news) increased over time, but for all activities, differences between subgroups exist. In general, it was shown that some of the gaps between subgroups in terms of online use have even widened (e.g., gender, income and writing emails, and age and reading online news), while others did not narrow (e.g., educational level and searching for information or writing emails). These results show that people with lower educational levels and less income use the Internet more for entertainment purposes, while others use the Internet in order to gain personal benefits.

Chapter 3 describes the online behavior of participants in a randomized controlled trial of a website that provides information regarding tobacco additives. The goal of this study was to assess how visitors with different personal characteristics made use of the website in terms of the amount of information seeking and how visitors evaluated the website. Younger participants searched for more information on the website compared to older visitors and there was a positive relationship between information seeking and educational level. Furthermore, the website has been evaluated by females more positively compared to males and less positively by smokers. These results also indicate that website use differs among people with different personal characteristics, and those who would benefit from these health-related information (e.g., people with lower levels of education) made less use of it.

Chapter 4 presents the results of a study that investigated whether participants from the experimental arm of a web-based computer-tailored intervention aimed at improving five lifestyle behaviors (increasing fruit consumption, increasing vegetable consumption, increasing physical activity, reducing alcohol intake, and promoting smoking cessation) made use of the intervention as recommended. Participants were asked to participate in those specific online modules for which they did not meet the national guideline(s) for the respective behavior(s). Participants who started with fewer than the recommended number of modules in the intervention were defined as users who did not use the intervention as recommended. This study revealed that older participants, the unemployed, those within a relationship, females, and ill participants used the intervention more frequently as recommended compared to their counterparts. These results indicate that, in addition to Internet and websites, webbased health-related interventions are also used differently by people with different personal characteristics. 
Chapter 5 describes the results of a study that examined whether participants with different personal characteristics differ in their intervention use regarding the generation of action plans. In contrast to the other studies from this dissertation (see Chapters 2, 3, 4, and 6), these results showed fewer differences between personal characteristics and intervention use. Participants who were physically active at baseline were less likely to generate action plans concerning physical activity. With regards to the generation of action plans for fruit and vegetable consumption, participants with high risk perceptions and strong intentions to eat fruit and vegetables on a daily basis generated more action plans. Finally, the quality of action plans (in terms of specificity and instrumentality) was associated with having a relationship and low levels of negative outcome expectancies.

In Chapter 6, data from seven randomized controlled trials of web-based computertailored interventions were used in order to investigate dropout rates among participants with different educational levels, as well as participant evaluation of the interventions as a possible predictor of dropout attrition. In three studies, we found a higher study dropout attrition rate among participants with lower educational levels, whereas in two studies we found that middle educated participants had a higher dropout attrition rate compared to highly educated participants. In four studies, no such significant differences were found. Three of the seven studies showed that participants with lower or middle educational levels evaluated the interventions significantly better than highly educated participants. One study found lower intervention evaluations by lower educated participants compared to participants with middle educational levels. Low evaluation of the interventions was not a significant predictor of dropout at a later follow-up measurement in any of the studies. In summary, dropout attrition rates were either higher among participants with lower or middle educational levels compared with highly educated participants or the same, but never lower. Although lower educated participants evaluated the interventions better in approximately half of the studies, evaluation did not predict dropout attrition.

Chapter 7 presents a summary of the main findings of this dissertation. Possible explanations for the differences in online use, such as differences in health literacy skills and control beliefs, and the role of gratification are discussed. Methodological considerations are discussed as well as recommendations for future research. 


\section{Samenvatting}

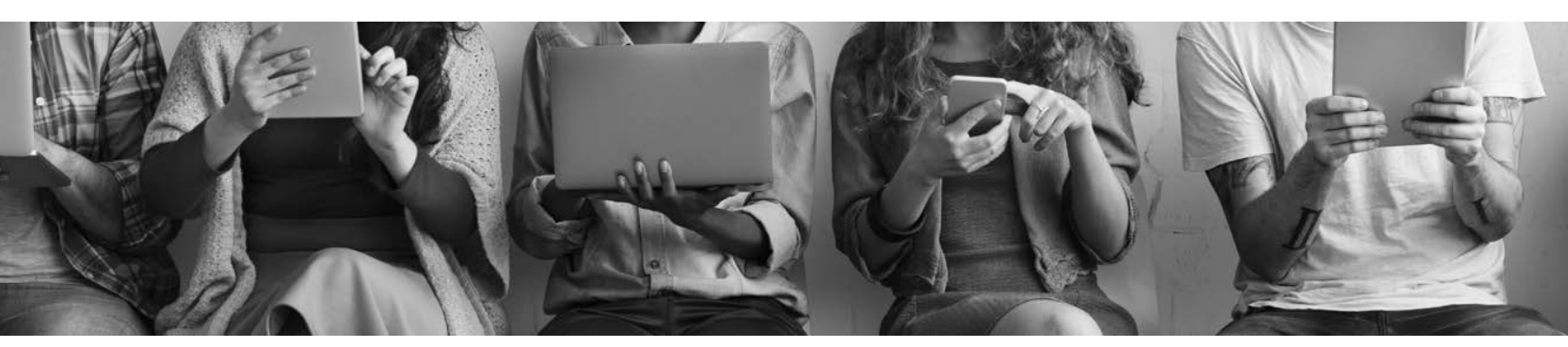




\section{SAMENVATTING}

Nog steeds, in 2016, zijn er gezondheidsverschillen tussen mensen met verschillende persoonskenmerken, zoals opleiding, inkomen, beroep of geslacht - ook in welvarende landen zoals Nederland. De gezondheidsverschillen kunnen worden toegeschreven aan verschillen in levensstijlen qua gezondheid zoals lagere niveaus van lichamelijke activiteit, slechtere voeding, en een hogere prevalentie van roken; verschillen die zelfs kunnen leiden tot een kortere levensverwachting onder de kansarmen. Dit geeft aanleiding om gezondheidsverschillen tussen groepen mensen met verschillende persoonskenmerken te verminderen.

Het internet is een uniek medium dat kan worden gebruikt voor het snel vinden en wereldwijd verspreiden van informatie over vrijwel elk onderwerp tegen relatief lage kosten. Internet is uitgegroeid tot een essentieel onderdeel in ons dagelijks leven als we het gebruiken om informatie te verkrijgen, om online betalingen uit te voeren, om te winkelen, maar ook voor de gezondheidsgerelateerde doeleinden. In de afgelopen jaren is de hoeveelheid en de kwaliteit van websites die informatie bieden over gezondheidsgerelateerde onderwerpen enorm toegenomen evenals web-based gezondheidsinterventies gericht op het bevorderen van gezond gedrag.

Net zoals bij verschillen in gezond gedrag, kan echter worden aangenomen dat er ook verschillen zijn met betrekking tot het gebruik van internet, gebruik van gezondheidsgerelateerde websites, en gebruik van web-based gezondheidsinterventies onder mensen met verschillende persoonskenmerken (in termen van leeftijd, geslacht, het hebben van een vaste relatie, opleiding, inkomen, beroep). Dit zou problematisch kunnen zijn, want als deze mensen dergelijke informatie en interventies missen om hun gedrag inzake gezondheid te verbeteren, dan kunnen ze de potentiële voordelen misschien niet ontvangen. Dit kan zelfs de kloof vergroten tussen mensen met verschillende persoonskenmerken inzake kennis en gezondheid. Om web-based informatie en interventies voor iedereen van nut te kunnen laten zijn, is het noodzakelijk om te bepalen of er verschillen zijn in gebruik.

Daarom gaat dit proefschrift over de verschillen in het gebruik van internet, gebruik van gezondheidsgerelateerd websites, en het gebruik van web-based gezondheidsinterventies. Het onderkennen van verschillen, en de aard van verschillen tussen mensen met verschillende persoonskenmerken met betrekking tot online gebruik, maakt het mogelijk om web-based informatie en applicaties aan die specifieke kenmerken aan te passen.

Hoofdstuk 1 presenteert de rationale voor dit proefschrift en beschrijft de achtergrond van de gezondheidsverschillen tussen mensen met verschillende persoonskenmerken. Verschillen inzake toegang tot internet en internetgebruik worden gerelateerd aan verschillende persoonskenmerken en aanverwante problemen met betrekking tot verschillen in online gebruik (dat wil zeggen, internet, gezondheidsgerelateerde 
website, web-based gezondheidsinterventies) worden genoemd. Verder wordt in dit hoofdstuk een overzicht gegeven van de onderzoeksvragen.

Hoofdstuk 2 presenteert de resultaten van longitudinale gegevens van de zes waves met betrekking tot zeven verschillende internetactiviteiten (informatie zoeken, het lezen van nieuws, actief zijn in forums / blogs, e-mailen, het bezoeken van sociale netwerkwebsites, films kijken, het spelen van online games) van 2009 tot 2014 . De trends van internetgebruik voor verschillende subgroepen met betrekking tot leeftijd, geslacht, opleidingsniveau, inkomen en werkgelegenheid worden besproken. Dit hoofdstuk laat zien dat alle zeven internetactiviteiten zijn toegenomen in gebruik, behalve die op gebied van bezoek aan forums / blogs; die zijn afgenomen. Het zoeken naar informatie is en was de meest populaire online activiteit. Activiteiten met betrekking tot sociale interactie (mailing, sociale netwerk websites), entertainment (games, films), en activiteiten voor persoonlijke groei (het lezen van nieuws) zijn toegenomen, maar er zijn verschillen tussen de subgroepen voor alle activiteiten. In het algemeen kan worden aangetoond dat een aantal lacunes zijn vergroot tussen subgroepen en online gebruik, (bijvoorbeeld qua geslacht, inkomen en het schrijven van e-mails, de leeftijd en het lezen van online nieuws), terwijl anderen verschillen niet minder geworden zijn (bijvoorbeeld, opleidingsniveau en het opzoeken van informatie of schrijven van emails). Deze resultaten laten zien dat mensen met een lager opleidingsniveau en minder inkomsten het internet eerder gebruiken voor entertainment-doeleinden, terwijl anderen internet gebruiken om persoonlijke voordelen te verwerven.

Hoofdstuk 3 beschrijft het online gedrag van de deelnemers aan een gerandomiseerde gecontroleerde studie op een website die informatie geeft over tabaksadditieven. Er is onderzocht hoe bezoekers met verschillende persoonskenmerken erken gebruik makkten van de website inzake de hoeveelheid informatievergaring en hoe bezoekers de website waardeerden. Jongere deelnemers zochten meer informatie op de website dan oudere bezoekers en er was een positief verband tussen zoeken naar informatie en het opleidingsniveau. Bovendien is de website door vrouwen positiever geëvalueerd in vergelijking met mannen en minder positief door rokers. Deze resultaten geven ook aan dat gebruik van de website verschilt tussen mensen met verschillende persoonskenmerken en dat degenen die zouden profiteren van deze gezondheidsgerelateerde informatie (bijvoorbeeld mensen met een lagere niveaus van onderwijs) er minder gebruik van maakten.

Hoofdstuk 4 presenteert de resultaten van een studie waarin onderzocht werd of de deelnemers uit de experimentele arm van een studie naar een web-based computer tailored interventie gericht op het verbeteren van vijf levensstijl gedragingen (het verhogen van de groente- en fruitconsumptie, verhoging van de lichamelijke activiteit, het verminderen van alcoholgebruik, en het bevorderen van het stoppen met roken) 
inderdaad gebruik gemaakt hebben van deze interventie zoals aanbevolen. Deelnemers werd gevraagd om deel te nemen aan die specifieke online modules waarvoor zij niet voldeden aan de nationale richtlijnen voor de respectievelijke gedragingen. Deelnemers die met minder dan het aanbevolen aantal modules van de interventie begonnen, werden gedefinieerd als gebruikers die de interventie niet hebben gebruikt zoals aanbevolen. Deze studie toonde aan dat de oudere deelnemers, werklozen, mensen binnen een vaste relatie, vrouwen, en zieke deelnemers de interventie vaker gebruikten, zoals aanbevolen in vergelijking met hun tegenhangers. Deze resultaten geven aan dat naast internet en websites ook web-based gezondheidsgerelateerde interventies anders werden gebruikt door mensen met verschillende persoonskenmerken.

Hoofdstuk 5 beschrijft de resultaten van een studie die onderzocht of de deelnemers met verschillende persoonskenmerken ook onderling verschillen in hun interventiegebruik met betrekking tot het starten van actieplannen. In tegenstelling tot de andere studies van dit proefschrift (in hoofdstuk 2, 3, 4 en 6) vertoonden deze resultaten minder verschillen tussen persoonskenmerken en het gebruik van de interventie. Deelnemers die lichamelijk actief waren bij aanvang, hadden minder kans om actieplannen met betrekking tot fysieke activiteit te genereren. Met betrekking tot opstarten van actieplannen voor de groente- en fruitconsumptie, maakten de deelnemers met een eigen hoge risicoperceptie en een sterke intentie om fruit en groenten te eten op een dagelijkse basis meer actieplannen. Tot slot wordt de kwaliteit van de actieplannen (in termen van specificiteit en instrumentaliteit) geassocieerd met het hebben van een relatie en lage niveaus van negatieve uitkomstverwachtingen.

In hoofdstuk 6 wordt een studie beschreven waarin data van zeven web-based adviesop-maat interventies zijn gebruikt om uitval onder de deelnemers met verschillende onderwijsniveaus alsook deelnemersevaluaties van de interventies als een mogelijke voorspeller van uitval te onderzoeken. In drie studies vonden we een hogere uitval onder de deelnemers met een lager opleidingsniveau, terwijl we in twee studies vonden, dat middelbaar opgeleide deelnemers een hogere uitval hadden in vergelijking met hoogopgeleide deelnemers. In vier studies werden dergelijke verschillen niet gevonden. Drie van zeven studies toonden aan dat deelnemers met een lagere of middelbare opleidingsniveau de interventies beduidend beter evalueerde dan hoogopgeleide deelnemers. Één studie wees op lagere evaluaties van interventie door lager opgeleiden deelnemers in vergelijking met de deelnemers met een middelste opleidingsniveau. Lage evaluatie van de maatregelen bleek geen belangrijke voorspeller van uitval in een nameting. Samengevat bleek dat de uitval hoger was onder de deelnemers met een lagere of middelbare opleidingsniveau in vergelijking met hoogopgeleide deelnemers of hetzelfde, maar nooit lager. Hoewel lager opgeleide deelnemers de interventies beter evalueerden in ongeveer de helft van de studies, had de evaluatie geen voorspellende waarde met betrekking tot uitval op nametingen. 
Hoofdstuk 7 geeft een overzicht van de belangrijkste bevindingen van dit proefschrift. Mogelijke verklaringen voor verschillen in online gebruik, zoals verschillen in gezondheidsgeletterdheid en de ervaren mate van controle, en de rol van behoeftebevrediging worden besproken. Methodologische overwegingen worden behandeld, alsmede aanbevelingen voor verder onderzoek. 



\section{Dankwoord}

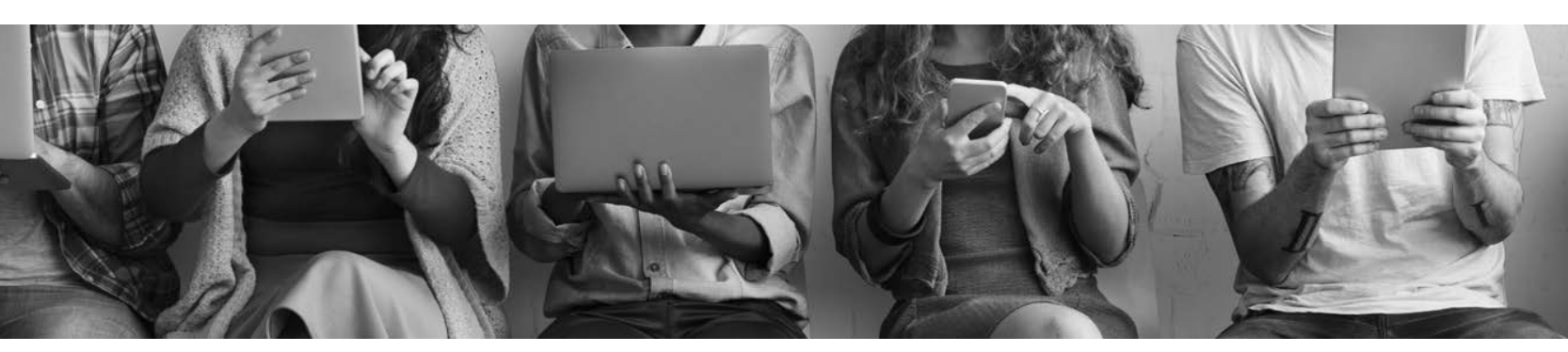


Bij het tot stand komen van dit proefschrift hebben een aantal mensen een belangrijke bijdrage geleverd en ik wil graag de tijd nemen om hen dank te zeggen.

Een speciaal woord van dank wil ik uitspreken aan mijn promotieteam. Graag wil ik beginnen met dank te zeggen aan Hein, mijn promotor. Hein, je bent een hele bijzondere man en ik kan mij nog goed herinneren hoe ik je voor het eerst in een college in actie heb gezien. Je straalde zoveel energie uit en wist ons studenten direct enthousiast te maken over het onderwerp. Deze karaktereigenschap bewonder ik nog steeds aan jou. Door je onvermoeibare passie, verbeeldingskracht en het vermogen om in kleine ideeën een groot potentieel te zien, wist je mij te inspireren om het beste uit mijn onderzoek te halen. Ik kon altijd op jou rekenen. Bedankt voor de vrijheid die je mij gaf om me als onderzoeker te ontwikkelen en bedankt voor je vertrouwen in mijn kwaliteiten.

Een tweede woord van groot dank gaat uit naar Rik: Rik, je bent altijd enthousiast, in een goed humeur en bereid om te helpen. Als co-promotor en dagelijks begeleider stond jouw deur altijd voor me open. Ik ken niemand die zo snel op e-mails reageert en bij elke moeilijke vraag wel een antwoord kent. Ik ben erg blij dat ik zo veel van jou mocht leren! Dank hiervoor en ook voor je vertrouwen in mij.

I would like to thank Sonia for giving me the chance to work on the RENATA-project. Even if we were geographically separated, I could always reach you and you made me feel welcome in your team. I will always remember your seminar about work-life balance which helped me to find a good balance during my PhD-period - and I hope I will remember these words until my retirement.

Tim, Julian, and Vera: I was happy to work with you together on our RENATAproject. Thank you all for your input and feedback on the articles we wrote together.

Verder mocht ik met een aantal andere co-auteurs samenwerken die een belangrijke bijdrage geleverd hebben aan de verschillende hoofdstukken uit deze proefschrift: Math Candel, Iman Elfeddali, Ciska Hoving, Stef Kremers, Anne Kienhuis, Reinskje Talhout, Francine Schneider, Daniela Schulz, Eline Smit, Nicola Stanczyk, Huibert Tange, Michel Walthouwer en Viola Voncken-Brewster. Hartelijk bedankt voor de leerzame samenwerking, jullie kennis, tijd en constructieve commentaren.

Graag wil ik ook mijn dank uitspreken aan alle lieve GVO collega's uit Maastricht. Wat is het toch een gezellige groep (en een zo'n grote groep, waardoor ik niet iedereen hier bij naam kan noemen). Bedankt voor alle leuke uitjes, borrels, saladelunches, congresbezoeken, etentjes, inhoudelijke discussies en helpende handjes. Ondanks dat iedereen het druk heeft, was er altijd tijd voor een gesprek. Als jullie geen nuttige tips hadden dan was er wel tijd voor flauwekul. Aan sommige grappen denk ik nog steeds heel graag met een glimlach terug. Bedankt hiervoor :-) Ik zal deze leuke sfeer zeker missen!

Auch meinen Paranymphen Kristina und Karin möchte ich ganz herzlich Danke sagen! Ihr habt von Anfang bis Ende mein Projekt mitbekommen und kennt es 
wahrscheinlich auch in- und auswendig. Schön zu wissen, dass ihr während der Verteidigung hinter mir steht.

Liebe Freunde nah und fern, liebe Stammtisch-Mädchen, danke für die schönen Momente während und nach der Arbeit. Ich freue mich immer, Zeit mit euch zu verbringen und in den letzten Jahren habe ich die Zeit mit euch genutzt, um Motivation zu schöpfen, dieses Büchlein hier fertig zu bekommen. Das feiern wir bald ganz groß!

Liebe Familie, danke dafür, dass ich mich immer auf euch verlassen kann! Seit dem Studium fragt ihr euch was ich eigentlich den ganzen Tag mache. Vielleicht wisst ihr mehr nachdem ihr dieses Buch gelesen habt. Wahrscheinlich habt ihr darauf aber wenig Lust. So oder so, ab jetzt werde ich wieder mehr Zeit für euch haben. Auf jeden Fall danke ich euch sehr für eure Unterstützung in den vergangenen Jahren!

Herzallerliebster Tommy, es ist wunderbar, dass du an meiner Seite bist. Danke für alles! 

Dominique Alexandra Reinwand was born on February, $5^{\text {th }}, 1984$ in Oberwesel, Germany. In 2004, she received her Gymnasium Diploma at the "Kreuzau Gymnasium" in Germany. Afterwards, she moved to Maastricht to study Public Health at Maastricht University from where she graduated in 2008 with her bachelor diploma. She took one year off

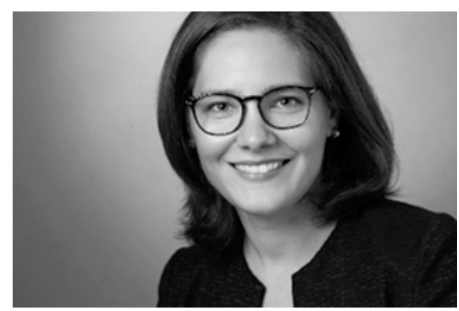
University to follow internships at the "Fachgesellschaft für Ernährung und Prävention e.V." and at the department of health education and promotion at the local authority in Aachen, Germany.

In 2009 Dominique started with the master Health Education and Promotion at Maastricht University. In 2011 she started working at the Institute on Addiction and Prevention Research of the Nordrhein-Westfalen Catholic University of Applied Sciences, Germany. In 2012 she received a scholarship from the German "Wilhelmstiftung für Rehabilitationsforschung" to conduct her PhD project at the Jacobs Center on Lifelong Learning and Institutional Development (JCLL) of the Jacobs University GgmbH, Bremen and the School for Public Health and Primary Care (CAPHRI), Department of Health Promotion at the Faculty of Health, Medicine and Life Science of Maastricht University. The focus on her PhD-project was the use of Web-based interventions among different sociodemographic subgroups.

As of March 2016, Dominique is working as postdoctoral researcher at the University of Cologne, Faculty of Human Science, Department of Rehabilitation Science and Gerontology. 
Publication List

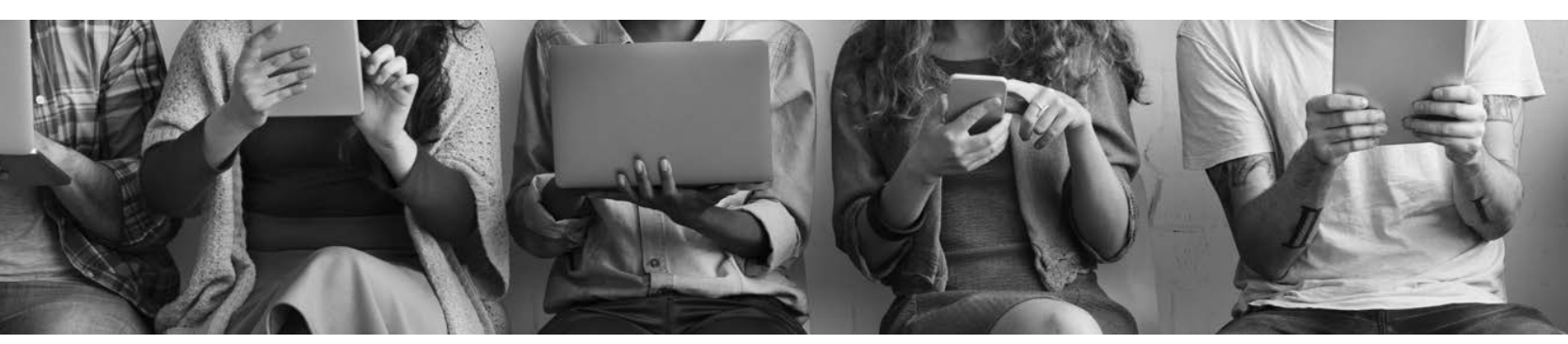





\section{D.D. $25^{\text {TH }}$ DECEMBER 2016 \\ PUBLICATIONS FROM THIS DISSERTATION}

Reinwand, D. A., Crutzen, R., Storm, V., Wienert, J., Kuhlmann, T., de Vries, H. \& Lippke, S. (2016). Generating and predicting high quality action plans to facilitate physical activity and fruit and vegetable consumption: Results from an experimental arm of a randomised controlled trial. BMC Public Health, 16(1), 317.

Reinwand, D. A., Schulz, D. N., Crutzen, R., Kremers, S. P. J. \& de Vries, H. (2015). Who follows eHealth interventions as recommended? A study of participants' personal characteristics from the experimental arm of a randomized controlled trial. Journal of Medical Internet Research, 17(5), e115.

Reinwand D. A., Crutzen, R., Elfeddali, I., Schneider, F., Schulz, D. N., Smit, E. S., Stanczyk, N. E., Tange, H., Voncken-Brewster, V., Walthouwer, M. J. L., Hoving, C. \& de Vries, H. (2015). Impact of educational level on study attrition and evaluation of webbased computer-tailored interventions: Results from seven randomized controlled trials. Journal of Medical Internet Research, 17(10), e228

Reinwand, D. A., Candel, M. J. J. M., de Vries, H. \& Crutzen, R. (submitted). Personal characteristics and trends across time of Internet use in the Netherlands: A multiwave panel study.

Reinwand, D. A., Crutzen, R., Kienhuis, A., Talhout, A. \& de Vries, H. (submitted). Website use and effects of online information about tobacco additives. A randomized controlled trial.

\section{OTHER PUBLICATIONS}

Storm, V., Reinwand, D. A., Wienert, J., Kuhlmann, T., de Vries, H. \& Lippke, S. (2016). Brief report: Compensatory health beliefs are negatively associated with intentions for regular fruit and vegetable consumption when self-efficacy is low. Journal of Health Psychology

Storm V., Dörenkämper J., Reinwand D. A., Wienert, J., de Vries, H. \& Lippke, S. (2016). Effectiveness of a web-Based computer-tailored multiple-lifestyle intervention for people interested in reducing their cardiovascular risk: A randomized controlled trial. Journal of Medical Internet Research, 18(4), e78

Reinwand, D. A., Kuhlmann, T., Wienert, J., de Vries, H. \& Lippke, S. (2013). Designing a theory- and evidence-based tailored eHealth rehabilitation aftercare program in Germany and the Netherlands: Study protocol, BMC Public Health, 13: 1081 
Schulz, D. N., Candel, M. J. J. M., Kremers, S. P., Reinwand, D. A., Jander, A. \& de Vries, H. (2013). Effects of a web-based tailored intervention to reduce alcohol consumption in adults: Randomized controlled trial. Journal of Medical Internet Research, 15(9), e206.

Storm, V., Reinwand, D. A., Wienert, J. \& Lippke, S. (submitted). Quality of life and stages of behavioural change for physical activity in people motivated to reduce their cardiovascular risk.

\section{REPORT}

Reinwand, D. A., Crutzen, R. \& de Vries, H. (2015). Evaluatieonderzoek over de website www.tabakinfo.nl. Onderzoek naar de impact van gezondheidsvoorlichting over het onderwerp tabaksadditieven onder het Nederlandse publiek. Rapport voor het RIVM 


\section{Abbreviations}

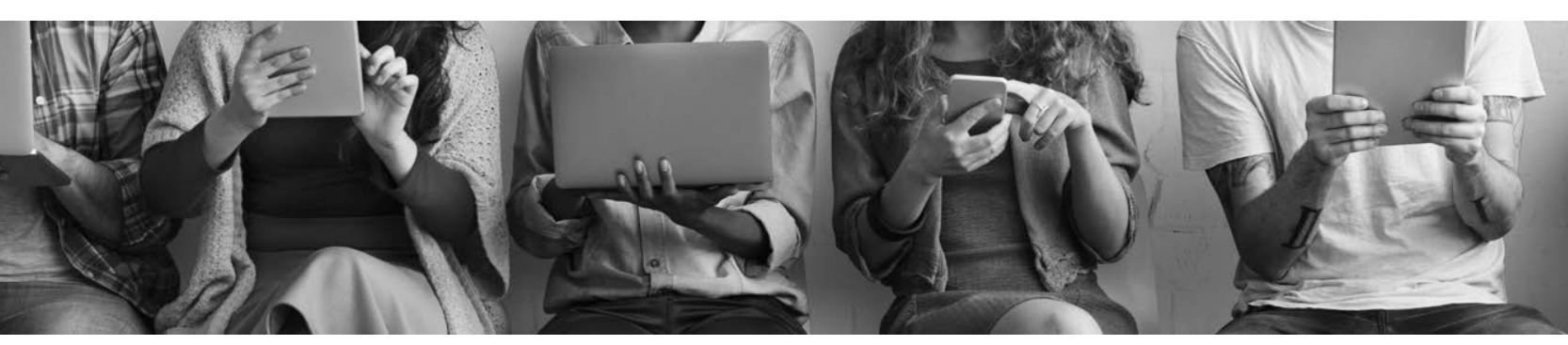



AEL Alcohol-Everything within the Limit

AP Action plan

COPD Chronic obstructive pulmonary disease

$\mathrm{Cl} \quad$ Confidence Interval

CT Computer-tailoring

DF Degrees of freedom

ES Effect size

FFQ Food Frequency Questionnaire

FV Fruit and vegetable

HAPA Health Action Process Approach

HSD Honestly significant difference

LISS Longitudinal Internet Studies for the Social sciences

MESS Measurement and Experimentation in the Social Sciences

MHB My Healthy Behavior

MYB Master Your Breath

OR Odds ratio

PA Physical activity

PAS Personal Advice in Stopping smoking

QFV Quantity-Frequency-Variability

RCT Randomized controlled trial

RIVM National Institute for Public Health and the Environment (Rijksinstituut voor Volksgezondheid en Milieu)

RHA Regional Health Authorities

SD Standard deviation

SES Socio-economic status

SF-12 Short Form Health Survey questionnaire

SQ4U Stay Quit for You

SQUASH Short Questionnaire to Assess Health-Enhancing Physical Activity

STQ Support to Quit

TA Tobacco additives

WIB Weight in Balance 


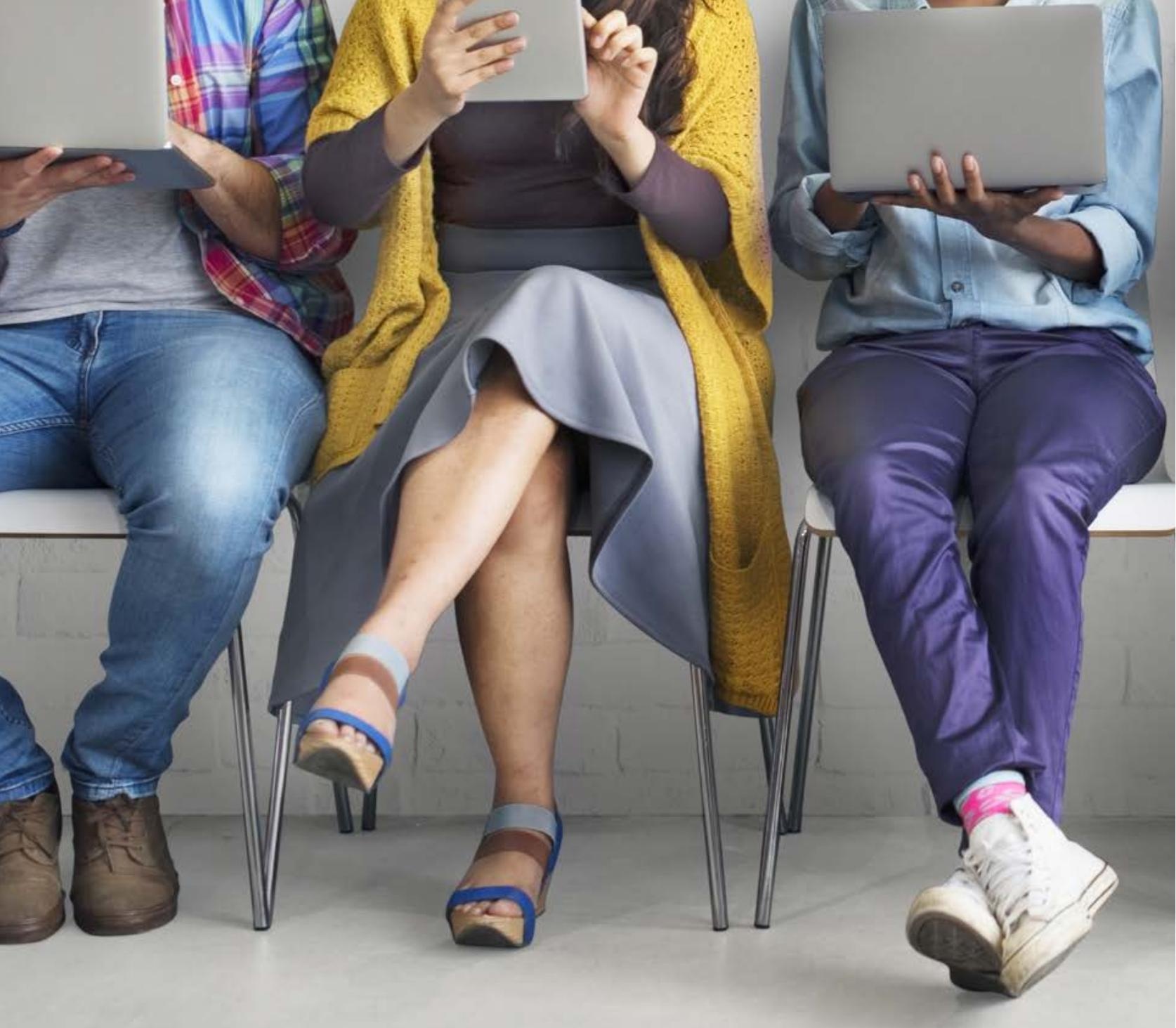

